Fabrication of Photonic Crystals and Nanocavities 
Promotiecommissie

Promotor Prof. dr. W. L. Vos

Assistent Promotor Dr. R. W. Tjerkstra

Voorzitter Prof. dr. M. J. Peters

Overige leden Prof. dr. M. C. Elwenspoek

Prof. dr. U. Gösele

Prof. dr. A. Lagendijk

Dr. I. D. Setija

Prof. dr. E. Vlieg

$\begin{array}{ll}\text { Paranimfen } & \text { B. H. Husken }\end{array}$

L. G. Woldering

The work in this thesis was supported by 'NanoNed', a nanotechnology program of the Dutch Ministry of Economic Affairs, and this work is part of the research program of the 'Stichting voor Fundamenteel Onderzoek der Materie (FOM)', which is financially supported by the 'Nederlandse Organisatie voor Wetenschappelijk Onderzoek (NWO)'.

This work was carried out at the

Complex Photonic Systems Group, Department of Science and Technology and MESA ${ }^{+}$Institute for Nanotechnology, University of Twente, P.O. Box 217, 7500 AE Enschede, The Netherlands. where a limited number of copies of this thesis is available.

Cover: impression of an inverse woodpile crystal illuminated by a light source inside a milled sphere (rendered by PovRay v3.6).

This thesis can be downloaded from www.photonicbandgaps.com.

Printed by Gildeprint Drukkerijen B.V., Enschede, The Netherlands (2008). ISBN: 978-90-365-2705-7 


\title{
Fabrication of Photonic Crystals and Nanocavities
}

\section{PROEFSCHRIFT}

ter verkrijging van

de graad van doctor aan de Universiteit Twente, op gezag van de rector magnificus, prof. dr. W.H.M. Zijm, volgens besluit van het College voor Promoties in het openbaar te verdedigen op vrijdag 5 september 2008 om 13.15 uur

\author{
door
}

Léon Alexis Woldering

geboren op 30 oktober 1974

te Enschede 
Dit proefschrift is goedgekeurd door:

prof. dr. W. L. Vos en dr. R. W. Tjerkstra 


Voor Cas en Eline. 



\section{Contents of this Thesis}

1 Introduction 13

1.1 Controlling and using light . . . . . . . . . . . 13

1.2 Photonic crystals . . . . . . . . . . . . . . 15

1.3 Fabrication of three-dimensional photonic crystals . . . . . 19

1.3 .1 Inverse opals . . . . . . . . . . . . . . . . . 19

1.3.2 Simple-cubic photonic crystals . . . . . . . . . 20

1.3.3 Diamond-like photonic crystals . . . . . . . . . . 20

1.3.4 Inverse woodpile photonic crystals . . . . . . . . . 22

1.4 Optical cavities in a three-dimensional photonic crystal . . . 24

1.5 Applications of photonic crystals . . . . . . . . . 26

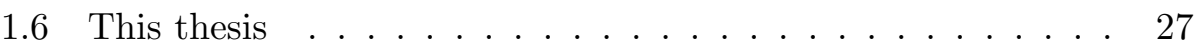

2 Fabrication and optical properties of inverse woodpile photonic crystals

2.1 Introduction . . . . . . . . . . . . . . . . . 29

2.2 Geometry of the cubic inverse woodpile structures . . . . . 30

2.3 Fabrication scheme . . . . . . . . . . . . . 31

2.3.1 Photo electrochemical etching . . . . . . . . . . . 34

2.3.2 Reactive ion etching . . . . . . . . . . . . . 34

2.3.3 The holder wafer and mounting of tilted sample . . . 36

2.4 Deviations from a perfect cubic inverse woodpile crystal . . . 37

2.4.1 Radius $R$ to lattice parameter $a$ different from $0.24 \quad$. 38 
2.4.2 A $\Delta y$ displacement between the two sets of pores . . . 41

2.4.3 Angular misalignments . . . . . . . . . . . . . . . 41

2.4.4 Tapered pores . . . . . . . . . . . . . . 45

2.4.5 Different cylinder radii of the two sets of pores . . . . 49

2.5 Optimized broad photonic band gap . . . . . . . . . 52

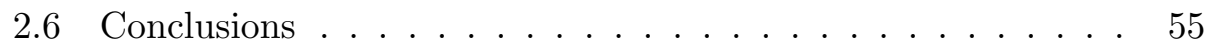

3 Photo electrochemical etching of pores in silicon 57

3.1 Introduction . . . . . . . . . . . . 57

3.1.1 Photo electrochemical etching . . . . . . . . . 57

3.1 .2 Etching parameters . . . . . . . . . . . . 59

3.1 .3 Chemical reactions . . . . . . . . . . . . . . . 62

3.2 Etching setup . . . . . . . . . . . . 63

3.3 Experimental methods $\ldots \ldots \ldots \ldots 66$

3.4 Fabrication of pore tips $\ldots \ldots \ldots \ldots$

3.5 Etched pores . . . . . . . . . . . . . . . . . . . . 74

3.6 Discussion . . . . . . . . . . . . . . . . . . 77

Step i: measure $I$ - $U_{\text {elec }}$ curves . . . . . . . . . . . . . 79

Step ii: calculate $I-U_{\text {wafer }}$ curves . . . . . . . . . . 81

Step iii: determine $U_{\text {wafer }}$ of the etching experiments 82

Step iv: analysis of the etching results . . . . . . . . . 82

3.7 Stability and reproducibility . . . . . . . . . . . 85

3.8 Conclusions . . . . . . . . . . . . . . . . 88

4 Reactive ion etching of deep nanopores $\quad 89$

4.1 Introduction . . . . . . . . . . . . . 89

4.1.1 Fabricating deep nanopores . . . . . . . . . . 89

4.1 .2 Reactive ion etching . . . . . . . . . . . . . . 91

4.2 Experimental methods . . . . . . . . . . . . . . . 94

4.3 Results and discussion . . . . . . . . . . . . . . . . . . . 97

4.3.1 Deep UV Lithography . . . . . . . . . . . . . . 97

4.3.2 Nanopores in silicon . . . . . . . . . . . . . . 99

4.3.3 Exploring seven parameters of the etching process . . 102

i) Total etch duration . . . . . . . . . . . . . . 102

ii) Etchant flow . . . . . . . . . . . . . . . . . . . . . 102

iii) Flow in the protection step . . . . . . . . . . . . . . 104

iv) Duration of the protection step . . . . . . . . 106

v) Influence of the inductively coupled plasma source . 108

vi) Capacitively coupled plasma source . . . . . . . . . . 108

vii) Distance between substrate and source . . . . . . 111 
4.3.4 Mask geometry . . . . . . . . . . . . . . 113

Mask aperture diameter . . . . . . . . . . . . 113

Influence of the pattern density on the sidewall erosion 116

4.4 Theoretical analysis of time dependent pore depth . . . . . 117

4.5 Ultimate etching . . . . . . . . . . . . . . . . . . . . 122

4.5.1 Centered rectangular lattices . . . . . . . . . . 122

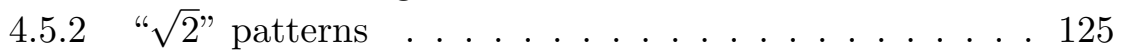

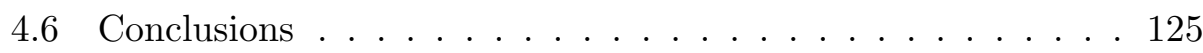

4.7 Appendix A . . . . . . . . . . . . . . . . 128

5 Fabrication and testing of the holder wafer 129

5.1 Introduction . . . . . . . . . . . . . . . 129

5.2 Fabrication of the holder wafer . . . . . . . . . . . 132

5.3 Preparation of the cross-section surface of an etched sample 134

5.4 Alignment of samples in the holder wafer . . . . . . . . 139

5.5 Mask fabrication on samples mounted in a holder wafer . . . 141

5.6 Conclusions . . . . . . . . . . . . . . . . . . . . . 142

6 Focused ion beam milling of nanocavities in artificial opals $\quad \mathbf{1 4 5}$

6.1 Introduction . . . . . . . . . . . . . . . 145

6.1.1 Optical cavities in photonic crystals . . . . . . . . 145

6.1.2 Embedding a cavity in 3-dimensional artificial opals . 146

6.2 Experimental methods . . . . . . . . . . . . . . 148

6.3 Milling results . . . . . . . . . . . . . . . 150

6.3.1 Milled defects and arrays of defects . . . . . . 150

6.3.2 Controlling the cavity profile . . . . . . . . 155

6.3.3 Milling with too high currents . . . . . . . . . . 157

6.4 Discussion . . . . . . . . . . . . . . . . . . 159

6.5 Scattering of light by nano-cavities . . . . . . . . . . 160

6.6 Conclusions . . . . . . . . . . . . . . . . . . 164

7 Reflectivity measured on fabricated structures 165

7.1 Introduction . . . . . . . . . . . . . 165

7.2 Optical setup . . . . . . . . . . . . . 165

7.3 Optical reflectivity of two-dimensional photonic crystals . . . 167

7.4 Focused ion beam milling of prototype three dimensional photonic crystals . . . . . . . . . . . . . . 174

7.4.1 Monoclinic photonic structures . . . . . . . . . . . . 174

7.4.2 Inverse woodpile photonic crystal . . . . . . . . 176 
7.5 Reflectivity of a three-dimensional inverse woodpile photonic

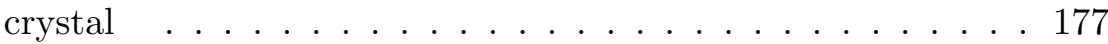

7.6 Conclusions . . . . . . . . . . . . . . . . . 180

7.7 Appendix A . . . . . . . . . . . . . . . . 182

8 Summary and outlook 183

$\begin{array}{ll}\text { References } & 187\end{array}$

$\begin{array}{ll}\text { Nederlandse samenvatting } & 205\end{array}$

$\begin{array}{ll}\text { Dankwoord } & 215\end{array}$ 


\section{Introduction}

\subsection{Controlling and using light}

It is undisputable that light is essential for life. Everything we eat, giving us energy and health, grows because of light from the sun. In everyday life, light is in many ways crucial to observe, learn, and act. For instance, light allows us to know where we are after sunset: by looking at light from the star "Polaris" one knows where north is [1], light from a lighthouse assists sailors to reach their destinations, and, inland, light from street lanterns allows us to find our way.

The importance of light is reflected in the efforts by civilizations to use, control, and create light. Quite early in history, mankind started to fabricate devices to use light more efficiently. For example, mirrors allowed light of objects to be reflected. The oldest manufactured mirrors have been made approximately 8000 years ago. They have been found in graves of women in Anatolia, south central modern day Turkey. They were made from obsidian, highly polished, and had a convex surface, thus requiring less material. Other early mirrors found across the world were flat, convex, and also concave to allow magnification [2]. Lenses were constructed to magnify objects, or simply to harvest and focus sunlight to start a fire. Examples of early fabricated lenses have been found in Knossos, Crete [3]. These dated from as early as 1400 B.C. In Nimrud, modern day Iraq, another lens was found that is over three thousand years old. What precisely the Assyrians used this object for is apparently disputed, but possible uses include astronomy and intricate crafting.

A few centuries ago, lenses were used to build the first microscopes and telescopes. The first microscopes were made around 1600 by placing two lenses in a tube. The invention of the microscope is attributed to Dutch 
spectacle makers Hans Janssen, his son Zacharias Janssen, and Hans Lippershey [4]. In 1608 Hans Lippershey also made the first telescope [5]. After their invention, telescopes were mainly used for military purposes. Galileo was the first to point a telescope towards stars and planets, of which he published the first results in 1610. With telescopes and microscopes, light could now be used to look at objects far away and to look at very small objects.

Methods and apparatuses were sought to generate light. Examples include the burning of wood in a fireplace or lighting a lightbulb, thus allowing light to be introduced to places where or at times when it would normally be absent. Sophisticated lasers, which stands for Light Amplification by Stimulated Emission of Radiation, allow light to be generated that is extremely narrowly defined in color and direction [6]. During stimulated emission, introduced by Einstein in 1917, an atom or a molecule in the excited state is hit by an incident photon. This photon causes the exited atom or molecule to decay to a lower energy state. The energy difference is released as a photon with the same frequency, polarization and direction as the incident photon [7]. The first working laser was demonstrated in 1960 [8] and basically consisted of a Ruby gemstone, coated on two parallel sides with silver mirrors. Nowadays, many different types of laser light sources exist, which are not only used in advanced optical laboratories, but also find use in common household technologies, like in compact disc (CD) and digital video disc (DVD) players.

Light is an efficient way to carry information. To illustrate, on naval ships morse codes are sent to communicate with nearby ships using light. In fiber-optic communication light is used to transmit data at high rates of several tera-bits per second and over long distances [9]. Furthermore, light is used in chemistry and sensing. For example, a well-known environmental pollutant such as lead ions can be detected in very small amounts by optode membranes [10]. In the presence of lead ions these membranes respond to light differently, an effect which can be measured and quantified for different ion concentrations. Light is also widely used in medicine. One example is photodynamic therapy where light-activated drugs are used to treat various diseases [11]. Using light enables the specific targeting of the area that is to be treated, e.g., a bronchial cancer tumor [12]. These examples demonstrate several ways of how light is used and show devices that were fabricated to use light more efficiently.

In the 1980's it was realized that light can be controlled in an intricate way by nanostructures, hence the term "nanophotonics". For instance, two groups demonstrated that multiple scattering of light can lead to intricate interference phenomena $[13,14]$. Of particular interest are three-dimensional 


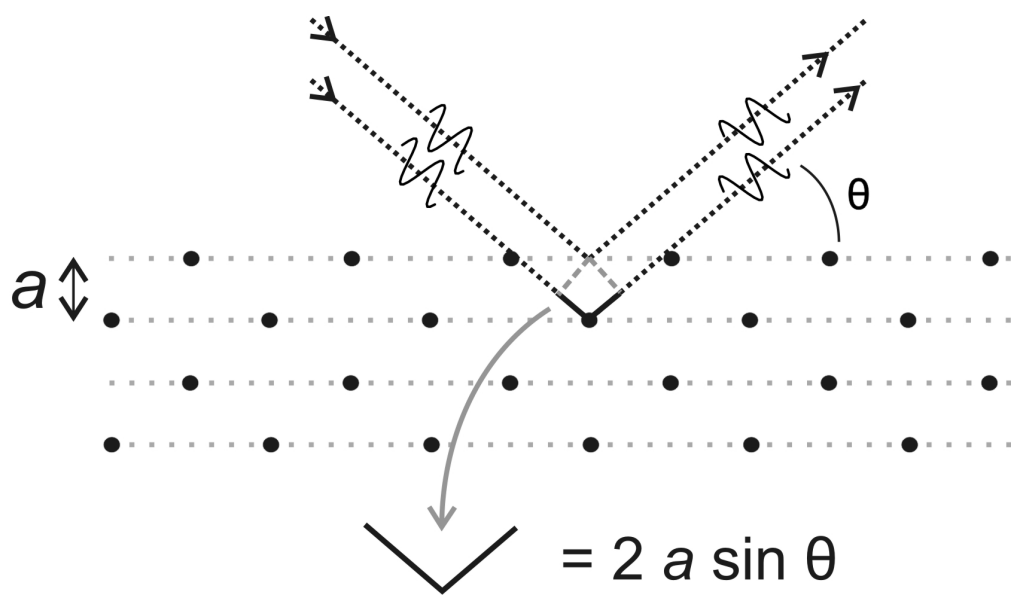

Figure 1.1: Representation of the Bragg condition. Incident light is reflected by the consecutive lattice planes (black circles connected by grey dotted lines). The extra optical path length for reflection by a next plane is $2 \cdot a \cdot \sin (\theta)$. If the wavelength divided by the effective index of refraction $\left(\lambda / n_{\text {eff }}\right)$ equals that value, the waves interfere constructively which gives rise to a peak in measured optical reflectivity spectra.

photonic crystals. In the ultimate three-dimensional photonic crystal with a band gap certain frequencies of light are forbidden from existing, which makes them the supreme devices to control light. Originally it was predicted that spontaneous emission of light is forbidden in these structures [15] or that light is localized [16]. Another intriguing aspect of photonic crystals is that by adding an embedded microcavity photons can be stored or slowed down [17]. Furthermore, photonic crystals are used to "mold the flow of light" $[18]$.

\subsection{Photonic crystals}

A photonic crystal is a periodic structure made from two different materials that are periodically alternated over length scales in the order of the wavelength of light $[15,16]$. Typically one material is air and the other has a high index of refraction, e.g., a semiconductor. Light with certain wavelengths $\lambda$ and wave vectors $k^{1}$ cannot propagate in such a structure because of Bragg diffraction [19]. This is the condition where light that is reflected

\footnotetext{
${ }^{1}$ The wave vector $k$ describes the direction of light. The vector magnitude is equal to the
} wave number, i.e., the reciprocal of the wavelength. 
off the lattice planes in the crystal interferes constructively. The Bragg condition was first described for x-rays, but is also valid for light. The Bragg condition is schematically depicted in Figure 1.1. For light, the wavelength $\lambda_{\text {Bragg }}$ at which constructive interference occurs can be calculated according to:

$$
n \cdot \lambda_{\text {Bragg }}=2 \cdot n_{\text {eff }} \cdot a \cdot \sin (\theta),
$$

with $n$ the order of reflection, $n_{\text {eff }}$ the effective refractive index of the crystal [20], $a$ the lattice spacing and $\theta$ the angle of the incident light. In case of a broadband (white light) reflectivity measurement on such a photonic crystal, the Bragg condition appears as a peak in the spectrum, also referred to as stop band. The Bragg condition (equation 1.1) corresponds to a wave vector $k=\pi / a$ for incident light perpendicular to the consecutive lattice planes.

The frequency $\omega$ of light of a certain wavelength $\lambda$ depends on the index of refraction of the propagating medium $n_{\text {med }}$ according to:

$$
\omega \cdot \lambda=2 \pi \frac{c}{n_{m e d}},
$$

with $c$ the speed of light. This relation is equivalent to:

$$
\omega=\frac{c}{n_{\text {med }}} \cdot k,
$$

which is the dispersion relation in a homogeneous medium, see the dashed diagonal line in Figure 1.2. At the Bragg condition $(k=\pi / a)$ there exist two standing waves in a photonic crystal: one in the high index of refraction material and one in the low index of refraction material [18]. These standing waves have the same wavelength, but since they reside in different materials this results in different frequencies of light for both materials, see equation 1.2. These two frequencies form the edges of the stop gap $\Delta \omega_{\text {gap }}$ of the photonic crystal, see Figure 1.2. The center of the stop gap is the central frequency $\omega_{\text {gap }}$. The stop gap disappears when the difference in the refractive index of both materials is zero, because the material is optically homogeneous (dashed diagonal line in Figure 1.2). The stop gap gets larger when the difference in refractive indices increases.

An important parameter of photonic crystals is the photonic strength $S$, defined as the polarizability per unit cell volume. The photonic strength $S$ is a measure of the interaction strength of photonic crystals with light [20]. A higher $S$ means that the crystal is more strongly interacting with light. By measuring $\omega_{\text {gap }}$ and $\Delta \omega_{\text {gap }}$, the photonic strength $S$ can be determined 


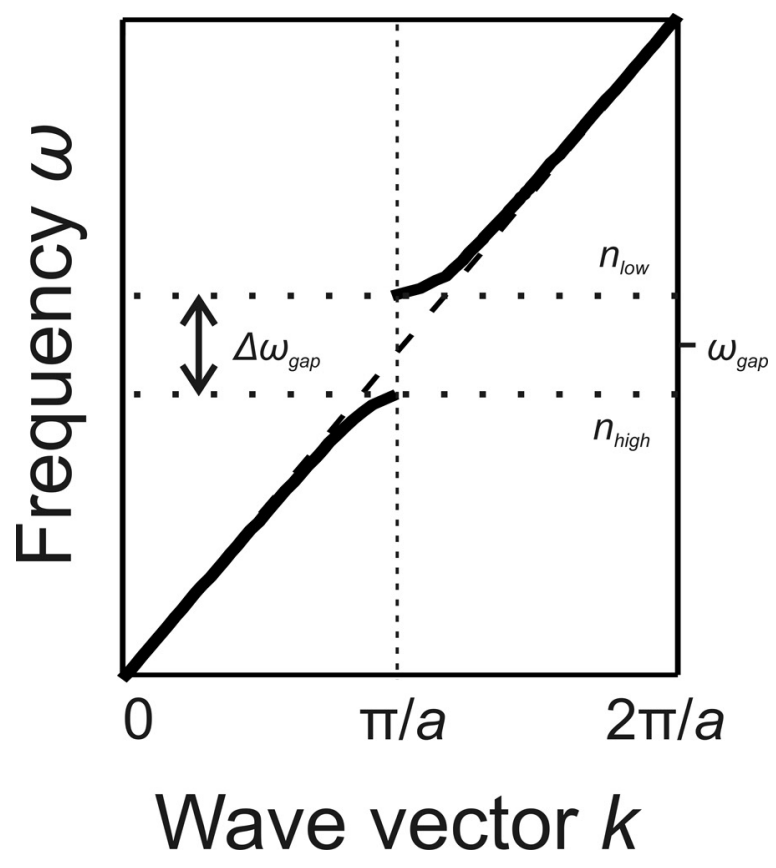

Figure 1.2: Dispersion relation of light in a photonic crystal (solid lines). At the Bragg condition $(k=\pi / a)$ a range of frequencies $\Delta \omega_{\text {gap }}$ exist for which propagation of light in certain directions is not possible: the so-called stop gap $\Delta \omega_{\text {gap }}$. The photonic strength $S$ of a crystal is defined as the width of the stop gap $\Delta \omega_{\text {gap }}$ divided by the center of the stop gap $\omega_{\text {gap }}$. Where this range of frequencies is determined experimentally, it is described with the term "stop band". The diagonal dashed line displays the dispersion relation in an optically homogeneous material which has no gap (equation 1.3).

experimentally:

$$
S=\frac{\Delta \omega_{\text {gap }}}{\omega_{\text {gap }}}
$$

From the photonic strength, a length scale can be derived: the Bragg attenuation length $L_{B}$. This length is a measure for the distance that light can penetrate into the photonic crystal before being reflected by the lattice planes. The Bragg attenuation length $L_{B}$ is equal to [21]:

$$
L_{B}=\frac{\lambda_{\text {Bragg }}}{\pi S},
$$

with $\lambda_{\text {Bragg }}$ the central wavelength of the measured stop band. A short 
Bragg attenuation length pertains to crystals with a high photonic strength $S$.

In photonic crystals the photonic strength $S$ can be written as [22]:

$$
S=3 \phi \frac{m^{2}-1}{m^{2}+2} 3 j_{1}(G R),
$$

with $\phi$ the volume fraction of high refractive index material, $m$ the refractive index contrast between high $\left(n_{\text {high }}\right)$ and low $\left(n_{\text {low }}\right)$ refractive index material:

$$
m=\frac{n_{\text {high }}}{n_{\text {low }}} .
$$

Assuming a photonic crystal of spheres arranged in a lattice, $G$ is the length of the dominant reciprocal lattice vector and $R$ the radius of the spheres. In $f c c$ crystals, the 111 reciprocal lattice vector is the dominant one. $j_{1}$ is equal to:

$$
j_{1}=\frac{\sin (u)-u \cos (u)}{u^{3}},
$$

with $u=G R$ proportional to the radius $R$ of the spheres and inversely to the lattice parameter $a$ of the photonic crystal. From equations 1.6, 1.7 and 1.8 it is obvious that the photonic strength $S$ of a crystal depends strongly on its structural and material properties. In particular we note that the photonic strength increases with the refractive index contrast $m$. This is the motivation for choosing high index of refraction materials, in our case silicon (refractive index $n_{S i} \approx 3.5$ ), to make photonic crystals.

Whereas a stop gap is defined in one direction only, in a photonic band gap crystal a range of forbidden frequencies exist for all directions in three dimensions. In order for a photonic band gap $^{2}$ to appear, stop gaps from different lattice planes in the crystal overlap and couple [23]. For this to occur, the photonic strength $S$ of the crystal, and thus the refractive index contrast $m$, must be high enough. The rule of thumb is that the photonic strength should exceed $S \geq 0.15$ to 0.20 for a photonic band gap to appear. For example $m \geq 2.8$ is required for a photonic band gap to appear in an $f c c$ inverse opal with $\phi=24 \%$ [24]. In this thesis, photonic crystals are qualified by the width of their (calculated) photonic band gap. We aim to obtain three-dimensional photonic crystals with as large as possible band gaps.

\footnotetext{
${ }^{2}$ In case of photonic band gap, $\omega_{\text {gap }}$ and $\Delta \omega_{\text {gap }}$ do not refer to stop gap properties, but refer to the central frequency of the photonic band gap and the width of the band gap, respectively.
} 


\subsection{Fabrication of three-dimensional photonic crystals}

Many different types of three-dimensional photonic crystals have been conceived, but of particular interest are those that potentially provide three dimensional photonic band gaps. Such structures offer ultimate control of light in all three dimensions simultaneously and therefore many research groups are studying their fabrication.

\subsubsection{Inverse opals}

Among the first structures considered as promising photonic crystals were $f c c$ crystals of colloidal spheres $[15,16]$. These crystals are routinely obtained by different methods of self-assembly [25-28]. Two types of $f c c$ crystals of spheres are discriminated: a) colloidal crystals which consist of separate spheres that perform Brownian motion while suspended in a liquid (typically water with $n_{\text {low }}=1.33$ ) and b) artificial opals where the spheres are immobilized as they are in close-packed contact and surrounded by air ( $n_{\text {low }}$ $=1$ ) . The reduced index of refraction $n_{\text {low }}$ in opals increases $m$ and thus the photonic strength $S$.

Artificial opals made from self-assembled colloidal spheres are of considerable scientific and technological interest as photonic crystals, as components of light sources, solar cells, and chemical sensors, as well as in the field of plasmonics [29-31]. Although colloidal crystals and artificial opals are interesting as photonic structures in their own right, see references [32-35], they cannot form a photonic band gap due to the limited photonic strength of these crystals: for an artificial opal of polystyrene spheres $S \approx 6 \%$, whereas for photonic band gap formation the photonic strength should be higher: $\mathrm{S} \geq 20 \%[21]$.

Although it was suggested that it is impossible to obtain a band gap in $f c c$ photonic crystals [36], it was later realized that in inverse air-sphere crystals it is possible to obtain a photonic band gap [24]. Inverse air-sphere crystals consist of $f c c$ stacked spheres of air, embedded in a backbone of a higher index of refraction material. These inverse artificial opals can be obtained by using $f c c$ colloidal sphere crystals as a template and filling them with a high index of refraction material ${ }^{3}$, followed by removal of the template. Fabricated examples include inverse opals of metal oxides like titanium dioxide [38, 39], of carbon [40], of CdSe [41], and even of metals [42]. In inverse opals the index of refraction contrast must exceed $m=2.8$ in order to obtain a

${ }^{3}$ Alternative templates for inversion can be obtained by, e.g., holographic lithography [37]. 
photonic band gap [24]. A material that meets this requirement is silicon with a refractive index of $n_{S i} \approx 3.5$. Consequently, inverse opals of silicon [43-45] are promising candidates to display a photonic band gap. By optical reflectivity experiments, stop bands in multiple directions were observed corresponding with the predicted frequency, suggesting that a photonic band gap is present in these crystals. Unfortunately, even for the relative high index of refraction material silicon, the maximum width of the band gap in $f c c$ inverse opals remains limited to around $5 \%$ predicted using a hard sphere model [24]. The relative width of the band gap increases to $12 \%$ when the crystals are considered as they are formed during fabrication [21], i.e., with a reduced filling fraction of high index material in shells and air spheres with windows connecting them with each other [46]. Regrettably, the photonic band gap in $f c c$ inverse opals is very sensitive to fabrication induced disorder [47] which limits the usefulness of these structures when large band gaps are required.

\subsubsection{Simple-cubic photonic crystals}

Different varieties have been suggested of three dimensional photonic crystals with a simple-cubic lattice. Among the proposed building-blocks are spheres, air-spheres, square rods, circular rods, and many other modifications [48-50]. Examples of simple-cubic crystals are beautiful air-sphere [51] and square rod crystals [52]. Unfortunately the maximum width of the band gap of simple-cubic photonic crystals remains limited: around 5\% was predicted for air-sphere crystals [53] and in reference [50] a maximum width of the band gap of $13 \%$ is reported for an optimized geometry (here a $2^{\text {nd }}$ order gap has opened, the width of the $1^{\text {st }}$ order gap is around $7 \%$ ). Unfortunately, we can expect these $2^{\text {nd }}$ order band gaps to be sensitive to fabrication induced disorder, similar to the band gap in $f c c$ air-sphere crystals. In reference [53] the band gap properties were calculated for some deviations in air-sphere simple-cubic crystals. There it appears that the frequency of the band gap alters significantly when deviations are introduced, which is undesirable since most potential applications rely on fixed wavelengths or frequencies.

\subsubsection{Diamond-like photonic crystals}

The so-called diamond-like crystal structure has received a great deal of attention because of its large calculated width of the band gap, and the property that the photonic band gap already opens at a lower index of refraction contrast of $m>1.9$ compared to $m \geq 2.8$ for $f c c$ air-sphere crystals. The 
use of diamond lattices in photonic crystals was introduced in reference [36] and reviewed in reference [54]. Interestingly, diamond-like photonic crystals were introduced because the authors concluded that a band gap could not open in $f c c$ air-sphere crystals of silicon. This conclusion was erroneous, as was shown in reference [24]. Nevertheless, diamond-like photonic crystals promise large band gaps.

An early type of diamond structured photonic crystals was proposed in 1991 [55]. This structure, which is now referred to as "Yablonovite" after its inventor, is obtained by milling or etching pores in separate steps in three directions. In 1995 such crystals were fabricated in GaAs and GaAsP [56], but they are unfortunately rather thin. In a subsequent study, similar crystals were shown to be photonic [57], but a band gap remains to be demonstrated. The structure is revealed to be sensitive to structural errors introduced during fabrication (tapered instead of cylindrical pores). For a "Yablonovite" crystal made from silicon, the calculated band gaps have a relative width of up to $19 \%$. In references $[58,59]$ a "Yablonovite" structure is fabricated using a combination of chemical etching and focused ion beam milling. This beautiful structure shows promising reflectivity peaks around $\lambda=3 \mu \mathrm{m}$. However, applying focused ion beam milling only allows the fabrication of crystals with a small volume and tapered pores, which are severe disadvantages.

Diamond-like woodpile structures were introduced in reference [60]. They are fabricated by stacking layers of dielectric rods on top of each other in sequential steps. The fabrication of woodpile photonic crystals was reported in references [61-63]. Although these structures are very impressive, they consist of stacked and rectangular touching rods, which means that the maximum photonic strength is limited to around $18 \%$ for structures in silicon [60]. In addition, the "layer-by-layer" fabrication of the woodpile crystals introduces alignment errors between each layer of rods, reducing the maximum obtainable photonic strength and limiting the achievable crystal volume. Furthermore, this method is time-consuming.

Two groups described "spiral diamond" crystals [64, 65], consisting of circular spirals. One of the techniques proposed to fabricate the structures is glancing angle deposition. Subsequent inversion of the square spirals with silicon yields a crystal with a calculated relative width of the band gap of around $24 \%$. Regrettably, this method is intricate and non-conventional. Furthermore it yields a structure with pores that are poorly interconnected, which imposes practical challenges like, e.g., how to homogeneously infiltrate the crystal with light sources in liquid carriers.

It has been attempted to fabricate diamond-like photonic crystals with 
dielectric spheres. Unfortunately, this is very complicated, see for example [66] where nanorobotic manipulation is used to obtain a pretty, but small five-layer crystal. Furthermore, crystals built from loose spheres are brittle and therefore unstable when, e.g., one wants to infiltrate them with light sources in a liquid carrier or solvent.

In reference [67] three-dimensional woodpile crystals were fabricated by a double-angled etching technique. By two consecutive cryogenic reactive ion etch steps of $45^{\circ}$ with respect to the wafer surface, at an angle of $90^{\circ}$ between the two etch directions, a thin photonic crystal was obtained. Unfortunately this method holds a number of fabrication related challenges which include the required extensive modification of the reactive ion etching equipment, compromised homogeneity of the etching result, and complex preparation of the mask for the second pore sets.

\subsubsection{Inverse woodpile photonic crystals}

Table 1.1 lists the relevant types of photonic crystals that have been discussed in this chapter. The final entry is the most promising type of diamond-like photonic crystals, which was introduced in [60]. These structures are referred to as inverse woodpile photonic crystals and consist of two geometrically identical arrays of pores perpendicular to each other in a high refractive index material, in our case silicon. They differ from woodpile crystal in the sense that the filling fraction of high refractive index material is optimized and that the pores may overlap, which is required to obtain a maximum band gap. Inverse woodpile photonic crystals are very interesting because of their conceptual ease of fabrication by only etching in two directions and their high photonic strength, resulting in a broad band gap with a relative width of more than $\left(\Delta \omega_{\text {gap }} / \omega_{\text {gap }}\right)=25 \%[60,68]$.

In reference [68] a method was proposed to fabricate such inverse woodpile structures by using macroporous silicon and subsequently focused ion beam milling. Later, a crystal was obtained using the proposed method [69], which was one of the first fabricated inverse woodpiles. It was pointed out that the structure was misaligned, which results in a reduced calculated width of the band gap. A reflectivity spectrum was shown with a $>40 \%$ reflectivity peak. This peak corresponds to a calculated stop gap in the direction that was measured. This result shows that these crystals are still reasonably strongly photonic.

Recently an alternative method was described to fabricate similar crystals [70]. This method involves direct laser writing [71] and silicon dioxide assisted deposition of polycrystalline silicon. Optical transmittance and re- 


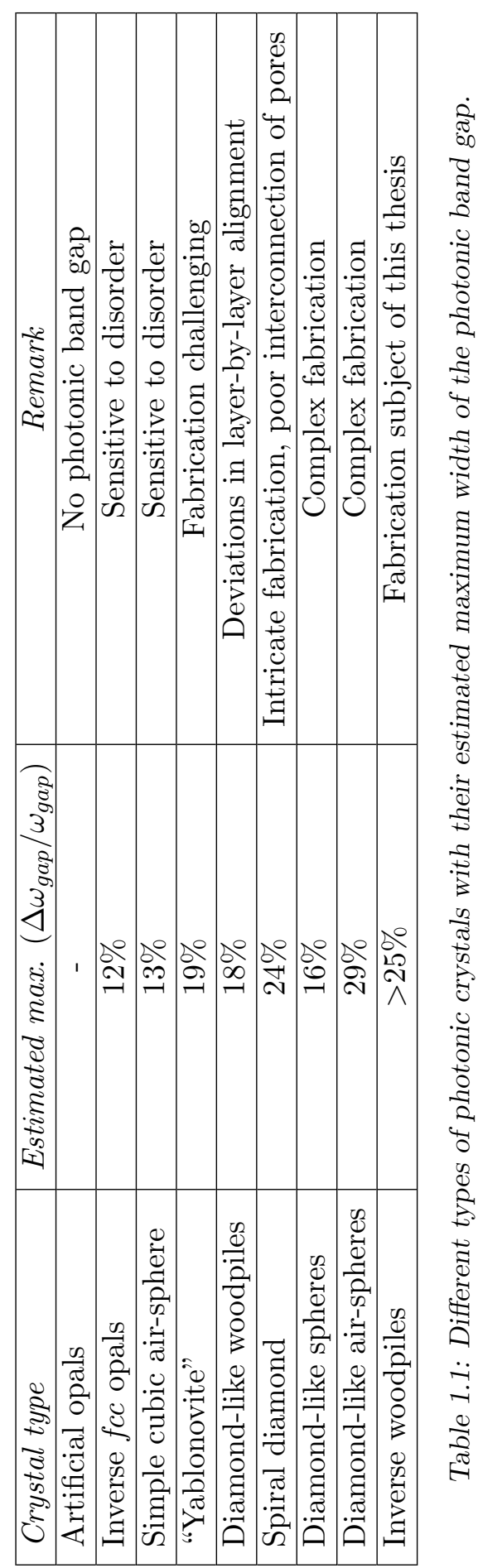


flectivity experiments show that this structure is indeed strongly photonic. However, the optical experiments were performed along only one direction and hence do not confirm whether a photonic band gap exists in this structure. With further calculations it is predicted that the structure can be improved to obtain relative widths of the band gap of $\left(\Delta \omega_{\text {gap }} / \omega_{\text {gap }}\right)=18 \%$, which is unfortunately well below the predictions in $[60,68]$. The pores in these crystals have an inherent strongly elliptical cross-section, with aspect ratios of approximately 1.5, which may very well be at the basis of the reduced maximum widths of the band gap ${ }^{4}$. In addition, although the fabrication method used to obtain the crystal is very impressive, it is very intricate and non-conventional.

These examples show that several inverse woodpile crystals have successfully been fabricated. However, in none of these cases was a photonic band gap convincingly demonstrated. We surmise that this is due to limitations of the applied fabrication methods which impose restrictions on the quality, and thus photonic strength $S$ of the crystals that were obtained and in some cases also on crystal size. The main part of this thesis discusses the development of alternative fabrication routes to inverse woodpile photonic crystals. We aim to use Complementary Metal Oxide Semiconductor (CMOS) industry compatible methods with as little as possible (two) etching steps to achieve as large as possible structures, where the crystals are carved out of crystalline silicon.

\subsection{Optical cavities in a three-dimensional photonic crystal}

One of the great promises of nanophotonics is the possibility that light is trapped in optical cavities inside three dimensional photonic band gap crystals. An optical cavity in a photonic crystal can be manufactured by either adding or removing high-refractive index material, which yields a donor or an acceptor, respectively $[15,72,73]$. The mirrors of the optical cavities are formed by the surrounding photonic crystal with a band gap. It has been predicted that light is trapped in such a photonic crystal cavity within a tiny volume as small as $1 / 100^{\text {th }}$ of a wavelength cubed [73, 74], therefore the name "nanobox for light" is proposed for such an unusual optical cavity [75]. It is

\footnotetext{
${ }^{4}$ Some ambiguity exists between references [60,68], as in [60] it is claimed that crystals made from pores with elliptical cross-sections would have the largest band gap, whereas in [68] is claimed that this is the case for cylindrical pores. The analysis in [70] supports the conclusion that pores with elliptical cross-sections are less favorable compared to cylindrical pores.
} 
a challenge to embed such controlled point defects inside three-dimensional photonic crystals to achieve functional systems [76, 77].

An excited light source placed near an optical cavity is allowed to emit one photon, yet the photon remains localized, which leads to novel quantumoptical phenomena [17]. Adding a tiny amount of matter to a single nanocavity drastically changes its optical properties [78], thus yielding a highly sensitive sensor. Furthermore, a tiny particle that moves inside a hollow optical cavity can be tracked with a high spatial resolution, hence the cavity serves as a high-resolution microscope [79]. In a different approach, focused ion beams can be used to selectively deposit materials including noble metals gold or platinum in such nanocavities. Noble metals are excellent substrates for self-assembled monolayers that offer chemical selectivity [80]. In this fashion size-selectivity, determined by the properties of the cavity and by the pores in the photonic crystal, can be combined with chemical selectivity introduced by the self-assembled monolayer [81]. If arrays of optical cavities can be achieved, one-dimensional rows that could serve as optical waveguides could be obtained [82-84], and even three-dimensional arrays that could eventually shuttle quantum information encoded on single photons.

The first experimental observations of donor and acceptor modes in $f c c$ crystals are reported in reference [72] for microwave frequencies. Examples of fabricated defects on a micrometer scale include the incorporation of point defects in woodpile structures $[85,86]$. We do not pursue such cavities because of the challenging layer-by-layer nature of the fabrication process, which induces alignment errors and consequently leads to reduced photonic strengths $S$ and lower cavity quality factors $Q$. Large area modifications have been written in the surface of polymeric opal films, see for examples references [87] and [88]. It is likely that these defects are too large to confine light. In addition, large defects were fabricated in silicon inverse air-sphere crystals using two-photon polymerization [89]. The resulting defects were channels with waveguide-like properties, but not point defects. For woodpile photonic crystals a method was proposed to obtain an optical cavity with a high quality factor $Q$ by combining a unit cell modulation (planar defect) and a waveguide (line defect) [90]. It would be interesting to study whether this method is applicable to inverse woodpile photonic crystals. To date, however, all (inverse) woodpile crystals with cavities are relatively small or thin, hence it is worthwhile to consider cavities in large crystals such as (inverse) opals. The challenge to make cavities in (inverse) opals is of course that these systems are the result of self-assembly, hence it is impossible to predetermine where a cavity can be placed, and it is challenging to predetermine the cavities' size. Therefore we explore in this thesis a method to fabricate single 
defects and arrays of defects at well-defined positions and with controllable defect-volumes in self-assembled artificial opals.

\subsection{Applications of photonic crystals}

Nowadays, photonic crystals are applied in many ways to control light and without a doubt many more applications will be realized in the future. While it is impossible to list all these (potential) applications here, we will provide a small sampling of the possibilities:

- Many efforts are devoted to the fabrication of thresholdless cavity lasers $[17,91,92]$. Lasers are characterized by their spontaneous emission coefficient $\beta$, which is described for cavity lasers, see e.g. reference [92], and for random lasers [93]. A higher coefficient $\beta$ means that more spontaneous emission contributes to the laser mode. In case $\beta=1$, the laser is thresholdless which means that all input power is converted to laser light. Thresholdless cavity lasers are interesting in, e.g., miniaturized devices, because of their high efficiency and lower noise. Fabrication of such lasers rely on optical cavities with high cavity factors $Q$ to increase the spontaneous emission coefficient $\beta$ and photonic crystals are expected to enable thresholdless lasers [15]. Indeed, lasers have been made using photonic crystals, see for example reference [94], where an impressive surface-emitting blue-violet laser is presented. In another example it has been shown how the emission wavelength depends on the lattice parameters of two-dimensional photonic crystal laser systems [95]. However, in these examples the lasers do possess a threshold value and to the best of our knowledge, thresholdless photonic crystal based lasers have not yet been demonstrated.

- A different type of lightsource that may be fabricated using photonic crystals are high-efficiency light emitting diodes (LED's) [96]. Light emitting diodes may be improved by using photonic crystals by two different methods. The first method is changing of the photonic band gap properties such that the spontaneous emission of light trapped in the device is inhibited, thus increasing the fraction of spontaneous emission in the emitted modes [97]. The second method is to use photonic crystals to more efficiently extract light from within the device [98]. In this case, the spontaneous emission properties of the light emitting diode remain unaltered. In particular the fabrication of light emitting diodes via the second method is widely researched, resulting in significant enhancements of emitted light [99, 100]. 
- Photonic crystals can be used as optical components, among which fibers [101], polarizing beamsplitters [102], and waveguides to control the propagation of light $[103,104]$.

- Photonic crystals are used for detection and sensing. Examples include detection of gas molecules [105], highly sensitive fluorescence detection [106], and the detection of single particles [107].

- Photonic crystals are used to change the behaviour of light sources that are placed inside photonic crystals, e.g., by changing the spontaneous emission rate [108-110] or by changing the color at which light sources emit [111]. Enhancing or inhibiting the spontaneous emission of light sources is at the basis of various photonic applications such as miniature lasers and light emitting diodes (see above), and solar cells $[112]$.

- (Optically) switching three-dimensional photonic crystals [113-115] allows the photonic strength of the structure to be altered in a controlled fashion. This allows, for example, dynamic control of the emission of lightsources that are inside the photonic crystal, e.g., the emission can be turned on or off.

- Ultimately, photonic crystals are predicted to contribute to all-optical computers $[44,96,116]$. Compared to traditional electronic computers, all-optical systems should be more energy efficient, faster and have larger bandwidths.

In our groups at the University of Twente and the FOM Institute AMOLF, photonic crystals are fabricated to study their optical properties, investigate the behaviour of embedded lightsources, and perform optical switching experiments. Furthermore, investigating (novel) fabrication techniques in order to obtain or modify photonic crystals is a primary interest of these groups as is evident by the subject of this thesis.

\subsection{This thesis}

To fabricate bulk inverse woodpile photonic crystals, a broad range of fabrication technologies and methodologies were investigated. Furthermore, a method was studied to obtain point defects inside opals and even in single colloidal nanospheres. The fabrication and optical characterization of the fabricated structures is described in this thesis. 
- In chapter 2 the inverse woodpile three-dimensional photonic crystals are introduced that we want to manufacture. The fabrication schemes are described which are used to obtain these crystals. An analysis is given of the effect of certain deviations from a "perfect" crystal on the photonic band gap.

- In chapter 3 the photo electrochemical etching of macropores in crystalline silicon is described. The setup that was built is introduced and the best results to date are shown and analyzed.

- Chapter 4 describes the fabrication of periodic arrays of deep nanopores with high aspect ratios in crystalline silicon. The pores were made with a reactive ion etching process, optimized for the formation of these deep nanopores. The optimization of the etching process is described in detail. A theoretical analysis is made of the main limitation on the maximum aspect ratios.

- Chapter 5 describes a holder wafer developed to enable the fabrication of three-dimensional photonic crystals using two-dimensional photonic crystal pore arrays as starting point. The fabrication and testing of the holder wafer is described and, using the holder wafer, a mask for etching is demonstrated.

- Chapter 6 presents a new method to realize single nanocavities and arrays of nanocavities in individual colloidal particles on the surface of artificial opals using a focused ion beam milling technique. The relation between defect size and milling time is studied. Optical microscopy experiments show the interaction of light with the fabricated nanocavities.

- In Chapter 7 the optical reflectivity of our two- and three-dimensional crystals is studied and compared to calculated band structures. The fabrication is briefly described of three-dimensional photonic crystals made by focused ion beam milling of the deeply etched two-dimensional structures.

- Chapter 8 summarizes the work in this thesis and outlines possible future work. 


\section{Fabrication and optical properties of inverse woodpile photonic crystals}

\subsection{Introduction}

The most promising type of diamond-like photonic crystals are the inverse woodpile photonic crystals [60], see chapter 1 . These crystals consist of two geometrically identical arrays of cylindrical pores of air perpendicular to each other in a high refractive index material, in our case silicon, see Figure 2.1. Inverse woodpile photonic crystals are very interesting because of their conceptual ease of fabrication and high photonic strength, resulting in a broad band gap with a relative width of more than $(\Delta \omega / \omega)=25 \%[60,68]$.

The examples in chapter 1 showed that several inverse woodpile crystals have successfully been fabricated, but, in our opinion, none with a convincingly demonstrated photonic band gap. We attributed this to limitations of the applied fabrication methods. We propose to fabricate inverse woodpile photonic crystals by using well-known, and in the $\mu$ m regime well developed fabrication techniques. By scaling-down these techniques to sub-micrometer dimensions, optimal structural dimensions and crystal quality are within reach. In order to make integration of our structures in silicon integrated circuits (IC) feasible, preferably all fabrication techniques are compatible with methods used in the complementary metal oxide semiconductor (CMOS) industry.

In this chapter we discuss the fabrication schemes that we use to obtain these structures. In addition, an analysis is given of the effect of certain deviations from a perfect crystal on the photonic band gap by analyzing literature, and by performing calculations. 
Chapter 2. Fabrication and optical properties of inverse woodpile photonic crystals
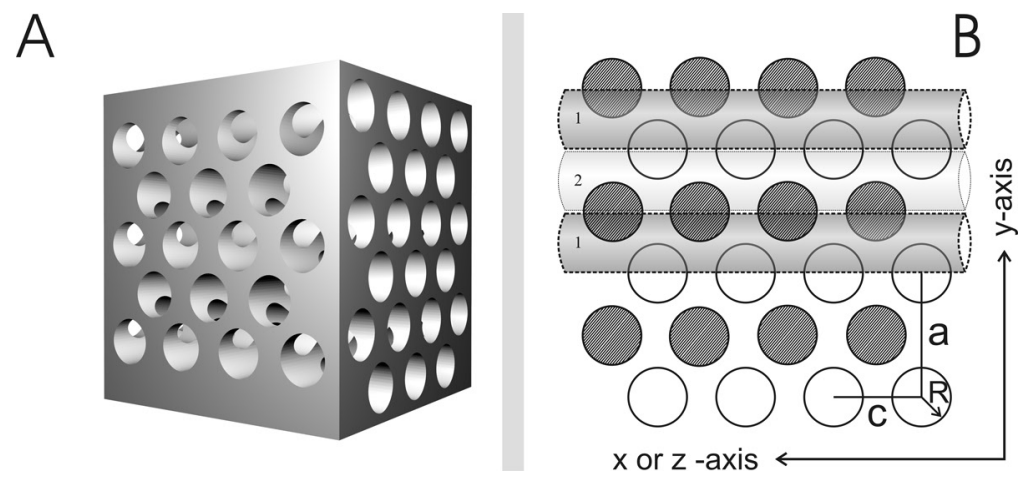

Figure 2.1: A) Three-dimensional representation of a cubic inverse woodpile photonic crystal. The alignment of the pores between the pores of the other set can be clearly seen, as well as the overlap of the pores. Figure prepared by T. Rozendal. B) Face-on view of the inverse woodpile photonic crystal. The centers of the perpendicular cylinders are aligned exactly between rows of cylinders of the other set. The individual pore sets have a centered rectangular lattice symmetry, with lattice parameters $(a / c)=\sqrt{2}$. An optimal photonic crystal is achieved when the ratio of radius $R$ to lattice parameter a equals 0.24 .

\subsection{Geometry of the cubic inverse woodpile structures}

What we define in this chapter as a "perfect" cubic inverse woodpile crystal consists of two perpendicular, geometrically identical, sets of cylindrical pores as first proposed by Ho et al. [60]. In Figure 2.1(A) the important crystal dimensions pore radius $R$, and lattice parameters $a$ and $c$ are indicated. The individual pore sets have a centered rectangular lattice symmetry [117], with $(a / c)=\sqrt{2}$. The combination of the two sets results in a threedimensional structure with a face-centered-cubic lattice. The centers of one perpendicular pore set are aligned exactly between rows of pores of the other set, see Figure 2.1(B). The pores of the two sets overlap, as is clearly visible in Figure 2.1(A). When the ratio of $(R / a)$ is equal to 0.24 , a crystal is obtained with a maximized photonic band gap. Furthermore the pores are perfectly cylindrical and not tapered, i.e., not conical. The pore walls are smooth to avoid any unwanted scattering of light. Ideal dimensions are $a=680 \mathrm{~nm}, c=481 \mathrm{~nm}$, and $R=163 \mathrm{~nm}$. This yields a crystal with a large photonic band gap centered at frequency $\omega=8290 \mathrm{~cm}^{-1}\left(\lambda_{\text {gap }}=1210 \mathrm{~nm}\right)$, which is detectable in our optical setups. Moreover, the band gap has a predicted relative width of more than $(\Delta \omega / \omega)=25 \%$ [60, 68], which does readily overlap with the telecommunication range. 


\subsection{Fabrication scheme}

A pictorial overview of the fabrication route towards inverse woodpile photonic crystals is given in Figure 2.2. The fabrication is started by etching the first pore set, shown in (A). Then a piece of the etched sample is retrieved (B). To obtain a three-dimensional cubic inverse woodpile crystal, the etched sample needs to be tilted by $90^{\circ}$, see Figure $2.2(\mathrm{C})$, and held in a separately fabricated "holder wafer" that is proposed here. This holder wafer will enable accurate alignment of the second pore set with respect to the first. Subsequently mask deposition, spinning of resist, and lithography can proceed. After mask alignment and preparation, etching of the second pore set can commence.

Different techniques could be used to etch the two separate pore sets 1 and 2, see Table 2.1. In particular, etching of the second set of pores is challenging, as it is difficult to correctly align it between the first set of pores. Furthermore, to obtain a good photonic crystal, it is necessary that the pores are perpendicular to each other, which imposes further challenges to the alignment of the second pore set to the first. To facilitate correct alignment of the two pore sets, a holder wafer is separately fabricated. For the different fabrication routes Table 2.1 lists whether mounting of the sample in a holder wafer is needed in order to align the second pore set to the first.

Several options are available in cases where a lithographic process is used to prepare masks that define the positions of the fabricated pores. Where ebeam- [118] or laser interference lithography [119] are often used in research, deep UV scan and step lithography is the most used method in the complementary metal oxide semiconductor (CMOS) industry. Hence we have chosen to use that technology. The main advantages of deep UV lithography are that it is fast and allows the patterning of large surfaces.

The preferred route is to fabricate crystals combining photo electrochemical etching and reactive ion etching (route 1). Route 1 is the preferred way as this leads to structures with the largest crystal volumes. This is due to the high aspect ratio pores that can be achieved with photo electrochemical etching, see references [120-122]. While developing this route is the main target of this thesis, we will also describe developments of several alternative routes, as these may yield successful results in a shorter time or provide prototypes to route 1 . Unfortunately it is not possible to fabricate crystals by photo electrochemically etching both pore sets (route 6). As soon as during etching a second set of pores would cross the first, the etchant would pour into the already present pores and these would then be etched sideways and destroyed. Alternatively, inverse woodpile crystals can be obtained by 
Chapter 2. Fabrication and optical properties of inverse woodpile photonic crystals

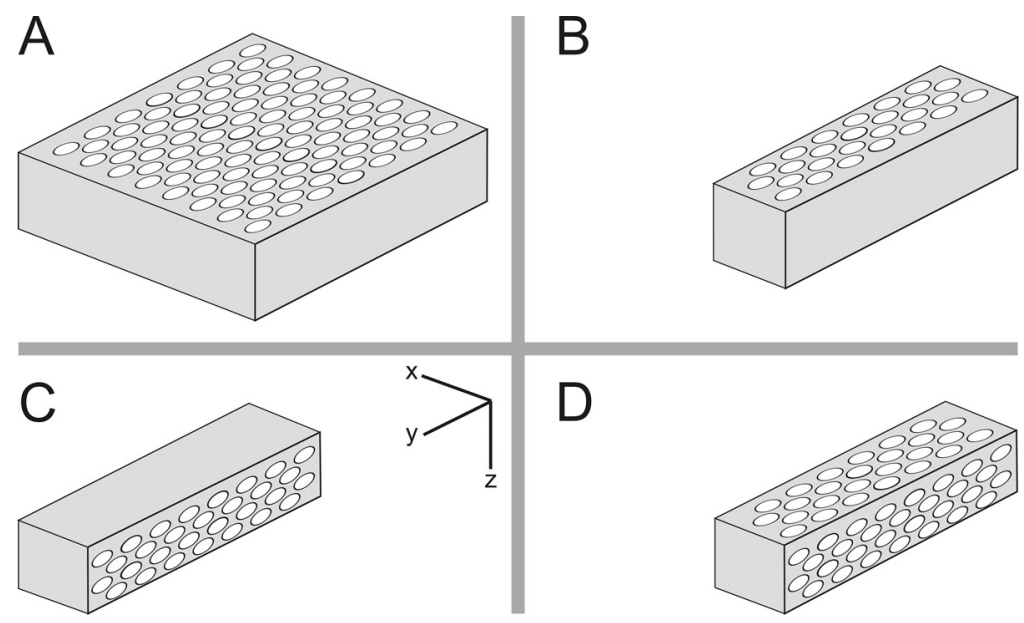

Figure 2.2: Schematic overview of how an inverse woodpile photonic crystal is obtained. A) A large area is etched with the first pore set. B) A piece of the sample is retrieved to be further processed. C) The sample is tilted by $90^{\circ}$. In the upper right corner of this facet we define $x, y$, and $z$ axis with respect to the pore set directions. D) After careful alignment to the first pore set, the second pore direction is etched.

preparing both pore sets with a reactive ion etch process (route 2). This has the advantage that the same fabrication method is used for both pore sets, but the maximum obtainable crystal volume is less than compared to route 1 because reactive ion etching yields less deep pores compared to photo electrochemical etching. Furthermore, focused ion beam milling can be used to etch pores (routes 3 to 5). Using a focused ion beam eliminates the use of a wafer holder, as all alignment is performed in the workstation. In addition, there is no mask preparation needed, as focused ion beam milling is a maskless method. However, focused ion beam has the disadvantages that only a small area can be processed, significantly reducing the maximum obtainable crystal volume, and that the process yields tapered pores, reducing the homogeneity of the photonic crystal. Nonetheless, focused ion beam milling is useful as a fabrication technique, as it allows rapid prototyping. We will show photonic crystals fabricated via route 4 , reactive ion etching and subsequent focused ion beam milling, in chapter 7 .

Fabrication route 1 potentially delivers photonic crystals with the largest volume. This fabrication route splits up in three parts: 1) deep pores fabricated by photo electrochemical etching, 2) deep pores fabricated by reactive ion etching, and 3) fabrication of the holder wafer. In the next section we 
ㄱ.

के

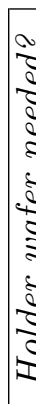

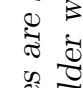

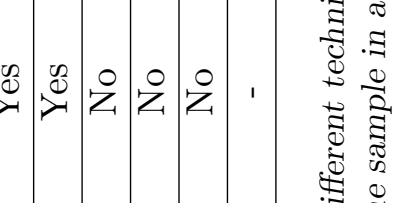

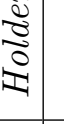

A

ฉ.

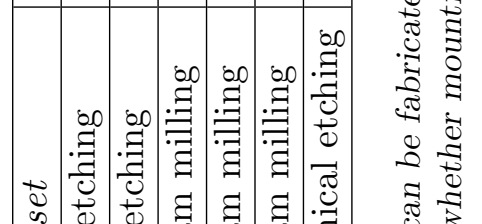

$$
\text { รั }
$$

है

\%

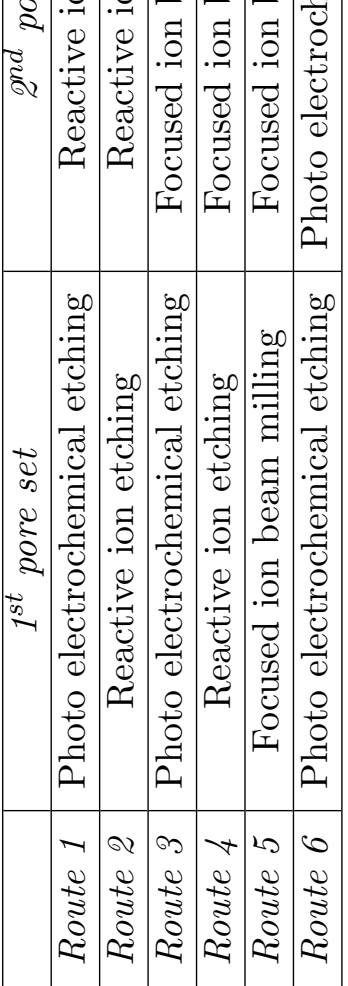

ฮี

蛋

.

, t)

0.

क्ष

¿

क⿺

..

สี สี

穿

인

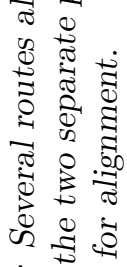

ㄱํ류

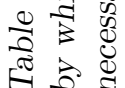


Chapter 2. Fabrication and optical properties of inverse woodpile photonic crystals

will discuss these three fabrication steps.

\subsubsection{Photo electrochemical etching}

By photo electrochemical etching with electrolytes containing hydrofluoric acid (HF), very deep and straight pores can be etched in silicon, see for example [120-122]. A scheme of how a wafer can be patterned, and subsequently etched to yield deep cylindrical pores is presented in Figure 2.3:

I) On a suitable n-type silicon wafer, silicon nitride $\left(\mathrm{Si}_{3} \mathrm{~N}_{4}\right)$ is deposited as mask material.

II) A resist is deposited on top of the silicon nitride.

III) The resist is patterned and developed to define the correct positions to yield a mask for the etching of the silicon nitride layer.

IV) The pattern in the resist is transferred to the silicon nitride layer.

V) After patterning of the silicon nitride layer, the remaining resist is removed.

VI) With the silicon nitride mask, inverted pyramid shaped pore tips are etched in the silicon wafer using a $\mathrm{KOH}$ solution [123].

VII) The remaining silicon nitride is removed and the pore tips are exposed.

VIII) Deep cylindrical pores are etched in the silicon wafer by photo electrochemical etching in a HF containing electrolyte.

In chapter 3 the patterning experiments $(I-V I I)$ are described in detail, as well as an analysis of our results of the photo electrochemical etching (VIII).

\subsubsection{Reactive ion etching}

Reactive ion etching is commonly used to obtain deep structures in silicon, see for example [124, 125]. A clear advantage of this technique over photo electrochemical etching is that pores can be etched in bulk silicon, but also through an already present pore set [67]. In this way a three-dimensional structure is fabricated. In this section we present a scheme how a wafer can be patterned, and etched to yield deep cylindrical pores, see Figure 2.4:

I) A layer of $50 \mathrm{~nm}$ chromium is deposited on a silicon wafer. 


\section{A) Photo electrochemical etching}

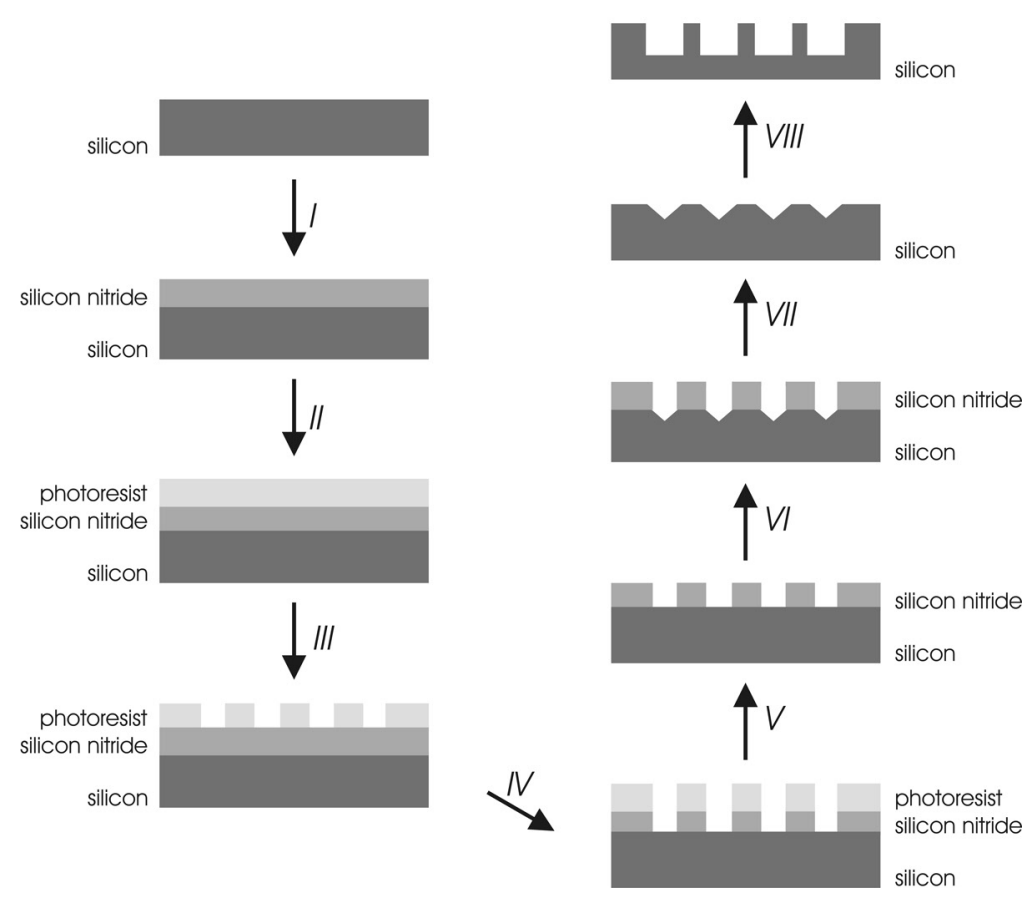

Figure 2.3: Outline of the patterning and photo electrochemical etching of a n-type silicon wafer. In short, a pattern of pore tips is prepared on the surface of the wafer using a silicon nitride mask and resist. With these pore tips as a starting point, deep pores are etched.

II) A resist is deposited on top of the chromium.

III) The resist is patterned and developed to define the correct positions to yield a mask for the etching of the chromium layer.

IV) The pattern in the resist is transferred to the chromium layer by chloride plasma based reactive ion etching.

V) With the chromium and resist mask, deep pores are etched in the silicon wafer using a Bosch-type deep reactive ion etching process [126].

VI) The remaining chromium and, if any, photoresist is removed from the wafer.

In chapter 4 a detailed description is given of the mask preparation $(I-I V)$ and the deep reactive ion etching of pores $(V)$. 
Chapter 2. Fabrication and optical properties of inverse woodpile photonic crystals

\section{B) Reactive ion etching}

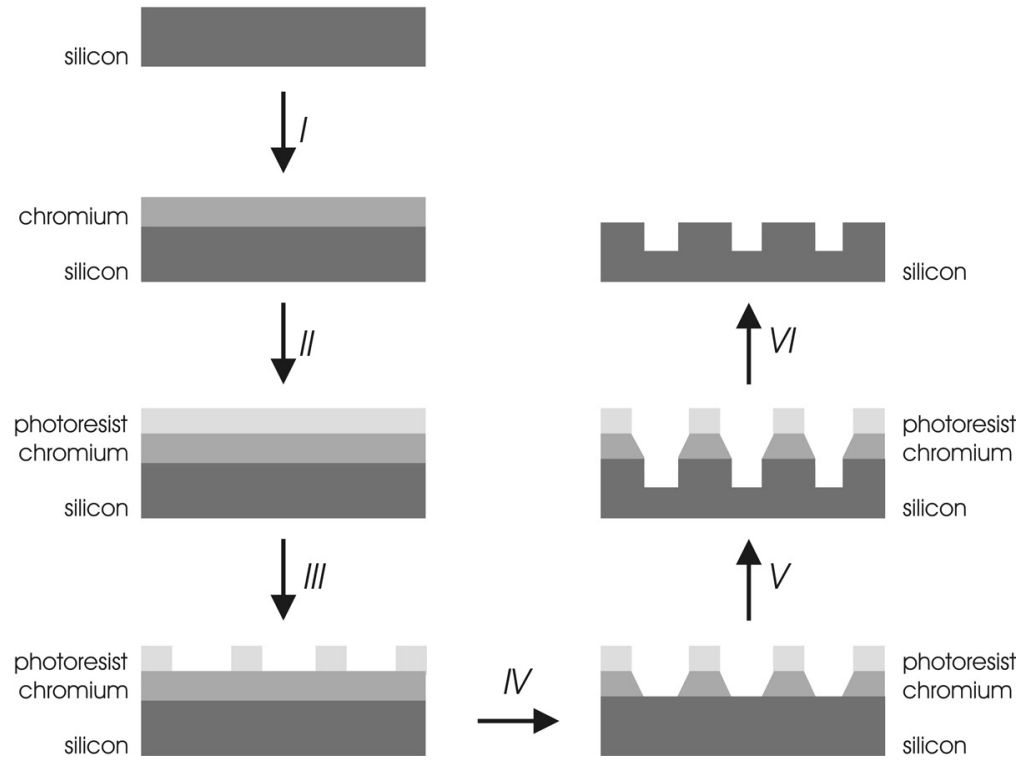

Figure 2.4: Outline of the patterning of a silicon wafer and subsequent deep reactive ion etching of pores. In short, on the surface of the wafer a mask of chromium and resist is prepared. Through this mask the pores are etched, after which the remaining mask is removed.

When a sample is etched with only the first set of pores, it already is an interesting two-dimensional photonic crystal. Therefore, chapter 7 describes reflectivity measurements on a crystal in which the first set of pores was etched with a reactive ion etching process.

\subsubsection{The holder wafer and mounting of tilted sample}

A process route was developed to fabricate a holder wafer in order to be able to correctly align the second set of pores to the first, see Figure 2.5:

I) Slots are cut in a double side polished silicon wafer using a laser cutting machine. Figure 2.5 shows a cross section and a topview of such a holder wafer.

II) The holder wafer is placed on top of a dummy wafer. Samples that are cut to size are tilted by $90^{\circ}$ and placed in the slots of the holder 
wafer. The surface of the sample that is going to be etched is placed in contact with the dummy wafer. In this fashion the dummy wafer accurately aligns the surface of the tilted sample parallel to the surface of the holder wafer.

X) A silicon wafer is selected and a thick layer of photoresist is spin coated on top of it. This creates a so-called "glue" wafer.

III) The "glue" wafer and the holder wafer are put on top of each other. Both are pressed together. By doing so, some of the photoresist is pressed into the voids between holder wafer and tilted sample. Subsequently the structure is placed into an oven to harden the photoresist. The sample and holder wafer are now firmly attached to each other.

IV) After hardening of the resist, the entire construction is flipped.

V) The dummy wafer is removed to expose the surface of the sample to be etched. Mask material can now be deposited on this surface and, after alignment, patterned by lithography.

\subsection{Deviations from a perfect cubic inverse woodpile crystal}

From a fabrication point of view it is important to know what the effect is of unintended deviations from a perfect crystal geometry on the photonic band gap. In this section we discuss a number of deviations that most frequently occur as a result of the manufacturing process.

The important structural dimensions of the inverse woodpile photonic crystals are the lattice parameters $a$ and $c$, and the radii of the two pore sets $R$, see Figure 2.1(B). In a perfect crystal, these dimensions are optimized such that the ratio of $(R / a)=0.24$ and the ratio of $(a / c)=\sqrt{2}$. Furthermore the two pore sets are flawlessly aligned. In such a structure the relative width of the band gap is more than $(\Delta \omega / \omega)=25 \%[60,68]$.

We recalculated photonic band structures as shown in reference [68] of such a perfect crystal with the MIT photonic bands package [127], see Figure 2.6(A). For these calculations the structure is considered to have a orthorhombic lattice symmetry. Figure 2.6(B) shows the Brillouin zone of the orthorhombic lattice. For $(R / a)=0.24$ and $(a / c)=\sqrt{2}$ our calculations confirmed a relative width of the band gap of $(\Delta \omega / \omega)=25.3 \%$, centered 
Chapter 2. Fabrication and optical properties of inverse woodpile photonic crystals

\section{C) Holder wafer and mounting of tilted sample}

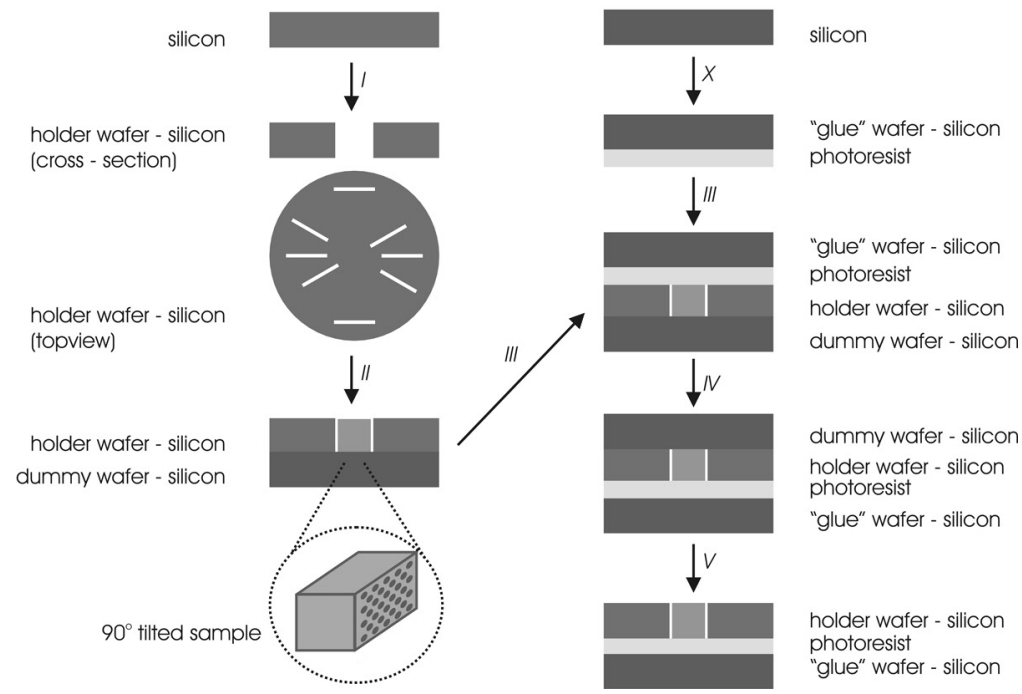

Figure 2.5: Outline of how a holder wafer is constructed and how tilted samples are placed inside this wafer. In short, by placing the samples into slots in the holder wafer and glueing both with photoresist, a solid attachment and proper alignment of sample surface and holder wafer surface are realized.

around frequency $=0.564[\omega a / 2 \pi c]^{1}$. Any deviation that occurs in the crystals will likely reduce the relative width of the band gap, and possibly affect the central frequency of the band gap. In the following sections we consider the following 5 types of deviations:

1 ) a radius $R$ to lattice parameter $a$ ratio different from 0.24 ,

2) a $\Delta y$ displacement along the y-axis between the two sets of pores,

3) angular misalignments,

4) tapered pores, and

5) different cylinder radii of the two sets of pores.

\subsubsection{Radius $\mathrm{R}$ to lattice parameter a different from $\mathbf{0 . 2 4}$}

When the radii of the etched pores deviate from the intended radius, the ratio of radius $R$ to lattice parameter $a$ is different from the optimal value of $(R / a)=0.24$. This is discussed in references [60,68]. They showed that

\footnotetext{
${ }^{1}$ In all our calculations with the MIT photonic bands package, we calculated bands 1 to 8 , with a $70 \times 100 \times 70$ grid and 73 k-points. The dielectric constant of silicon was chosen to be $\varepsilon_{S i}=12.1$.
} 

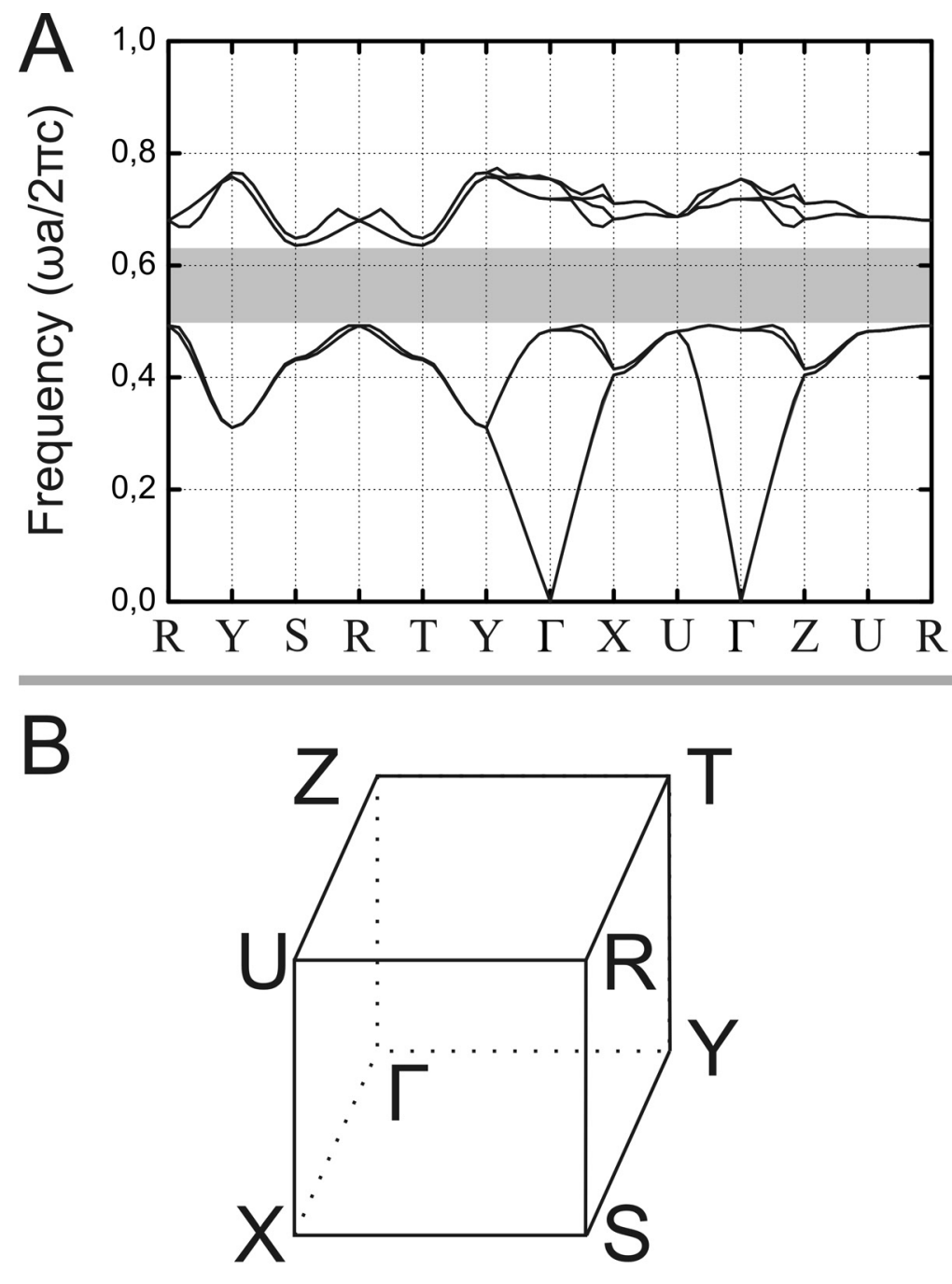

Figure 2.6: A) Calculated band structure of a perfect inverse woodpile photonic crystal. In such a crystal the radii $R$ of the two perpendicular pores sets are equal to 0.24 times the lattice parameter a, furthermore $(a / c)=\sqrt{2}$. The two pore sets are perpendicular aligned with respect to each other. The calculated band gap (grey bar) has a relative width of $(\Delta \omega / \omega)=25.3 \%$, centered around frequency $=0.564[\omega a / 2 \pi c]$. B) Brillouin zone of the orthorhombic lattice. Indicated are the 8 symmetry points $\Gamma, X, Y, Z$, etcetera. $\Gamma$ corresponds to $(0,0,0), X$ to $\left(\frac{1}{2}, 0,0\right), Y$ to $\left(0, \frac{1}{2}, 0\right)$, and $Z$ to $\left(0,0, \frac{1}{2}\right)$. The $\Gamma-X$ and $\Gamma-Z$ directions correspond to the directions parallel to the two sets of pores in the crystal. 
Chapter 2. Fabrication and optical properties of inverse woodpile photonic crystals

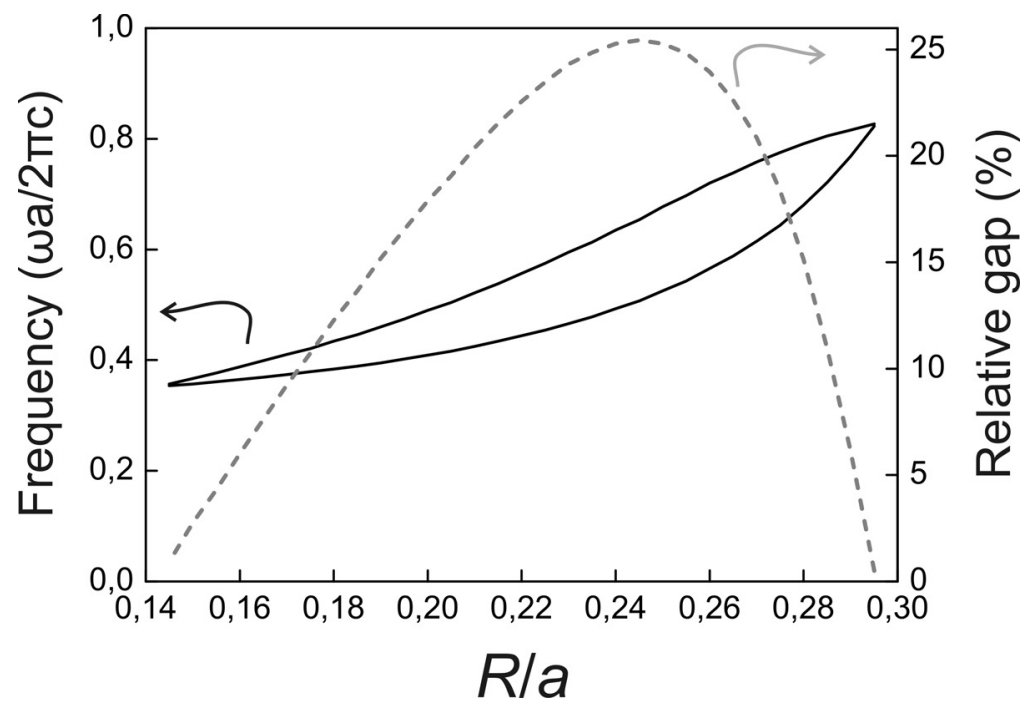

Figure 2.7: Calculated relative width of the band gap and frequencies of band gap edges for crystals with equal pore radii of the two sets of cylinders. $(R / a)$ was varied from 0.14 to 0.30 , with 0.005 increments. We find an maximum relative width of the band gap of $(\Delta \omega / \omega)=25.4 \%$ at a central frequency of 0.581 [ $\omega a / 2 \pi c]$ for $(R / a)=0.245$. For $(R / a)$ values increasingly further away from the optimal value, the relative band gap width is reduced (dashed line). The band gap edges (solid lines) move to larger frequencies with increasing $(R / a)$.

already at $(R / a)$ ratios of around 0.18 and 0.28 the relative width of the band gap has been reduced by half. At $(R / a)<0.14$ and $(R / a)>0.29$, there is no longer a band gap present. In order to obtain data with known increments of $(R / a)$ and known calculation settings and have an additional check of our computation method, we recalculated the band structures for the region $0.14<(R / a)<0.30$, see Figure 2.7.

The maximum relative width of the band gap of $(\Delta \omega / \omega)=25.4 \%$ is found for $(R / a)=0.245$. Here the central frequency of the band gap equals $0.581[\omega a / 2 \pi c]$. For $(R / a)$ values increasingly further away from the optimal value, the relative band gap width is reduced. The band gap moves to larger central frequencies with increasing $(R / a)$. We compared our data with the data in [68] and found good coincidence to within $(\Delta \omega / \omega)= \pm 1.8 \%$ in the entire calculated range (not shown in Figure 2.7). Thus, we conclude that our calculations are accurate.

Based on the results in [60] and [68] we initiated our fabrication towards $(R / a)=0.24$. We calculated an optimal relative width of the band gap at 
$(R / a)=0.245$. As the difference between these two values is very small, we have confirmed that $(R / a)=0.24$ was chosen correctly. To be able to use our calculations in practice, this ratio is considered optimal and we based our further calculations on that assumption.

The ratio $(R / a)$ directly relates to the volume fraction of silicon $\phi_{S i}$, i.e., increasing $(R / a)$ results in lower $\phi_{S i}$. At $(R / a)=0.245$, the volume fraction of silicon $\phi_{S i}=19.8 \%$, which means that the amount of high refractive index material is lower than the amount of low refractive index material. This is consistent with the rule of thumb "The optical path lengths in the high and low index materials should be equal, to enhance interference" that is generally applicable to photonic crystals.

\subsubsection{A $\Delta y$ displacement between the two sets of pores}

One of the alignment errors that may occur during fabrication is that the pores in the second set are not fabricated exactly in between the pores of the first pore set. This results in a $\Delta y$ displacement as illustrated in Figure 2.8 , where the second set is misaligned to the left or the right from its ideal position. In the Figure the first set of pores is pointing "into" the paper and the second set of pores runs parallel to the z-axis. Due to symmetry reasons, a misalignment to the left will have the same effect on the photonic band gap as a misalignment to the right by the same value. Such a $\Delta y$ displacement typically results from the lithographic process by which the second pore set is defined. Alternatively, it results from a misalignment during focused ion beam milling of the structures. It is impossible to obtain perfect alignment, and a small deviation from the perfect geometry is inevitable. In reference [128] the effect of this deviation on the band gap is discussed: a displacement of up to $50 \mathrm{~nm}$ results in a slight reduction of the relative photonic band width to $>90 \%$ of the original value. This means that the relative width of the band gap that remains is more than $(\Delta \omega / \omega)=22.8 \%$. When the $\Delta y$ displacement exceeds $50 \mathrm{~nm}$, the relative width of the band gap decreases rapidly. Since our alignment process is expected to perform much better than $50 \mathrm{~nm}$ precision, we do not expect this deviation to contribute significantly to a reduced relative width of the band gap.

\subsubsection{Angular misalignments}

When the sample, which has been etched in the first direction, is positioned in the holder wafer, it may be angularly misaligned with the respect to the second pore set that is going to be introduced. This means that the first pore set is not perpendicular to the etching direction of the second pore set. In 
Chapter 2. Fabrication and optical properties of inverse woodpile photonic crystals

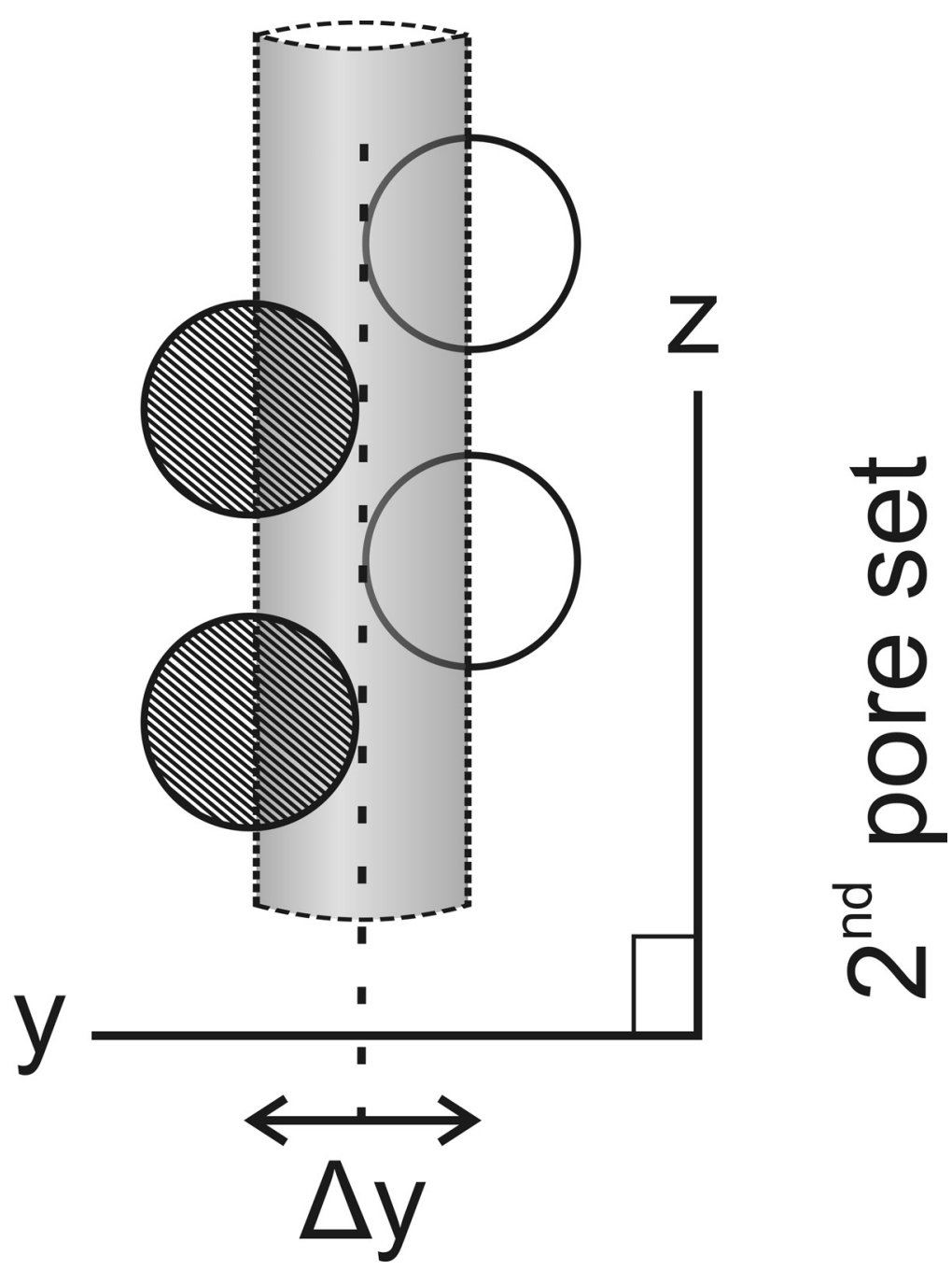

Figure 2.8: Representation of the $\Delta y$ displacement that may occur as a result of uncertainties in the processes by which the second set of pores are defined. The first set of pores points "into" the paper and the second set of pores runs parallel to the z-direction. The ideal position for pores in the second set is exactly in the middle of two rows of pores of the first set, indicated by the dashed line. Misalignment of the second set to the left or the right from its ideal position $(\Delta y)$ results in reduced relative photonic band gap widths. 


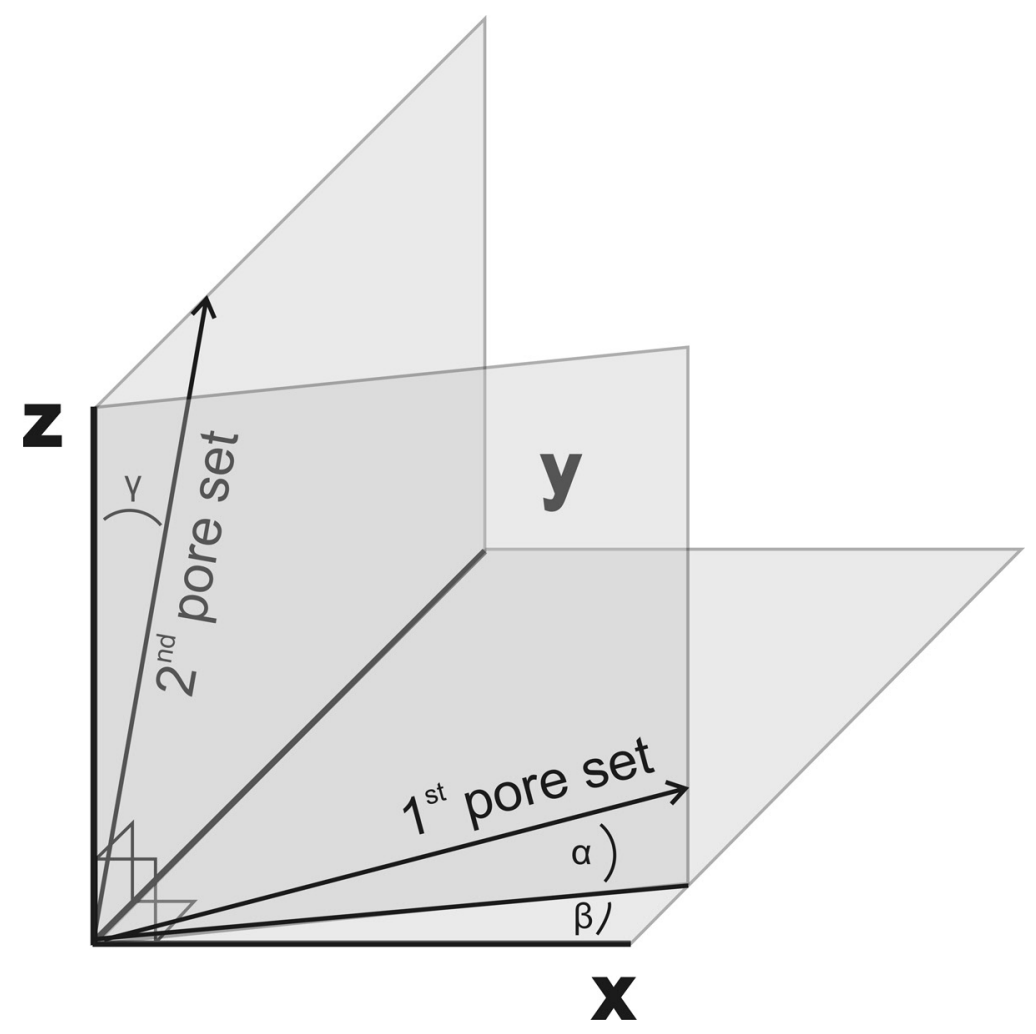

Figure 2.9: Schematic of possible angular misalignments that may occur during the fabrication of a three dimensional photonic structure. The $\mathrm{x}$-axis is the ideal direction of the first pore set and the z-axis of the second pore set. Angular misalignments are introduced when a sample with an existing set of pores is placed in the holder wafer. Misalignment by angle $\alpha$ occurs when the $x$-axis of the $x$ - $y$ surface of the samples is not mounted parallel to the wafer holder surface. Misalignment by angle $\beta$ occurs when the retrieved sample is mounted in the wafer holder with an in-plane rotational error. Misalignment by angle $\gamma$ occurs when a sample is mounted in the wafer holder with the $y$-axis of its $x$-y surface not parallel to the wafer holder surface. 
Chapter 2. Fabrication and optical properties of inverse woodpile photonic crystals

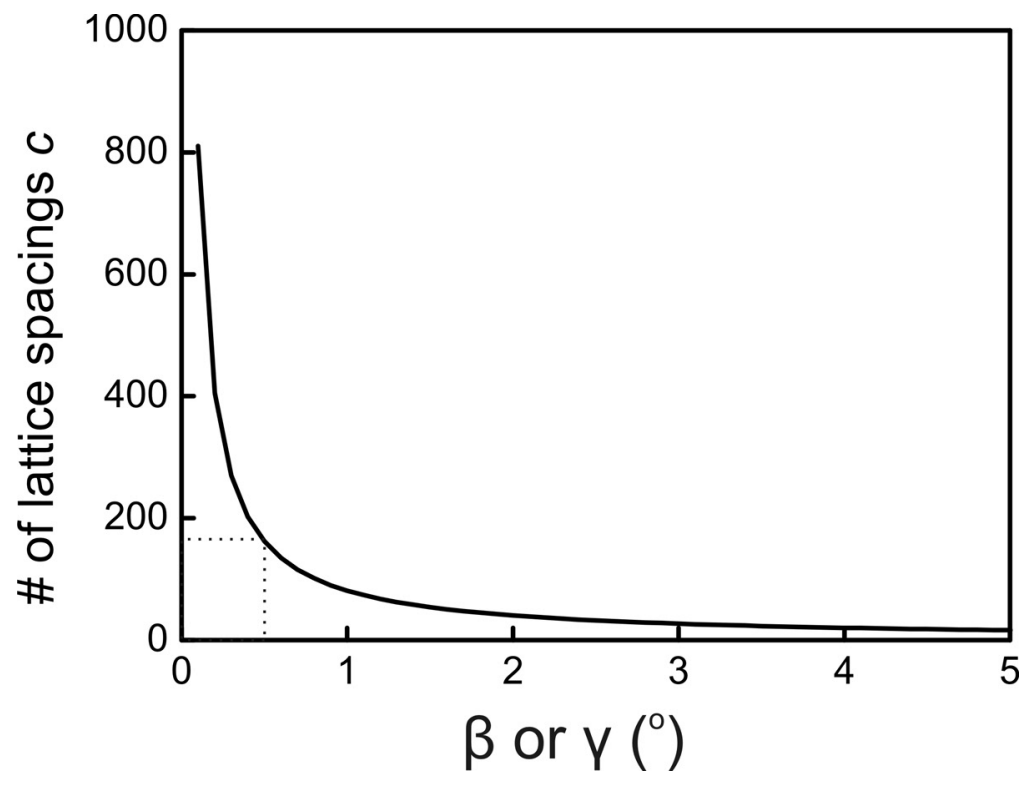

Figure 2.10: The number of lattice spacings $c$ that a pore crosses when it traverses one lattice spacing a due to angular misalignment versus the misalignment angle $\beta$ or $\gamma$. For $\beta$ or $\gamma=0.5^{\circ}$ the structure is periodic over 180 lattice spacings $c$. This value of $0.5^{\circ}$ will be taken by us as upper limit for these two misalignments individually.

other experiments where the second pore direction is milled using a focused ion beam setup without the use of a holder wafer, misalignment depends on how well the sample is positioned with respect to the ion beam. Figure 2.9 shows the three possible directions in which the two pore sets can be misaligned. The $\mathrm{x}$-axis is the ideal direction of the first pore set and the $\mathrm{z}$ axis the direction of the second pore set. The misalignment by angle $\gamma$ occurs when the retrieved sample as shown in Figure 2.2(C), is mounted with the $y$-axis tilting about the $\mathrm{x}$-axis out of the plane of the wafer holder surface. Misalignment by angle $\beta$ occurs when the retrieved sample is mounted in the wafer holder with an in-plane rotational error. Misalignment angle $\alpha$ occurs when the sample is mounted with the $\mathrm{x}$-axis tilting about the y-axis out of the plane of the wafer holder surface. Misalignment angles $\alpha$ and $\gamma$ can also be introduced in case the wafer holder is not properly mounted with respect to the etching direction of the second pore set.

Misalignment angles $\gamma$ and $\beta$ break the long-range periodic order of the three-dimensional structure. For this reason we can not determine the effect 
of these misalignments on the photonic band gap with the MIT photonic bands package, as band structures can only describe periodic structures ${ }^{2}$. Nonetheless we can illustrate to what extend these misalignments break the long-range periodic order of the structure by calculating after how many lattice spacings $c$ along the $\mathrm{x}$ - or z-direction, the structure deviates by one lattice spacing $a$, see Figure 2.10. With increasing misalignment angle, the pores need less lattice spacings $c$ to traverse one lattice spacing $a$, which indicates increasing non-crystallinity of the structure. For an angle $\beta$ or $\gamma<0.5^{\circ}$, the structure is periodic over $>180$ lattice spacings $c$. In our case this corresponds to $>86 \mu \mathrm{m}$. As we will see in chapter 4 , the pores that we can obtain by reactive ion etching have depths $h<10 \mu \mathrm{m}$, which means that in fabrication practice the calculated periodicity for misalignment angles $\gamma$ and $\beta<0.5^{\circ}$ can still be considered long-range. Since we lack a better method to quantify the effect of this misalignment, we choose the value of $0.5^{\circ}$ as upper limit for these two misalignments individually.

The situation where $\beta=\gamma=0^{\circ}$ and $\alpha \neq 0^{\circ}$ is discussed in detail in reference [128]. Increasing angle $\alpha$ results in a lower width of the band gap. The effect of an increasing misalignment that can be afforded is limited to an angle of about $5^{\circ}$ : a change from $(\Delta \omega / \omega)=24 \%$ at $\alpha=0^{\circ}$ to $(\Delta \omega / \omega)=21 \%$ at $\alpha=5^{\circ}$ is reported in [128], with a slightly increasing center frequency of the band gap. This result is similar to results described for a crystal of high refractive index woodpiles [129]. Since $\alpha$ is expected to remain far below $5^{\circ}$, the effect of this misalignment is negligible.

\subsubsection{Tapered pores}

During the fabrication of pores for a three-dimensional crystal by means of focused ion beam milling and reactive ion etching, it is common for the pores to be conically shaped. This tapering has a pronounced effect on the band gap of the crystal. Since in literature we did not find a detailed analysis of the effect of this deviation on the photonic band gap of a crystal, it will be further discussed in this section. Three situations may be distinguished: a) the crystal has one set of pores which displays tapering, whereas the other set is cylindrical,

b) both pore sets display the same amount of tapering, and

c) both pore sets are tapered, but with a different amount.

Here we will consider situation b) as it provides a general representation of

${ }^{2} \mathrm{~A}$ calculation is possible by using supercell input. However, the supercells that are needed for these calculations would be too large to be practically possible to use due to memory restrictions 
Chapter 2. Fabrication and optical properties of inverse woodpile photonic crystals

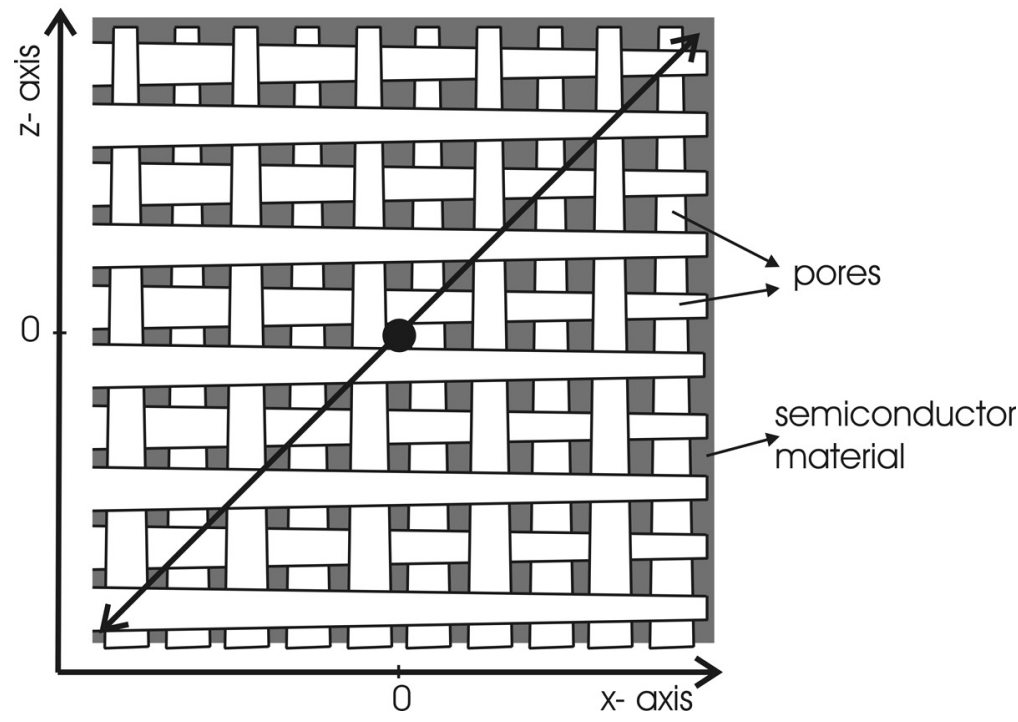

Figure 2.11: Schematic cross-section of a crystal of 10 pores by 10 pores with pores tapered in both directions. In the third dimension the crystal is assumed to be (infinitely) large. In the center of the crystal (black dot, $[\mathrm{x}, \mathrm{z}]=[0,0]$ ) the radii of both pore sets are equal to the desired radius. The diagonal shows positions in the crystal were $(R / a)$ is different, while the radii of both pore sets are equal. The arrows indicate the pores and the semiconductor material. The effect of the tapering on the local band gap is studied for positions along the diagonal. The crystal shown is approximately 4 times smaller than the fictitious crystal used for the calculations in this section.

the deviation and is the most straightforward case to analyze.

Figure 2.11 shows a crystal consisting of 10 pores by 10 pores with tapered pores in both directions. In the third dimension the crystal is assumed to be (infinitely) large, therefore we limit our discussion to the $\mathrm{x}-\mathrm{z}$ plane of the crystal. In the center of the crystal (black dot, $[\mathrm{x}, \mathrm{z}]=[0,0]$ ) the radii of both pores are equal to the desired radius. On any other position in the crystal the ratio $(R / a)$ of the two pore sets is different.

Along the diagonal from bottom left to top right (shown in the Figure) the radii of the two pore sets are equal, but different at all positions. This changes one quantity of the crystal: the ratio $(R / a)$ decreases, hence the volume fraction of silicon increases and thus the effective refractive index $n_{\text {eff }}$ is increased. Consequently, the width and the central frequency of the band gap are changing along this diagonal: the relative width of the band gap has an optimum at $[0,0]$, and the central frequency will decrease 
when going from bottom left to top right. Unfortunately it is not possible to calculate the band structure of a tapered crystal. However, with the following theoretical analysis, the effect of the tapering on the band gap can be estimated.

The fictitious photonic crystal under consideration has an ideal geometry at its center with $(R / a)=0.24$. There, the structure has a band gap at frequency $\omega_{\text {gap }}=0.564[\omega a / 2 \pi c]$. For non-tapered crystals with equal radii of both pore sets, we have calculated how the relative width of the band gap depends on $(R / a)$, see section 2.4.1. To estimate the effect of tapering on the band gap, the data in Figure 2.7 were used to calculate the upper and lower band edges of the "local photonic band gap" along the diagonal in crystals which have different amounts of tapering ${ }^{3}$. We calculated from -10 to $10 \mu \mathrm{m}$ along the $\mathrm{x}$ and the $\mathrm{z}$-axis of the structure, i.e., from [-10, $10]$ to $[10,10]$ (all coordinates are in units $[\mu \mathrm{m}, \mu \mathrm{m}]$ ).

Figure 2.12 shows the results of these calculations for four different amounts of tapering, namely $1,0.5,0.25$, and $0.125^{\circ}$. Each plot shows the upper and lower band edges versus position along the diagonal in the crystal. The horizontal dashed lines are at frequency $\omega_{\text {gap }}=0.564[\omega a / 2 \pi c]$, for which these crystals were optimized. The Figure shows that the photonic crystals are extremely sensitive to tapering of both pore sets. For tapering as little as $1^{\circ}$ the relative band gap width rapidly decreases when moving away from the center, see Figure 2.12(A). With decreasing tapering, the relative width of the band gap decreases less rapidly when moving away from $[0,0]$, see Figures 2.12(B) to (D).

As a measure of quality the transmittance $T$ along the diagonal is calculated for light with the frequency of the band gap center. Low transmittance is indicative of a strongly photonic behaviour and vice versa. Transmission $T$ depends on the Bragg attenuation length $L_{B}$ and can be calculated using equation 24 from reference [130]:

$$
T=\left[\cosh \left(\frac{h_{\text {bands }}}{L_{B}}\right)\right]^{-2} .
$$

In structures with no tapering $h_{\text {bands }}$ is equal to the thickness of the photonic crystal. In case of tapering, $h_{\text {bands }}$ is the length along the diagonal for which a band gap for the optimal frequency exists. In other words, as $h_{\text {bands }}$ gets longer, the volume of the crystal with a band gap increases. In

\footnotetext{
${ }^{3}$ The calculated data for the relative width of the band gap and the upper- and lower band edges in Figure 2.7 was reduced to $9^{t h}$ order polynomal functions which were then used to calculate the properties along the diagonal. The polynomal constants were determined with 8 digit precision.
} 
Chapter 2. Fabrication and optical properties of inverse woodpile photonic crystals

Figure $2.12 h_{\text {bands }}$ is determined by the points were the band gap edges cross the horizontal dashed line displaying the relative frequency for which these structures are optimized. Note that the real-space length of $h_{b a n d s}$ equals $\sqrt{(2)}$ times the length of $h_{\text {bands }}$ on the x-axis of the Figure.

In section 2.2 we introduced a perfect crystal with a lattice parameter $a$ of $680 \mathrm{~nm}$ and a radius $R$ of $163 \mathrm{~nm}$. In that case the photonic band gap has a relative width of $(\Delta \omega / \omega)=25.3 \%$ at frequency $\omega=8290 \mathrm{~cm}^{-1}$ $\left(\lambda_{\text {gap }}=1210 \mathrm{~nm}\right)$, which is detectable in our optical setups. For these crystals, the calculated Bragg attenuation length $L_{B}=1.52 \mu \mathrm{m}^{4}$. In the center of each plot in Figure 2.12 a horizontal bar equal to the calculated Bragg attenuation length $L_{B}$ is shown. In the direction of the x-z diagonal in Figure 2.11 this corresponds to 2.2 unit cells.

For a structure with $1^{\circ}$ tapering, $h_{\text {bands }}=2.1 \mu \mathrm{m}$. The calculated transmittance is as high as $T=0.22$, which indicates that the band gap has little effect on the transmission of light. For $0.5^{\circ}$ tapering $h_{\text {bands }}$ is limited to $h_{\text {bands }}=4.2 \mu \mathrm{m}$. Therefore only a small volume of the crystal has a photonic band gap. This results in a transmittance of $T=0.015$, which is almost 15 times better compared to $1^{\circ}$ tapering. Nonetheless, from the values calculated for $h_{\text {bands }}$ it is obvious that when the fabrication process yields pores with a tapering of 0.5 to $1^{\circ}$, it is not very useful to try to obtain pores deeper than a few micrometer, as pores deeper than $h_{b a n d s} / \sqrt{(2)}$ do not contribute to an effect of the photonic band gap. If we move on to the best case reported in Figure 2.12, we see that at a tapering of $0.125^{\circ}$ the transmittance is low at $T=1.2 \cdot 10^{-9}$, which shows that the band gap has a significant effect on the transmittance. In addition, $h_{b a n d s}=16.7 \mu \mathrm{m}$, which means that a photonic band gap exists in a whole $10 \times 10 \mu \mathrm{m}^{2}$ fictitious crystal. Here, trying to obtain pores deeper than a few micrometer is useful, as deeper pores contribute to stronger photonic crystals until a depth of $10 \mu \mathrm{m}$ is reached.

On all positions in the fictitious crystal which are not on the diagonal from bottom left to top right, the radii of the two pore sets are different. Unfortunately calculating the "local photonic band gap" properties for all these positions would be extremely time-consuming, which limits further exploration of the problem and limits an analysis of situations a) one pore set with tapering, and c) two pore sets with different amounts of tapering. However, the above analysis of the local gap along the $[-10,-10]$ to $[10,10]$ diagonal of the fictitious crystal, which is the worst-case in that crystal,

\footnotetext{
${ }^{4}$ In order to estimate an "effective" Bragg length $L_{B}$ for all three directions, the photonic strength $S$ in the direction of the diagonal is chosen to be equal to the relative width of the photonic band gap, therefore $\left(\Delta \omega_{\text {gap }} / \omega_{\text {gap }}\right)=S=25.3 \%$.
} 


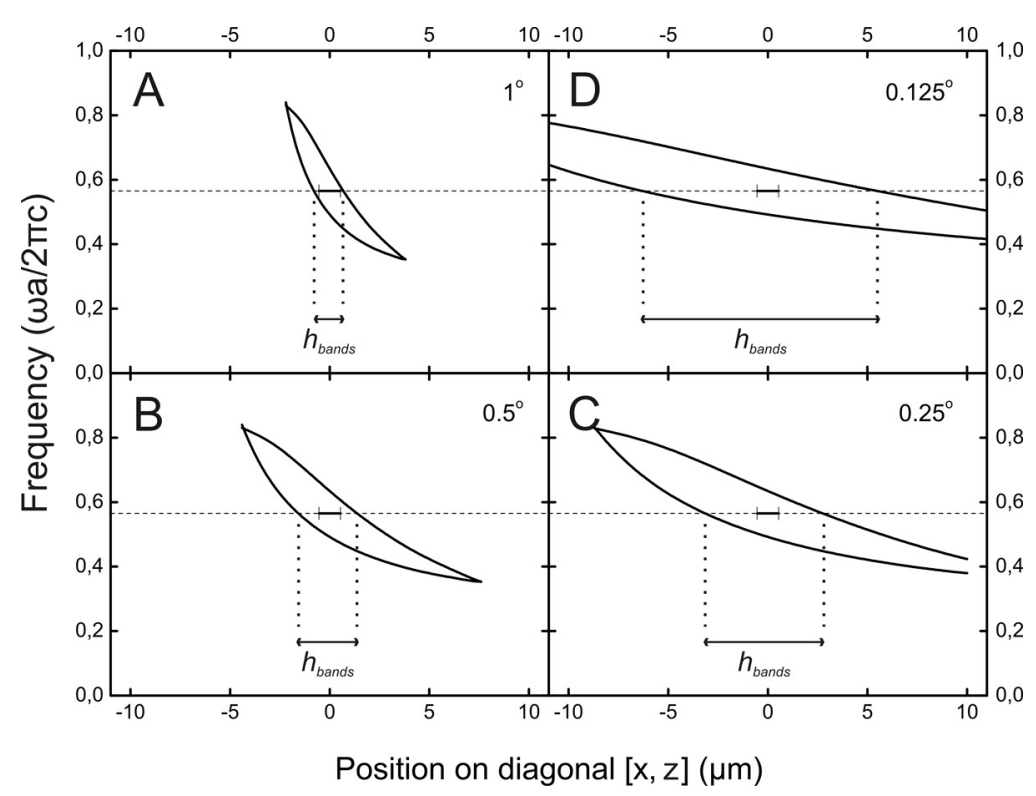

Figure 2.12: Estimated edges of the "local photonic band gap" at positions along the diagonal in Figure 2.11 for crystals with pores with different amounts of tapering. The horizontal dashed line displays the relative frequency for which these crystals are optimized. The horizontal bar in the center of each plot shows the Bragg length $\left.L_{B} . A\right), B$ ), and $C$ ) Results for a crystal with $1^{\circ}, 0.5^{\circ}$, and $0.25^{\circ}$ of tapering: the band edges are highly curved, and only a small part of these crystals exhibits a band gap as indicated by $h_{\text {bands }}$. D) At a tapering of $0.125^{\circ}, h_{\text {bands }}=16.7 \mu \mathrm{m}$, which spans more than half the length of the diagonal in the $10 \times 10 \mu \mathrm{m}^{2}$ fictitious crystal. The estimated transmittance is very low: $T=1.2 \cdot 10^{-9}$, which is indicative of a strongly photonic structure.

clearly indicates the importance of reducing the tapering as much as possible, preferably to zero.

\subsubsection{Different cylinder radii of the two sets of pores}

When two separate processes are used to obtain the two perpendicular pore sets, it is very challenging to obtain equal cylinder radii of the two sets of pores. This results in a difference in pore radii which is very difficult to minimize. This deviation was qualitatively discussed in reference [68] where it is mentioned that a maximum band gap is obtained when the two radii are equal. When the radii of the two sets are increasingly different, the relative width of the band gap decreases. Unfortunately reference [68] does 
Chapter 2. Fabrication and optical properties of inverse woodpile photonic crystals

not include any quantitative data and therefore we will look at this effect more closely.

Many scenarios can be distinguished how the two pore set radii differ, two of which will be discussed here:

a) the radius of the first pore set is according to specification $\left(\left(R_{1} / a\right)=0.24\right)$, but the radius of the second pore set is varied $\left(\left(R_{2} / a\right) \neq 0.24\right)$.

b) The first and second pore set both differ from ideal $(R / a)=0.24$, with $\left(R_{1} / a\right)$ larger and $\left(R_{2} / a\right)$ equally smaller than specified for a perfect structure.

With the MIT photonic bands package we have calculated the effects of these deviations on the relative width and central frequency of the band gap. By analyzing the calculated data, whilst choosing as a criterion that a photonic band gap width of $(\Delta \omega / \omega)>20 \%$ is acceptable, we can quantify the robustness of the crystal structure towards these geometrical deviations. In both scenarios lattice parameters $a$ and $c$ are constant.

a) Figure 2.13 shows the the band gap edges and the relative width of the band gap versus $\Delta(R / a)$. Difference $\Delta(R / a)$ is defined as:

$$
\Delta\left(\frac{R}{a}\right)=\left(\frac{R_{2}}{a}\right)-\left(\frac{R_{1}}{a}\right) .
$$

The central frequency of the band gap is lowest at small $\Delta(R / a)$, and increases with increasing $\Delta(R / a)$. A maximum relative width of the band gap is found for $\Delta(R / a)=0$. The relative width of the band gap decreases as $\Delta(R / a)$ differs from zero. The band gap is most sensitive to an increasing ratio $\left(R_{2} / a\right)$. When $\Delta(R / a)=+0.048$, the relative width of the band gap decreases to less than $5 \%$. In contrast, when $\Delta(R / a)=-0.048$, the relative band gap width remains $15 \%$. We conclude that our criterion of a minimal relative width of the band gap of $(\Delta \omega / \omega)=20 \%$ is found for $\Delta(R / a) \leq \pm 0.024$. This corresponds to a large difference in the etched radius $\Delta R \leq \pm 16 \mathrm{~nm}$. Typically the achieved radius will be much closer to the target radius.

b) Figure 2.14 shows the the band gap edges and the relative width of the band gap versus $\Delta\left(R_{(1+2)} / a\right)$. The difference in ratios $\Delta\left(R_{(1+2)} / a\right)$ is defined as:

$$
\Delta\left(\frac{R_{(1+2)}}{a}\right)=\left(\frac{R_{1}}{a}\right)-0.24 \text { and } \Delta\left(\frac{R_{(1+2)}}{a}\right)=0.24-\left(\frac{R_{2}}{a}\right) .
$$

The relative width of the band gap decreases as $\Delta\left(R_{(1+2)} / a\right)$ differs from zero. When the pore radii are changed by $\Delta\left(R_{(1+2)} / a\right)=+0.035$, the relative 


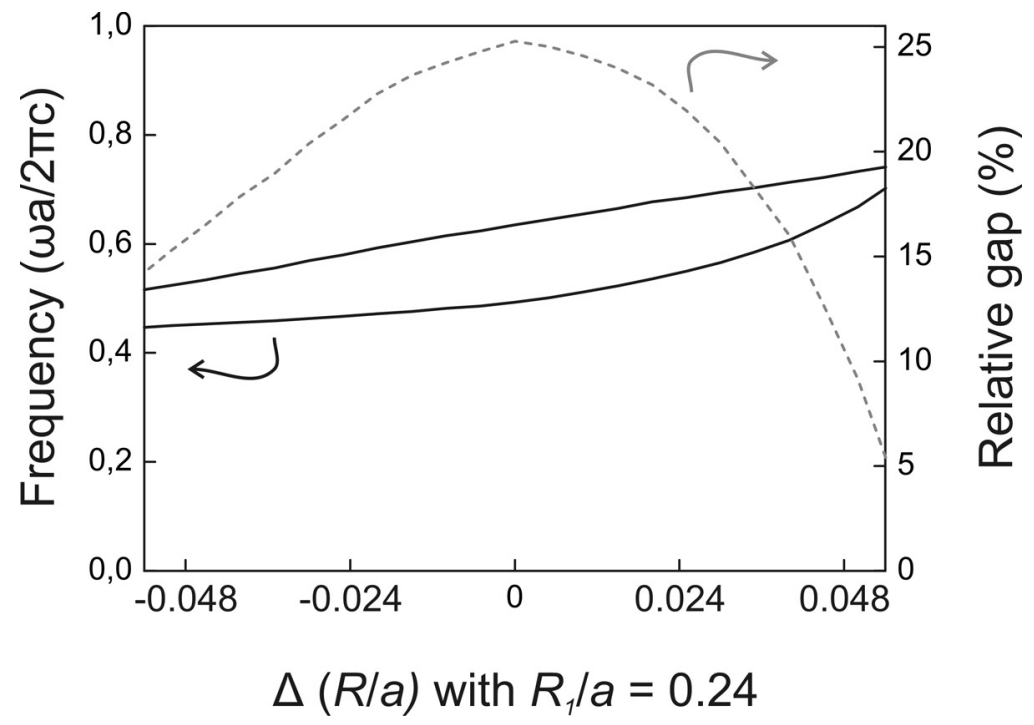

Figure 2.13: Calculated relative width of the band gap (dashed line) and band gap edges (solid lines) plotted against $\Delta(R / a)$ for crystals with $\left(R_{1} / a\right)=0.24$ and $\left(R_{2} / a\right)$ varied from 0.18 to 0.30 . The relative width of the band gap decreases as the difference between $\left(R_{2} / a\right)$ and $\left(R_{1} / a\right)$ gets larger. If $\Delta(R / a)$ is changed by \pm 0.024 , a relative width of the photonic band gap of more than $(\Delta \omega / \omega)=20 \%$ remains. When $\Delta(R / a)=+0.048$, the relative width of the band gap is decreased to less than $30 \%$ of the maximum value. For $\Delta(R / a)=-0.048$, around $60 \%$ of the maximum relative width of the band gap remains. The band gap edges are lowest at small $\Delta(R / a)$, and increase as $\Delta(R / a)$ increases.

width of the band gap is halved. For $\Delta\left(R_{(1+2)} / a\right)=+0.05$, the band gap vanishes. We conclude that our criterion of a minimal relative width of the band gap of $(\Delta \omega / \omega)=20 \%$ is found for $\Delta\left(R_{(1+2)} / a\right) \leq 0.018$, which corresponds to large difference in the etched radius of around $R \leq 12 \mathrm{~nm}$. However, the achieved radius will typically be much closer to the target radius. In addition, Figure 2.14 shows that the central frequency of the band gap remains at almost the same position as $\Delta\left(R_{(1+2)} / a\right)$ increases, which is convenient: even when this deviation occurs during fabrication of the crystal, it will nonetheless be active at the optical frequency it was designed for.

From a) and b) we conclude that inverse woodpile photonic crystals are robust to differences between optimized $\left(R_{1} / a\right)$ and $\left(R_{2} / a\right)$ that may occur as a result of the fabrication process. Furthermore, the tolerable fabrication margins are expected to remain well within our capabilities, therefore good 
Chapter 2. Fabrication and optical properties of inverse woodpile photonic crystals

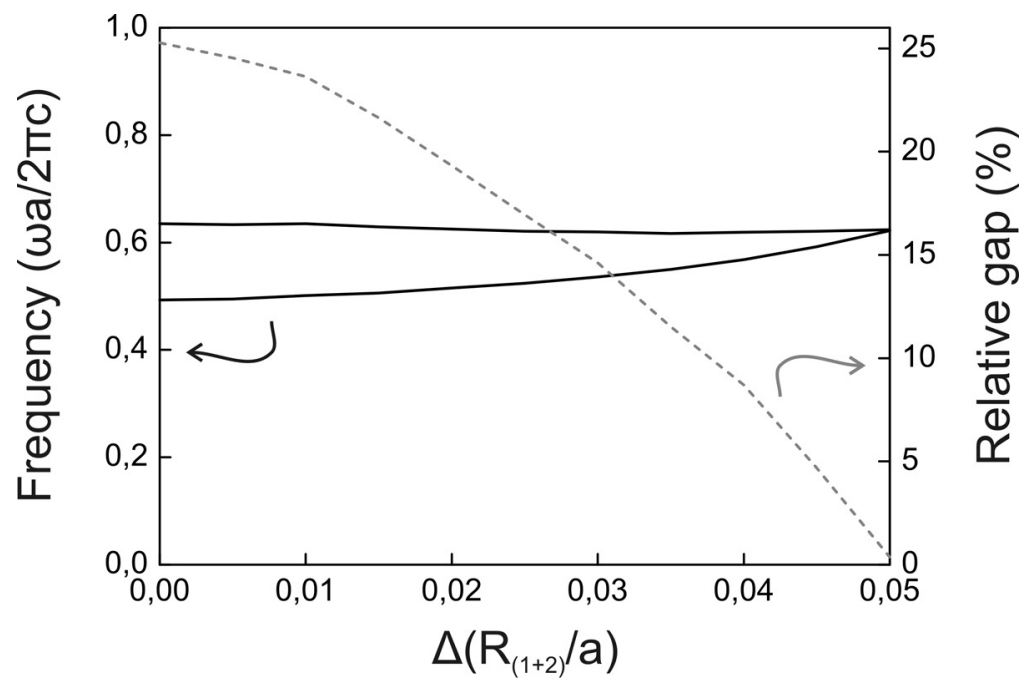

Figure 2.14: Calculated relative width of the band gap (dashed line) and band gap edges (solid lines) for crystals with both $\left(R_{1} / a\right)$ and $\left(R_{2} / a\right)$ varying by equal values from $(R / a)=0.24$. Ratio $\left(R_{1} / a\right)$ is increased, whereas $\left(R_{2} / a\right)$ is decreased. Data is shown for $\Delta\left(R_{(1+2)} / a\right)$ up to 0.05 , calculated in 0.005 increments. The relative width of the band gap decreases as increases. At a difference of $\Delta\left(R_{(1+2)} / a\right)=0.035$, the relative width of the band gap is halved. At a difference of $\Delta\left(R_{(1+2)} / a\right)=0.05$ the band gap has vanished. The center of the two band gap edges slightly increases as $\Delta\left(R_{(1+2)} / a\right)$ increases.

quality crystals may be expected.

\subsection{Optimized broad photonic band gap}

In reference [70] an inverted woodpile photonic crystal is described, fabricated from ellipsoidal pores. There, a larger band gap is predicted when the ratio $(a / c)$ in their structure is increased from $\sqrt{2}$ to 1.63 . Motivated by this observation, we wanted to investigate whether the ratio $(a / c)=\sqrt{2}$ that we aim for in our fabrication is optimal for photonic crystals.

Using the MIT photonic bands package we calculated the relative width of the band gap for structures with varying ratios of $(a / c)$. Since the optimal $(R / a)$ also varies with the chosen $(a / c)$, we also varied $(R / a)$ in our calculations. The ratio $(a / c)$ was varied from 1 to 1.8 , with increments as small as $|\Delta(a / c)|=0.025$ in the region of the maximum relative width of the band gap. The ratio $(R / a)$ was varied from 0.18 to 0.28 . with increments of 


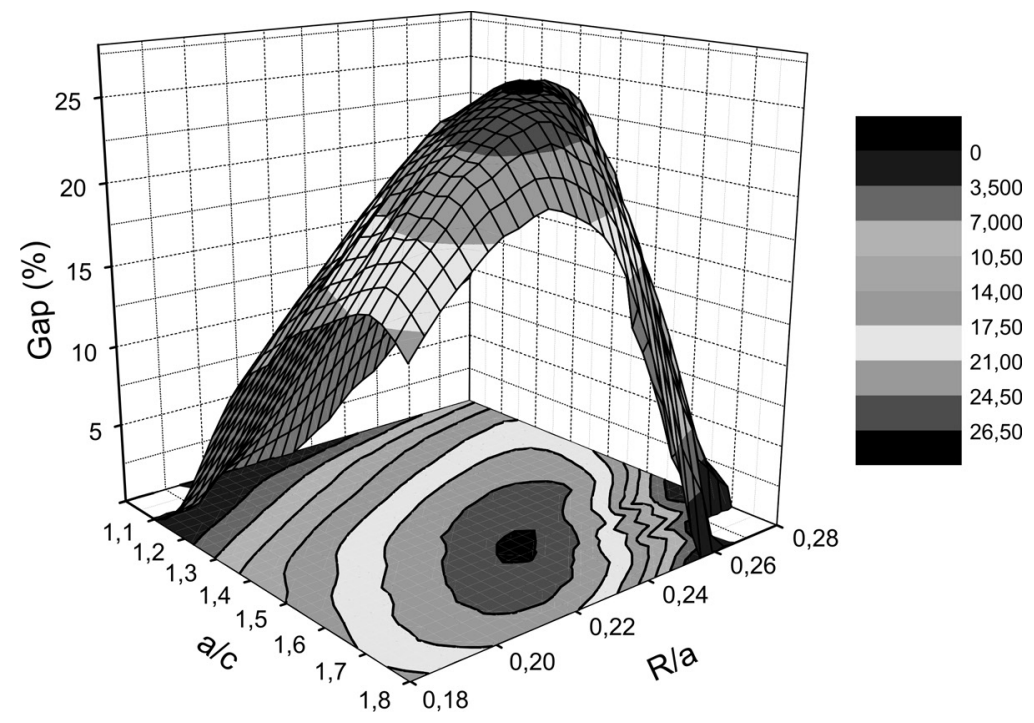

Figure 2.15: Interpolated three-dimensional surface- and contour plot of the calculated band gaps versus the ratios $(R / a)$ and $(a / c)$. The Figure shows that there is a large region of $(a / c)$ and $(R / a)$ combinations where the relative width of the band gap is much larger than $(\Delta \omega / \omega)=26.5 \%$, see the black circular area around $(a / c)=1.6$ and $(R / a)=0.23$. The broadest photonic band gap of $(\Delta \omega / \omega)=26.9 \%$ is found at $(a / c)=1.575$ and $(R / a)=0.23$. The Figure also shows that when a three-dimensional crystal is fabricated from two sets of hexagonal cylindrical pores with high symmetry $((a / c)=1)$, there is only a very small band gap found.

$|\Delta(R / a)|=0.0025$ in the region of the maximum relative width of the band gap.

The largest band gap is found for $(a / c)=1.575$ and $(R / a)=0.23$. At these values, a relative width of the band gap of $(\Delta \omega / \omega)=26.9 \%$ is calculated $^{5}$, slightly larger than the band gap of $(\Delta \omega / \omega)=25.3 \%$ for the structure with $(a / c)=\sqrt{2}$ and $(R / a)=0.24$ that we hitherto referred to as perfect (cubic) crystal. The increase in relative band gap width is more than $\Delta(\Delta \omega / \omega)=1.6 \%$, and in the future can be easily obtained by straightforward modification of the lithographic processes in the fabrication route of the inverse woodpile photonic crystals.

Figure 2.15 shows an interpolated three-dimensional surface- and contour plot of the calculated band gaps versus the ratios $(R / a)$ and $(a / c)$. The plot was interpolated using the Renka-Cline gridding method in Origin 7 . Figure 2.15 shows that there is a large region of $(a / c)$ and $(R / a)$ combinations

\footnotetext{
${ }^{5}$ This result is equal to results reported in reference [131]
} 
Chapter 2. Fabrication and optical properties of inverse woodpile photonic crystals

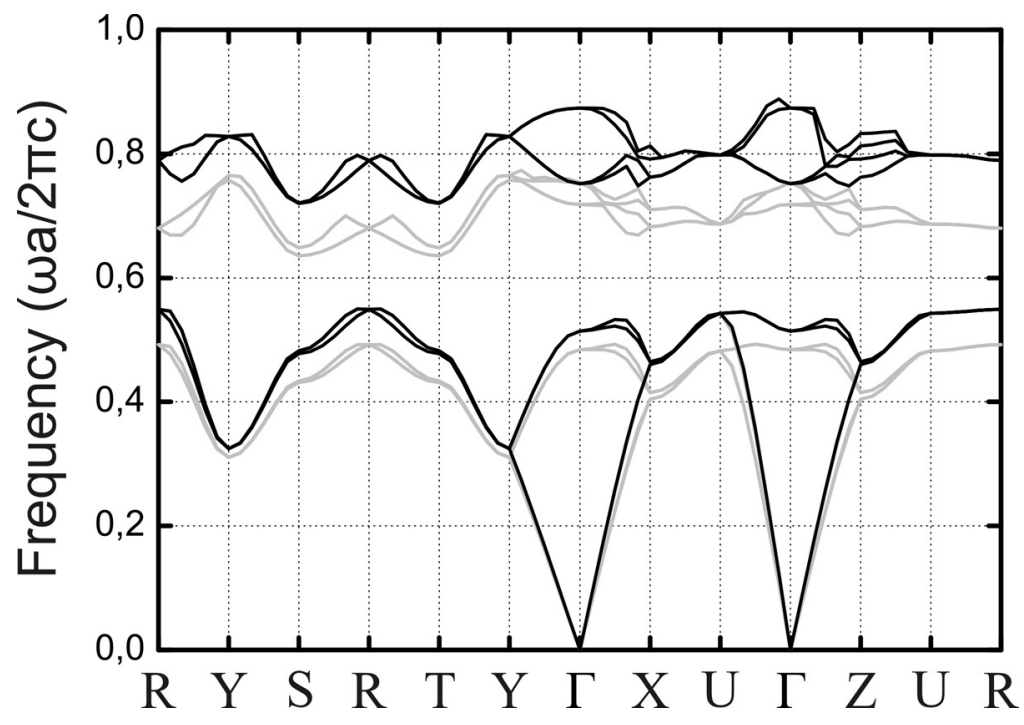

Figure 2.16: Calculated band structures of the optimal structure with $(a / c)=1.575$ and $(R / a)=0.23$, and the calculated band structures of the crystal with $(a / c)=\sqrt{2}$ and $(R / a)=0.24$. The central frequency of the optimal structure is $\omega=0.635[\omega a / 2 \pi c]$, compared to $\omega=0.564[\omega a / 2 \pi c]$ for the other structure. Apart from the height on the $y$-axis, the lower bands of the two crystals are equal. For most vectors in the Brillouin zone, the upper bands are also equal, with the exception of the $\Gamma-Y, \Gamma-X, \Gamma-U$ and $\Gamma-Z$ directions.

where the relative band gap width is much larger than $(\Delta \omega / \omega)=26.5 \%$, see the black circular area around $(a / c)=1.6$ and $(R / a)=0.23$. Naively we expected an optimal result for the case where two hexagonal patterns of pores are combined ( $(a / c=1)$, due to the high symmetry of the hexagonal lattice. However, Figure 2.15 shows that only a very small band gap is found for $(a / c)=1$.

Figure 2.16 shows the calculated band structures of the optimal structure with $(a / c)=1.575$ and $(R / a)=0.23$, and the calculated band structures of the crystal with $(a / c)=\sqrt{2}$ and $(R / a)=0.24$. For the optimal inverse woodpile photonic crystal, the frequencies of the band edges are higher. The central frequency of this structure is $\omega=0.635[\omega a / 2 \pi c]$, compared to $\omega=0.564[\omega a / 2 \pi c]$ for the cubic structure. This difference is due to the decreased volume fraction of silicon in the structure which results in a lower effective refractive index $n_{\text {eff }}$. When comparing the band structures of both types of crystal, we observe that the lower 4 bands are scaled along the frequency axis because of the different effective refractive indices. For most 
wave-vectors in the Brillouin zone, the upper bands are also scaled, with the exception of the $\Gamma-Y, \Gamma-X, \Gamma-U$ and $\Gamma-Z$ directions, which are clearly different. Apparently these are the directions that are most influenced by the changing geometry of the structure.

\subsection{Conclusions}

In this chapter several routes and fabrication techniques were discussed to manufacture inverse woodpile three-dimensional photonic crystals. A detailed scheme was described for a fabrication route which has the potential to produce crystals with large volumes. This route consists of etching of a first set of pores using photo electrochemical etching, followed by tilting and mounting the sample in a holder wafer with high precision. Then a second set of pores is accurately etched perpendicularly and well-aligned to the first set using a reactive ion etching process. These fabrication techniques are compatible with methods used in the complementary metal oxide semiconductor (CMOS) industry. We have also discussed possible unintended deviations from a perfect structure that may be introduced by the fabrication process. Although in general the band gap of this type of crystal is robust to disorder, we find that these crystals are very sensitive to tapering of the pores. Nonetheless, our analysis shows that for tapering values that we are able to obtain during fabrication, these crystals are still strongly photonic. By closely looking at the lattice parameters of the cubic inverse woodpile photonic crystal, we calculated that an alternative geometry potentially provides a slightly larger photonic band gap. In the future, our lithographic processes could be changed to pursue the fabrication of this geometry. 

Chapter 3

\section{Photo electrochemical etching of pores in silicon}

\subsection{Introduction}

\subsubsection{Photo electrochemical etching}

Electrochemical etching is a powerful tool for the fabrication of porous silicon. This technology has been used to manufacture numerous devices, such as capacitors [132], gas sensors [133], and microchannels for total analysis systems [134]. Electrochemical anodic etching is of particular interest in obtaining periodic arrays of pores in silicon for the fabrication of photonic crystals [15]. Many different varieties of the etching process exist [135, 136], but notably, to obtain photonic crystals, photo electrochemical etching on n-type wafers has been applied successfully. This was demonstrated in a number of inspiring examples [51, 137-139]. Therefore this is the technique that we will study in this chapter. One of the most essential advantages of choosing this fabrication method is that it allows very deep pores to be etched with smooth sidewalls, as is convincingly demonstrated in [121]. Therefore, photo electrochemical etching is suited as the first etch step in the fabrication scheme discussed in chapter 2. Etched pores can have a large range of diameters, from $<10 \mathrm{~nm}$ to many $\mu \mathrm{m}$ 's. For photonic crystals with band gaps in the telecommunication windows, the periodic arrays need to consist of pores with diameters below $500 \mathrm{~nm}$ and interpore distances below $1000 \mathrm{~nm}$.

The photo electrochemical etching of n-type silicon wafers is excellently described in [135] and [136]. Here a more concise explanation is given. Figure 3.1 is a schematic of how such an etching process proceeds. The fabrication of the pores is started by patterning of the wafer with periodic arrays of pore tips with the desired distance between them. The position of these pore tips defines where the actual pores will be etched. Indicated in the 


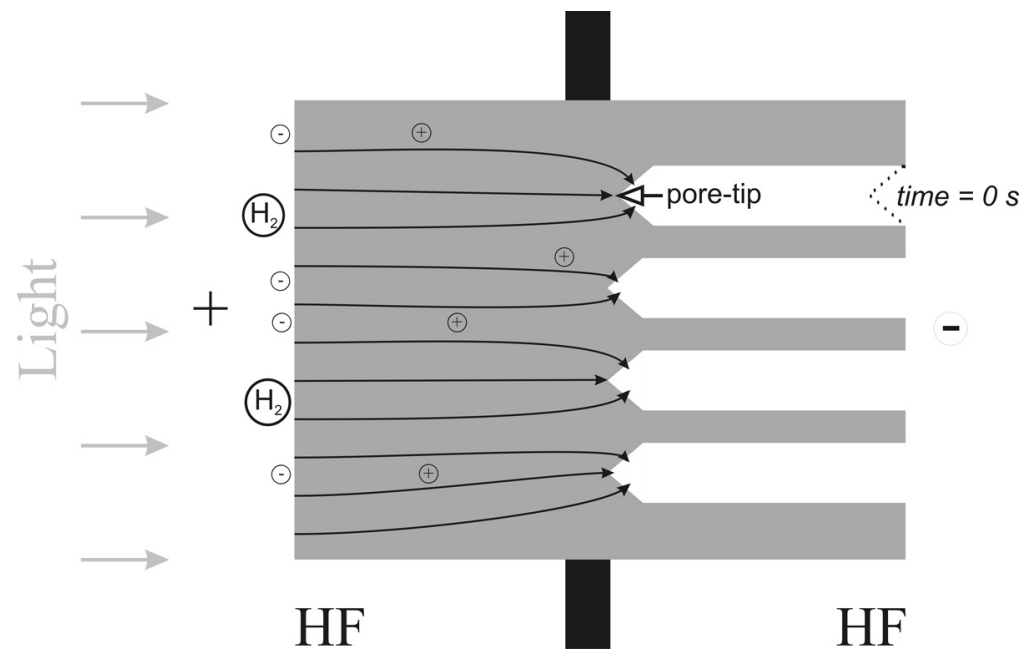

Figure 3.1: Schematic of the photo electrochemical etching process. In the upper right section, the dotted tilted pyramid shows a pore tip at the surface of the wafer before etching. The open arrow in the upper section of the Figure indicates a pore tip after etching for some time. A potential over the wafer generates an electric field of which the field lines selectively point towards the pore tips, indicated by the black arrows. Upon illumination of the backside of the wafer with light of wavelengths $\lambda$ $<1100 \mathrm{~nm}$, electron-hole pairs are generated. As the holes follow the electric field, they move to the pore tips and the silicon is etched. This results in deeply etched pores. During the chemical reaction, $\mathrm{H}_{2}$ is generated at the backside of the wafer. Figure courtesy of R.W. Tjerkstra.

upper right section of the Figure is a pore tip. The dotted tilted pyramid shows the pore tip at the surface of the wafer before etching. The open arrow points towards a pore tip after etching for some time. By applying a potential over the wafer, an electric field is generated of which the field lines selectively point towards the pore tips, indicated by the black arrows in the Figure. Upon illumination of the backside of the wafer with photons that have wavelengths $\lambda<1100 \mathrm{~nm}$, electron-hole pairs are generated because the energy of such photons exceeds the electronic band gap of silicon $(\sim 1.1 \mathrm{eV})$ [135]. Due to the electric field, the holes and electrons are separated and as the holes follow the electric field, they move to the pore tips. The electrons are consumed in a chemical reaction which generates $\mathrm{H}_{2}$ at the backside of the wafer. At the pore tips the combination of reactive fluor species and the holes allows for the silicon to be dissolved in the aqueous electrolyte. When all process parameters have been correctly chosen, etching will result in periodic arrays of deep pores, suitable for photonic crystal applications. 
Choosing the correct process parameters is a challenge, as they are different for dissimilar etching setups. This means that experimental details as outlined in articles in literature can not be easily adapted. Furthermore, most relevant articles do unfortunately not provide a detailed experimental description, which further necessitates careful screening of the process parameters.

\subsubsection{Etching parameters}

If we take a closer look at how to run an etching experiment, we identify a number of important experimental parameters that we will explain in more detail. These parameters are the potential difference $U$ that generates the electric field, the applied current $I$ or current density $J$, the intensity of backside illumination $L$, and the resistivity of the wafer.

To identify the correct etching parameters, $I-U$ curves are measured of the wafer that is going to be etched, see Figure 3.2. These curves are measured under $100 \%$ illumination such that there are always enough electron-hole pairs present during the dissolution of silicon. By scanning the potential $U$ and measuring current $I$, the characteristical trajectory depicted by the black line in Figure 3.2 is obtained. The first peak indicates the critical current $I_{p s}$. This is the highest current at which pores can be obtained in the wafer. At currents exceeding $I_{p s}$, electropolishing occurs, and no pores are formed [120]. Decreasing the illumination intensity decreases the amount of holes available for the etching process, and therefore the current $I$ decreases at constant potential. In this fashion, the entire area below the black line in Figure 3.2 is accessible by setting a potential $U$ and adjusting the desired etch current $I$ through the light intensity $L$ on the wafer. The hatched area marks the region where pore formation occurs.

The ratio of current $I$ to critical current $I_{p s}$ directly relates to the diameter of the etched pores $D_{\text {pore }}[140,141]$ :

$$
D_{\text {pore }}=a \sqrt{\frac{4}{\pi} \cdot \frac{I}{I_{p s}}}=a \sqrt{\frac{4}{\pi} \cdot \frac{J}{J_{p s}}},
$$

with $a$ the lattice parameter, or interpore distance, of a square lattice. By dividing current $I$ with the exposed surface area of the wafer $A$, the current density $J$ is obtained:

$$
J=\frac{I}{A}
$$




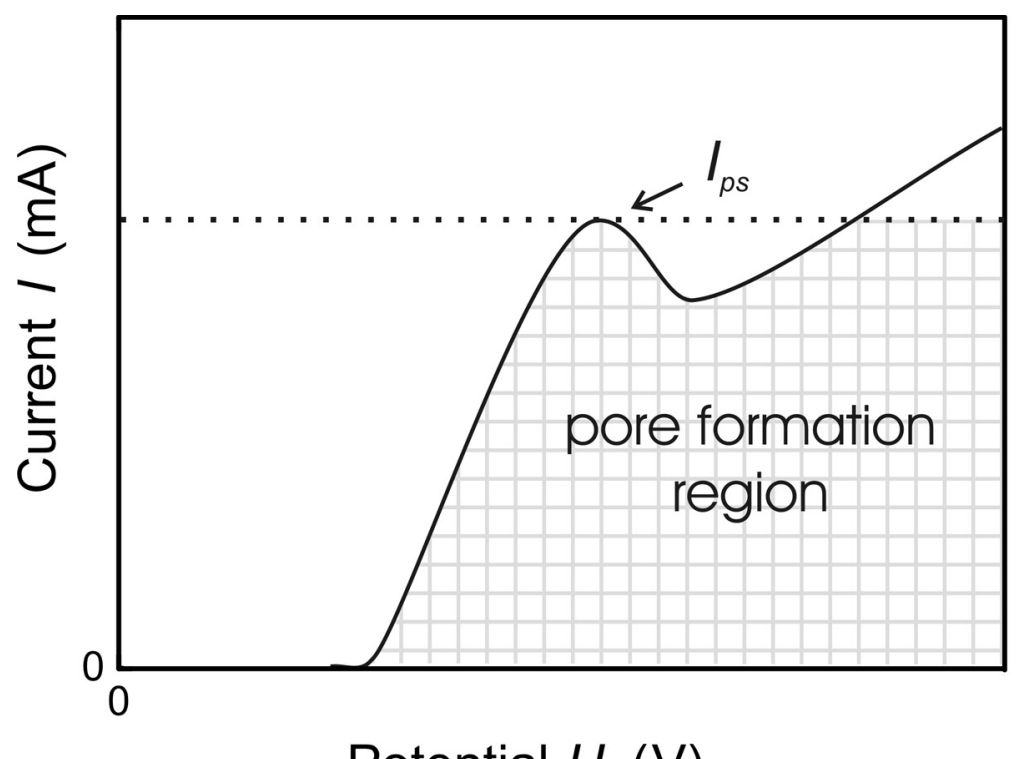

\section{Potential U (V)}

Figure 3.2: Schematic impression of a $I-U$ curve of an n-type silicon wafer. The black line shows the current I versus potential $U$ under $100 \%$ illumination. $B y$ reducing the illumination intensity, the current can be reduced below this black line. At the top of the peak, the arrow and the horizontal dotted line indicate the critical current $I_{p s}$. When etching with currents less than $I_{p s}$, pore growth occurs. However, when the current exceeds $I_{p s}$, the wafer is electropolished. By changing the potential $U$, the pore density can be changed when etching, or in our case, the etching process can be matched to the pre-defined lattice parameter a of the pore tips. The hatched area marks the region where pore formation occurs. However, at too high potentials branching occurs.

$J_{p s}$ is the critical current density. From this point on we prefer to talk about current density $J$, but for some of the calculations reported in this chapter it is unavoidable that we use the current $I$.

To obtain pores with the desired diameter, the correct current density must be chosen during etching, see Figure 3.3 and equation 3.1. Furthermore, the current density must be kept constant during the entire etching process ${ }^{1}$. Setting the wrong current density results in pores with other diameters than intended. Failure to properly regulate the current density may result in pores that have unwanted varying diameters at different depths. Some groups have

\footnotetext{
${ }^{1}$ For very deep pores it may be necessary to adjust current density $J$ in order to maintain the same pore diameter. This is due to diffusion of molecules out of the pores being more difficult and the diffusion of holes to the pore tips more easy $[136,140]$.
} 


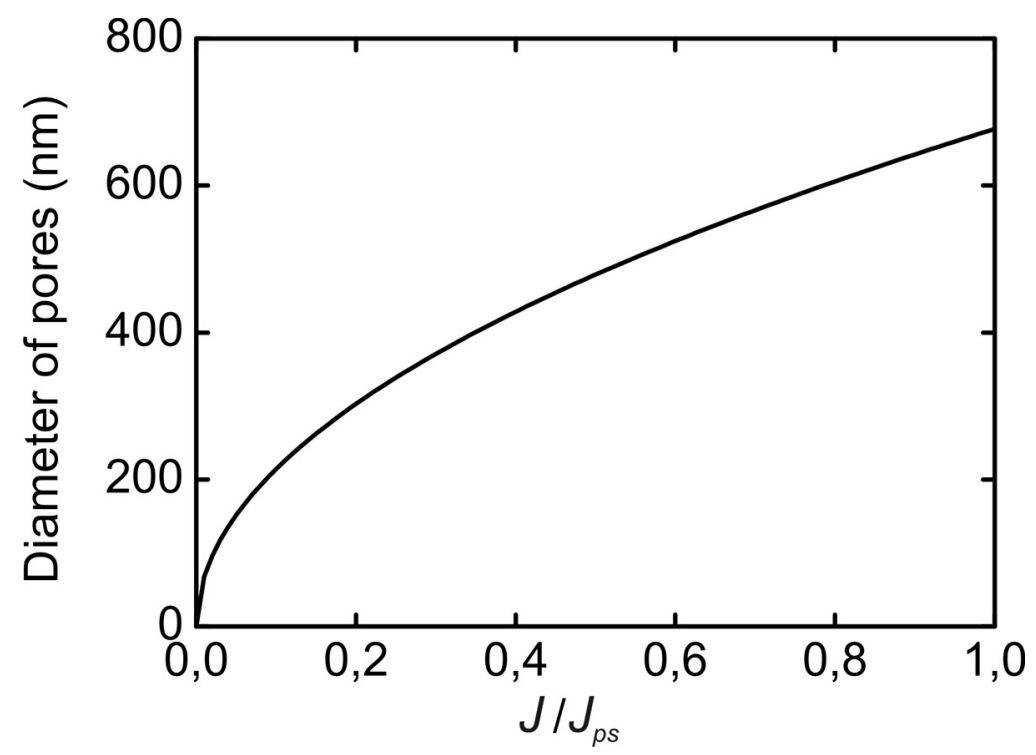

Figure 3.3: Calculated diameter of the etched pores as a function of the ratio of etch current density $J$ to critical current density $J_{p s}$, derived for a square lattice (equation 3.1). In this case, the lattice parameter of the pre-defined pore tips is $a=600 \mathrm{~nm}$. To obtain pore diameters of $300 \mathrm{~nm}$, the etch current needs to be reduced to approximately $20 \%$ of $J_{p s}$.

managed to exploit the relation between current density and pore diameter to fabricate three-dimensional crystals of regularly modulated pores $[142,143]$.

If we turn our attention to the potential $U$ during etching, we find that a correct setting must be chosen. If the potential is set higher than optimal, branching of the etched pores occurs [120]. This means that besides the main pores which are etched vertically, many smaller pores grow outward from the main pores, resulting in unusable structures. At low potentials, pore formation does not occur.

Choosing wafers with the correct resistivity is another essential aspect to obtain pores with the desired diameters and interpore distances. The resistivity is determined by the dopant concentration in the n-type wafer [144]. The lower the dopant concentration, the higher the wafer resistivity. Choosing the wrong wafer resistivity may result in pore growth that does not match the interpore distance of the array of pores [120]. If the resistivity is too high, the pores will tend to grow too far apart from each other. This will result in a phenomenon called "joining". In the case of a too low resistivity, the pores grow closer to each other than pre-defined. Although pore growth 
starts at the pore tips, at some point the pores will split into sub-pores. This is called "dividing". In reference [145] it is shown that the relation between etched pore diameter and substrate resistivity is linear on a log-log scale.

\subsubsection{Chemical reactions}

The photo electrochemical etching process breaks apart into three different regimes. The (overall) chemical reactions of these three regimes are generally accepted to read as follows [146, 147]:

Regime a: the etching current density is lower than the critical current density $\left(J<J_{p s}\right)$. In this situation pore formation occurs. Since our electrolyte contains ethanol, this implies that the HF in the electrolyte does not dissociate in fluoride species like $\mathrm{F}^{-}$and $\mathrm{HF}_{2}^{-}$. Thus the concentration of $\mathrm{HF}$ or its dimer $\mathrm{H}_{2} \mathrm{~F}_{2}$ is $>99 \%$ of the total concentration of fluoride species. Silicon is dissolved via the overall reaction

$$
S i_{(s)}+6 H F_{(a q)}+2 h^{+} \rightarrow S i F_{6(a q)}^{2-}+H_{2(g)}+4 H_{(a q)}^{+} .
$$

In this case $\mathrm{H}_{2}$ evolves from the electrolyte.

Regime b: at the peak at current density $J_{p s}$ a transition between a pure silicon- and a silicon oxide wafer surface is encountered. The silicon is dissolved via a silicon dioxide intermediate. First the silicon is oxidized by water:

$$
\mathrm{Si}_{(s)}+2 \mathrm{H}_{2} \mathrm{O}+2 h^{+} \rightarrow \mathrm{SiO}_{2(s)}+\mathrm{H}_{2(g)}+2 \mathrm{H}_{(a q)}^{+} .
$$

Subsequently the silicon dioxide is removed by the HF:

$$
\mathrm{SiO}_{2(s)}+6 \mathrm{HF}_{(a q)} \rightarrow \mathrm{SiF}_{6(a q)}^{2-}+2 \mathrm{H}_{2} \mathrm{O}+2 \mathrm{H}_{(a q)}^{+} .
$$

The overall reaction reads:

$$
S i_{(s)}+6 H F_{(a q)}+2 h^{+} \rightarrow S i F_{6(a q)}^{2-}+H_{2(g)}+4 H_{(a q)}^{+} .
$$

with $\mathrm{H}_{2}$ evolving from the electrolyte, similar to reaction 3.3 .

Regime c: the etching current density exceeds the critical current density ( $J$ $\left.>J_{p s}\right)$. In this case electropolishing occurs. The complete wafer surface is covered with oxide. Here also, the silicon is first oxidized by water according to the reaction

$$
S i_{(s)}+2 \mathrm{H}_{2} \mathrm{O}+4 h^{+} \rightarrow \mathrm{SiO}_{2(s)}+4 H_{(a q)}^{+} .
$$


Subsequently the silicon dioxide is removed by the HF:

$$
\mathrm{SiO}_{2(s)}+6 \mathrm{HF}_{(a q)} \rightarrow \mathrm{SiF}_{6(a q)}^{2-}+2 \mathrm{H}_{2} \mathrm{O}+2 \mathrm{H}_{(a q)}^{+} .
$$

The overall reaction reads:

$$
S i_{(s)}+6 H F_{(a q)}+4 h^{+} \rightarrow S i F_{6(a q)}^{2-}+6 H_{(a q)}^{+} .
$$

During this reaction, no $\mathrm{H}_{2}$ is generated.

\subsection{Etching setup}

The photo electrochemical etching setup we built for the experiments is shown schematically in Figure 3.4. Etching was performed in a Porous Silicon System PSB 4, purchased from AMMT GmbH (Germany). The PSB 4 is an etch bath made from polypropylene. It consists of two compartments, electrically separated by an insulating wall. Wafers with a diameter of $100 \mathrm{~mm}$ are mounted in an opening in this wall between the two chambers of the etch bath, using wafer holders. We used two types of wafer holders: 1) a so called "full wafer holder", which allows exposure of $A=58.1 \mathrm{~cm}^{2}$ of the wafer surface, and 2) a "test wafer holder", which allows smaller areas of $A=3.8 \mathrm{~cm}^{2}$ of the wafer to be etched. With the "test wafer holder" each wafer can be used in four tests, which saves on wafer consumption. By swapping covers, the wafer holders are modified to accept wafers with different thicknesses. The etch bath is filled with 5 liters of electrolyte. Drain valves allow for the easy removal of electrolyte from the bath. On both sides of the bath sapphire windows, protected by fused silica disks, allow illumination or viewing of the wafer. The window not in use is covered to prevent illumination of the frontside of the wafer.

Typically the etched wafers were illuminated by a $75 \mathrm{~W}, 230 \mathrm{~V}$ Sylvania Hi-SPOT $\left(10^{\circ}\right)$ halogen lightbulb. The light was focused on the wafers by a Melles-Griot plano-convex uncoated lens with a diameter of $130 \mathrm{~mm}$ and a focal distance of $200 \mathrm{~mm}$. The lens was placed as close as possible to the etch bath, which is approximately $5 \mathrm{~cm}$ from the window. The lightbulb was placed with its filament at a distance of approximately $25 \mathrm{~cm}$ from the lens. The position of the light bulb and lens was optimized around these values for optimal light intensity and -homogeneity on the wafer. To prevent heating of the outside of the etch bath, it was cooled by a flow of air from a ventilator. In some experiments the wafers were illuminated by a $1000 \mathrm{~W}, 230 \mathrm{~V}$ industrial halogen lamp, in front of which a $100 \times 100 \mathrm{~mm}^{2}$ Melles-Griot KG1 tempered heat absorbing filter was mounted. The distances between filament, lens and 


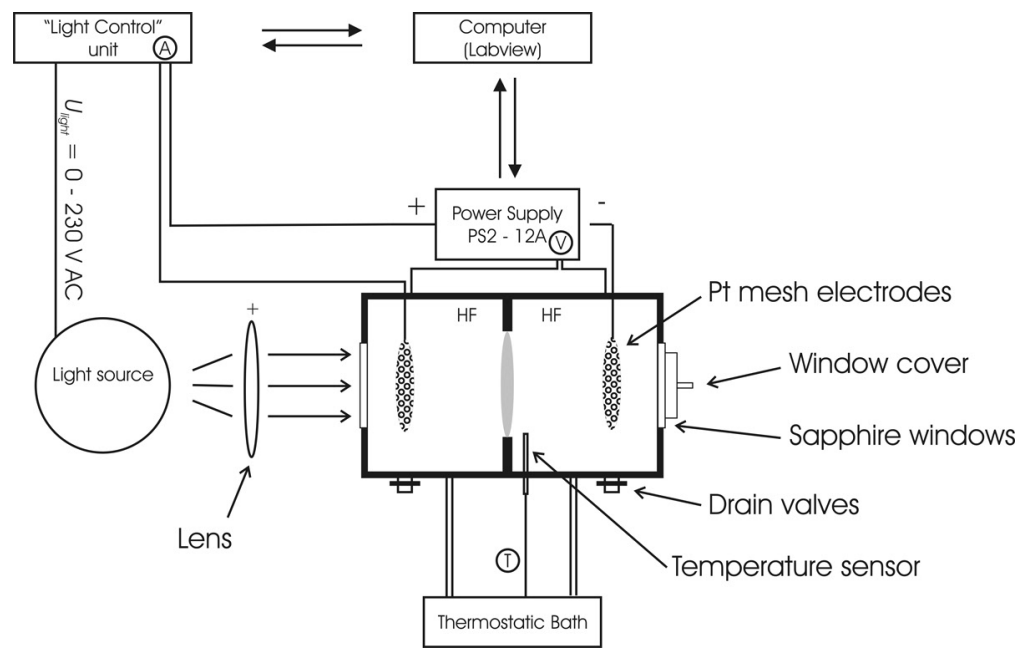

Figure 3.4: Schematic overview of the photo electrochemical etching setup. The black rectangle in the bottom right shows the etch bath, which consists of two separate chambers holding the HF electrolyte. A thermostatic bath regulates the temperature of the electrolyte, measured using an external temperature sensor. The wafer (grey ellipsoid) separates the two chambers using a wafer holder (not shown). Left and right of the sample wafer, the two platinum electrodes are connected to the power supply. These electrodes are sieve-shaped and allow light to pass. The wafer is illuminated through a sapphire window on the left side of the etch bath. The window on the right side of the bath is covered to prevent light from entering. The light intensity incident from the light source is optimized with a lens. The etch bath is connected to a power supply, which is controlled by a personal computer. A Labview program on this computer runs and monitors all processes, except for the temperature regulation. The actual etching occurs in the right etch chamber, in which the electrolyte is stirred using a magnetic stirrer.

etch bath was similar as above, and optimized for optimal light intensity and -homogeneity on the wafer. The light intensity on the wafer was regulated by a home-built Light Control unit, which has two functions:

a) Measuring and converting the current $I$ through the etch bath to a 15-bit signal, which is sent to the Labview program in control of the process. Different possible maximum currents can be selected via a switch board $(10,20,50$, and $100 \mathrm{~mA}$, as well as $0.5,1,6$, and $12 \mathrm{~A})$. The resolution is $0.01 \%$ of the full scale and the accuracy is $0.02 \%$ of the full scale.

b) Adjusting the light intensity $L$ by applying power from 0-230 V AC with a 12-bit resolution. The amount of power to the light source is determined 
by controlling the phase angle of the AC supply.

Two sieve-shaped platinum electrodes with a diameter of $98 \mathrm{~mm}$, placed at a distance of around $8 \mathrm{~cm}$ from the wafer, generate the electric field over the wafer. These electrodes allow light to pass. Power is supplied by a AMMT GmbH PS ${ }^{2}$ power supply, which:

a) Sets the potential $U$ from $0-12 \mathrm{~V}$ with a resolution of $10 \mathrm{mV}$. The accuracy of the indicated value is $0.02 \%+26 \mathrm{mV}$. The current $I$ can be up to $12 \mathrm{~A}$.

b) Measures potential $U$ using remote sensing and returns the measured value to the personal computer.

A personal computer runs a program written at our institute in Labview $7.1^{2}$. The program monitors and controls several processes:

a) It controls both the Light Control Unit and the $\mathrm{PS}^{2}$ power supply.

b) It reads out $U$, and, from the data generated by the Light Control unit, returns a calibrated value for the measured current $I$. The error on the scale bar of $I$ is determined by the calibration in the Labview program and is accurate within at least $2 \%$.

c) It measures $I-U$ curves and saves the data to disk.

d) It runs etching processes, i.e., it sets $U$ and a setpoint current $I_{\text {set }}$ and adjusts the light intensity on the wafer $L$ via the Light Controller using a proportional integral derivative (PID) routine. To do so the program continuously compares $I_{\text {set }}$ and the measured current $I$. When $I<I_{\text {set }}$ the program increases $L$ and vice-versa. During $I-U$ curve measurements the light intensity was set manually on a scale from 0 to $100 \%$.

e) The program stores etching data, consisting of time, $I, I_{\text {set }}, U$, and $L$, and saves the data to disk.

For analysis of the results we convert measured currents $I$ to current densities $J\left(\mathrm{~mA} / \mathrm{cm}^{2}\right)$ by dividing $I(\mathrm{~mA})$ with the exposed surface area $A\left(\mathrm{~cm}^{2}\right)$ of the processed wafer, see equation 3.2 .

To monitor the electrolyte temperature during etching, the etch bath was equipped with a teflon coated temperature sensor. The sensor was connected

\footnotetext{
${ }^{2}$ The Labview program was written by R. Nauta of the "Techno Centrum voor Onderwijs en Onderzoek" (TCO) of the faculties Science and Technology and Electrical Engineering, Mathematics and Computer Science.
} 
Chapter 3. Photo electrochemical etching of pores in silicon

Table 3.1: Overview of wafer types used in the experiments. Listed are the average measured resistivities of the wafers, the average thicknesses, the number of measurements performed on each wafer, and the number of wafers studied.

\begin{tabular}{|l|c|c|}
\hline & Type 1 & Type 2 \\
\hline Resistivity $(\Omega \mathrm{cm})$ & $0.76 \pm 0.022$ & $0.93 \pm 0.017$ \\
\hline Thickness $(\mu \mathrm{m})$ & $698 \pm 20$ & $381 \pm 10$ \\
\hline \# of measurements & 1 & 5 \\
\hline \# of wafers & 2 & 4 \\
\hline
\end{tabular}

to a Phoenix thermostatic bath (P1-C25P) which was used to control the temperature of both HF chambers. Heat is exchanged via tubes mounted at the bottom of both chambers. The temperature of the electrolyte and of the water in the exchange tubes can be varied from 10 to $30^{\circ} \mathrm{C}$. Using a magnetic stirrer the electrolyte was stirred in the right chamber in Figure 3.4 , where the actual etching takes place.

\subsection{Experimental methods}

For this work single crystal silicon wafers were used with a range of thicknesses and resistivities (n-type, single side polished, $<100>$ ). Choosing the correct wafer resistivity is essential, as was discussed in section 3.1.2. We will further address wafer resistivities in our analysis of the etching results in section 3.6. An overview of the wafer types is given in Table 3.1. The diameter of all wafers was $100 \mathrm{~mm}$. A $200 \mathrm{~nm}$ thick silicon nitride $\left(\mathrm{Si}_{3} \mathrm{~N}_{4}\right)$ layer was deposited on the wafers. This material was chosen because of its high stability in a KOH etching bath [148]. Subsequently, photoresist (PEK 445) and topcoat (Aquatar) were spincoated on the wafers. The resist was patterned using an ASML PAS5500/700 deep UV step and scan system ${ }^{3}$.

After patterning, the silicon nitride layer was etched in a Elektrotech Twin system PF 340, using a reactive ion etching process with the configuration and parameters listed in Table 3.2. After the reactive ion etch, the remaining photoresist was exposed to oxygen plasma from a Tepla 300E dry etcher until all remaining photoresist was removed. The applied settings are listed in Table 3.3.

To remove a thin layer of silicon nitride still present on the bottom of the holes in the mask, the wafers were dipped in an aqueous $50 \% \mathrm{HF}$ solution

\footnotetext{
${ }^{3}$ Patterning was performed by R. Voets and I. D. Setija of ASML B.V.
} 
Table 3.2: Reactive ion etching settings with which the silicon nitride layer was etched after deep UV lithography. A general description of reactive ion etching is available in Chapter 4

\begin{tabular}{|c|c|}
\hline Parameter & Setting \\
\hline Chamber & "clean chamber" \\
\hline Electrode & quartz \\
\hline Substrate temperature & $10^{\circ} \mathrm{C}$ \\
\hline Pressure & 10 mTorr \\
\hline Etchant flow & $5 \mathrm{sccm} \mathrm{O}_{2}$ and 25 sccm $\mathrm{CHF}_{3}$ \\
\hline Plasma power & $75 \mathrm{~W}(13.56 \mathrm{MHz}$ radio frequency $)$ \\
\hline Duration & $2 \frac{1}{2}$ minutes \\
\hline
\end{tabular}

Table 3.3: Tepla 300E dry etcher settings with which the remaining photoresist was removed after reactive ion etching of the silicon nitride layer.

\begin{tabular}{|c|c|}
\hline Parameter & Setting \\
\hline Plasma power & around $300 \mathrm{~W}$ \\
\hline Oxygen flow & $50 \%$ (mass flow controller) \\
\hline Pressure & typically 1.25 mbar \\
\hline
\end{tabular}

for $2 \frac{1}{2}$ min. After preparation of the silicon nitride mask, the pore tips were etched by immersing the wafers for $2 \frac{1}{2}$ minutes in a $25 \%$ (wt) $\mathrm{KOH}$ solution at $75^{\circ} \mathrm{C}$. Subsequently the wafers were cleaned for 15 minutes with a 5:1:1 solution of $\mathrm{H}_{2} \mathrm{O}, \mathrm{HCl}(32 \%)$, and $\mathrm{H}_{2} \mathrm{O}_{2}(31 \%)$ at a temperature exceeding $80^{\circ} \mathrm{C}$. After all separate chemical preparative steps the wafers were rinsed with demineralized water.

The prepared wafers were used in two types of experiments, which are

Table 3.4: Contents of the aqueous 5\% HF solution (2.5M) that was used for etching. HF 50\%(wt) (Merck VLSI Selectipur), ethanol (H. Assink B.V., technical grade), and demineralized water were used as available in the MESA+ cleanroom. Triton X-100 was purchased from Aldrich (product number 23,472-9).

\begin{tabular}{|c|c|c|c|c|c|}
\hline & HF $50 \%(\mathrm{wt})$ & Ethanol & $\mathrm{H}_{2} \mathrm{O}$ & Triton X-100 & Total \\
\hline Added volume & $500 \mathrm{ml}$ & $250 \mathrm{ml}$ & $4250 \mathrm{ml}$ & 20 drops & $5 \mathrm{l}$ \\
\hline
\end{tabular}


described here in general. Further details will be given in the text where appropriate. All experiments were done in an aqueous 5\% HF solution, prepared as outlined in Table 3.4.

- In $I-U$ curve measurements, the potential over the platinum electrodes is scanned upwards from $0 \mathrm{~V}$ to a chosen potential and back, with scanrates of typically $50 \mathrm{mV} / 1000 \mathrm{~ms}$. Simultaneously the current $I$ is measured. In most cases the scan was repeated for a number of times. The backside of the wafer was either continuously illuminated with $100 \%$ light intensity, or kept in the dark. The $I-U$ scans are plotted as current density $J$ versus potential $U$, according to equation 3.2. $I-U$ measurements were also performed without a wafer, but with wafer holders inserted, and in the dark. The value of the potential to which was scanned depended on the experiment.

- During actual etching of pores a constant potential $U$ was set over the platinum electrodes and a set point current $I_{\text {set }}$. By controlling the intensity of the backside illumination $L$, the measured current $I$ was maintained as close as possible to $I_{\text {set }}$. The wafers were etched for etch durations of up to 1 hour.

To describe the experiments and the subsequent analysis twelve measured and calculated electronic parameters are differentiated, see Table 3.5. This differentiation will be used from this point on.

After etching, the samples were analyzed using a LEO 1550 high-resolution scanning electron microscope, optical microscopy using a ME600L Nikon Eclipse optical microscope, or both. The optical microscope is equipped with CFI LU Plan BD 5x - 100x objectives, and a DXM-1200F digital camera. Where cross-sections of pores were imaged, the wafers were cleaved prior to analysis.

\subsection{Fabrication of pore tips}

During photo electrochemical etching, the position of the etched pores is determined by the pattern of the pore tips on the sample wafers. Our pattern was a centered rectangular lattice with a $2 \mathrm{~mm}$ lattice symmetry [117]. The angle $\varphi$ between the two axes of the primitive cell is $84.5^{\circ}$. This pattern was chosen as it always cleaves through a set of pores for convenient structural analysis, while it only slightly differs from a square pattern. Figure 3.5(A) displays the pattern in the photoresist after deep UV step and scan processing. On a wafer, many different periodic arrays of holes with a 


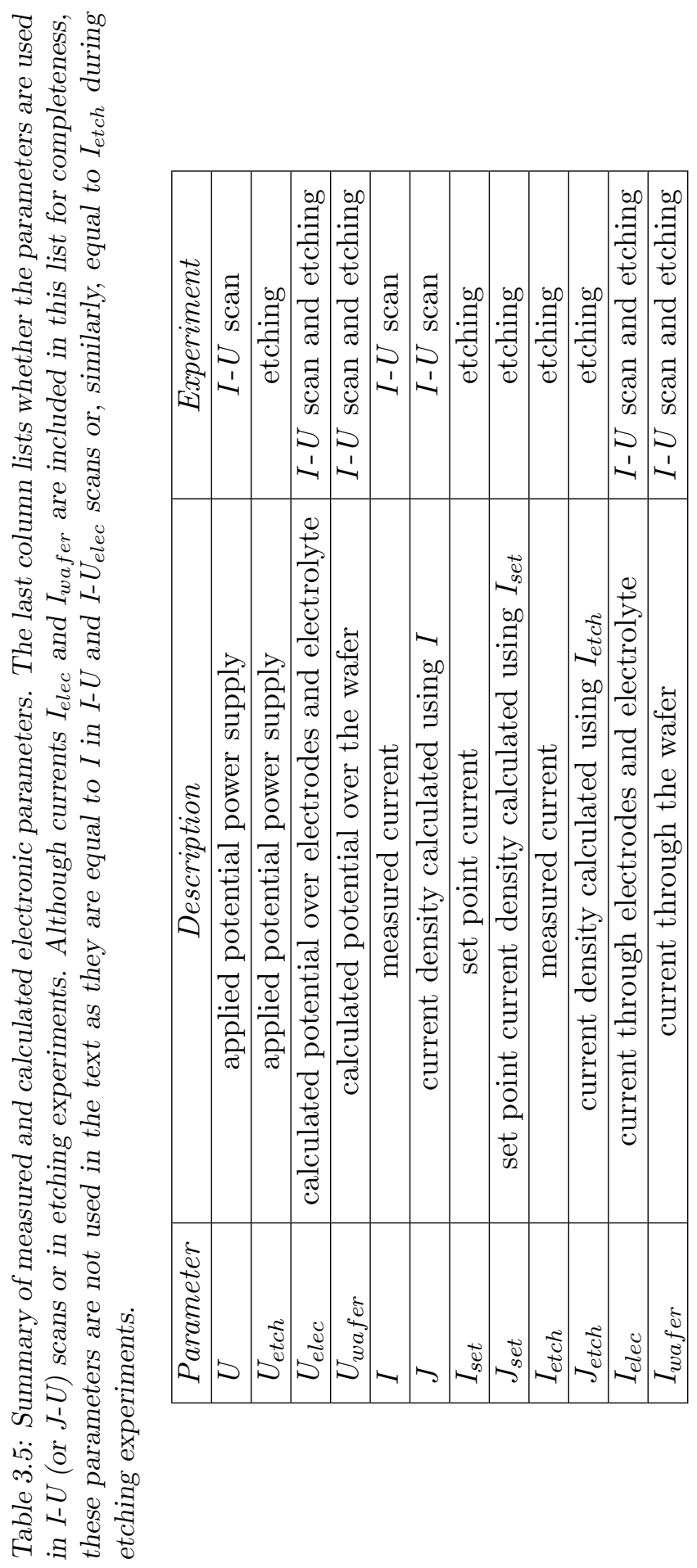



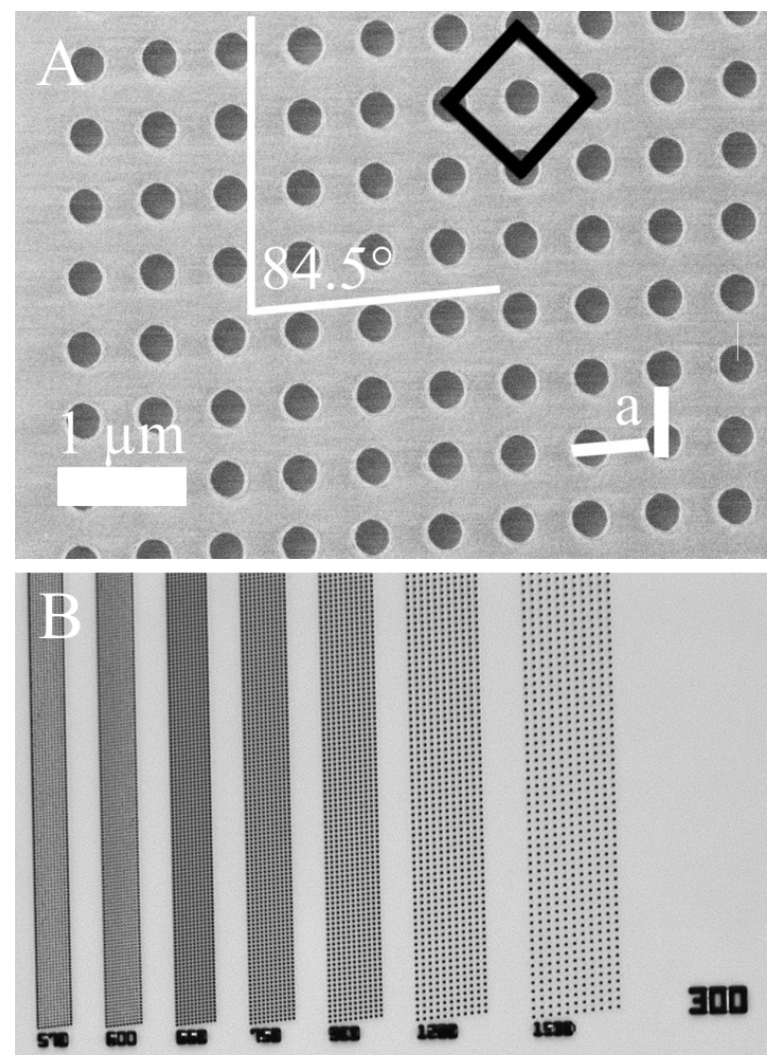

Figure 3.5: A) Scanning electron micrograph of the patterned photoresist after step and scan processing. The holes in the photoresist are oriented in a centered rectangular lattice, outlined in black. Outlined in white are the two axes of the primitive cell, with angle $\varphi=84.5^{\circ}$. The pitch is the shortest distance between two holes and is indicated by the two short white bars indicated a. Here the pitch equals $a=550 \pm 11 \mathrm{~nm}$ and the diameter is $D=280 \pm 14.5 \mathrm{~nm}$. B) The periodic patterns of holes are arranged in different sets of diameter-pitch combinations. In each set the separate lines have the same specified diameter and varying pitches. Here the specified diameter is $D_{\text {spec }}=300 \mathrm{~nm}$, with pitches varying from $a=570$ $\mathrm{nm}$ to $1500 \mathrm{~nm}$. 
Table 3.6: Overview of relevant specified diameter-pitch combinations on the wafer. Arrays with a ratio of specified diameter $D_{\text {spec }}$ to pitch a of 1.9 can not be used due to trench formation during the $\mathrm{KOH}$ etch step. Structures with too high pitches are of limited interest to us, because they have photonic properties at long wavelengths outside the telecommunication regime. Outlined in gray are the diameter-pitch combinations that we are investigating.

\begin{tabular}{|c|c|c|c|c|c|c|c|}
\hline $\mathrm{D}_{\text {spec }}(\mathrm{nm})$ & \multicolumn{7}{|c|}{ Pitches $a(\mathrm{~nm})$} \\
\hline 500 & 2500 & 2000 & 1500 & 1250 & 1100 & 1000 & 950 \\
\hline 350 & 1750 & 1400 & 1050 & 875 & 770 & 700 & 665 \\
\hline 300 & 1500 & 1200 & 900 & 750 & 660 & 600 & 570 \\
\hline 250 & 1250 & 1000 & 750 & 625 & 550 & 500 & 475 \\
\hline 220 & 1100 & 880 & 660 & 550 & 484 & 440 & 418 \\
\hline 200 & 1000 & 800 & 600 & 500 & 440 & 400 & 380 \\
\hline \hline$\left(D_{\text {spec }} / a\right)$ & 5 & 4 & 3 & 2.5 & 2.2 & 2 & 1.9 \\
\hline
\end{tabular}

wide range of pitches $a$ and a range of hole diameters $D_{\text {spec }}$ are specified. The pitch $a$, also referred to as interpore distance, is the shortest distance between two holes, and is indicated in Figure 3.5(A). Figure 3.5(B) is an optical microscopy image showing arrays of holes with one particular specified diameter and varying pitches. The length of the arrays of holes extend over more than $15 \mathrm{~mm}$, that is more than 15000 pores. Such large structures are made without stitching, which is an advantage of deep UV lithography over e-beam lithography. Moreover, the simultaneous lithography of different diameter-pitch combinations is an advantage over laser interference lithography.

Table 3.6 shows the relevant specified diameter-pitch combinations available on the wafer. Only arrays with a ratio $\left(D_{\text {spec }} / a\right) \leq 1.9$ can not be used due to trench formation during the $\mathrm{KOH}$ etch step. Structures with too high pitches are of limited interest to us, because they will have photonic properties at long wavelengths, outside the telecommunication regime. Outlined in gray are the pitches that are suitable for our purposes.

With the pattern in the photoresist as a blueprint, the pore tips were fabricated. Figure 3.6 shows scanning electron micrographs of various steps in the pore tip formation process. In Figure 3.6(A) the holes in the silicon nitride are shown after they where etched. Visible in the picture are the silicon wafer (bottom), the nitride layer with holes (middle), and the remaining photoresist (top). The Figure shows that the sidewalls of the holes in the silicon nitride are straight. Initial experiments indicated that a thin layer of 


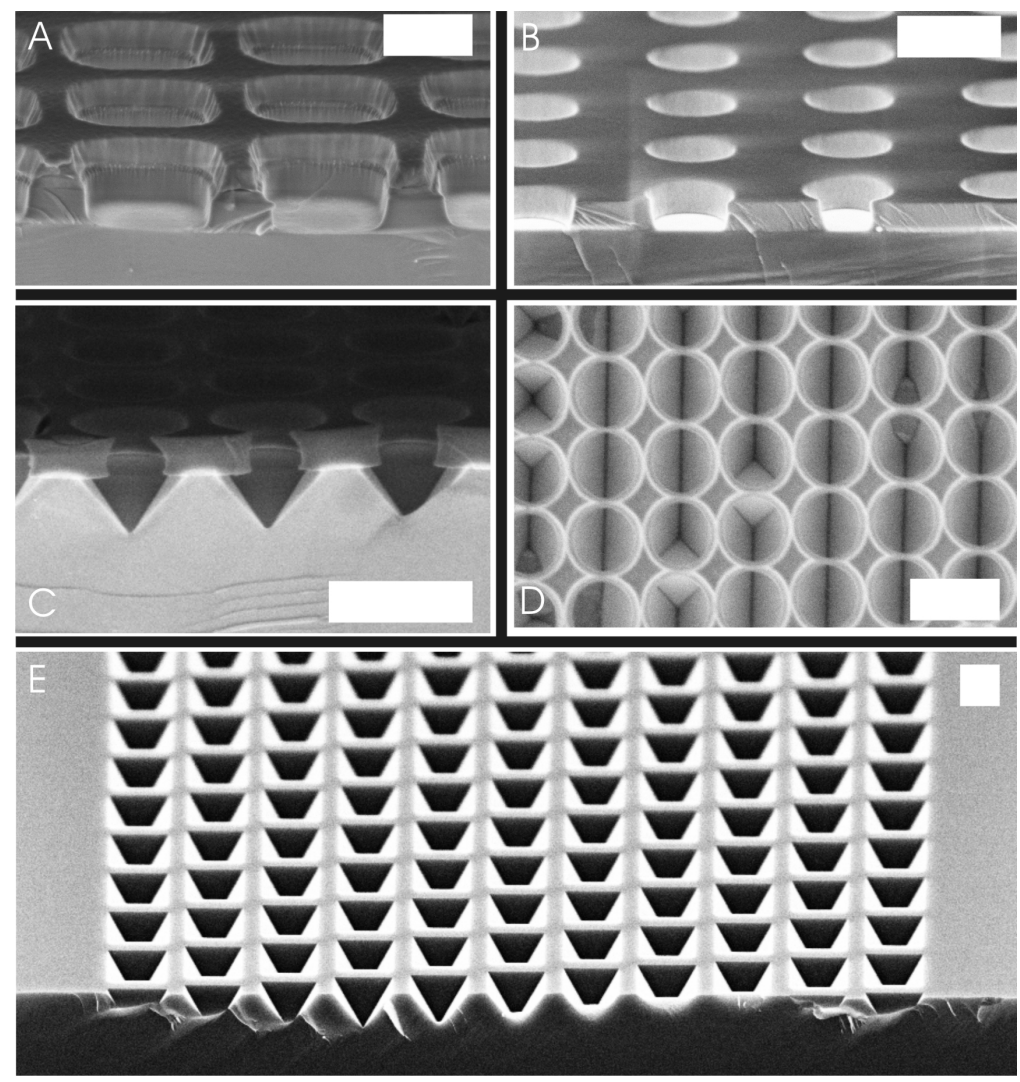

Figure 3.6: Scanning electron micrographs of various steps in the fabrication process of the inverted pyramid shaped pore tips. All scale bars equal $500 \mathrm{~nm}$. A) The holes in the silicon nitride after they where etched. Visible are the silicon wafer (bottom), the nitride layer with holes (middle), and the remaining photoresist (top, vertically striped edges). The pitch $a=1000 \pm 20 \mathrm{~nm}$. B) Holes in the silicon nitride mask after removal of the photoresist. C) Pore tips resembling inverted pyramids etched with $\mathrm{KOH}$. The silicon nitride mask is unaffected and can still be seen. The pitch a $=484 \pm 9 \mathrm{~nm}$. D) At $\left(D_{\text {spec }} / a\right)<2$, trenches instead of pore tips start to appear, as shown in this topview. Trenches are not usable for the photo electrochemical etching process. The circles visible in the picture are the boundaries of the holes in the silicon nitride mask, which was not yet removed. It can already be seen that the boundaries touch and nearly overlap. Here $D_{\text {spec }}=200 \mathrm{~nm}$ and the specified $a=$ $380 \mathrm{~nm}$. E) Highly defined pore tips in silicon with $a=950 \pm 19 \mathrm{~nm}$ after removal of the silicon nitride layer. 
silicon nitride was still present on the bottom of these holes, which prevented further pore tip formation with $\mathrm{KOH}$. Therefore, we decided to remove the remaining photoresist and subsequently etch this small layer of silicon nitride with an aqueous $50 \% \mathrm{HF}$ solution. In Figure 3.6(B) the result of this etching step is shown. The photoresist has been removed completely, and the holes in the silicon nitride are still well defined, although the diameter of the holes has increased due to the isotropy of the HF etching. After opening of the holes in the silicon nitride, the pore tips were formed by exposure of wafer to a $\mathrm{KOH}$ solution at elevated temperature. This solution etches silicon $6 \cdot 10^{2}$ times faster in the $\langle 110\rangle$ direction than in the $<111>$ direction [123], which results in the inverted pyramid-like pore tips shown in Figure 3.6(C). The angle of the sides of the inverted pyramides with the wafer normal is around $35^{\circ}$. The selectivity of silicon etching compared to the etching of silicon nitride is high, which is confirmed by the observation that the thickness of the silicon nitride layer on top of the inverted pyramids is almost unmodified. The surface of the base of the inverted pyramids is defined by the diameter of the holes in the silicon nitride mask. The higher the diameter of the hole, the larger the base of the inverted pyramid. Consequently, the height from the base to the apex is determined by the diameter of the holes in the mask. We find typical heights of a few hundreds of nanometers. The position of these pore tips, determined by the interpore distance $a$, defines where etching occurs when the correct experimental parameters are chosen.

Figure 3.6(D) shows what happens if the bases of the inverted pyramids overlap. Overlapping is either due to the diameters of the mask being increased too much, or occurs during etching with $\mathrm{KOH}$ due to the small amount of lateral etching. The Figure shows that under these circumstances trenches start to appear. Obviously these trenches are not usable as starting points for the photo electrochemical etching of pores. An analysis of the diameter-pitch combinations available on the wafer reveals that the problem of trench formation is avoided when the ratio of specified diameter $D_{\text {spec }}$ to pitch is larger than 2 (see Table 3.6).

After removal of the remaining silicon nitride layer, the arrays of pore tips are ready for use, see Figure 3.6(E). The patterning procedure results in well defined inverted pyramid shaped pore tips, with a wide range of pitches, which allows for an extensive analysis of the etching process as a function of interpore tip distance. 


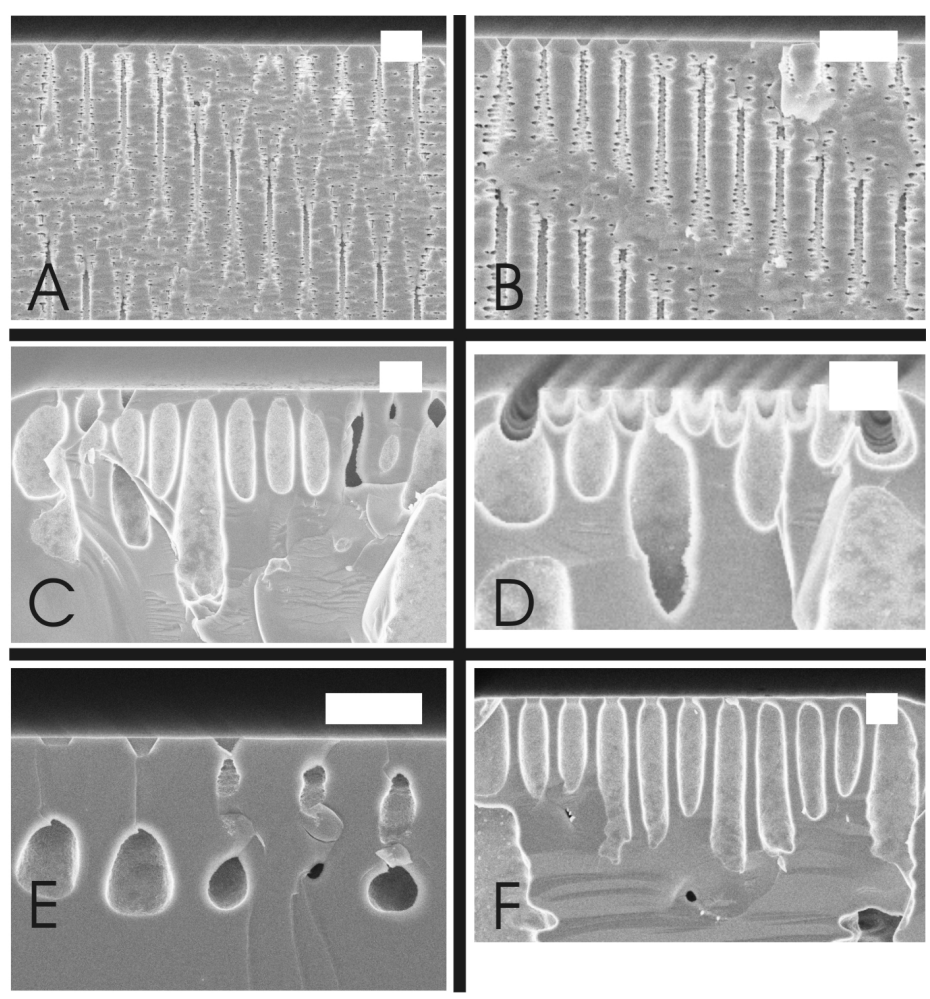

Figure 3.7: Scanning electron micrographs of a patterned wafer of type 1 which was etched on several positions with different potentials $U_{\text {etch }}$ and set current densities $J_{\text {set }}$. The exposed area was $A=3.8 \mathrm{~cm}^{2}$ and the total etch time was $3600 \mathrm{~s}$. The scale bars equal $2 \mu \mathrm{m}$. Detailed experimental and pore tip conditions of each facet have been summarized in Table 3.7. Comparing A) with $C$ ) and B) with D) shows the effect of reducing $U_{\text {etch }}$ with unchanged $J_{\text {set }}$ at different interpore distances. $E$ ) shows what happens when both $U_{\text {etch }}$ and $J_{\text {set }}$ are reduced compared to $A$ ) and $C$ ). In $F$ ) the best pores we obtained are shown.

\subsection{Etched pores}

This section contains what we consider to be our best etching results to date. Figure 3.7 shows scanning electron micrographs of pores etched in a wafer of type 1 with different potentials $U_{\text {etch }}$ and different current densities $J_{\text {etch }}$, determined by the set current density $J_{\text {set }}$. In all cases pore growth occurred, confirming that the set current density $J_{\text {set }}$ is below the critical current density $J_{p s}$. For clarity, the experimental parameters corresponding to the different facets in Figure 3.7 are summarized in Table 3.7. 


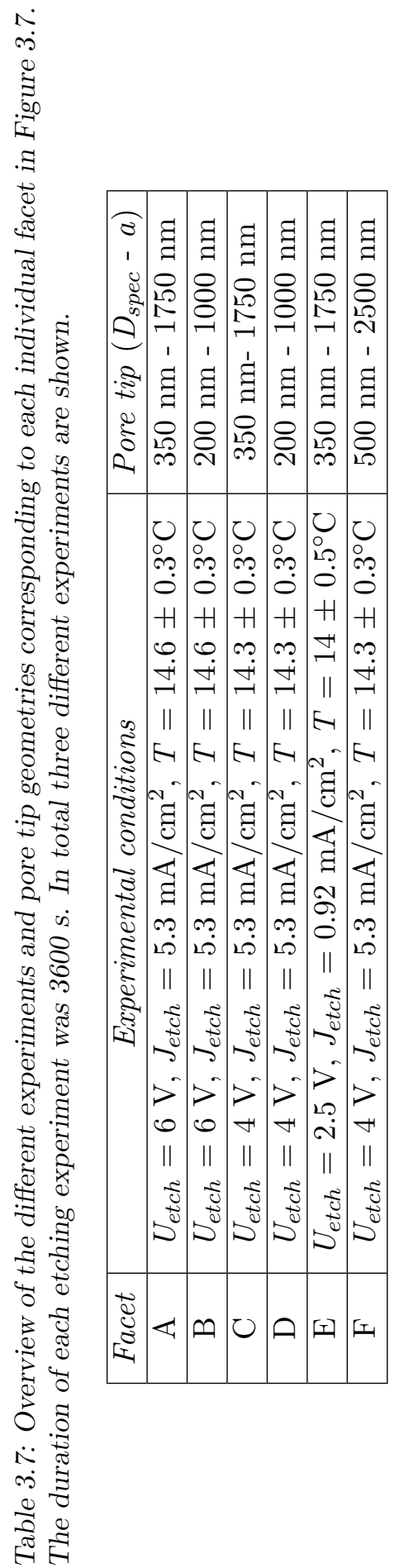




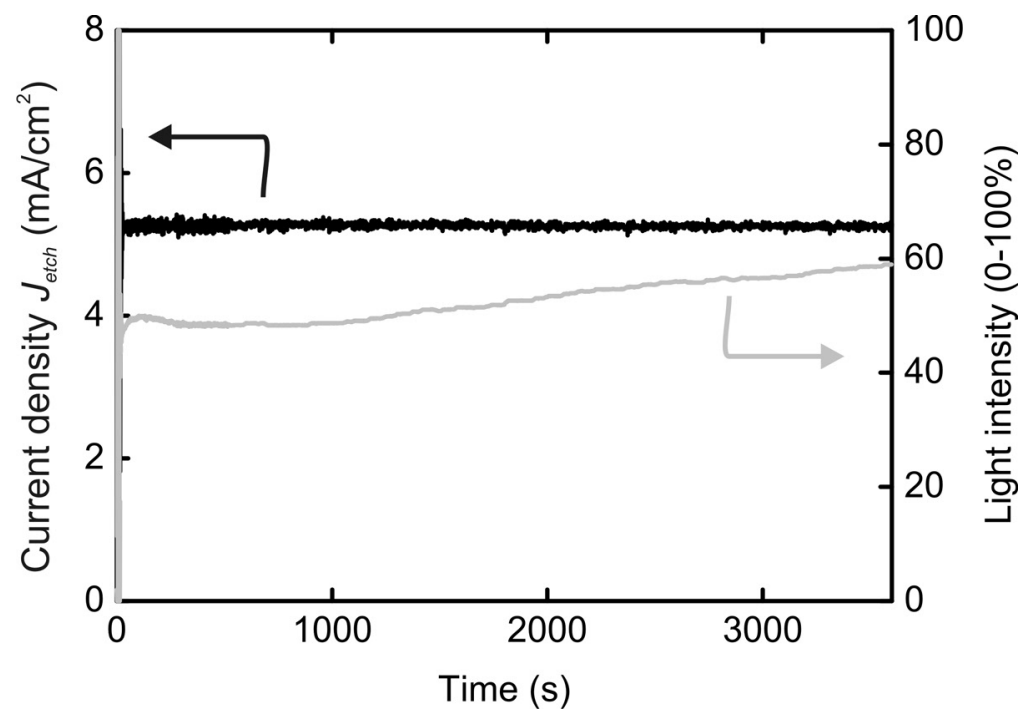

Figure 3.8: Measured current density $J_{\text {etch }}$ versus etching time during etching at a fixed potential of $U_{\text {etch }}=4 \mathrm{~V}$ (black curve). Here, a patterned wafer of type 1 was etched for $3600 \mathrm{~s}$. The set point current $J_{\text {set }}$ was $5.3 \mathrm{~mA} / \mathrm{cm}^{2}$. The exposed area was $A=3.8 \mathrm{~cm}^{2}$. The temperature of the electrolyte was $14.3 \pm 0.3^{\circ} \mathrm{C}$. The grey curve shows the light intensity $L$ on the backside of the wafer versus etching time on a scale of $0-100 \%$ of maximum power to the light bulb.

The pores shown in Figures 3.7(A) and (B) are from the same experiment, but have different interpore distances. These were etched with a relatively high potential $U_{\text {etch }}=6 \mathrm{~V}$, resulting in an etching rate of around $0.7 \mu \mathrm{m}$ per minute. Although these pores are deep, they are highly branched.

By etching with a lower potential $U_{\text {etch }}=4 \mathrm{~V}$ at equal current density, branching is eliminated, see Figure $3.7(\mathrm{C})$. However, lowering the potential resulted in loss of selective growth of pores at pore tips with lower interpore distances, see for example Figure 3.7(D) where an (intended) interpore distance of $a=1000 \mathrm{~nm}$ is shown. This Figure shows that pore growth is not initiated at each pore tip, but only at a few. In addition, the etching rate is significantly reduced. In Figure $3.7(\mathrm{~F})$ an example of nicely grown pores is shown etched at the same experimental conditions as (C) and (D). These pores start to grow at the pore tips and continue to grow without joining or branching. In this case the interpore distance was $a=2.5 \mu \mathrm{m}$. The diameter of the middle 9 pores was estimated to be $D_{\text {pore }}=1.7 \pm 0.17 \mu \mathrm{m}$.

Figure 3.7(E) shows what happens when both $U_{\text {etch }}$ and $J_{\text {set }}$ are reduced compared to $(\mathrm{A})$ and $(\mathrm{C})$. Although pore growth occurs at the pore tips, the 


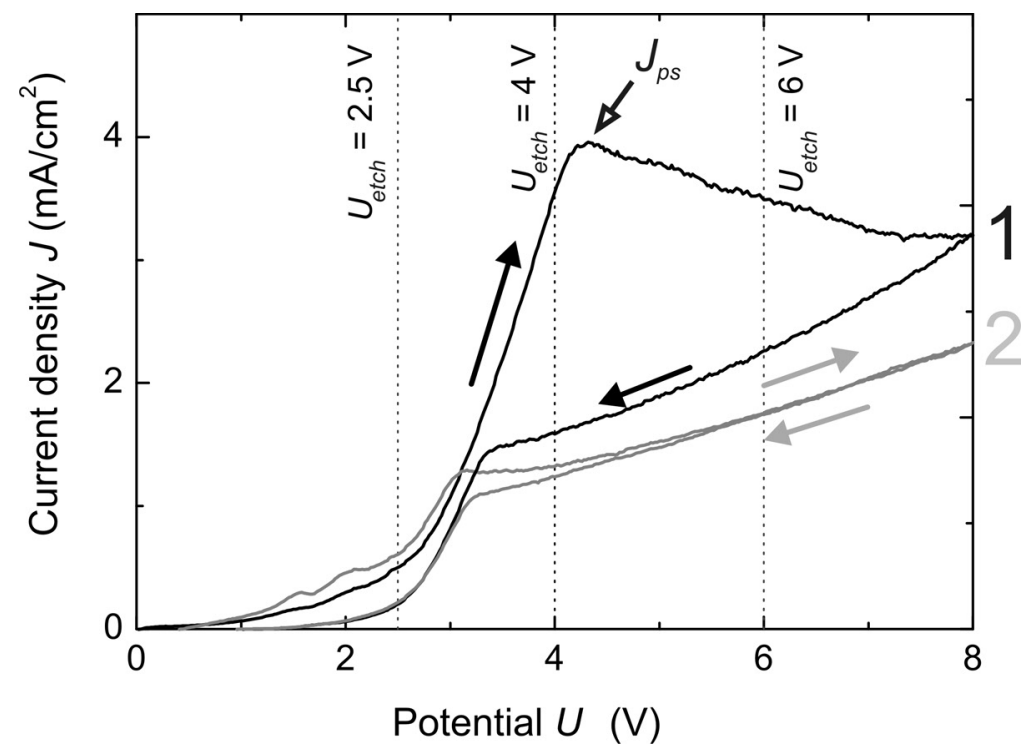

Figure 3.9: $J$ versus $U$ curve of a wafer which was used for the experiments shown in 3.7. The black lines are the first upward-and downwards scans and the grey lines are the second scans. The curves were measured at temperature $T=15.5 \pm 0.5^{\circ} \mathrm{C}$. The dotted vertical lines show the potentials $U_{\text {etch }}$ for the three etching experiments described in this section. The values of $U_{\text {etch }}$ were chosen in very distinct regimes of this curve. The top of the peak is tentatively identified as the critical current density $J_{p s}$, indicated by the open arrow.

etching rate is even further reduced. In addition, the pores are no longer circular in shape, but get a droplet-like geometry. Judging from the three experiments reported in this section, the experimental conditions with which the pores shown in $(\mathrm{C}),(\mathrm{D})$, and $(\mathrm{F})$ were etched are the most favorable.

A typical time trace of an etching experiment is shown in Figure 3.8. In this Figure the development of the current density $J_{\text {etch }}$ and the light intensity $L$ are shown for the full duration of the etching experiment. Here, the current density was successfully kept at its set value of $J_{\text {set }}=5.3 \mathrm{~mA} / \mathrm{cm}^{2}$ by continuously monitoring and adjusting the light intensity $L$. The potential $U_{\text {etch }}$ was kept constant at $4 \mathrm{~V}$.

\subsection{Discussion}

To attempt a detailed analysis of the etching results we compare the results at different etch potentials $U_{\text {etch }}$ with a $J$ - $U$ curve of the etched wafers, see 


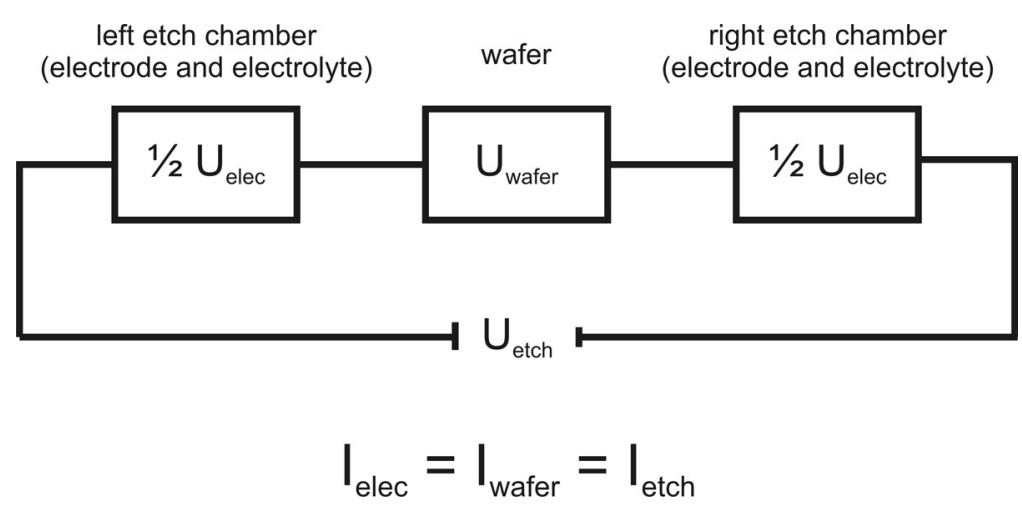

Figure 3.10: The separate components of the etching system (electrode and electrolyte, and the wafer) are in series. In this situation equations 3.10 and 3.11 are valid.

Figure 3.9. The etch potentials $U_{\text {etch }}$ are chosen around the dominant peak in the measured $J$ - $U$ curve, which is tentatively identified as the critical current density $J_{p s}$. Different results were expected based on the chosen potentials $U_{\text {etch }}$, which are in very distinct regimes on this curve. However, in hindsight we realized the importance of accurately correcting the set etch potential $U_{\text {etch }}$ for the potential over the electrolyte and electrodes $U_{\text {elec }}$, which yields the true potential over the wafer during etching $U_{w a f e r}$.

As illustrated in Figure 3.10 these components of the etching system are in series. In this situation the following equations apply for the potential ${ }^{4}$ :

$$
U_{\text {wafer }}=U_{\text {etch }}-U_{\text {elec }} .
$$

and for the current:

$$
I_{\text {etch }}=I_{\text {wafer }}=I_{\text {elec }} \text {. }
$$

At a set potential $U_{\text {etch }}$, the resulting current is determined by the resistance of the entire system, i.e., the electrolyte, the electrodes, and the wafer. As we will see, this dependence is non-trivial.

The set etch potential $U_{\text {wafer }}$ is the relevant quantity to consider when analyzing the etching results and is compared to a $J$ - $U_{\text {wafer }}$ curve. This analysis is achieved by the following sequence of steps:

i) $I-U_{\text {elec }}$ curves are measured in the situation where no wafer is present in wafer holder.

\footnotetext{
${ }^{4}$ These equations are defined for etching experiments, but are equally suitable for $I$ -

$U$ curve measurements by simply replacing $U_{\text {etch }}$ with $U$ and $I_{\text {etch }}$ with $I$.
} 
ii) $J$ - $U_{\text {wafer }}$ curves are calculated using measured $I$ - $U$ curves (with a wafer present) and the results from i).

iii) The set potential during etching $U_{\text {etch }}$ is corrected for the potential over the electrolyte and electrodes $U_{\text {elec }}$ to yield the etching potential over the wafer $U_{\text {wafer }}$.

iv) The wafer potentials during etching $U_{\text {wafer }}$ are plotted in the calculated $J$ - $U_{\text {wafer }}$ curves and the etching results are analyzed.

These four steps are discussed in the remainder of this section. The performed calculations rely on the conditions described by equations 3.10 and 3.11. In particular, to calculate the potential over the wafer $U_{\text {wafer }}$, the measured potentials can only be subtracted for equal measured currents in the relevant $I$ - $U$ scans. Note that in these calculations we have to use currents, instead of the preferred current densities.

\section{Step i: measure $\mathbf{I}-\mathbf{U}_{\text {elec }}$ curves}

In order to determine the relation between the potential over the electrolyte and electrodes $U_{\text {elec }}$ and the current $I, I$ - $U_{\text {elec }}$ curves were measured in the situation where no wafer is present in the wafer holder. Unfortunately, the measured $I$ versus $U_{\text {elec }}$ curves are not only very intricate, but also show hysteresis and a sweep-dependent component, see Figure 3.11. The Figure shows two sweeps, consisting of both upward- and downwards scans, for experiments where (A) the full wafer holder was equipped and (B) the test wafer holder was equipped. In the later case the measured current is lower as expected, but not proportional to the reduction of the surface area of the opening in the wafer holder. By equipping the test- instead of the full wafer holder, the open area is reduced more than 15 times, whereas the maximum current is reduced by around 3 times, which is at present not understood. Significant hysteresis is visible between the upward- and downward scans. Such hysteresis is typically the result of insulating layers on the electrode surfaces, but the exact cause remains unidentified. Furthermore Figure 3.11 shows that although in both experiments the downward scans are equal, the first upward scan is lower than the second for potentials up to $2 \mathrm{~V}$. Our experiments show that the $3^{r d}$ sweep is typically equal to the $2^{\text {nd }}$. This means that to correctly obtain the potentials over the wafer $U_{w a f e r}$ at equal measured currents, we have to calculate each separate upward and downward scan, i.e., the first upward scan of the electrolyte is used to correct for the potential in the first upward scan of the electrolyte-wafer system and so on. 


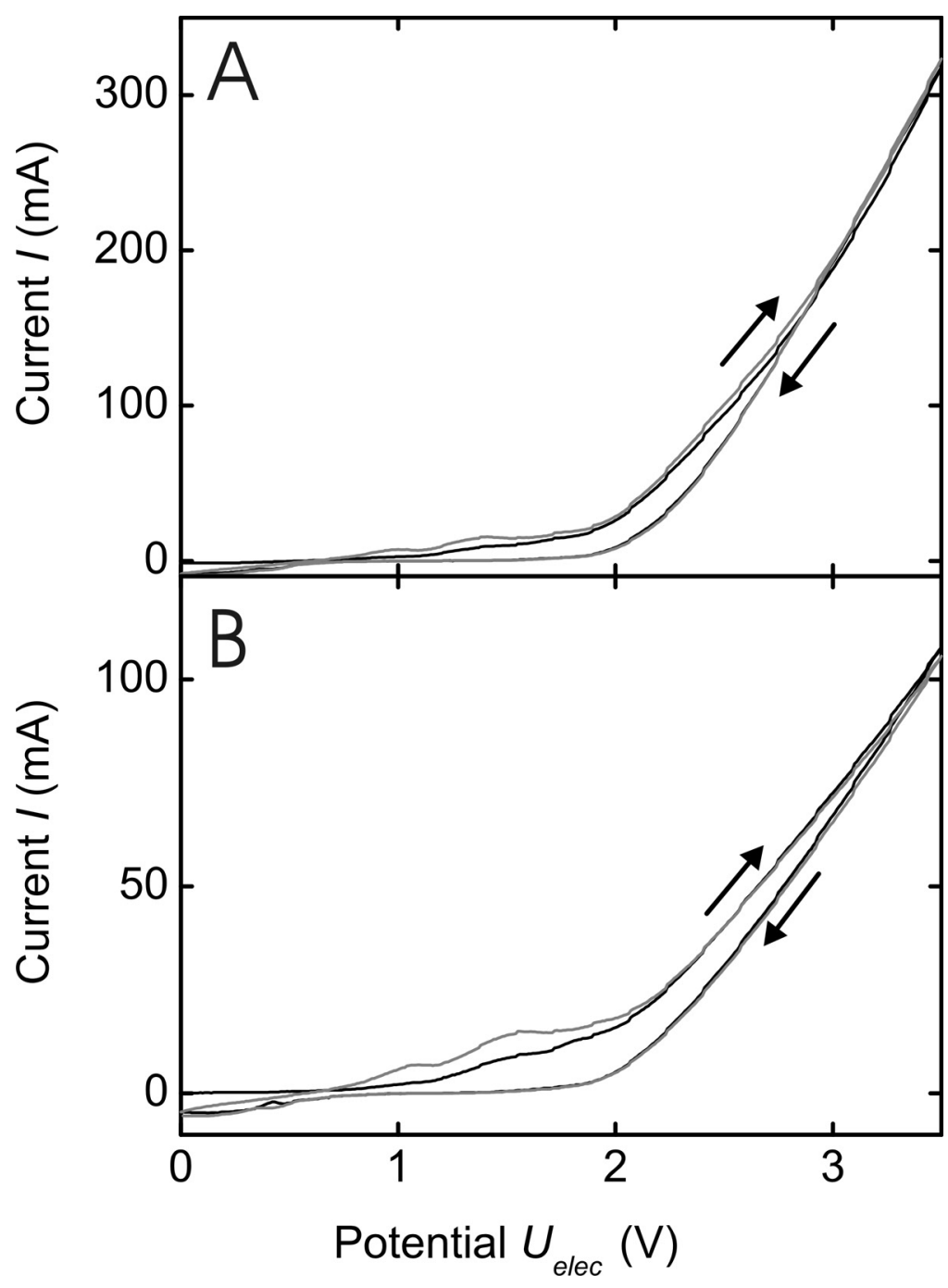

Figure 3.11: I versus $U_{\text {elec }}$ curves of the electrolyte. The black lines are the first upward- and downwards scans and the grey lines are the second scans. A) Scan performed with the full wafer holder. B) Scan performed with the test wafer holder with a smaller exposed surface area. In this case the measured current I is significantly lower. The arrows indicate the direction of the upward-and downward scans. These curves were measured at temperatures $T=14.1 \pm 0.1^{\circ} \mathrm{C}$. 


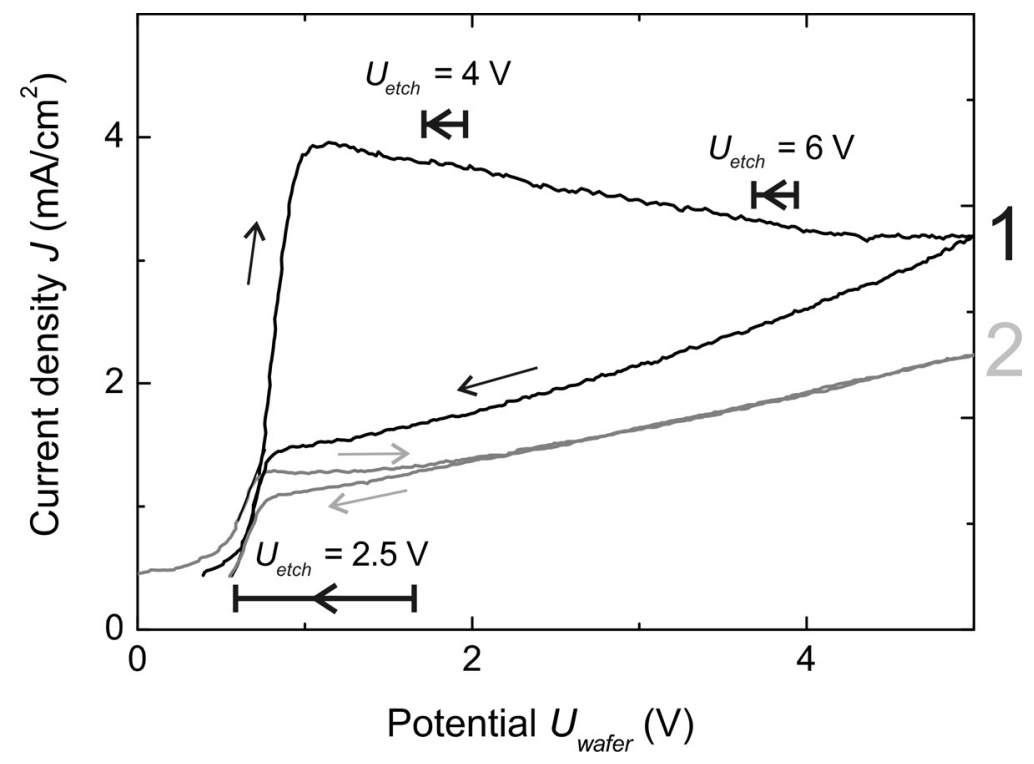

Figure 3.12: $J$ versus $U_{\text {wafer }}$ curve of a wafer which was used for the experiments shown in 3.7. The black lines are the first upward- and downwards scans and the grey lines are the second scans. These curves were measured at temperature $T=15.5 \pm 0.5^{\circ} \mathrm{C}$. The horizontal bars indicate the potential ranges over the wafer $U_{\text {wafer }}$ during the actual etching experiments. The wafer potential during etching $U_{\text {wafer }}$ was determined by correcting the etch potential $U_{\text {etch }}$ for the potential over the electrolyte $U_{\text {elec }}$ at equal measured currents. It is expected that during etching $U_{w a f e r}$ decreases as indicated by the arrow, see Step iii. After a rapid initial decrease, this decrease will be more slow and during the longest part of the etching process $U_{\text {wafer }}$ will be in the lower part of the range shown.

\section{Step ii: calculate $\mathbf{I}-\mathbf{U}_{\text {wafer }}$ curves}

To calculate the potential over the wafer $U_{w a f e r}$, equation 3.10 is used, which is valid for equal currents, i.e., $U$ and $U_{\text {elec }}$ can only be subtracted when the current $I$ during both measurements is equal. To this end, the measured $I-U$ and $I-U_{\text {elec }}$ curves are reduced to $U(I)$ functions ${ }^{5}$. These functions are then used to calculate $U$ and $U_{\text {elec }}$ at equal currents. Subsequently, $U_{\text {wafer }}$ can be calculated for each of these currents.

After the calculations, the currents $I$ were converted to current densities $J$ in order to plot the resulting $J$ - $U_{\text {wafer }}$ curves, see Figure 3.12 . The characteristical peaks in Figure 3.9 at $4.3 \mathrm{~V}$ for the $1^{\text {st }}$ upward scan and at 3.3

${ }^{5}$ The measured data was reduced to $4^{\text {th }}$ order polynomial functions. The polynomial constants were determined with 8 digit precision. 
$\mathrm{V}$ for the $2^{\text {nd }}$, appear at almost equal $U_{\text {wafer }}$ in Figure $3.12(0.9 \mathrm{~V}$ and 0.8 $\mathrm{V}$, respectively), which is expected based on the (tentative) assumption that these peaks represent $J_{p s}$.

\section{Step iii: determine $\mathbf{U}_{\text {wafer }}$ of the etching experiments}

The etching experiments are defined by a set potential $U_{\text {etch }}$ and a set current $I_{\text {set }}$. Using the $I$ - $U_{\text {elec }}$ curves of the electrolyte, we can obtain $U_{\text {wafer }}$ of these etching experiments by again correcting for the potential $U_{\text {elec }}$ found at the current equal to the etching current $I_{\text {etch }}$. For this we assume that second and consecutive $I-U_{\text {elec }}$ sweeps of the electrolyte are the most accurate. However, there is no means to decide whether the potential $U_{\text {elec }}$ during etching should be calculated using an upward scan or a downward scan. Therefore, the potential $U_{\text {elec }}$ will be assumed to be in the range defined by both the upward and downward scan, and a range has to be given for $U_{\text {wafer }}$ during the etching experiment. In Figure 3.12 these ranges are depicted by the horizontal bars. For the $U_{\text {etch }}=2.5 \mathrm{~V}$ experiment, the range over which $U_{\text {wafer }}$ is expected to vary is rather broad, from 0.59 to $1.65 \mathrm{~V}$. For the $U_{\text {etch }}=4$ and $6 \mathrm{~V}$ experiments, the range is better defined from 1.7 to $1.96 \mathrm{~V}$, and 3.68 to $3.94 \mathrm{~V}$ respectively.

From experiments where a constant potential $U_{\text {elec }}$ was applied over the electrolyte without the presence of any wafer, we observed that the current through the system systematically decreases with time, see Figure 3.13. However, in an etching experiment the current is kept constant. The observed time-dependent behavior in Figure 3.13 suggests that in this case the potential $U_{\text {elec }}$ increases with time. Consequently, since during an etching experiment the total potential $U_{\text {etch }}$ is kept constant, $U_{\text {wafer }}$ decreases. In Figure 3.12 this means that $U_{\text {wafer }}$ will move from right to left in its defined range and probably $U_{\text {wafer }}$ will be close to the lower value in the range for most of the etch duration.

\section{Step iv: analysis of the etching results}

Now we can proceed by comparing the geometry of pores with the position of etch potentials $U_{w a f e r}$ on the $J$ - $U_{\text {wafer }}$ curve, as shown in Figure 3.12. We will analyze the three etching experiments illustrated in Figure 3.9 and summarized in Table 3.7 .

During the experiment with $U_{\text {etch }}=2.5 \mathrm{~V}, U_{\text {wafer }}$ starts at $1.65 \mathrm{~V}$ and reduces to 0.59 V. In Figure 3.7(E) the corresponding pores were shown which have a droplet-like geometry. This shape indicates a reduced anisotropy of the etching process. Probably the holes are less selectively moved towards 
the pore tips because the field strength is decreased at low etching potentials. We also conclude that etching at this low current density makes the potential $U_{\text {wafer }}$ change over a large range, which is undesirable and should be avoided by choosing higher current densities $J_{\text {set }}$. Therefore, this etching result is our first indication that higher currents and higher potentials are required to successfully obtain pores.

During the experiment with $U_{\text {etch }}=4 \mathrm{~V}, U_{\text {wafer }}$ starts at $1.96 \mathrm{~V}$ and reduces to $1.7 \mathrm{~V}$, a reduction of only $13 \%$. Under these circumstances the best pores to-date were etched, see Figure 3.7(F). The observed stable growth of pores with the reported diameter in a wafer with a resistivity of $0.76 \pm 0.022 \Omega \mathrm{cm}$ is in good agreement with results in literature [145]. This result shows that if we want to etch pores with smaller diameters and interpore distances, we need to use wafers with a lower resistivity [120, 145, 149]. Unfortunately, purchasing such wafers proved to be challenging as not all desired wafer resistivities were available at suppliers known to us. In other cases, wafers with the desired resistivity were available, but with unusable diameters ${ }^{6}$.

In Figure 3.7(F) we see no change of pore geometry as the pores get deeper, which suggests that the change in potential is not significant enough to distort the etching of the pores. The fact that the change in potential $U_{\text {wafer }}$ is limited is a direct consequence of the elevated set current density $J_{\text {set }}$, since at higher currents the measured hysteresis in the electrolyte is small, see Figure 3.11.

For this reason, etching at higher currents is preferable to etching at low currents. Equation 3.1 shows that to etch pores with equal diameters at higher currents, the critical current density $J_{p s}$ has to be increased proportionally. This can be achieved by changing two different etching parameters:

- Increasing the concentration of HF in the electrolyte results in higher critical current densities [121]. However, the range of usable HF concentrations is limited as it has been reported that obtaining smooth macropores for HF concentrations $>10 \%$ is almost impossible [136]. Furthermore, increasing the concentration of HF in the electrolyte reduces the maximum obtainable pore depth $h$, which is undesirable. Nevertheless, for HF concentrations $<10 \%$ the obtainable pores remain deep with $h>100 \mu \mathrm{m}$ [145]. Hence, increasing the concentration of $\mathrm{HF}$ to values $\leq 10 \%$ is a valid option to obtain higher critical current densities.

\footnotetext{
${ }^{6}$ We would like to acknowledge J. Schilling for the helpful discussion we had by e-mail
} on wafer resistivities and other etching parameters. 
- Increasing the temperature of the electrolyte $[121,140]$ results in higher critical current densities. An added beneficial effect of increasing the electrolyte temperature is that pores will successfully grow at pore tips with a smaller interpore distance $a$.

In the future, these two etching parameters can be optimized to minimize the range of $U_{\text {wafer }}$.

During the experiment with $U_{\text {etch }}=6 \mathrm{~V}, U_{\text {wafer }}$ starts at $3.94 \mathrm{~V}$ and reduces to $3.68 \mathrm{~V}$, a reduction of only $7 \%$. The pore geometry is unchanged as the pores get deeper, see Figures $3.7(\mathrm{~A})$ and (B), similar to the $U_{\text {etch }}=4 \mathrm{~V}$ experiment. In addition, it is seen that pore growth is initiated at each pore tip, which is a promising result. Unfortunately we found indications that for interpore distances of $a<1 \mu \mathrm{m}$ the pores have a tendency to display joining. To obtain stable pores with these interpore distances, the resistivity of the etched wafer must be lower [120], which supports our earlier conclusion based on Figure 3.7(F).

The pores etched with $U_{\text {etch }}=6 \mathrm{~V}$ display a high amount of branching, which is consistent with an applied potential that is too high [120]. Hence, the potential over the wafer needs to be lower than $U_{\text {wafer }}=3.68 \mathrm{~V}$ to etch pores with smooth sidewalls.

Unfortunately, to date we have not been able to reproduce the very deep sub-micrometer pores as reported in literature [150, 151], but the results we obtained indicate that our setup does have the potential to yield such structures. In the next sections we will elaborate on the circumstances that prevented the etching of deep pores. Furthermore, recommendations will be given on how to modify and improve our approach for future experiments.

In terms of etching parameters, we can summarize our current findings as follows:

a) The potential over the wafer $U_{\text {wafer }}$ during etching should be between 1.7 and $1.9 \mathrm{~V}$.

b) During etching the current density should be as high as possible, but well below the critical current density $J_{p s}$.

c) Preferably $J_{p s}$ should be increased, e.g., by using a higher concentration of $\mathrm{HF}$ in the electrolyte.

d) Wafers should be selected with a resistivity less than $0.76 \Omega \mathrm{cm}$. 


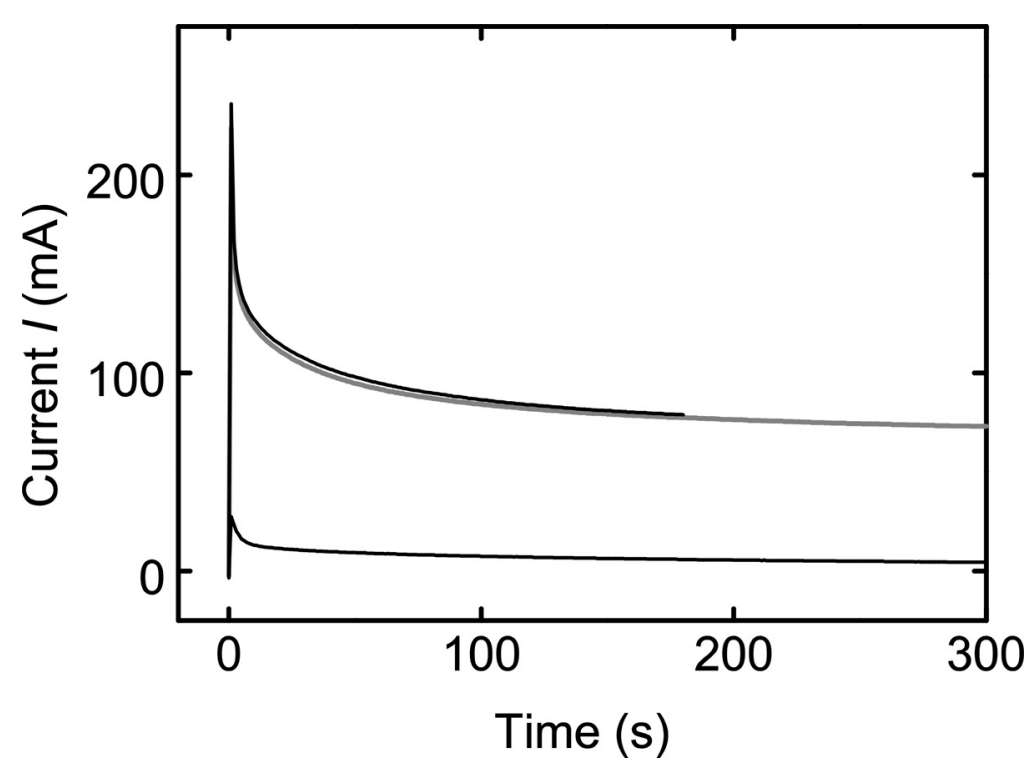

Figure 3.13: Current I at an applied constant potential $U_{\text {elec }}$ as a function of time. No wafer was used during this experiment. The temperature of the electrolyte was approximately $14{ }^{\circ} \mathrm{C}$. The top black line and the grey line show reproducing experiments with $U_{\text {elec }}=2.5 \mathrm{~V}$ and the full wafer holder equipped. The bottom black line is for an experiment with the test wafer holder and $U_{\text {elec }}=1.3 \mathrm{~V}$. In all cases, the current $I$ is decreasing continuously, although in the first seconds very rapidly and then at a reduced rate. This means that the combined resistance of the electrolyte and the electrodes increases in time.

\subsection{Stability and reproducibility}

The most important aspects that prevent the successful etching of deep macropores are the stability and reproducibility of the current $I$ in our etching setup. The first aspect we studied is the stability of the measured current in the situation without wafer. In such experiments, a continuous reduction of the current is observed at constant potentials, see Figure 3.13. This means that the resistance of the electrolyte and electrodes increases in time. A separate stability experiment on an ohmic resistor showed that a constant current was maintained and we concluded that the measured effects are not due to the electronics of the setup. Another aspect we briefly considered as a cause for the increase in electrolyte and electrode resistance is depletion of the HF by, for example, insufficient diffusion in the electrolyte. However, this is ruled out by the fact that consecutive sweeps of $I$ - $U_{\text {elec }}$ curve mea- 
surements on the electrolyte reproduce. As the effects appear to be inherent to the process, they may very well be related to reactions or the adhesion of passivation layers on the electrode surfaces [135].

We assume that during etching experiments the resistance of the electrolyte and electrodes also increases, but here the current is kept constant by an increasing light intensity $L$. This can be observed in Figure 3.8 where the light intensity $L$ increases in time. In etching experiments where the light intensity reached $100 \%$, the increasing resistance could no longer be compensated. Here we observed that the measured current $I$ started to reduce, similar to the stability experiments on the electrolyte. In cases where the resistance in time increases and the current is kept constant by adjusting the light intensity, the potential over the electrolyte and electrodes $U_{\text {elec }}$ gets larger. This causes a reduction in potential over the wafer $U_{\text {wafer }}$ during etching which may cause a change in the geometry of the pores, as illustrated in Figure 3.7. It remains an open question what the underlying mechanism is that causes this effect.

The second aspect we studied is the observed irreproducibility of the current $I$ during experiments on wafers. This irreproducibility occurs both in etching experiments and $I$ - $U$ curve measurements. An example is given in Figure 3.14 where we measured a $I-U$ curve in order to determine the potential at which a wafer of type 2 starts to conduct significantly in the dark. The Figure shows two separate, but equal experiments. The measured current does not reproduce, and there is also a substantial difference in how the $1^{\text {st }}$ and the $2^{\text {nd }}$ sweeps of both experiments relate to each other. The data clearly demonstrate the irreproducibility of the current that we encountered during our experiments on wafers. Figure 3.13 contains an example of two reproducing experiments on the electrolyte only, revealing that this irreproducibility is limited to experiments with a wafer in the etching chamber. The cause of the irreproducibility is currently not understood, but possible explanations include:

- Short circuits due to variable mounting of the wafers in the wafer holders.

- Short circuits due to variable mounting of the wafer holder in the etching chamber.

These mounting issues should be careful screened to solve the irreproducibility of the current $I$.

Other aspects that need to be investigated are the temperature stability of the electrolyte and the homogeneity of the temperature distribution during 


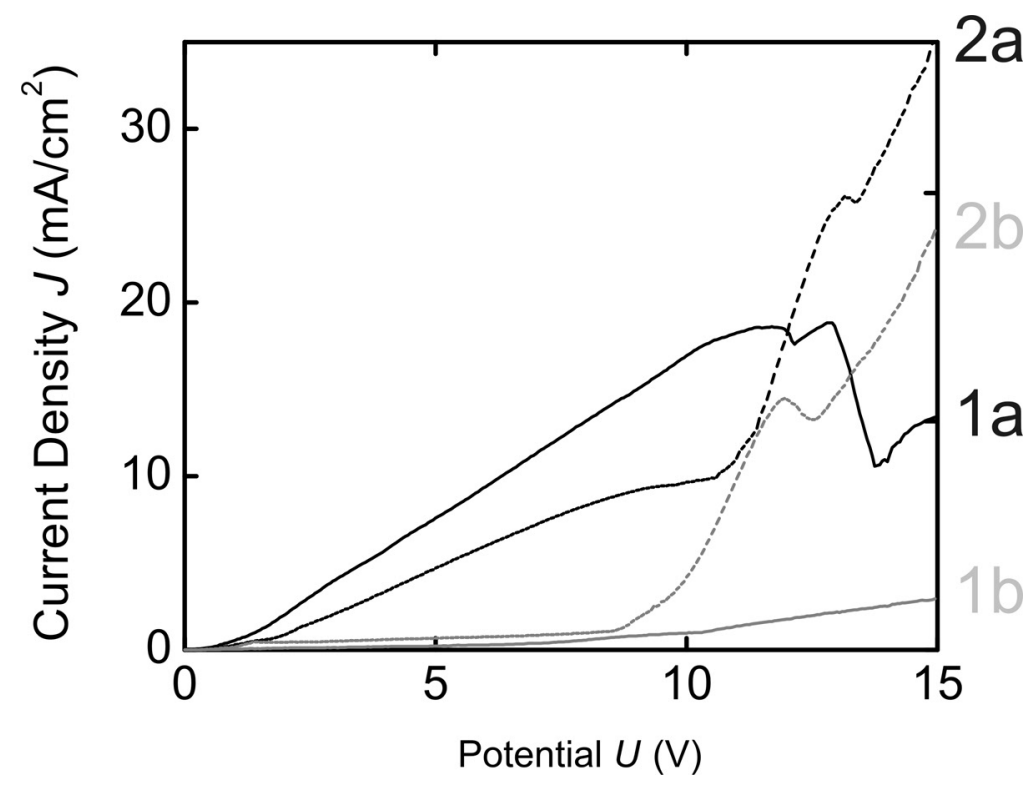

Figure 3.14: $J$ - $U$ curve measured on a wafer of type 2, where an attempt was made to determine the breakthrough potential, i.e., the potential where the wafer starts to conduct without illumination. The test wafer holder was used and the temperature of the electrolyte was approximately $25^{\circ} \mathrm{C}$. Only upward scans are shown. The solid lines are the first sweeps and the dashed lines are the second sweeps. The grey and the black sets represent two separate similar experiments (a) and (b). All current densities for the data set (b, grey) are much lower than those of the data set (a, black). Furthermore, where in the set (b) the second upward scan has a higher current density than the first scan, this is not the case for the set (a). The data demonstrates the irreproducibility of the current density. This was observed for both $J$ - $U$ curves and etching experiments.

etching. A stable and homogeneous temperature is very important for the etching process, as it directly influences the resistance of the electrolyte [136]. Unfortunately, in the current setup, the heat-exchange capability is rather weak. For example, it takes many hours for the system to cool down from 25 to $14{ }^{\circ} \mathrm{C}$. Also, during etching with higher currents we observed that the electrolyte temperature started to increase. No immediate response was observed to the thermostat that tried to compensate and afterwards this resulted in an overshoot on many occasions. Two factors contribute to the poor temperature maintenance. Firstly the heat-exchange tubes mounted at the bottom of both chambers are made from a polymer material, which has limited temperature conductivity. Secondly the magnetic stirrer currently 
can stir the electrolyte only gently, which probably results in a temperature gradient in the electrolyte. These factors need to be addressed in order to etch uniform pores. Another beneficial effect of more vigourous circulation of the electrolyte, preferably in both sides of the etching setup, is that it contributes to the supply of reactants and the removal of etch products and $\mathrm{H}_{2}$ from the wafer. This could result in a better uniformity of the etching process [135], which is also important to obtain high-quality pores.

\subsection{Conclusions}

In this chapter the photo electrochemical etching of macropores in crystalline n-type silicon was described and we introduced the etching setup. The patterning process was described which yielded the inverted pyramidlike pore tips where etching takes place. We showed the best results of etched pores to date, which were analyzed in detail. Calculating the potential over the wafer provides valuable insight in the etching process. We found that the potential over the wafer decreases with time during etching. Conditions were identified at which pores with the desired diameter and interpore distance can be etched: we determined the potential at which the pores should be etched, we showed that etching should be performed at high currents, and we deduced that wafers should be selected with a resistivity lower than $0.76 \Omega \mathrm{cm}$. In particular we discussed that the etching setup is suitable to obtain desired pores after improving the following aspects:

a) The stability of the potential over the electrolyte and electrodes.

b) The mounting of the wafer in the setup.

c) The homogeneity and stability of the temperature of the electrolyte.

d) The circulation of the electrolyte on both sides of the etching setup.

It is expected that after these improvements are implemented the etching process can be better understood and we will be able to reproducibly etch deep, high quality pores with the desired sub-micrometer dimensions. 


\section{Reactive ion etching of deep nanopores}

\subsection{Introduction}

\subsubsection{Fabricating deep nanopores}

The fabrication of spatially periodic nanostructures in silicon receives a great amount of attention in contemporary materials science. Such structures have many interesting applications such as photonic crystals [15], as chemical sensors [152], as a means to alter the wetting of liquids on a surface [153], and as capacitors in high-frequency electronics [154]. The incorporation of such structures on existing silicon integrated circuits is greatly desired, and adapting conventional semiconductor nanofabrication to that end is extensively researched.

A straightforward method to obtain periodic arrays in silicon is the etching of pores in pre-defined patterns and directions. More specifically, to obtain photonic crystals with gaps in the telecommunication windows at wavelengths near 1330 and $1550 \mathrm{~nm}$, the diameter of these pores must be smaller than $500 \mathrm{~nm}$. The pore to pore distances, also referred to as pitch or interpore distance, must be well below $1000 \mathrm{~nm}$. Furthermore the depth to diameter aspect ratio of the pores must be as high as possible to obtain photonic crystals with large volumes. In order to be able to do optical experiments on our structures, we need to obtain pores with high aspect ratios such that the depth of the pores at least exceeds $5 \mu \mathrm{m}$. To avoid unwanted scattering of light, the sidewalls must be as smooth as possible $[155,156]$ and tapering of the pores must be minimized [157]. Finding techniques to make the above-described pores in silicon is extensively researched, see e.g. reference [158], and is the focus of this chapter.

Several techniques have been developed for the formation of arrays of nanopores in silicon. The electrochemical etching of pores is a beautiful ex- 
ample of a fabrication technique for photonic crystals [51]. Although structures with impressive aspect ratios have been obtained, this technique is quite complicated, see chapter 3 , and uses equipment not standard in the Complementary Metal Oxide Semiconductor (CMOS) industry. Therefore photo electrochemical etching is not likely to be incorporated in CMOS semiconductor nanofabrication. Silicon double inversion [159] is another interesting method to realize pores with high aspect ratios. However, this method is rather complex since it requires many preparative steps, including nonCMOS equipment. Since the reported transmittance for wavelengths around $1.5 \mu \mathrm{m}$ is rather low, the technique seems less suited for making photonic structures with band gaps in the telecommunication windows.

Since deep reactive ion etching (DRIE), and more specifically the Bosch process [126], is already used in the semiconductor industry, it is interesting to consider this process for etching nanopores. The use of the Bosch process in the semiconductor industry is exemplified by the fabrication of sloping electrodes [160], antireflection structures [161], and high-speed electronics [154]. Recently the Bosch process has also been used to etch high aspect ratio pores with large diameters of $6 \mu \mathrm{m}$ and interpore distances exceeding $9 \mu \mathrm{m}$ in silicon [162], and pores with diameters of $1 \mu \mathrm{m}$ with interpore distances of around $2.3 \mu \mathrm{m}$ [158]. The two-dimensional photonic crystals formed by these pores have stop bands at wavelengths far outside the telecommunication regions. It is a challenge to reduce the diameters and pitches of the pores to smaller than $1 \mu \mathrm{m}$ in order to obtain gaps around $1550 \mathrm{~nm}$. From literature on one-dimensional trenches with dimensions below $1 \mu \mathrm{m}$, we learn that etching such small features is challenging due to effects like aspect-ratio dependent etching, also known as RIE lag, and that successful etching results require specific processes tuned to the sub-micrometer patterns [125, 163].

An important fabrication aspect is the definition of the required structures by lithography. While e-beam- [118] or laser interference lithography [119] are often used in research, deep UV scan and step lithography is the method of choice in semiconductor industry, since it is fast and allows the patterning of large surfaces. Although the incorporation of deep UV lithography into nanophotonic research seems to be in its infancy, interesting results have already been achieved $[164,165]$.

In this chapter we investigate how to fabricate nanopores with diameters below $500 \mathrm{~nm}$ and pitches well below $1 \mu \mathrm{m}$, with high aspect ratios. The nanopores were pre-defined by a chromium mask patterned using deep UV lithography. 


\subsubsection{Reactive ion etching}

Plasma etching, or reactive ion etching, of silicon is one of the most used tools in the CMOS semiconductor industry and in MEMS fabrication. Exotic examples like the fabrication of a micro-gyroscope [166] and the fabrication of nanometer scaled "rocket tips" for use in an atomic force microscope [167] show the versatility of the technique. Of particular interest to us is the use of reactive ion etching as a tool to make photonic structures in silicon, see e.g. $[61,67,158,164]$. Although this introduction will focus on the reactive ion etching of silicon with $\mathrm{SF}_{6}$ plasmas, it should be noted that many other chemistries are available.

Although the plasma composition is extremely complex, it can be generalized as containing fluoride radicals (F.) and ions $\left(\mathrm{SF}_{x}^{+}\right)$. Both species react with silicon and form volatile $\mathrm{SiF}_{x}$ product compounds, which either participate in further plasma chemistry, or are removed from the system through the vacuum pumps. In this fashion the silicon is etched. Because the fluoride radicals are not influenced by the electric field, they have no preferred direction of movement and diffuse through the whole system. These radicals react with silicon chemically and isotropically, i.e., these reactions have no preferred direction. The ions are forced towards the sample under influence of the electric field. On the sample they enhance the chemical etching of silicon [168] by increasing the etching rate and the anisotropy of the etching process. When the electric field is sufficiently high, the silicon will also be etched by physical sputtering of silicon. Although etching due to ion assisted reactions is mainly directional, sidewall erosion also occurs.

Figure 4.1 shows a schematic overview of an etching setup. The system consists of a capacitively coupled plasma source (CCP) and an inductively coupled plasma source (ICP). The system is connected to vacuum pumps and valves in order to regulate the chamber pressure. By means of entry valves the flow of the gases used for etching can be varied. In addition, helium backside cooling provides the possibility to reduce and stabilize the substrate temperature.

Reactive ion etching can best be explained by first considering what can be referred to as "the traditional method". A traditional system is not provided with an inductively coupled plasma source, which means that in Figure 4.1 the top square is absent. The sample wafer is placed on the capacitively coupled radio frequency driven lower electrode. The capacitively coupled source generates the plasma and simultaneously accelerates the ions towards the sample by an electric field. The molecules in the low pressure $\mathrm{SF}_{6}$ gas are ionized by the oscillating electric field. Electrons gain energy and collide 


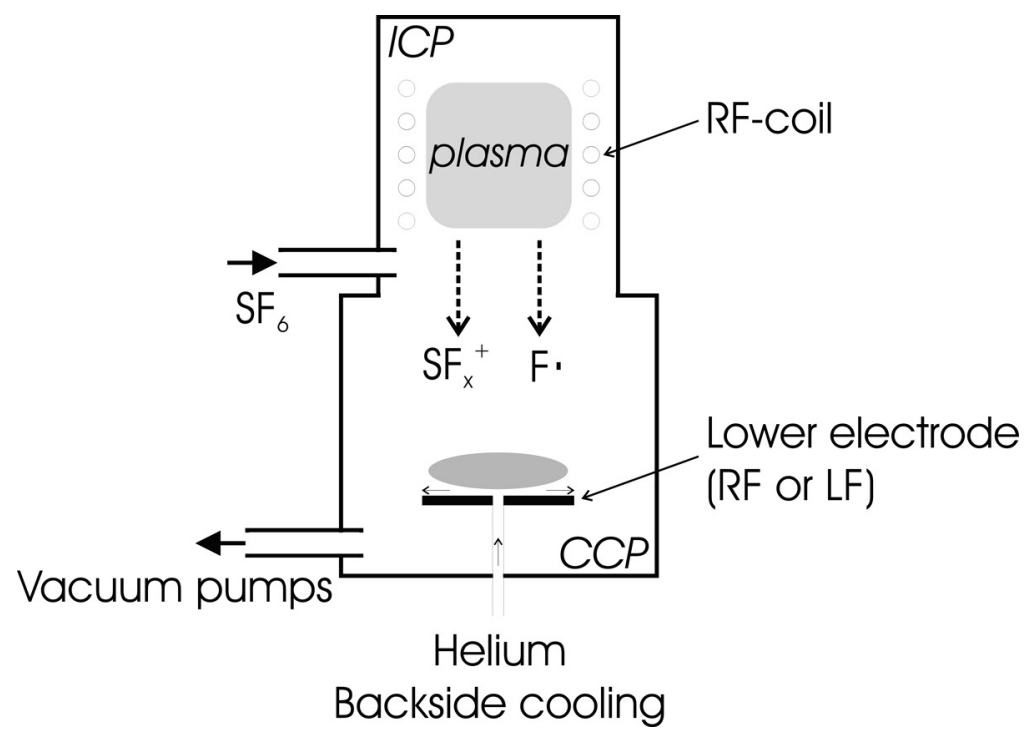

Figure 4.1: Schematic overview of an inductively coupled plasma source etcher. The system is connected to vacuum pumps via a valve to regulate the chamber pressure. Entry valves are used to vary the flow of the etching gasses. The inductively coupled plasma source (ICP) generates the plasma, after which the fluoride radicals diffuse through the chamber. The ionic particles are accelerated towards the lower electrode, which is also the wafer holder, by the capacitively coupled plasma source $(C C P)$. Helium backside regulates the wafer temperature. The sample wafer is shown as the grey ellipsoid.

with atoms and molecules resulting in excitation and dissociation. The resulting plasma composition, consisting of atoms, radical-, and ionic species, is extremely complex [169]. The plasma composition can be influenced by modifying the gas flows, the pressure, and the power on the electrodes. In the case of a traditional reactive ion etching setup the applied power not only influences the composition of the plasma, but also accelerates the ions towards the sample. In such a setup ion density, ion energy, and ion directionality are coupled, and are simultaneously influenced by the capacitively coupled power supply. Therefore these properties cannot be separately controlled, which is an important limitation.

To improve the directionality and etching rates, low pressure and high plasma density equipment was developed. In such systems the plasma is generated by a radio frequency- (ICP) or a microwave source [170], see Figure 4.1. The neutral plasma particles diffuse to the reaction chamber, whereas the ionic particles are accelerated towards the sample by means of the capac- 
itively coupled power supply. This allows for much higher ion densities. The ion to radical ratio on the surface of the sample contributes to a significant increase of the anisotropy of the etching $[124,171]$. Another main advantage of these newer etching systems is that ion density and ion energy are independently controlled. The ion density is controlled by the ICP source and the ion energy is separately controlled by the CCP power supply. The ion angular distribution cannot be separately controlled, but is a resultant of both ion energy and ion density. In order to obtain a small ion angular distribution, the ion density should be low to avoid collisions in the plasma, and the ion energy should be high to improve directionality of the ions.

Two techniques have been developed to allow the fluoride-based etching of deep silicon structures by further reducing sidewall erosion. The idea behind these two techniques is to balance sidewall protection with the etching rate of the structures: the etching rate is determined by the number of ions and radicals in the system, and the sidewall erosion is independently reduced by a protective layer on the sidewalls. Although such a protective layer will also be deposited on the bottom of the etched structures, it will be removed easily by the directional ion bombardment and thus be of virtually no consequence for the etching rate. The first of the two techniques (which will be described) here was introduced by Tachi et al. [172]. By cooling the sample to cryogenic temperatures the reaction probability of fluoride radicals with the sidewalls is strongly reduced, thus reducing the rate of sidewall erosion. Etching of the bottom of the pores is enhanced by the ions. The addition of $\mathrm{O}_{2}$ gas to

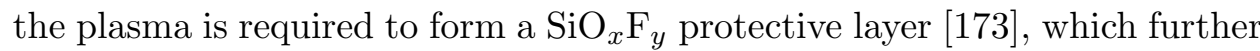
prevents the reaction of fluoride radicals with silicon in the sidewalls. The protective layer thickness is manipulated by modifying the $\mathrm{O}_{2}$ flow. Finetuning of the $\mathrm{O}_{2}$ flow allows the etching of deep structures with sidewalls which have no tapering.

The second technique was introduced by Laermer and Schilp [126], and is commonly known as the "Bosch process". In this process a deposition step and an etching step are continuously alternated. Both of these steps have different chemistries. In the deposition step, also referred to as protection step, a $\mathrm{C}_{4} \mathrm{~F}_{8}$ gas flow is entered into the reaction chamber. From the $\mathrm{C}_{4} \mathrm{~F}_{8}$ plasma a passivating polymer layer that protects the sidewalls from etching is deposited on the sidewalls and bottom of the etched structures. Due to the intensity of the ion bombardment during the etching step, the passivating layer is easily removed from the bottom of the structures and etching proceeds almost unhindered. By carefully manipulating both the etchingand the deposition step deep structures can be obtained. 
Chapter 4. Reactive ion etching of deep nanopores

Table 4.1: Gas flow parameters of the unmodified high aspect ratio structures in silicon recipe. The unit sccm represents a "standard cubic centimeter per minute".

\begin{tabular}{|c|c|c|c|}
\hline & Gas & Flow & Duration \\
\hline Step 1, protection & $\mathrm{C}_{4} \mathrm{~F}_{8}$ & $200 \mathrm{sccm}$ & 1 second \\
\hline Step 2, etching & $\mathrm{SF}_{6}$ & $250 \mathrm{sccm}$ & 3 seconds \\
\hline
\end{tabular}

\subsection{Experimental methods}

In this work $200 \mathrm{~mm}$ single crystal silicon wafers were used (p-type, single side polished $<100\rangle, 1-10 \Omega \mathrm{cm}$ ), which were coated with a $50 \mathrm{~nm}$ thick chromium layer using electron gun evaporation on a Balzers BAK 600. This material was chosen because of its low etch rate in $\mathrm{SF}_{6}$ based reactive ion etch processes [174]. Subsequently, bottom anti reflective coating (BARC, DUV 42), photoresist (PEK 445) and topcoat (Aquatar) were spincoated on the wafers. The resist was patterned using an ASML PAS5500/700 deep UV step and scan system. The pattern was a centered rectangular lattice with a $2 \mathrm{~mm}$ lattice symmetry [117], see Figure 4.2(A), and consisted of many different periodic arrays of holes with a wide range of pitches and a range of hole diameters as shown in Figure 4.2(B). The pitch $a$, or interpore distance, is the shortest distance between two holes, and is shown in Figure 4.2(A). This pattern was chosen as it always cleaves through a set of pores for convenient structural analysis, while it only slightly differs from a square pattern. The angle between the two axes of the primitive cell is $84.5^{\circ}$. The initial pattern density of these structures is the ratio between the etched area and the masked area, and is expressed in percentages. In other experiments a " $\sqrt{2}$ " patterned mask as introduced in chapter 2 was used. In this case the $50 \mathrm{~nm}$ thick chromium layer was patterned using e-beam lithography. After patterning the chromium was etched using chlorine plasma reactive ion etching. Subsequently the photoresist, BARC, and topcoat (where present) were removed.

The patterned wafer was cleaved into pieces of approximately 1.5 by $2.5 \mathrm{~cm}$. These pieces were mounted on $100 \mathrm{~mm}$ p-type silicon dummy wafers. In order to anchor the sample on the dummy wafer and stabilize its temperature, a small droplet of Fomblin ${ }^{\mathrm{TM}}$ vacuum oil was deposited between the sample and the dummy wafer. By applying slight pressure the droplet of oil formed a thin layer between sample and substrate.

The patterned wafers on the dummy wafers were placed in an Adixen Al- 

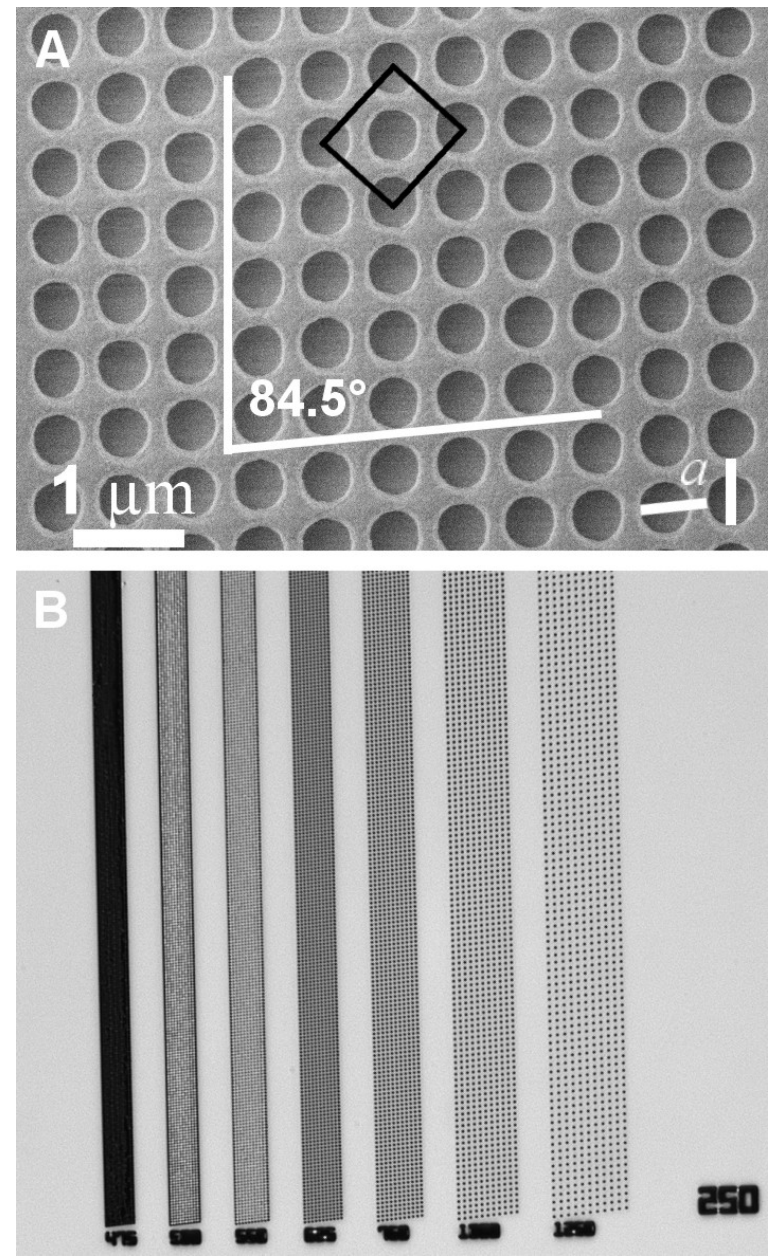

Figure 4.2: A) Scanning electron micrograph of the patterned photoresist. The holes in the photoresist are oriented in a centered rectangular lattice, outlined in black. The pitch $a$ is indicated by the two short white bars. Here the pitch a equals $600 \pm 12 \mathrm{~nm}$ and the diameter is $462 \pm 22.5 \mathrm{~nm}$. The pattern density $\phi=47 \pm 6.4 \%$. Outlined in white are the two axes of the primitive cell, with angle $\varphi=84.5^{\circ}$. B) The periodic patterns of holes are arranged in different sets of diameter-pitch combinations, with a concomitant range of pattern densities. In each set the separate lines have the same specified diameter (here $250 \mathrm{~nm}$ ) and varying pitches (here from $475 \mathrm{~nm}$ to $1250 \mathrm{~nm}$ ). 
catel AMS100SE etcher. The etching parameters we selected are modifications from a Bosch recipe formulated to achieve high aspect ratio structures (HARS) in silicon. The HARS recipe is a two step process, where in one of the steps the pore tips are etched and in the other step the pore walls are laminated with a polymer layer, to reduce sidewall erosion. The gas flow parameters of both steps are shown in Table 1. In our experiments the substrate temperature was regulated by the helium backside cooling (see Figure 4.1 ) and kept at $10^{\circ} \mathrm{C}$. The vacuum throttle valve was completely open. The following parameters were independently controlled during our experiments:

1) The total etching duration was varied between 3 and 12 minutes in order to study the temporal evolution of the etching process.

2) The flow of $\mathrm{SF}_{6}$ was varied from 31 to $200 \mathrm{sccm}$ to find a flow that yields both a high etching rate and minimal sidewall erosion.

3) The flow of $\mathrm{C}_{4} \mathrm{~F}_{8}$ was varied from 160 to $280 \mathrm{sccm}$ to study the effect of increasing the amount of sidewall protection.

4) The duration of the $\mathrm{C}_{4} \mathrm{~F}_{8}$ step was varied from 1 to $4 \mathrm{~s}$ in order to change the amount of sidewall protection.

5) During the etching step the inductively-coupled plasma power (ICP, 13.56 $\mathrm{MHz}$ radiofrequency) was varied from 1000 to $2000 \mathrm{~W}$ to study the effect of changing the density of the reactive species in the plasma.

6) The capacitively coupled plasma power (CCP, around $260 \mathrm{kHz}$ low frequency, $10 \mathrm{~ms}$ on, $90 \mathrm{~ms}$ off) was varied from 20 to $200 \mathrm{~W}$ to study the effect of changing the kinetic energy of the ions.

7) The distance between the CCP driven substrate holder and the ICP source was varied from 110 to $200 \mathrm{~mm}$ in three steps.

Furthermore, the lithographic mask provides a range of pore diameters and pattern densities. When one parameter was varied the other parameters were kept constant at values shown in Table 4.2. In other experiments both CCP power and duration of the $\mathrm{C}_{4} \mathrm{~F}_{8}$ step were simultaneously varied.

After etching the samples were cleaved and the pores near the center of the sample were analyzed using a LEO 1550 high-resolution scanning electron microscope. All pores were imaged from the perpendicular direction $\left(\right.$ tilt $\left.=0^{\circ}\right)$. Figure 4.3 shows a schematic outline of an etched pore and its measured dimensions. The measured depth of the pores is indicated as $h$. All reported diameters $D_{\text {pore }}$ were measured at half of the depth of the 
Table 4.2: Overview of parameter settings which were kept constant as other parameters were varied.

\begin{tabular}{|c|c|}
\hline Parameter & Setting \\
\hline Total etch duration & 6 minutes, unless otherwise stated \\
\hline $\mathrm{SF}_{6}$ flow & $\begin{array}{r}125 \mathrm{sccm} \text { in time-dependence studies, } \\
62 \mathrm{sccm} \text { otherwise }\end{array}$ \\
\hline Duration of $\mathrm{SF}_{6}$ step & 3 seconds \\
\hline $\mathrm{C}_{4} \mathrm{~F}_{8}$ flow & $200 \mathrm{sccm}$ \\
\hline Duration of $\mathrm{C}_{4} \mathrm{~F}_{8}$ step & 1 second \\
\hline ICP power & $1500 \mathrm{~W}$ during both steps \\
\hline CCP power & $200 \mathrm{Wm}$ \\
\hline Substrate to source distance & \\
\hline
\end{tabular}

pores. Similarly, the resist structure and the mask were analyzed by scanning electron microscopy. The measured mask aperture diameter is indicated in Figure 4.3 as $D_{\text {mask }}$. The sidewall erosion was determined by taking the measured diameter of the etched pores $D_{\text {pore }}$, subtracting the mask aperture diameter $D_{\text {mask }}$, and dividing the result by 2 :

$$
\text { sidewall erosion }=\frac{D_{\text {pore }}-D_{\text {mask }}}{2} .
$$

The aspect ratio $A$ is defined as the ratio of $h$ and $D_{\text {pore }}$, see also equation 4.5.

Data of up to ten pores were measured and averaged. The error margins of all measurements are determined by the uncertainty in the measurement and the calibration accuracy of the scanning electron microscope, which is accurate within $2 \%$. The error margins in the erosion is given by one half of the errors of $D_{\text {mask }}$ and $D_{\text {pore }}$ combined.

\subsection{Results and discussion}

\subsubsection{Deep UV Lithography}

Since the lithography defines the location and diameter of the subsequently etched pores, we will briefly analyze the photoresist and chromium mask structures. Figure 4.2(B) is an optical microscopy image of arrays of holes with one particular specified diameter $(250 \mathrm{~nm})$, and pitches varying between 


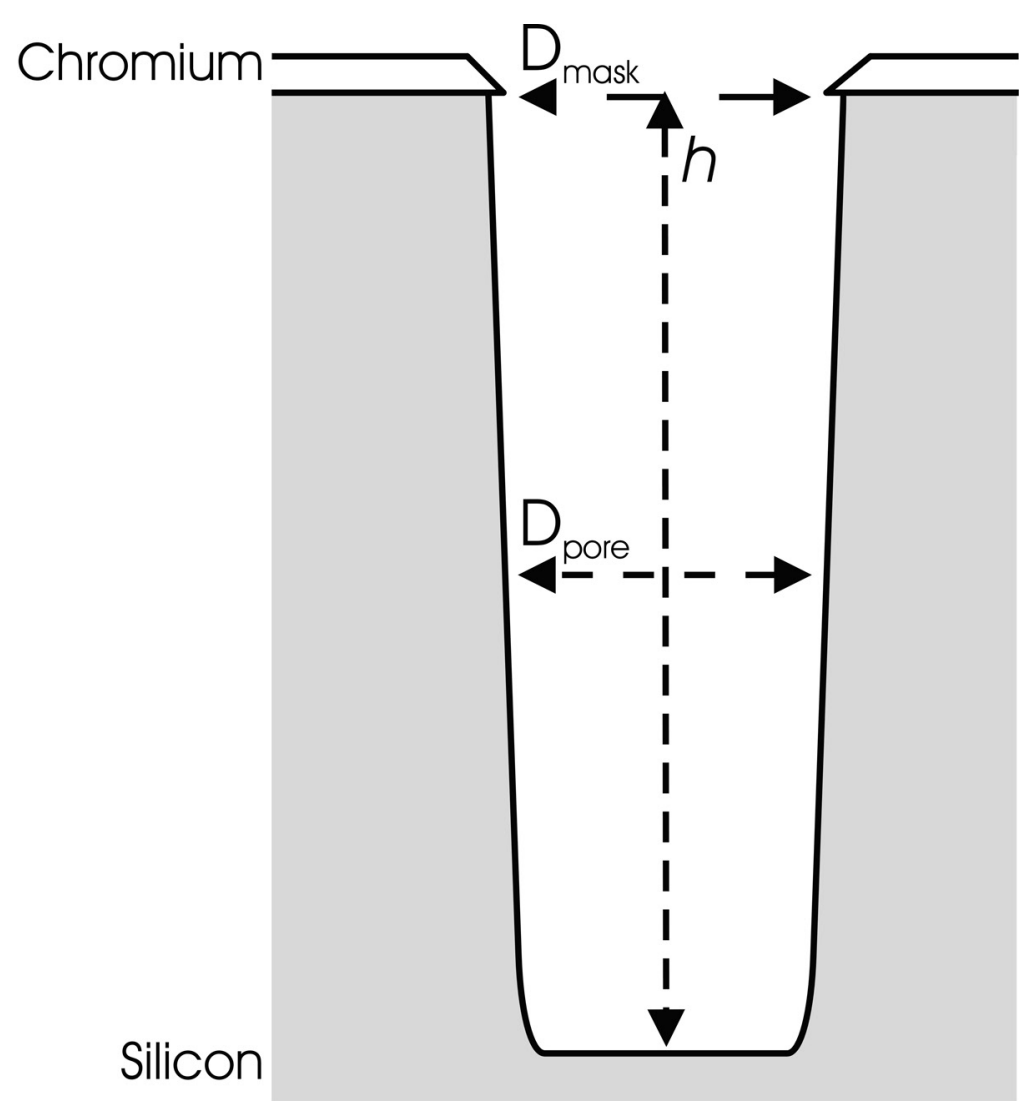

Figure 4.3: Schematic overview of a pore etched in silicon through a chromium mask. Indicated are both materials, the measured depth of the pores $h$, the measured mask aperture diameter $D_{\text {mask }}$, and the measured diameter of the pores $D_{\text {pore }}$ at half the pore depth. The sidewall erosion is defined as $\frac{1}{2}$ times the difference between the measured diameter of the etched pores $D_{\text {pore }}$ and the mask aperture diameter $D_{\text {mask }}$. The aspect ratio $A$ is defined as $h$ over $D_{\text {pore }}$.

475 and $1250 \mathrm{~nm}$. The length of the arrays of holes extend over more than $15 \mathrm{~mm}$, that is more than 15000 pores. Such large structures are made without stitching, which is an advantage of deep UV lithography over e-beam lithography. Moreover, the simultaneous lithography of different diameterpitch combinations is an advantage over laser interference lithography.

Figure 4.2(A) shows a high resolution scanning electron microscopy image of a photoresist pattern after step and scan processing. The hole diameter is $D=462 \mathrm{~nm}$ and the pitch is $a=600 \mathrm{~nm}$. Data from many such images were analyzed and results are summarized in Figure 4.4 for one particular 
pattern density. Figure 4.4 shows the measured hole diameters versus pitch, both for the patterned photoresist, and the subsequent chromium mask. The data reveal that the holes in the chromium layer are tapered, as shown schematically in the inset, since the diameters at the top of the layer are systematically larger than at the bottom. The effective mask is defined as the aperture bounded by the bottom of the hole. We see that the effective mask aperture diameter is significantly smaller than the diameter defined by the circles in the photoresist pattern. To determine the pattern density of the diameter-pitch combinations shown in Figure 4.4, a line was fitted through the effective mask aperture diameter and the origin, using linear regression with a confidence of $95 \%$. As shown in appendix A, the slope of this line relates to the pattern density, yielding in this case a value of $\phi=25.9 \pm 0.77 \%$. Since the reactive ion etching of a pattern is influenced by the pattern density [175], it is important to determine this parameter. Furthermore, since the pattern densities of the diameter-pitch combinations shown are equal, these combinations are suitable for further analysis of etching experiments where other processing parameters were varied.

\subsubsection{Nanopores in silicon}

Figure 4.5 gives a pictorial overview how increasing the etching duration and reducing the flow of $\mathrm{SF}_{6}$ influences the etching result. From scanning electron micrographs like the ones shown in Figure 4.5, the depth $h$ and the diameters of the pores $D_{\text {pore }}$ were measured. The apparent damage on several structures is due to the cleaving of the sample, but the deeply etched pores are well visible. The roughness observed in the first micrometers near the surface of the wafer is a sequence of evenly spaced rims caused by the cyclic nature of the Bosch etching process. These rims are commonly referred to as scallop $[161,167]$ and can cause unwanted scattering of light. Figure 4.5(A) shows a set of pores etched for 3 minutes with $125 \mathrm{sccm} \mathrm{SF}_{6}$. These pores have almost no tapering, but with a depth of $h=2.85 \mu \mathrm{m}$ they are not deep enough for our purposes. To fabricate deeper pores we increased the etching duration. Figure 4.5(B) shows pores etched for 12 minutes with the same mask. These pores are indeed deeper and have a depth of $h=7.9 \mu \mathrm{m}$. Unfortunately increasing the etching duration not only influences the depth of the pores, but also increases sidewall erosion. Therefore the diameter of the pores increases so much that the pores start to break down. The diameter increased from $D_{\text {pore }}=421 \mathrm{~nm}$ after 3 minutes of etching to $D_{\text {pore }}=574 \mathrm{~nm}$ after 12 minutes of etching. The tapering of these pores is estimated to be about $0.5^{\circ}$, which is slightly more compared to the 3 minutes experiment. 


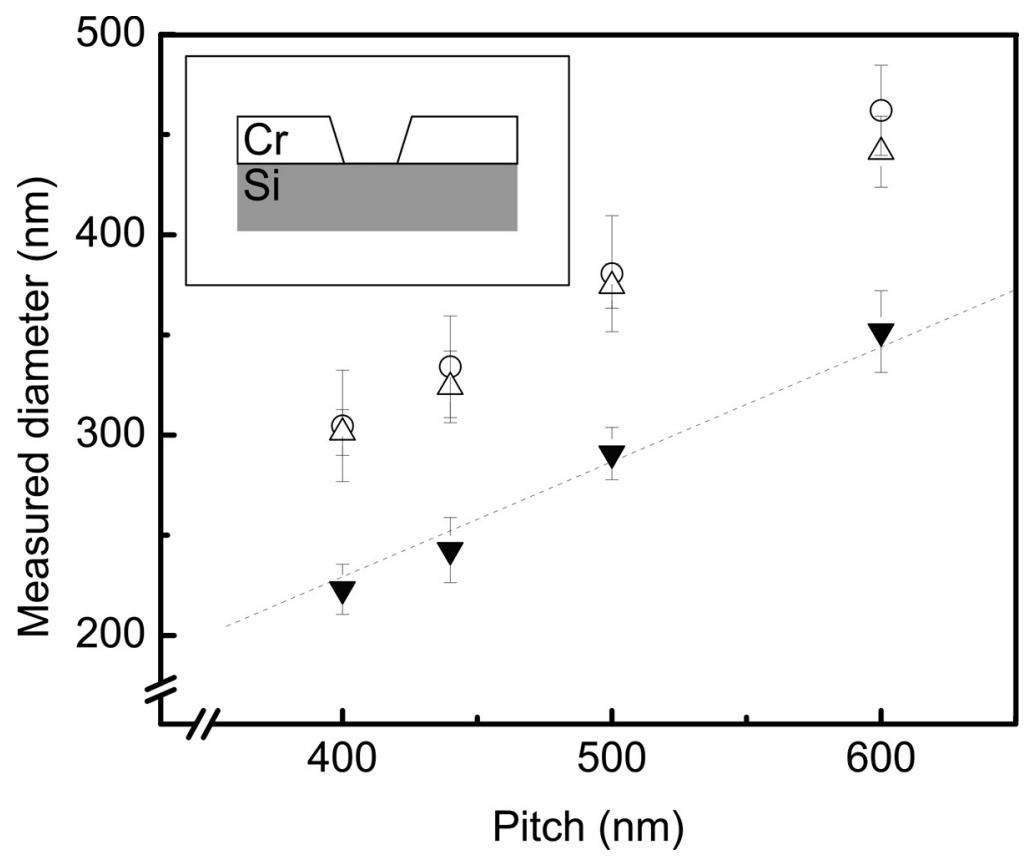

Figure 4.4: The measured diameter of holes versus the pitch in the mask in the photoresist (circles) and in the chromium layer. As shown schematically in the inset the holes in the chromium layer are tapered. The upward triangles denote the diameter of the holes at the top of the chromium layer. The filled downward triangles are the hole diameters at the bottom of the chromium layer and are taken as the effective hole diameters. The dotted line is a fit from which the pattern density is found to be $\phi=25.9 \pm 0.77 \%$.

Figure 4.5(C) shows another set of pores etched for 12 minutes with $125 \mathrm{sccm}$ $\mathrm{SF}_{6}$. In this case the sidewall erosion caused the pores to overlap and break down. To limit the sidewall erosion the flow of $\mathrm{SF}_{6}$ was reduced. Figure 4.5(D) shows pores etched with the same mask, but with $62 \mathrm{sccm} \mathrm{SF}_{6}$. The pores have a diameter of $D_{\text {pore }}=351 \mathrm{~nm}$ and individual pores no longer overlap, which shows that reducing the flow of $\mathrm{SF}_{6}$ does indeed yield pores with less sidewall erosion. While the depth of these pores is slightly less than the pores etched with $125 \mathrm{sccm} \mathrm{SF}_{6}$, the aspect ratio of these pores is as high as 16. The pores shown in Figures 4.5(B) and (D) have smooth sidewalls, apart from the upper 1 to 2 micrometers. 


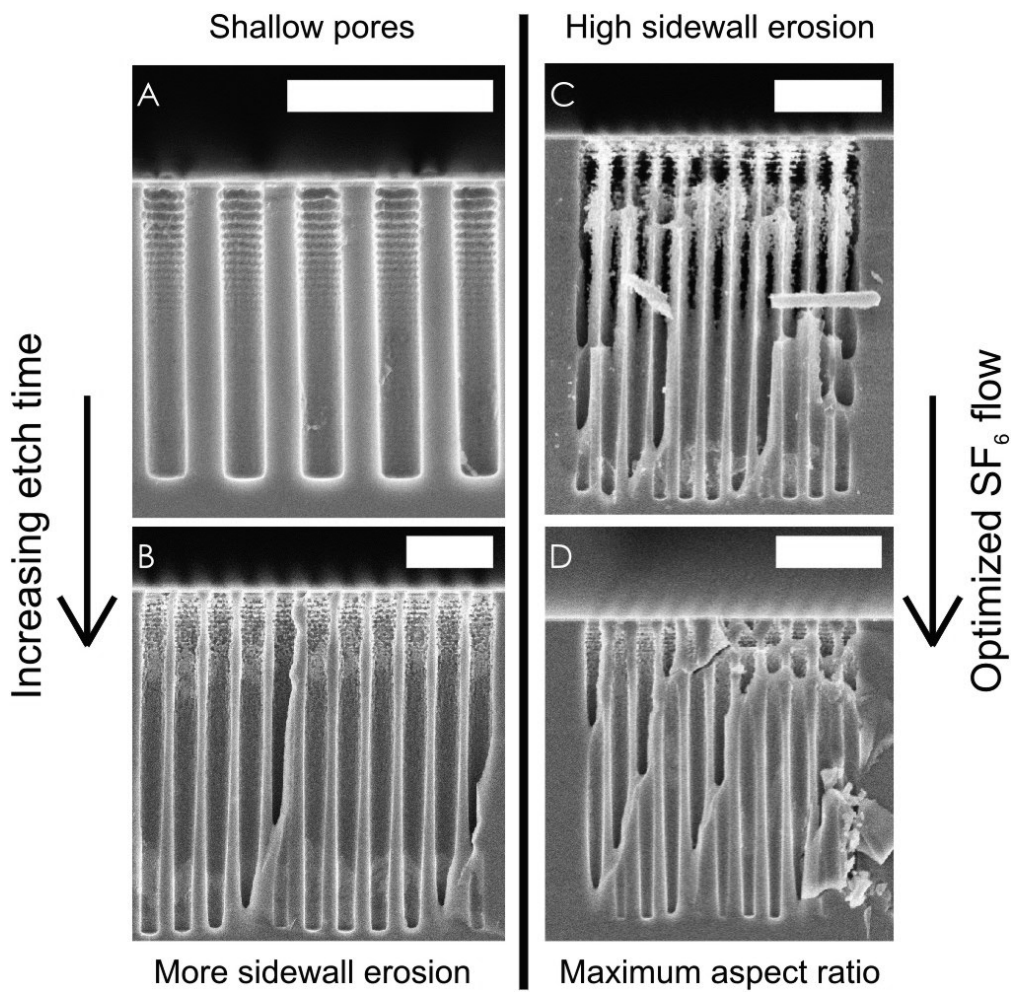

Figure 4.5: Scanning electron micrograph for a representative set of cleaved samples etched under different conditions. In all images the scale bar equals $2 \mu \mathrm{m}$. A) Pores etched for 3 minutes with $125 \mathrm{sccm} S F_{6}$. The depth of these pores is $h=2.85 \pm$ $0.066 \mu \mathrm{m}$ and their diameter is $D_{\text {pore }}=421 \pm 17.5 \mathrm{~nm}$. B) Pores etched with the same mask for 12 minutes. These pores have a depth of $h=7.9 \pm 0.16 \mu \mathrm{m}$. Their diameter is $D_{\text {pore }}=574 \pm 29 \mathrm{~nm}$. C) Pores etched for 12 minutes with $125 \mathrm{sccm}$ of $S_{6}$. The sidewall erosion was significant, causing the pores to break down. D) Pores etched with the same mask as $C$ ), but with an optimized flow of $S_{6}$ of 62 sccm. These pores have the least sidewall erosion resulting in the highest aspect ratio of $16 \pm 1.4$, with a pore diameter of $D_{\text {pore }}=351 \pm 21 \mathrm{~nm}$ and a pitch of $a=484 \pm 9.7 \mathrm{~nm}$. We estimate the tapering to be about $0.5^{\circ}$. 


\subsubsection{Exploring seven parameters of the etching process}

In order to improve the pore depth, decrease the sidewall erosion and decrease the tapering, seven process parameters were systematically varied. The effects of varying these parameters is discussed in the following sections.

\section{i) Total etch duration}

To study any etching process it is important to analyze the evolution of that process as time progresses. The measured diameter, depth, and aspect ratio are plotted as a function of total etching duration in Figure 4.6. Data are shown for two different diameter-pitch combinations, with different initial pattern densities $\phi$ of 5.59 and $11.2 \%$.

Figure 4.6(A) shows that the depth of the pores increases with etching duration, and the increase levels off slightly as the aspect ratio increases. This means that the etching rate decreases with increasing aspect ratios, which is consistent with an aspect ratio dependent etching process [163]. The dashed lines show a model that will be discussed in more detail in section 4.4.

From Figure 4.6(B) it is apparent that the diameter of the pores steadily increases in time; despite the protection of the sidewall, some sidewall erosion occurs. To determine the rate of sidewall erosion per unit time a straight line was fit to the data. One half of the slope of such a line represents the rate of sidewall erosion in nm per minute. The linear relation between measured diameter and etching duration shows that the rate of sidewall erosion is constant in time, and not aspect ratio dependent. Therefore an important conclusion is that the aspect ratios that can be achieved with this technique are limited, since the depth increases slower and slower with time, while the diameter increases linearly with time.

Figure 4.6(C) confirms that although initially the aspect ratio increases rapidly, the increase in aspect ratio levels off as etching progresses. At etching durations below 12 minutes we observe that narrower pores have higher aspect ratios, similar to results reported for large one-dimensional trenches $[160,176]$. At etching durations exceeding $t=12$ minutes the aspect ratio appears to reach a maximum. The dashed lines are derived from the same model as in Figure 4.6(A).

\section{ii) Etchant flow}

To study the effect of the flow of $\mathrm{SF}_{6}$, we measured the diameters $D_{\text {pore }}$ and the pore depths $h$ of the four diameter-pitch combinations shown in Figure 


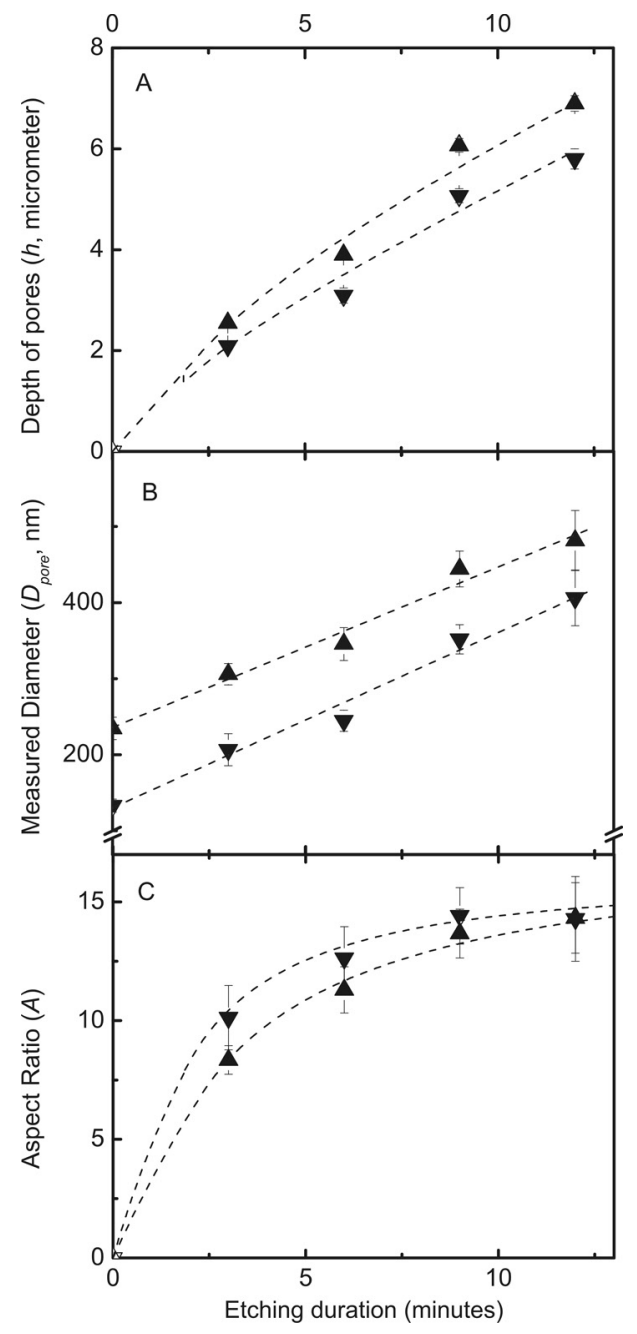

Figure 4.6: Pore dimensions for 2 different pattern densities as a function of etching duration, etched with a flow of $S_{6}$ of $125 \mathrm{sccm}$. The upward triangles indicate mask aperture diameter $D_{\text {mask }}=235 \pm 15 \mathrm{~nm}$ and pitch $a=625 \pm 13 \mathrm{~nm}$, corresponding to a density of $\phi=11.2 \pm 1.8 \%$. The downward triangles indicate mask aperture diameter $D_{\text {mask }}=133 \pm 8.6 \mathrm{~nm}$ and pitch $a=500 \pm 10 \mathrm{~nm}$, corresponding to a density of $\phi=5.6 \pm 0.94 \%$. A) The pore depths increase as etching time progresses, but levels off. The dashed lines show the results for the fits of a model that explains the data (equation 4.10). B) The diameter of the pores increases steadily in time. The dashed lines are linear fits. The slope for pattern density $\phi=5.59 \%$ is $\alpha=23 \pm 1.7 \mathrm{~nm} / \mathrm{min}$ and the slope for pattern density $\phi=11.1 \%$ is $\alpha=21 \pm 1.6 \mathrm{~nm} / \mathrm{min}$. C) The initial aspect ratio rapidly increases, but as the etching progresses the aspect ratio levels off. After $t=12$ minutes, the aspect ratio no longer increases. The dashed lines are derived from the same model as in A), with $A$ equals $h$ over $D_{\text {pore }}$. 
4.4. The pores were etched for 6 minutes with different flows of $\mathrm{SF}_{6}$. All measured values were obtained from images similar to Figure 4.5. For two of the diameter-pitch combinations, the average etching rates are shown versus the flow of $\mathrm{SF}_{6}$ in Figure 4.7(A). The average etching rate was calculated by dividing the measured depth of the pores $h$ by the total etching duration. Figure 4.7(A) illustrates that the etching rate is highest at $200 \mathrm{sccm}$. Pores etched with $125 \mathrm{sccm}$ and $62 \mathrm{sccm} \mathrm{SF}_{6}$ show a reduced etching rate. At 31 sccm the etching rate dramatically decreases, and the obtained aspect ratio of the pores is reduced. Obviously, at $0 \mathrm{sccm}$ no etching and sidewall erosion occurs.

From the measured diameters we obtained the amount of sidewall erosion, see Figure 4.7(B). For all flows of $\mathrm{SF}_{6}$ sidewall erosion occurred during etching, and at higher flows of $\mathrm{SF}_{6}$ the amount of sidewall erosion is larger. The larger sidewall erosion is due to the increased exposure of the sidewalls to the etching plasma, whereas the amount of sidewall protection stays the same. In addition, a higher flow of $\mathrm{SF}_{6}$ results in a higher chamber pressure [177], which causes reduced directionality of the plasma due to collisional scattering of ions [178] and therefore more sidewall erosion. By combining the results for the sidewall erosion and the etching rate, we conclude that an $\mathrm{SF}_{6}$ flow of $62 \mathrm{sccm}$ is optimal, since the amount of sidewall erosion is fairly low while maintaining a sizeable etching rate.

\section{iii) Flow in the protection step}

The etching experiments described so far show significant sidewall erosion. This suggests that the overall etching process has an isotropic character, because of insufficient sidewall protection. Therefore, altering the deposition step such that the polymer layer thickness increases is expected to have a favorable effect on the sidewall erosion. Similar to the $\mathrm{SiO}_{x} \mathrm{~F}_{y}$ passivation in $\mathrm{SF}_{6} / \mathrm{O}_{2}$ cryogenic etching processes [177], too little protection will result in negatively tapered structures, whereas too much protection results in positively tapered structures. By applying a correct amount of protection, structures without tapering can be etched. Increasing the deposition of $\mathrm{C}_{4} \mathrm{~F}_{8}$ on the sidewalls can be achieved by either increasing the duration of the deposition step, or increasing the flow of $\mathrm{C}_{4} \mathrm{~F}_{8}$ in that step [179]. When increasing the flow of $\mathrm{C}_{4} \mathrm{~F}_{8}$ the pressure in the system also increases which reduces the polymer deposition rates [180]. Ultimately a regime is reached where, although the flow of $\mathrm{C}_{4} \mathrm{~F}_{8}$ is increased, the polymer layer gets thinner.

We raised the flow of $\mathrm{C}_{4} \mathrm{~F}_{8}$ from $160 \mathrm{sccm}$ to $280 \mathrm{sccm}$ and measured the average etching rate, sidewall erosion, and tapering for two sets of pores, 


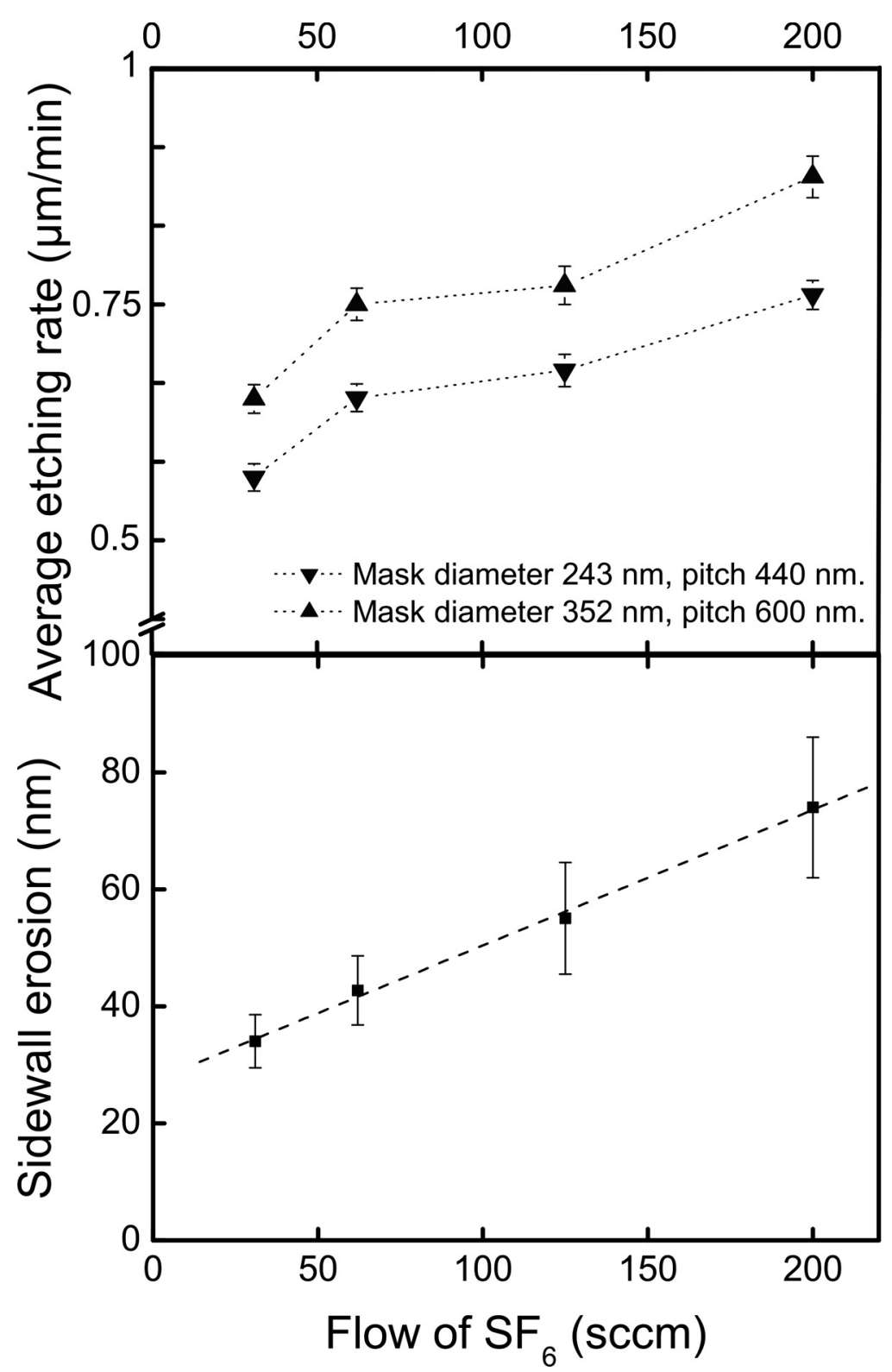

Figure 4.7: A) Average etching rate versus the flow of $S F_{6}$ for two of the diameterpitch combinations. The average etching rate was calculated by dividing the measured depth of the pores $h$ by the total etching duration. The dotted lines are guides to the eye. B) Sidewall erosion of pores after etching for 6 minutes as a function of the flow of $\mathrm{SF}_{6}$. The sidewall erosion increases with flow. 
see Figure 4.8. When the flow of $\mathrm{C}_{4} \mathrm{~F}_{8}$ is increased, the average etching rate is not influenced for $D_{\text {mask }}$ equals $306 \mathrm{~nm}$, and slightly reduced for $D_{\text {mask }}$ equals $237 \mathrm{~nm}$, see Figure $4.8(\mathrm{~A})$. The slight decrease is probably caused by the following effect: the $\mathrm{C}_{4} \mathrm{~F}_{8}$ step not only deposits polymer, but also contributes to the etching of the pores, see below. If in the $\mathrm{C}_{4} \mathrm{~F}_{8}$ step polymer deposition and pore etching do not change equally with increasing flow, i.e. the etching rate increases slower than the deposition rate, this will cause a slightly decreased average etching rate, as observed.

Figure $4.8(\mathrm{~B})$ shows the sidewall erosion versus the flow of $\mathrm{C}_{4} \mathrm{~F}_{8}$. As expected the sidewall erosion decreases with higher flows, although only by approximately $10 \mathrm{~nm}$, which is less than $10 \%$ of the mask aperture radius. Nevertheless, this result does confirm that sidewall protection occurs as a result of the $\mathrm{C}_{4} \mathrm{~F}_{8}$ step, and that modifying the $\mathrm{C}_{4} \mathrm{~F}_{8}$ step can be used as a tool to control pore geometry. In the next section we will study whether increasing the duration of the protection step results in a larger reduction of the sidewall erosion. Our data do not indicate a significant influence of the flow of $\mathrm{C}_{4} \mathrm{~F}_{8}$ on the tapering of the pores, see Figure $4.8(\mathrm{C})$, which is due to the limited effect on the sidewall protection.

\section{iv) Duration of the protection step}

Since altering the flow of $\mathrm{C}_{4} \mathrm{~F}_{8}$ during the deposition step only reduced the sidewall erosion by approximately $10 \mathrm{~nm}$, we decided to increase the thickness of the polymer layer by increasing the duration of the deposition step from 1 to 3 seconds at a flow of $\mathrm{C}_{4} \mathrm{~F}_{8}$ of $200 \mathrm{sccm}$. In order to make sure that the total number of etching and deposition steps remained the same, the total etching duration was increased to compensate for the longer duration of the deposition step. Figure 4.9 shows the results of these experiments. When the duration of the protection step increases, the average etching rate also increases, see Figure 4.9(A). Since the etching step is not modified, this means that the deposition step also contributes to the etching of the pores. Obviously at the bottom of the pores there is competition between depositing of polymer, and removal of polymer by the $\mathrm{C}_{4} \mathrm{~F}_{8}$ plasma itself. Our observation that the $\mathrm{C}_{4} \mathrm{~F}_{8}$ step contributes to the etching of the pores suggests that during this step there is no net deposition of polymer on the bottom of the pores. This leads to the conclusion that when the system enters an etching step, the $\mathrm{SF}_{6}$ plasma does not have to etch through a layer of polymer at the bottom of the pore before reaching the silicon, which is optimal for etching [181].

Figure 4.9(B) shows that increasing the duration of the deposition step is 


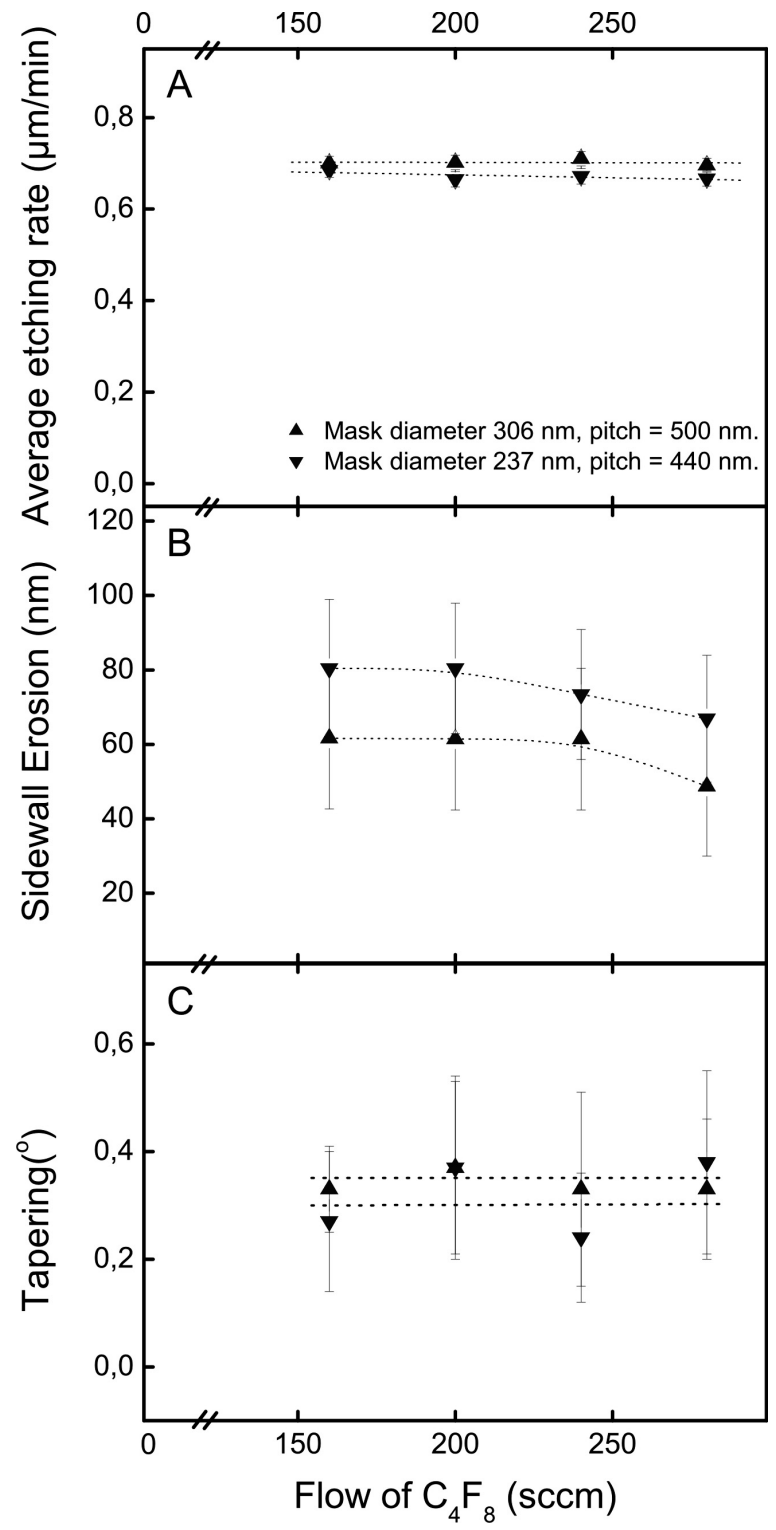

Figure 4.8: Average etching rate, sidewall erosion, and tapering of etched pores versus the flow of $\mathrm{C}_{4} \mathrm{~F}_{8}$ applied during the deposition step. All dotted lines are guides to the eye. A) The average etching rate is not significantly influenced by the flow of $\mathrm{C}_{4} \mathrm{~F}_{8}$. B) The sidewall erosion is reduced with increasing flow. C) The tapering of the etched pores is about $0.3^{\circ}$ for all flows of $C_{4} F_{8}$. There is no significant effect of the flow on the observed tapering. 
a powerful tool to decrease the sidewall erosion of the pores. The data show that when the duration of the deposition step is increased to $3 \mathrm{~s}$, no sidewall erosion is observed. Unfortunately, at these step durations of $3 \mathrm{~s}$, the pores show increased tapering, see $4.9(\mathrm{C})$. At durations of 1 and $2 \mathrm{~s}$, tapering of the pores was limited to about $0.3^{\circ}$, but at $t=3 \mathrm{~s}$ the tapering increased to more than $0.6^{\circ}$. Therefore we argue that increasing the duration of the protection step to longer then $3 \mathrm{~s}$ is not useful per se and should only be used when the tapering remains below acceptable values.

\section{v) Influence of the inductively coupled plasma source}

To study the influence of the ICP power on our etching process, we performed a series of experiments in which the ICP power during the etching step was chosen varied from $1000 \mathrm{~W}$ to $2000 \mathrm{~W}$. During the deposition step the ICP power was kept at $1500 \mathrm{~W}$ to make sure that the amount of sidewall protection was equal for all experiments. Figure 4.10(A) shows that increasing the ICP power leads to a slightly increased average etching rate. The average etching rate versus the ICP power in our experiments agrees qualitatively with results measured when etching full wafers as described in [177]. The increased average etching rate at higher ICP powers is explained by an increased number of ions in the plasma, whereas the number of fluoride radicals is expected to decrease only slightly when increasing the ICP power from 1000 to $2000 \mathrm{~W}$ [182]. We see that the rate of sidewall erosion is unchanged, see Figure 4.10(B). Apparently the changed plasma composition has no overall effect on the etching of the sidewalls. Figure 4.10(C) shows that the tapering of the pores is larger when the ICP power is increased. We explain this by the lower energy of the ions in the plasma, which results in less directionality of the plasma and therefore more tapering. This effect was also found in simulations, see e.g. [183]. We conclude that modifying the ICP power in the etching step does not improve our nanopores: the sidewall erosion is not reduced, more tapering occurs, and the etching rate hardly increases.

\section{vi) Capacitively coupled plasma source}

To study the influence of the CCP power on our etching process, we performed a series of experiments in which the CCP power was varied. Due to technical reasons the matching of the CCP source during the deposition steps is poor, which means that not all of the power of the source is coupled to the plasma. Therefore the effective CCP power during a deposition step is less than the set value. Typically when the CCP power is set to $80 \mathrm{~W}$, 


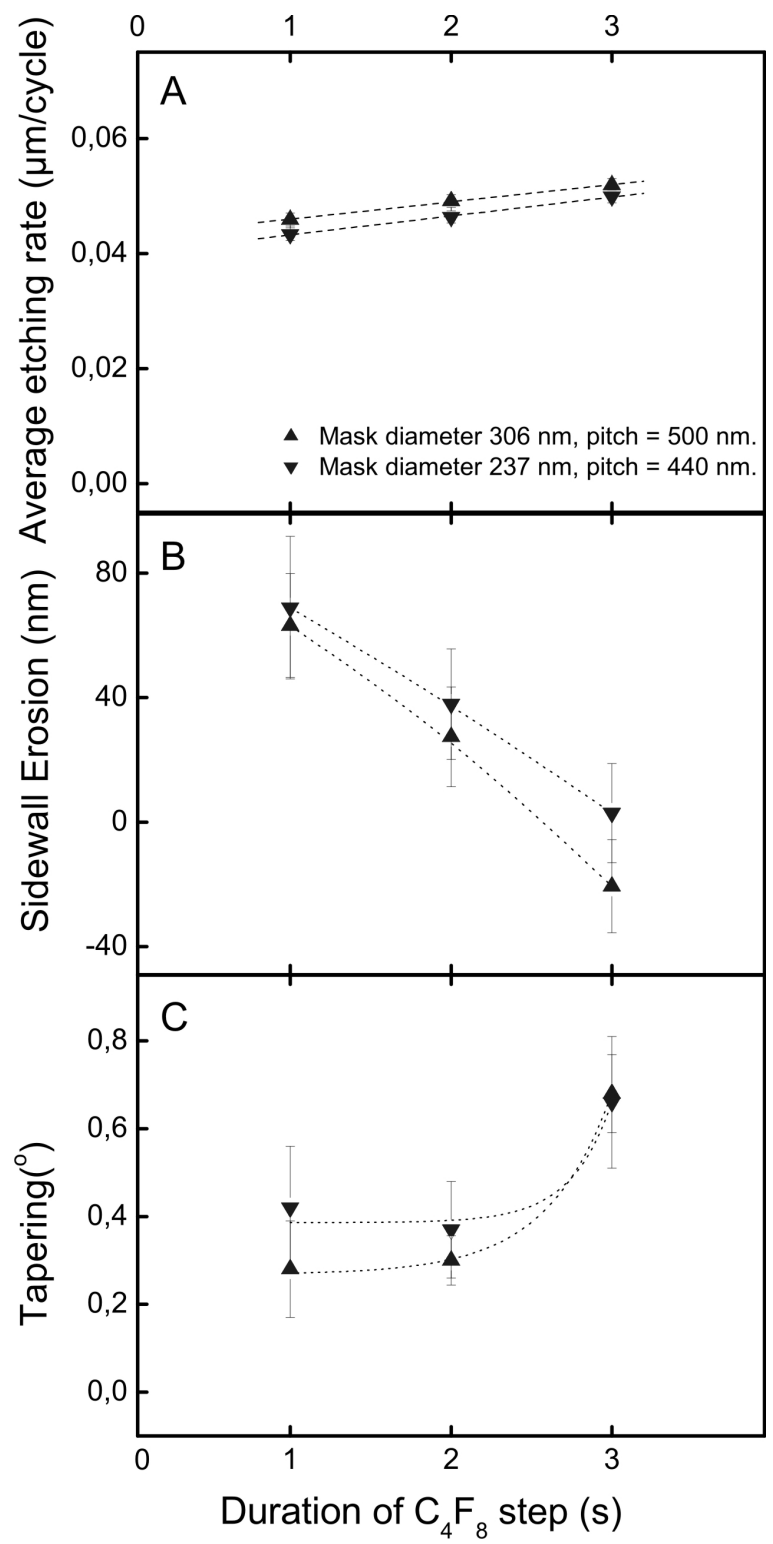

Figure 4.9: Average etching rate, sidewall erosion, and tapering of etched pores versus the duration of the deposition step. All dotted lines are guides to the eye. A) The average etching rate increases with longer step durations, which indicates that $C_{4} F_{8}$ not only protects, but also etches. B) The sidewall erosion is significantly reduced with longer durations. At $t=3 \mathrm{~s}$, no sidewall erosion is observed at half of the depth of the pores with respect to the mask aperture diameter $D_{\text {mask }} . C$ ) The tapering of the etched pores is low for durations up to $2 \mathrm{~s}$, but at $t=3 \mathrm{~s}$ the tapering exceeds $0.6^{\circ}$. 


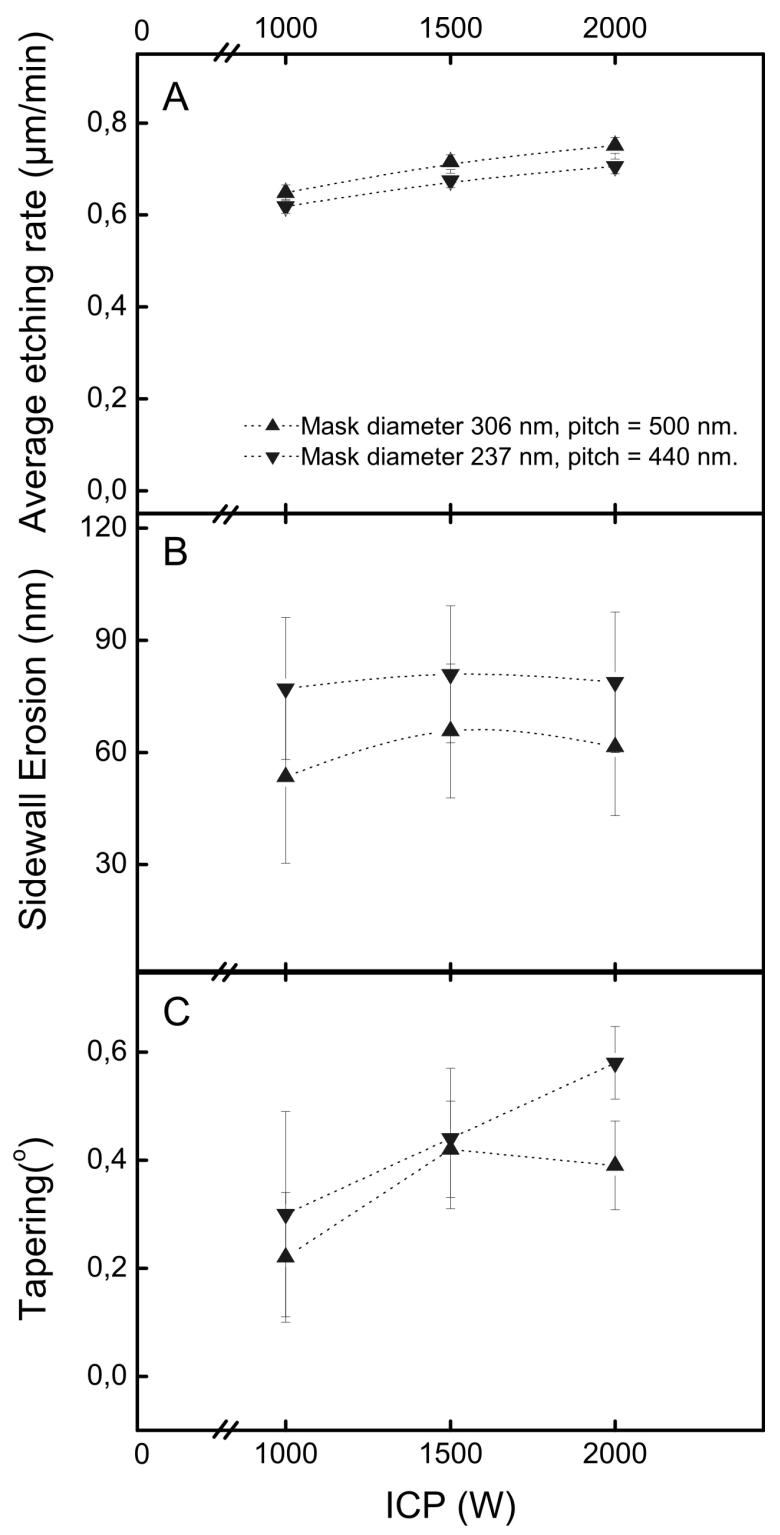

Figure 4.10: Average etching rate, sidewall erosion, and tapering of etched pores versus the ICP power varied during the etching step. All dotted lines are guides to the eye. A) The average etching rate is slightly increased with higher ICP powers. This is due to an increase of the amount of reactive species in the plasma. B) When increasing the ICP from 1000 to $2000 \mathrm{~W}$ the effect on the sidewall erosion was not significant. C) Increasing the ICP power during the etching step leads to pores with more tapering. This is due to a lower directionality of the plasma. 
the power to the plasma is about $60 \pm 10 \mathrm{~W}$. In both the etching and the deposition steps, the ICP value is kept constant. Therefore, with increasing CCP power the energy of the ions increases, while the ion current density remains constant. With increasing ion energy, the directionality of the plasma increases. This means that during the etching step more ions arrive at the bottom of the pores, and with a higher kinetic energy. However, as shown in Figure 4.11(A) the etching rate is not influenced by the CCP power, and consequently not influenced by the kinetic energy of ions. This result shows that:

a) energy dependent physical sputtering of silicon by the ions is negligible,

b) the increased number of ions due to the improved directionality is not significant enough to have an effect on the average etching rate, and

c) the enhancement of the etching by fluoride radicals is not influenced by the energy of the ions assisting the process. It remains an open question why the average etching rate at a CCP power of $40 \mathrm{~W}$ is slightly higher.

Figure 4.11(B) shows that the sidewall erosion increases at higher CCP powers. We think that two factors contribute to this result. Firstly, the ions which collide with the sidewalls have more energy and remove more polymer per ion. This is a more significant effect than that of the reduced number of ions hitting the sidewalls because of the improved directionality. Overall this results in less sidewall protection, and more erosion occurs. Secondly, because of the higher CCP power, the ion energy in the deposition step is increased. This leads to more in-situ sputtering of the deposited polymer by the ions [181], resulting in a thinner polymer layer on the sidewalls. Hence the sidewalls are less protected during the subsequent etching step, resulting in a higher rate of sidewall erosion.

Figure 4.11(C) shows that the tapering of the pores is strongly reduced at high CCP powers. This is due to the increased directionality of the plasma and the lower amount of sidewall protection. Because of the strong effect on the tapering of the pores, modifying the CCP power is a powerful tool to improve the pore geometry.

\section{vii) Distance between substrate and source}

Modifying the distance between the source and the substrate is expected to have a pronounced effect on the composition of the etching plasma as it arrives on the wafer. Upon increasing the distance, the ion density decreases, whereas the fluorine radical density remains more or less constant [181]. Figure 4.12(A) shows that reducing the ion density in the plasma decreases the average etching rate slightly. This was also shown by the experiments 


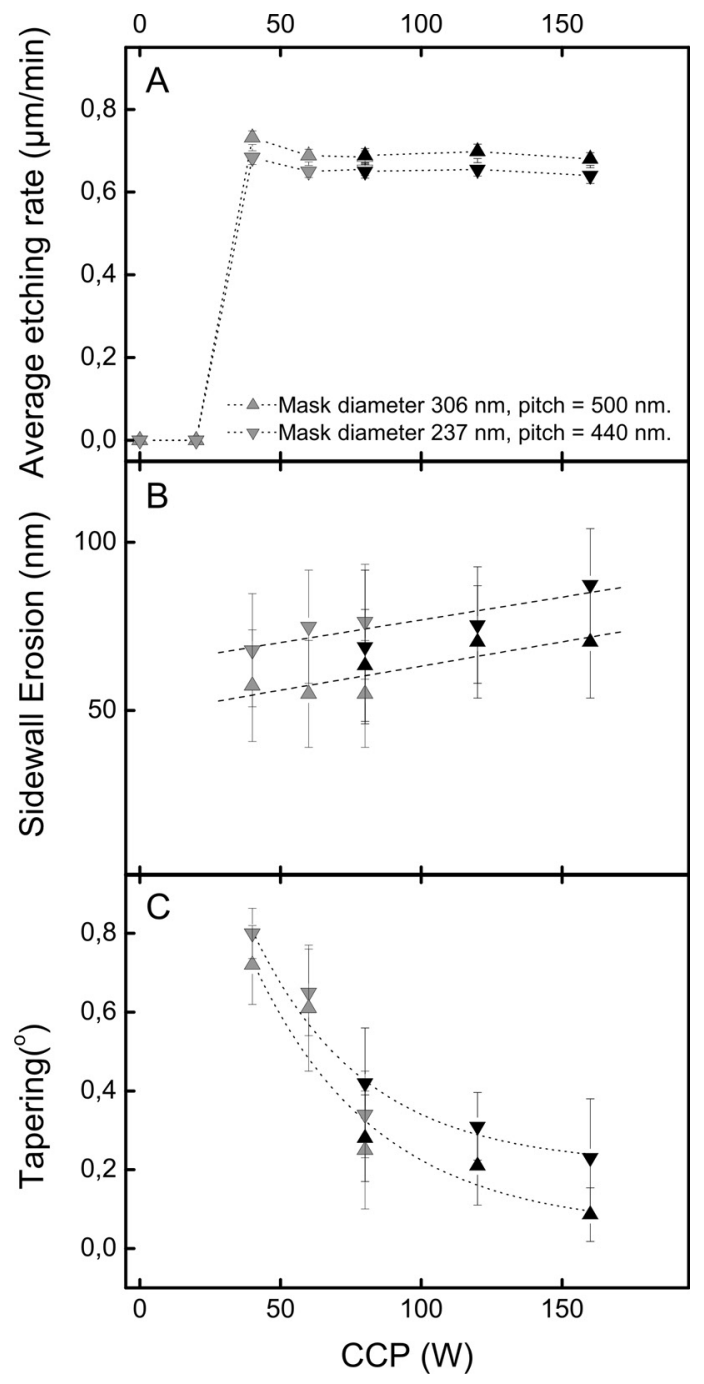

Figure 4.11: Average etching rate, sidewall erosion, and tapering of etched pores versus the CCP power varied during both the etching step and the deposition step. All dotted lines are guides to the eye. The dashed lines in B) are linear fits to the data. A) The average etching rate remains constant when varying the CCP power. At $20 W$ no etching occurs. B) The sidewall erosion iincreases with CCP power. This is due to a higher energy of the ions removing the polymer from the sidewalls during the etching step, and due to a less efficient deposition of polymer during the deposition step. C) At high CCP power the increased directionality of the plasma and the lower amount of sidewall protection reduces the tapering of the pores. 
where the ICP power was varied.

Upon increasing the substrate to source distance we observe a slightly increased sidewall erosion and a decreased tapering of the pores, see Figures 4.12(B) and (C). These results can only be explained by a decreased amount of sidewall protection, as less sidewall protection leads to more sidewall erosion and less tapering. Whereas the average etching rate is mainly influenced by what happens during the etching step, the effective sidewall protection is influenced by both the etching- and the deposition step. Since the composition of the plasma during both steps is influenced by the modified substrate to source distance, it is difficult to determine what contributes mostly to the change in sidewall protection. The available data only allows us to draw one general conclusion: modifying the substrate to source distance is of limited use to us as a tool to optimize our pores, since reducing the tapering leads to a lower average etching rate and more sidewall erosion and vice versa.

\subsubsection{Mask geometry}

The geometry of the mask through which the nanopores are etched influences both the etching rate and the sidewall erosion. In the next two sections we will discuss the effects of the mask aperture diameter and the pattern density.

\section{Mask aperture diameter}

As a consequence of the aspect ratio dependency of the etching process, we find deeper pores when the mask aperture diameter of the etched pores is larger for equal initial pattern densities, see Figure 4.7. In order to systematically quantify the dependence on mask aperture diameter, we plotted in Figure 4.13(A) the average etching rate versus the mask aperture diameter for experiments with different flows of $\mathrm{SF}_{6}$. The dashed lines are fits to the data that show a linear relation between the average etching rate and the diameter of the holes on the chromium mask. It is expected that the average etching rate will level off at higher mask aperture diameters, based on results described in literature [160, 163, 175, 184].

Recent cryogenic reactive ion etching experiments of large micrometer sized one-dimensional trenches in silicon show that the rate of sidewall erosion is equal for differently sized masks [185]. To test if this is also the case for our two-dimensional patterns and our etching process, we have plotted in Figure 4.13(B) the sidewall erosion versus the mask aperture diameter for the experiments with different flows of $\mathrm{SF}_{6}$. The Figure shows that the diameter of the mask aperture does not influence the amount of sidewall 


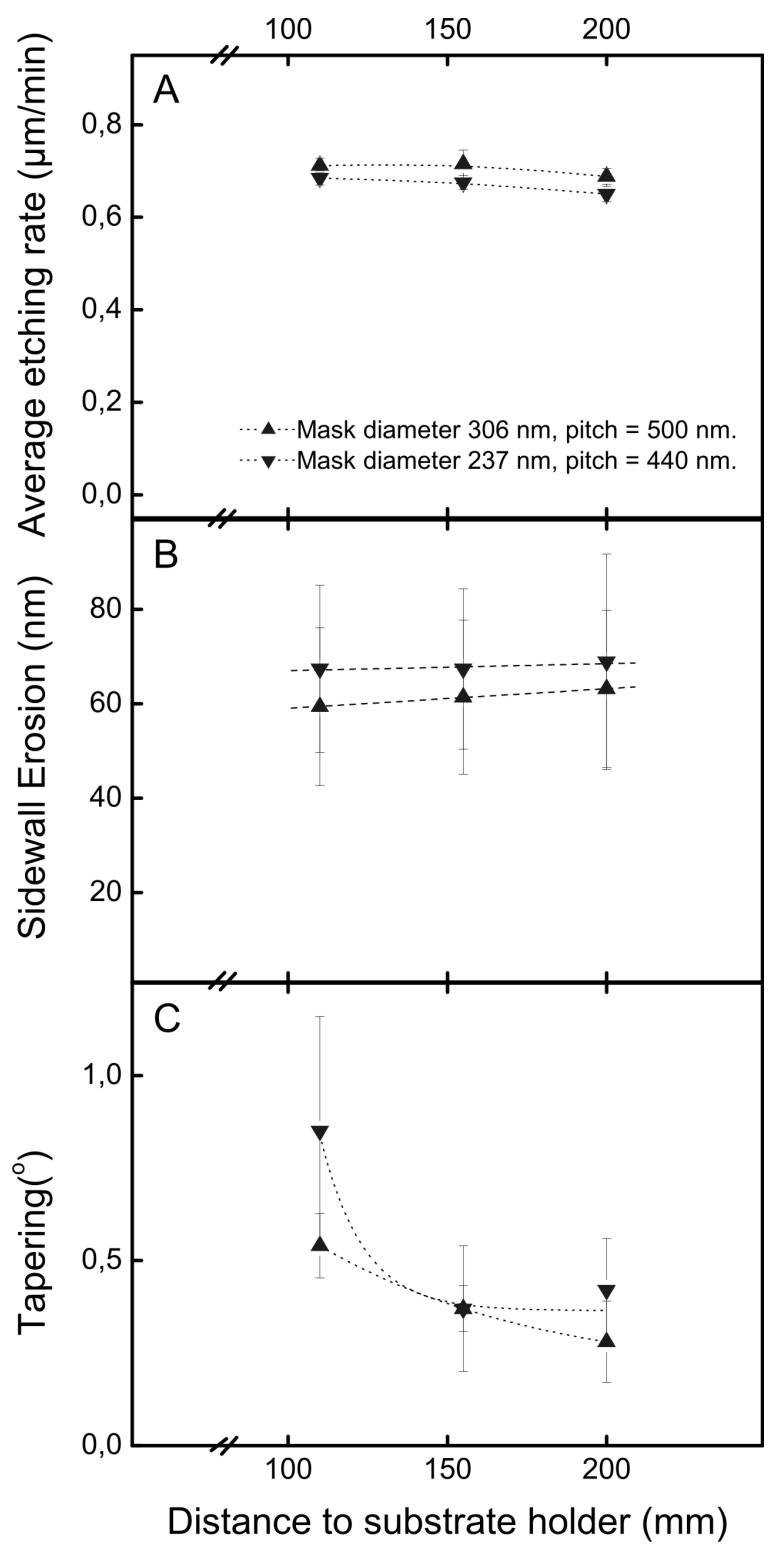

Figure 4.12: Average etching rate, sidewall erosion, and tapering of etched pores versus the distance between plasma source and substrate. All dotted lines are guides to the eye. The dashed lines in B) are linear fits to the data. A) Increasing the distance between plasma source and substrate leads to a lower average etching rate. This is due to a lower ion density. B) and C) Upon increasing the substrate to source distance we observe a slightly increased amount of sidewall erosion and a decreased tapering of the pores. This is explained by a decreased relative amount of sidewall protection. 


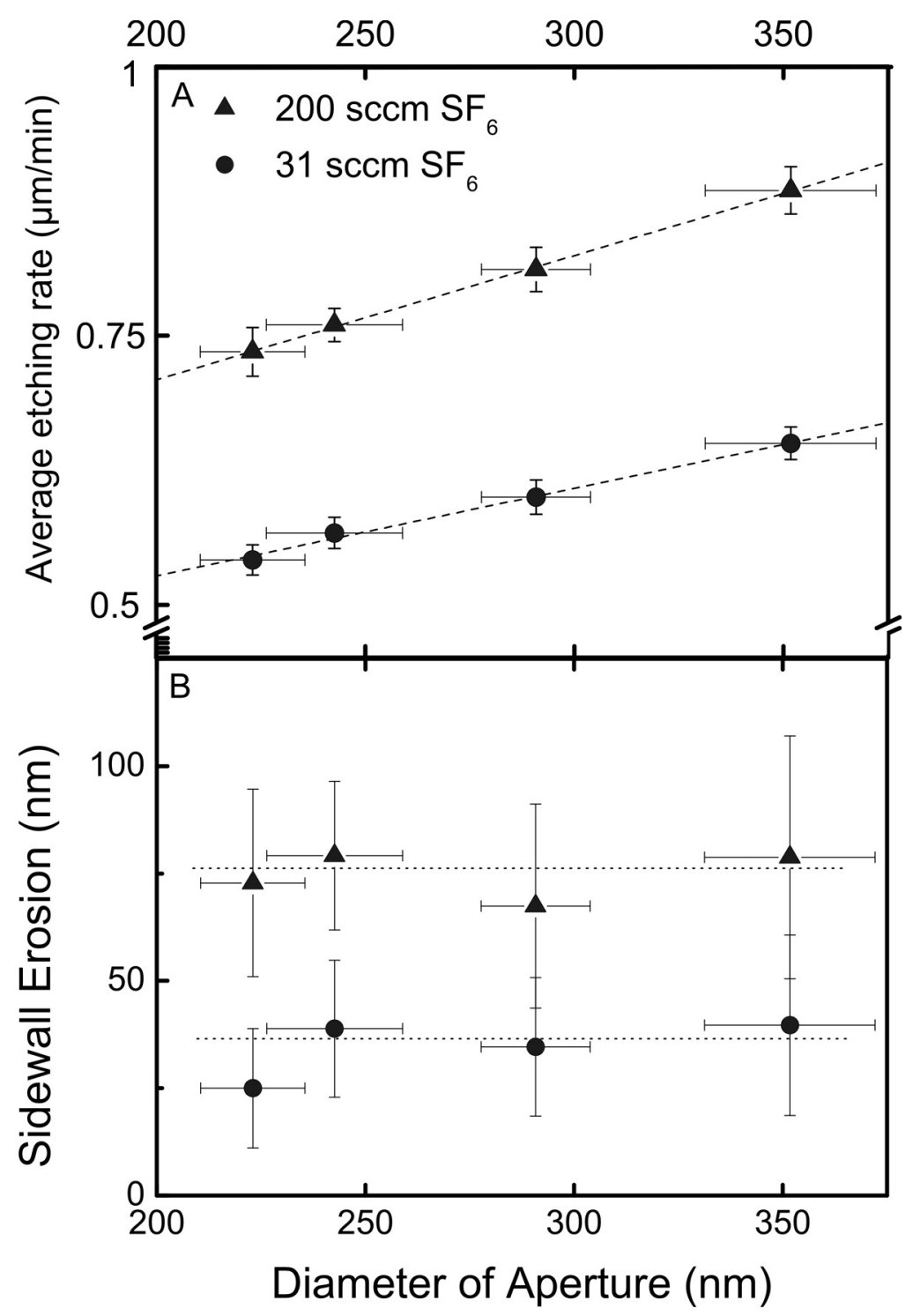

Figure 4.13: A) Average etching rate versus mask aperture diameter after 6 minutes etching with 31 and $200 \mathrm{sccm} S F_{6}$. The dashed lines are linear fits to the data. The confidence level of the fits was $95 \%$ and the coefficients of determination were found to be $>0.99$. B) Measured sidewall erosion versus mask aperture diameter for etching experiments with 31 and $200 \mathrm{sccm} S F_{6}$. The dotted lines serve as guides to the eye. 
erosion. Two of our data points were found to be exceptional, namely the holes with the lowest diameter at flows of 31 and of $62 \mathrm{sccm}$ (not shown in the Figure). Pores grown from these displayed even less sidewall erosion. For design purposes it is convenient if sidewall erosion is independent of mask aperture, as it allows a straightforward extrapolation from differently sized patterns to the expected diameter of desired pores.

\section{Influence of the pattern density on the sidewall erosion}

In the previous sections we observed that the rate of sidewall erosion is a) not aspect ratio dependent, and b) is not influenced by the diameter of the chromium mask. To see whether there is an influence of the pattern density on the rate of sidewall erosion, we plotted in Figure 4.14 the rate of sidewall erosion versus the initial pattern densities of the etched pores. The raw data for the sidewall erosion at $\phi=5.6$ and $11 \%$ are shown in Figure 4.6(B). The results in Figure 4.14 show that with increasing pattern density, the rate of sidewall erosion slightly decreases. We tentatively explain this result by considering two different possible mechanisms.

The first effect is the microloading effect $[176,186]$, which causes the amount of fluoride radicals to be relatively depleted at higher pattern densities compared to lower pattern densities. Therefore the sidewall erosion, caused by the fluoride radicals diffusing through the polymer protective layer, is reduced at high pattern densities compared to the sidewall erosion at lower pattern densities.

The second effect is the sidewall charging [187]: ions colliding with the sidewalls of the etched pores will leave their charge since the sidewalls are covered with an insulating polymer layer. This charge creates an electrostatic field which repels next ions, therefore reducing sidewall erosion. The charge on the sidewall will be compensated with electrons from the silicon behind the polymer layer. At higher initial pattern densities there is less bulk silicon, and therefore less electrons are available to move through the polymer layer. The ion induced charges will be less rapidly compensated and more ions will be repelled. Consequently the sidewall erosion will be less at higher pattern densities compared to lower pattern densities.

Since the initial pattern density is determined by design, it is not possible to use pattern density as a tool to reduce sidewall erosion. Fortunately, however, photonic crystals require structures with a high pattern density where only low rates of sidewall erosion occur. 


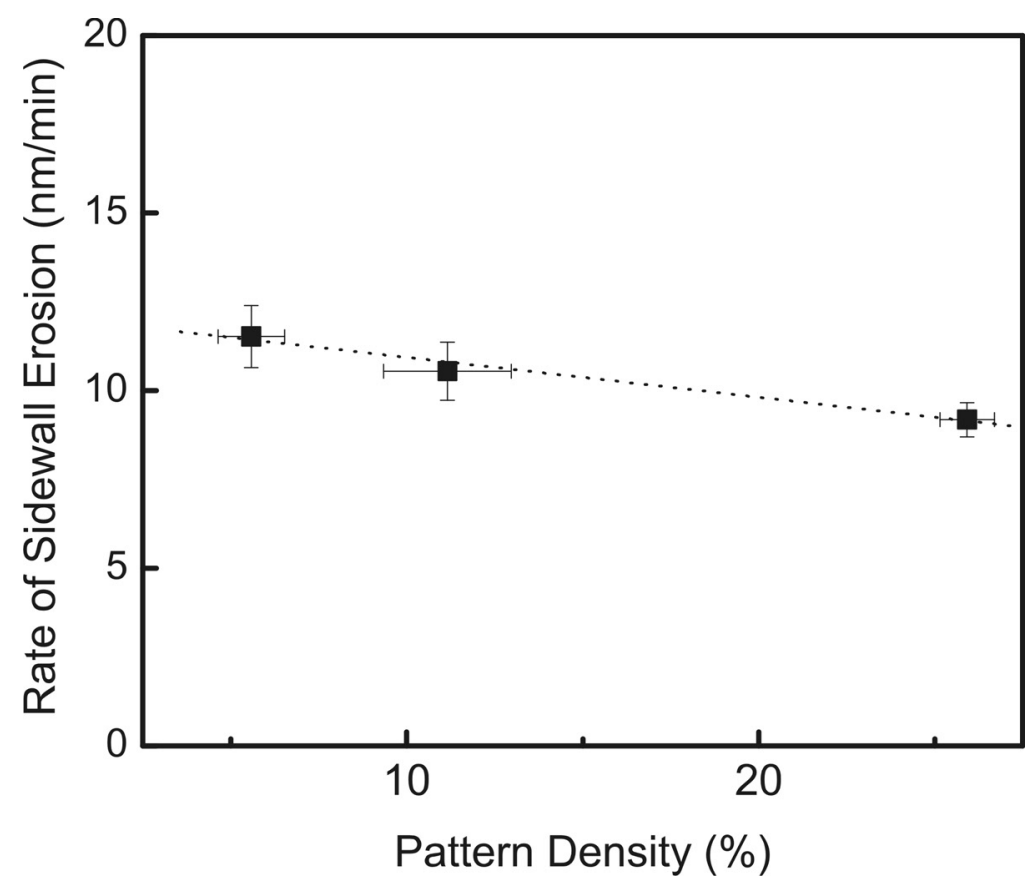

Figure 4.14: Rate of sidewall erosion as a function of initial pattern density. The dotted line serves as a guide to the eye. At higher pattern densities, the rate of sidewall erosion is reduced. In these experiments the flow of $S F_{6}$ was $125 \mathrm{sccm}$.

\subsection{Theoretical analysis of time dependent pore depth}

Ion angular distribution and image force In Bosch reactive ion etching the etching rates are usually aspect ratio dependent [163]. For submicron structures with high aspect ratios, two mechanisms have been determined to contribute most significantly. The first mechanism is referred to as the ion angular distribution [163, 188]: due to collisions of ions with gas and the thermal motion of the ions in the plasma glow, ions will travel with an angular distribution towards the pores. Due to this angular distribution of the ions, an increasing fraction of the ions will hit the sidewall of the pores, and less ions are available to etch the bottom of the pores when the pores get deeper. Consequently the average etching rate decreases as the aspect ratio increases. The ions that hit the sidewalls will cause erosion of the protective layer and therefore increase the amount of sidewall erosion. The second mechanism is commonly referred to as image force or image potential, see 
e.g. $[163,188,189]$ : a charged particle close to a solid material is attracted due to influencing fields. When a pore or trench is etched, such an image force attracts the incident ions towards the sidewalls and even when the ions are perfectly collimated, they will be deflected towards the sidewall. As the aspect ratio increases, more ions will hit the sidewall of the pores and less ions will be available to etch the bottom of the pores. The ions that hit the sidewalls will erode the protective layer and therefore increase the amount of sidewall erosion.

\section{Numerical integrations}

To interpret the results of our experiments, we compare our measured pore depths $h$ versus etching duration from Figure 4.6(A) with a theoretical model describing the reactive ion etching process. We apply a model from references $[188,190]$ for one-dimensional trenches to our two-dimensional pore arrays, since we expect the dynamics of the etching to be similar. In the model, the aspect ratio dependency of the etching rate of the process is described by considering the ion angular distribution and the image force effects separately, as well as considering both effects combined. Moreover we would like to investigate whether image force effects are more dominant in our sub-micrometer pores, in contrast to micrometer wide trenches where image force effects can be neglected until very high aspect ratios.

i) When only the image force effects are considered, the etching rate $R$ as a function of aspect ratio $A$ at etched time $t$ is described by

$$
\frac{R(t)}{R_{\max }}=1-\left(\frac{k \cdot A(t)^{2}}{E_{k i n} \cdot D(t)}\right)^{\left(\frac{1}{3}\right)}
$$

Here, $E_{k i n}$ is the kinetic energy of the ions that is equal to around $40 \mathrm{eV}, k$ is a constant equal to $1 \cdot 10^{-9} \mathrm{eVm}, D(t)$ is the measured diameter of the pores at time $t$, and $R_{\max }$ is the maximum etching rate.

ii) In the case where only the ion angular distribution is considered the etching rate $R(t)$ as a function of aspect ratio $A(t)$ is described by

$$
\frac{R(t)}{R_{\max }}=\frac{A_{c}}{A(t)}
$$

where $A_{c}$ is known as the critical aspect ratio [188]. This equation is valid for $A>A_{c}$. For $A<A_{c}, R(t)=R_{\max }$.

iii) The etching rate $R(t)$ as a function of aspect ratio $A(t)$ when considering both the ion angular distribution and the image force effects is described 
by multiplying equations (4.3) and (4.2):

$$
\frac{R(t)}{R_{\max }}=\frac{A_{c}}{A(t)} \cdot\left[1-\left(\frac{k \cdot A(t)^{2}}{E_{k i n} \cdot D(t)}\right)^{\left(\frac{1}{3}\right)}\right] .
$$

This equation is valid for $A>A_{c}$. When $A<A_{c}$ only the image force effects contribute and equation 4.2 applies.

We assume that all aspect ratios in Figure $4.6(\mathrm{C})$ are higher than $A_{c}$. To calculate the depth of the pores versus etching time, we have integrated equations (4.2), (4.3), and (4.4) numerically. To perform numerical integrations, the above equations had to be rewritten. Firstly $A(t)$ is rewritten as

$$
A(t)=\frac{h(t)}{D(t)}
$$

with $h(t)$ the calculated depth of the pores. $D(t)$ was determined by modeling the measured pore diameter versus etching time with a straight line (see Figure 4.6(B) ):

$$
D(t)=\alpha \cdot t+\beta .
$$

Fit parameter $\alpha$ represents two times the rate of sidewall erosion. Using equations (4.5) and (4.6), equation (4.2) was rewritten to

$$
R(t)=\frac{d h}{d t}=\left[1-\left(\frac{k \cdot h(t)^{2}}{E_{k i n} \cdot(\alpha \cdot t+\beta)^{3}}\right)^{\left(\frac{1}{3}\right)}\right] \cdot R_{\max } .
$$

Here $R_{\max }$ is taken to be an adjustable parameter. Equation (4.3) is rewritten to

$$
R(t)=\frac{d h}{d t}=\frac{(\alpha \cdot t+\beta) \cdot K}{h(t)},
$$

where $K=A_{c} \cdot R_{\max }$ is an adjustable parameter. Finally equation (4.4) is rewritten as

$$
R(t)=\frac{d h}{d t}=\left[1-\left(\frac{k \cdot h(t)^{2}}{E_{k i n} \cdot(\alpha \cdot t+\beta)^{3}}\right)^{\left(\frac{1}{3}\right)}\right] \cdot \frac{(\alpha \cdot t+\beta) \cdot K}{h(t)},
$$

again with $K$ as adjustable parameter. Numerical integrations were performed using the Runge-Kutta-Fehlberg 4th to 5th order procedure in Maple 9. We fitted the numerically integrated equations $4.7,4.8$, and 4.9 to the 
experimental data for $h(t)$ in Figure 4.6(A), using reduced $\chi^{2}$ minimization. In the procedure the depth of the pores at $t=3 \mathrm{~min}$ was fixed at their measured values.

i) When trying to fit with equation (4.7), where only the image force effects are considered, it was not possible to find a good match between fitted- and experimental data within reasonable conditions for $R_{\max }$. Therefore image force effects alone can be excluded as the sole mechanism responsible for the aspect ratio dependency of the etching rates.

ii) When fitting equation 4.8, where only the ion angular distribution is considered, the fits yielded values of $K=5.8 \mu \mathrm{m} / \mathrm{min}$ for the upward triangles and of $K=5.7 \mu \mathrm{m} / \mathrm{min}$ for the downward triangles. The goodness-of-fit $\chi^{2}$ values [191] were 11.9 and 6.69 respectively. These $\chi^{2}$ values are elevated, because the reported error bars do not reflect the intrinsic variabilities between separate etching experiments, which could not be quantified. From our experimental data we find that $R_{\max } \geq 0.85 \mu \mathrm{m} / \mathrm{min}$. This means that the obtained $K$ values correspond to critical aspect ratios $A_{c} \leq 6.8$ and 6.7 respectively, which is well below the measured aspect ratios at $t=3 \mathrm{~min}$, and validates our initial assumption that all reported aspect ratios in Figure 4.6(C) are higher than $A_{c}$.

iii) In the case where both the ion angular distribution and the image force are considered (equation 4.9), best fits were found for $K=7.3 \mu \mathrm{m} / \mathrm{min}$ for the upward triangles and for $K=7.5 \mu \mathrm{m} / \mathrm{min}$ for the downward triangles. Our fits yielded $\chi^{2}$ values of 12.4 and 6.61 respectively. These $K$ values correspond to critical aspect ratios $A_{c} \leq 8.5$ and 8.8 respectively, which is closer to the measured aspect ratios at $t=3 \mathrm{~min}$, but still equal to or below that value. Again, this result validates our initial assumption that all reported aspect ratios in Figure 4.6(C) are higher than, or at least equal to, $A_{c}$.

From the agreement between the fits of equations 4.8 and 4.9 , and our data, we conclude that for aspect ratios higher than the critical aspect ratio $A_{c}$ the ion angular distribution is the most pronounced mechanism that determines the aspect-ratio dependency of our etching rates. When considering both ion angular distribution and image force as the combined mechanisms, we also find reasonable fits, but no improvement. Therefore a contribution of image force effects on the observed etching rates is probably small and can be neglected.

Analytical theory for the ion angular distribution

In the situation that only the ion angular distribution affects the etching process, it is possible to derive an analytical expression for the depth of the 
pores as a function of etching time. This analytical expression is used to determine $R_{\max }$ and $A_{c}$ of our process. This expression for $h(t)$ is derived similar as in [188] and [190], with the modification that here the sidewall erosion is included:

$$
h(t)^{2}=\frac{h_{c}^{2}}{\left(\alpha t_{c}+\beta\right) t_{c}}\left(\alpha t^{2}+2 \beta t-\beta t_{c}\right),
$$

with $t_{c}$ the time, and $h_{c}$ the depth of the pores at which the critical aspect ratio $A_{c}$ is reached. Both $t_{c}$ and $h_{c}$ are free parameters. This equation is valid for $t \geq t_{c}$. For $t<t_{c}, h(t)=R_{\max } \cdot t$. When fitting equation 4.10 to our data we obtain solutions for $t_{c}$ and $h_{c}$. Given that $h_{c}=A_{c}\left(\alpha t_{c}+\beta\right)$ and that $h_{c}=R_{\max } \cdot t_{c}$, we can calculate $R_{\max }$ and $A_{c}$. For the downward triangles we find $R_{\max }=0.73 \pm 0.36 \mu \mathrm{m}$ and $A_{c}=7.9 \pm 2.1$, with a goodness-of-fit $\chi^{2}$ of 6.6. We get the following solution for the upward triangles: $R_{\max }=$ $0.85 \pm 0.21 \mu \mathrm{m}$ and $A_{c}=6.9 \pm 0.9$ with a $\chi^{2}$ of 12 . These results show that for an $\mathrm{SF}_{6}$ flow of $125 \mathrm{sccm}, A_{c}$ is below 8 and that $R_{\max }$ is around $0.85 \mu \mathrm{m}$, once again validating our initial assumptions. In Figure 4.6(A) we plotted the combined results of the fits of the measured data with equation 4.10 , and the results for the depth of the pores $h(t)$ at etching times below $t_{c}$, where $h(t)=R_{\max } \cdot t$. These plots show that our measured data is indeed well described by an ion angular dependence on the etching rate. In addition, these plots are very useful to determine what etching time is needed to acquire pores with a minimum depth requirement.

An advantageous feature of the analytical modeling is that we can derive an expression for the maximum aspect ratio $A_{\max }$. By dividing equation 4.10 by the expression $(\alpha \cdot t+\beta)^{2}$ and subsequently taking the limit of time $t$ to infinity, an expression for $A_{\max }^{2}$ is obtained:

$$
A_{\max }^{2}=\lim _{t \rightarrow \infty} A^{2}=A_{c} \cdot \frac{R_{\max }}{\alpha}
$$

While our number of data points is too small to obtain a well-constrained value for $A_{\max }(17 \pm 10$ and $16 \pm 14$, for the upward and downward triangles respectively), equation 4.11 gives insight in what mechanism should be improved to further increase the aspect ratio of our pores.

In this equation we see that increasing both $R_{\max }$ and $A_{c}$ have the beneficial effect of increasing the maximum obtainable aspect ratio $A_{\max }$. Reducing the rate of sidewall erosion (the rate of sidewall erosion equals $\alpha$ over 2) will also improve $A_{\max }$. Reducing the pressure in the system, or increasing the kinetic energy $E_{k i n}$ of the ions, will improve the ion angular distribution and therefore increase $A_{c}$. However, it is very difficult to obtain 
high $A_{c}$ values, as it requires a very high directionality of the ions in the plasma. It is possible to increase $R_{\max }$ by, e.g., increasing the flow of $\mathrm{SF}_{6}$. Unfortunately, while an increased flow of $\mathrm{SF}_{6}$ results in a higher etching rate $R_{\max }$, it also results in more sidewall erosion and these effects counteract in equation 4.11. The most promising option to increase the maximum aspect ratio $A_{\max }$ is to reduce the rate of sidewall erosion to values close to zero. For example, better sidewall protection can be achieved by enhancing the protecting step of the Bosch process (sections 4.3.3, and 4.5), increasing the maximum aspect ratio of our pores significantly.

\subsection{Ultimate etching}

Our experiments show that varying the power of the capacitively coupled plasma source, and varying the duration of the $\mathrm{C}_{4} \mathrm{~F}_{8}$ step have the most significant effect on the geometry of the obtained pores. In particular, varying the duration of the $\mathrm{C}_{4} \mathrm{~F}_{8}$ step allows us to minimize the sidewall erosion of the pores. To compensate for the increased amount of tapering, we increase the power of the capacitively coupled plasma source. In the next two sections we describe etching results for two different two-dimensional photonic crystal structures.

\subsubsection{Centered rectangular lattices}

We optimized the etching result of samples with a centered rectangular lattice like the one shown in Figure 4.2. All experiments were performed with $62 \mathrm{sccm}$ of $\mathrm{SF}_{6}$, a substrate holder distance of $200 \mathrm{~mm}$ and $1500 \mathrm{~W}$ ICP. The total etching times were chosen such that all experiments consisted of 180 cycles. One cycle contains one etching step and one deposition step. Table 4.3 shows the measured tapering, pore depth, diameter, and obtained aspect ratios for 6 separate experiments. The measurements shown are for pores with an initial mask aperture diameter of $222 \pm 13.4 \mathrm{~nm}$ and a pitch of 440 $\pm 8.8 \mathrm{~nm}$. Sets 3 and 4 are duplicates that show the good reproducibility of the process. The data in Table 4.3 confirm that increasing the flow of $\mathrm{C}_{4} \mathrm{~F}_{8}$ results in less sidewall erosion. At higher flows the pores get more tapered and we used the CCP power as a tool to limit this effect. Increasing the CCP power results in pores with less tapering. There is no significant effect on the tapering when increasing the CCP power from $160 \mathrm{~W}$ to $200 \mathrm{~W}$. Overall we see that increasing both the CCP power and the flow of $\mathrm{C}_{4} \mathrm{~F}_{8}$ results in pores with higher aspect ratios and sidewall tapering below $0.5^{\circ}$.

To summarize; the best settings for etching are: a flow of $62 \mathrm{sccm} \mathrm{SF}_{6}$ in 


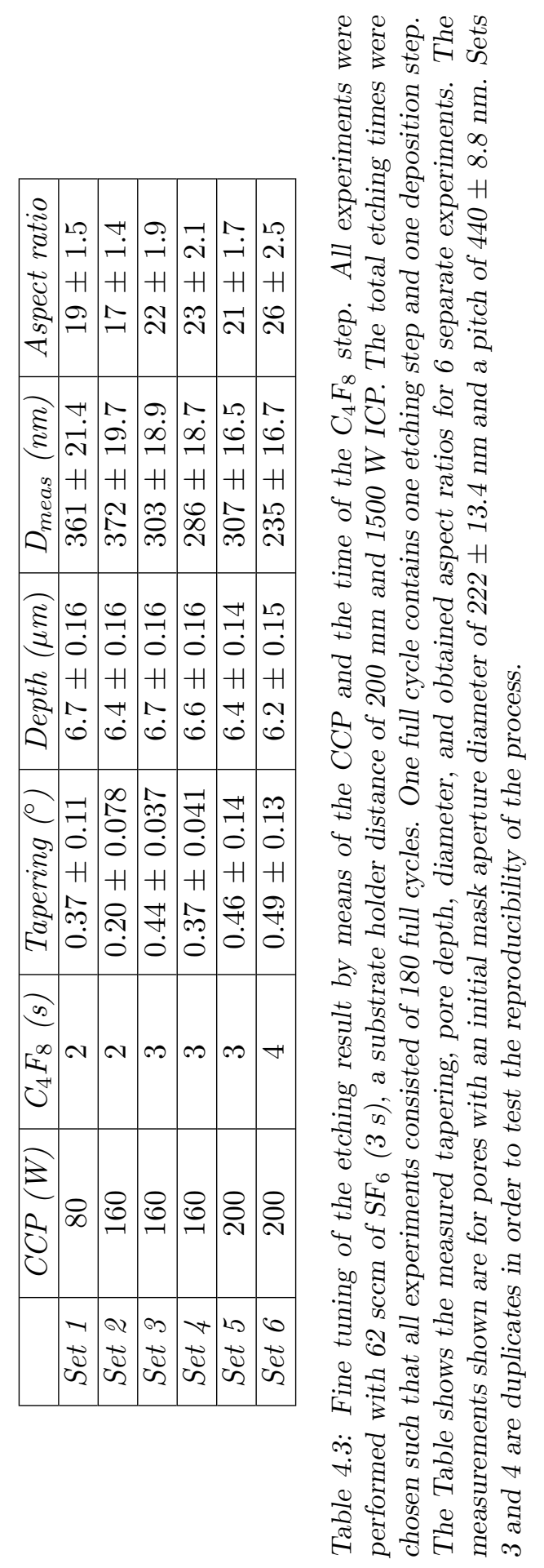




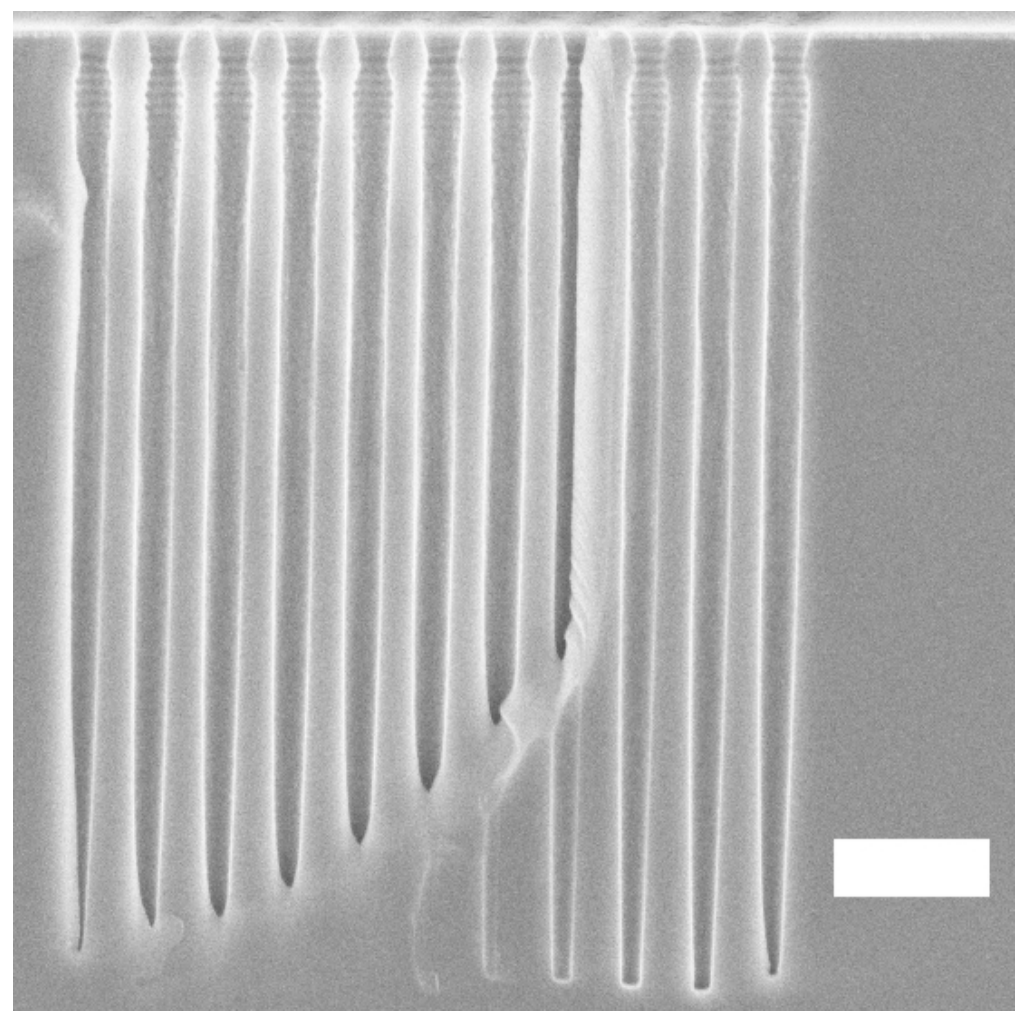

Figure 4.15: Pores etched with $200 \mathrm{~W} C \mathrm{CP}$ and 4 seconds of $\mathrm{C}_{4} \mathrm{~F}_{8}$. These pores have a diameter of $232 \pm 21 \mathrm{~nm}$ and a depth of $6.1 \pm 0.16 \mu \mathrm{m}$, which corresponds to a high aspect ratio of $26 \pm 2.5$. The tapering of these pores is $0.33 \pm 0.07^{\circ}$. With these settings the sidewall erosion of these pores was limited to $5 \pm 19 \mathrm{~nm}$. The scale bar represents $1 \mu \mathrm{m}$.

the etching step for a duration of 3 seconds, a flow of $200 \mathrm{sccm} \mathrm{C}_{4} \mathrm{~F}_{8}$ in the protecting step for a duration of 3 or 4 seconds, an ICP power of $1500 \mathrm{~W}$, CCP powers of 160 or $200 \mathrm{~W}$, and a substrate to source distance of $200 \mathrm{~mm}$. These settings yield pores with high aspect ratios, minimal sidewall erosion, and minimal tapering.

An example of pores from set 6 in Table 4.3 is shown in Figure 4.15. The pores are quite deep, exceeding $6 \mu \mathrm{m}$, with a high aspect ratio of $26 \pm 2.5$. This means that these pores are deep enough for optical experiments. The tapering of these pores has been reduced to $0.33^{\circ}$, and the sidewalls are smooth. To the best of our knowledge our arrays of nanopores have the highest aspect ratio reported in literature to date. 


\subsection{2 “ $\sqrt{2}$ " patterns}

In chapter 2 we introduced the three-dimensional inverse woodpile photonic crystals that we want to fabricate. Such crystals are obtained by etching two identical pore sets perpendicular to each other. These two pore sets have a centered rectangular lattice symmetry, similar to the crystals in the previous sections, but with different lattice parameters $a$ and $c$, see Figure 2.1. Here, the ratio of $a$ and $c$ equals $\sqrt{2}$, hence we refer to these patterns as " $\sqrt{2}$ " patterns. With optimized settings derived from the experiments in the previous sections, pores were etched with " $\sqrt{2}$ " patterned masks. For clarity, we will refer to the samples used in the previous section as "test" samples.

An example of a " $\sqrt{2}$ " pattern is shown in Figure 4.16(A). These structures were etched with a CCP power of $200 \mathrm{~W}$ in order to minimize sidewall tapering. The time of the $\mathrm{C}_{4} \mathrm{~F}_{8}$ step was chosen to be $3 \mathrm{~s}$ and $4 \mathrm{~s}$ (Figures 4.16(B) and (C), respectively). The resulting structures have dimensions and geometries comparable to the pores shown in the previous section. Again we find that increasing the time of the $\mathrm{C}_{4} \mathrm{~F}_{8}$ step leads to reduced sidewall erosion, as expected. From these experiments we tentatively conclude that the trends derived from the experiments on "test" samples are also valid for " $\sqrt{2}$ " patterns, even though the distribution of pores on the sample surface is different. The " $\sqrt{2}$ " patterns we used for our experiments were made by e-beam lithography. Therefore they are much less readily available, preparation of these samples is time consuming, and they are expensive. In the future we plan to make large " $\sqrt{2}$ " patterns by using deep UV lithography, but at any rate a methodology exists to optimize pore depths and pore geometry through experimenting on the "test" samples.

\subsection{Conclusions}

We have presented a "Bosch-type" reactive ion etching method to fabricate deep nanopores with high aspect-ratios of up to 26, in monocrystalline silicon. We etched pores with diameters smaller than $500 \mathrm{~nm}$ and pitches smaller than $1 \mu \mathrm{m}$, which have low tapering and smooth sidewalls. In order to etch these high-aspect ratio pores, the sidewall erosion was minimized, while maintaining a high etch rate. We have shown that the sidewall erosion is not influenced by the diameter of the holes in the mask. Furthermore, the rate of sidewall erosion is not aspect ratio dependent, and constant in time. Since the sidewall erosion decreases linearly with decreasing flows of $\mathrm{SF}_{6}$, a low $\mathrm{SF}_{6}$ flow must be balanced against the etching rate. We have concluded 

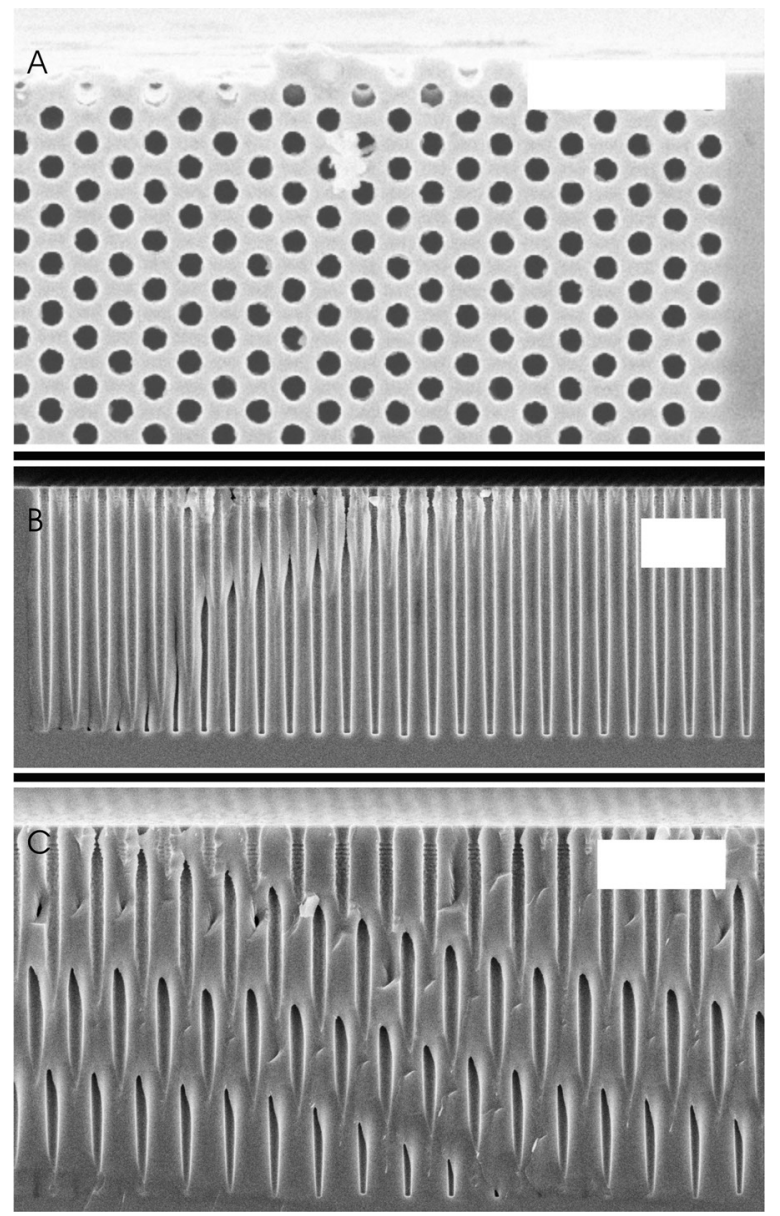

Figure 4.16: A) Top view of a " $\sqrt{2}$ " pattern etched with $200 \mathrm{~W} C C P$ and 3 seconds of $C_{4} F_{8}$. B) Cross sectional view of the same pores as A). These pores have a diameter of $306 \pm 24.5 \mathrm{~nm}$ and a depth of $5.9 \pm 0.15 \mu \mathrm{m}$, which corresponds to an aspect ratio of $19 \pm 2.0$. The tapering of these pores is $0.30 \pm 0.10^{\circ}$. C) Cross-sectional view of pores etched with $200 \mathrm{WCCP}$ and 4 seconds of $\mathrm{C}_{4} \mathrm{~F}_{8}$. The sidewall erosion of these pores is reduced compared to A) and B), resulting in a pore diameter of $241 \pm 16.6 \mathrm{~nm}$. The depth of these pores equals $5.8 \pm 0.13 \mu \mathrm{m}$. The aspect ratio is $24 \pm 2.2$. The increased time of the $C_{4} F_{8}$ step resulted in a higher tapering of $0.6 \pm 0.12^{\circ}$. The scale bars represent $2 \mu \mathrm{m}$. 
that etching with an $\mathrm{SF}_{6}$ flow near 62 sccm was optimal to achieve high aspect ratio pores. We have also shown that the sidewall erosion decreases with increasing pattern density, which is favorable for photonic crystal applications. Masks with smaller holes yield pores with higher aspect ratios, similar to results in literature on large one-dimensional trenches.

By evaluating experiments with different flows of $\mathrm{SF}_{6}$ and with different pattern densities we have determined that our etching process is aspect ratio dependent. Firstly, our time-dependence study shows that the etching rate decreases when the time etched, and thus the aspect ratio, increases. Secondly, our experimental data are well described by a theoretical model for aspect-ratio dependent reactive ion etching. At aspect ratios higher than $A_{c}$ the ion angular distribution appears to contribute most significantly to the aspect ratio dependency of the etching process. A contribution of image force effects is small and can be neglected. We calculated the critical aspect ratio $A_{c}$ and the initial etching rate $R_{\max }$ of our process with a flow of $125 \mathrm{sccm}$ $\mathrm{SF}_{6}$. Furthermore, we show that reducing the amount of sidewall erosion is the most powerful tool to improve the maximum obtainable depth of the pores. Less sidewall erosion is realized by applying more sidewall protection by increasing the time of the protection step of the Bosch process.

The influence of a number of parameters on the average etching rate, sidewall erosion and pore tapering was studied. The parameters were the flow of $\mathrm{C}_{4} \mathrm{~F}_{8}$, the duration of the $\mathrm{C}_{4} \mathrm{~F}_{8}$ step, the ICP power of the etching step, the CCP power, and the source to substrate distance. Besides changing the flow of $\mathrm{SF}_{6}$, modifying the duration of the $\mathrm{C}_{4} \mathrm{~F}_{8}$ step and the CCP power were shown to be the best tools for improving the depth and geometry of the pores. By fine tuning the CCP power and the time of the $\mathrm{C}_{4} \mathrm{~F}_{8}$ step we have managed to obtain deep pores, with high aspect ratios, smooth sidewalls, and a low amount of tapering. We have seen that trends derived from etching experiments on the test samples are also valid for our " $\sqrt{2}$ " patterns.

Our modified Bosch reactive ion etching process is very suitable for the etching of arrays of deep nanopores. To the best of our knowledge the pores have the highest aspect ratio reported to date for arrays of nanopores in silicon, made with a reactive ion etching process. This enables the fabrication of photonic crystals with large volumes, which also opens an avenue towards the fabrication of three-dimensional crystal structures. Our fabrication process is CMOS compatible, which means that these structures can easily be integrated in existing silicon electronics manufacturing. 


\subsection{Appendix A}

For a two-dimensional array of pores, the pattern density $\phi$ is defined as

$$
\phi=\frac{S_{\text {hole }}}{S_{\text {unitcell }}} \cdot 100 \%,
$$

with $S_{\text {hole }}$ the surface of one hole in the mask, $S_{\text {unitcell }}$ the surface of the primitive unit cell of the lattice. Using a centered rectangular lattice, equation 4.12 can be rewritten to contain a linear relation between the square of the diameter and of the pitch:

$$
\phi=\frac{\frac{1}{4} \cdot \pi \cdot D^{2}}{a^{2} \cdot \sin (\varphi)} \cdot 100 \%,
$$

with $\varphi$ the lattice angle, in our case $\varphi=84.5^{\circ}$.

Therefore, the relation between the diameter $D$ and the pitch of the holes $a$ for a constant pattern density is linear

$$
D=b \cdot a,
$$

with $b$ the slope of the line.

Equations 4.14 and 4.13 combined yield an expression for the pattern density in terms of the experimentally determined slope:

$$
\phi=\frac{\frac{1}{4} \cdot \pi \cdot b^{2}}{\sin (\varphi)} \cdot 100 \% .
$$




\section{Chapter 5}

\section{Fabrication and testing of the holder wafer}

\subsection{Introduction}

To obtain three-dimensional inverse woodpile photonic crystals, two separate steps are required in which pores are fabricated, see chapter 2. Figure 5.1(A) shows an artist impression of a sample in which a first set of nanopores has been etched. The second set of pores needs to be etched perpendicular to the first, in the Figure indicated by tilting the sample by $90^{\circ}$. Properly aligning the position and direction of the two sets of pores with respect to each other is a necessity and is quite challenging. In recent literature a number of possible solutions to this particular challenge are reported.

In reference [67] three-dimensional woodpile crystals were fabricated by a double-angled etching technique. By two consecutive cryogenic reactive ion etch steps of $45^{\circ}$ with respect to the wafer surface, at an angle of $90^{\circ}$ between the two etch directions, a thin photonic crystal was obtained. Unfortunately, reference [67] lacks a description of the experimental methods. If, in a Gedankenexperiment, we try to adapt this work, we encounter a number of challenges and questions that disencourage us to adapt the double-angled reactive ion etch technique. Examples of the challenges include the required extensive modification of the etching equipment, compromised homogeneity of the etching result, and non-trivial patterning of the second pore sets.

In references [192], [69], and [193] focused ion beam milling was used to etch $2^{\text {nd }}$ and $3^{\text {rd }}$ sets of pores in existing macroporous structures. They were able to fabricate, respectively, three-dimensional "Yablonovite"-like and inverse woodpile photonic crystals. In this fashion existing two-dimensional structures can be quickly turned into three-dimensional photonic crystals. Regrettably, focused ion beam milling only allows the milling of relatively small areas in the order of $15 \times 15 \mu \mathrm{m}^{2}$. This limits the volume of the 
obtained photonic crystals severely (unless a disproportional amount of time is invested). Furthermore, although processing of larger areas can be done by subsequent milling of patterns next to each other (so-called "stitching"), this typically leads to alignment errors between the separate patterns. In addition, in our experience, pores milled with a focused ion beam tend to display significant tapering which is undesirable. Nonetheless, we recognize this method as a powerful way to fabricate "prototype" three-dimensional photonic crystals and we also show similar results in chapter 7 . However, since ultimately we aim to obtain crystals with large volumes, an alternative route was developed.

In order to etch a second set of pores in a controlled fashion, we investigated the possibility to fix an existing sample in place using a separately fabricated holder wafer. This holder wafer was already introduced in chapter 2, section 2.3.3. Figure 5.1(A) shows an impression of sample in which a first set of nanopores was etched. We refer to these samples as "2D-crystal samples". Prior to placing the 2D-crystal sample in the holder wafer, the surface that will be etched needs to be polished to enable deposition of mask material and resist, see for details section 5.3. In order to etch the second set of pores, the polished 2D-crystal sample is tilted by $90^{\circ}$ and placed in a slot in the holder with high precision to maintain the perpendicular orientation of the etched pores to the second etch direction. Figure 5.1(B) shows a schematic topview of the holder wafer. Slots which hold the 2D-crystal samples in the holder wafer are shown in white. Their dimensions are chosen such that the 2D-crystal samples fit tightly. In this way, the 2D-crystal samples will not be able to fit in the slots in a rotated or tilted fashion, thus ensuring good alignment. Figure 5.1(C) shows the total wafer stack after glueing the samples in the holder wafer. The $2 \mathrm{D}$-crystal sample is visible in the middle of the construction as the grey square. It is held firmly in place by the silicon holder wafer. The use of a dummy wafer ensures perfect alignment of the surface of the 2D-crystal samples with the surface of the holder wafe, thus facilitating the deposition of mask material and spin coating of resist. Placing a "glue" wafer containing a thick layer of photoresist on top of the holder wafer with the 2D-crystal samples ensures a solid connection between sample and holder wafer such that the construction can withstand the mechanical stress induced by further processing such as spin coating. After hardening of the photoresist, the holder wafer and dummy wafer are separated and lithography and etching of the second set of pores in the 2D-crystal samples can proceed using standard processes and unmodified equipment. Here we will demonstrate the fabrication of a pattern in a chromium mask on top of a dummy sample by means of focused ion beam 

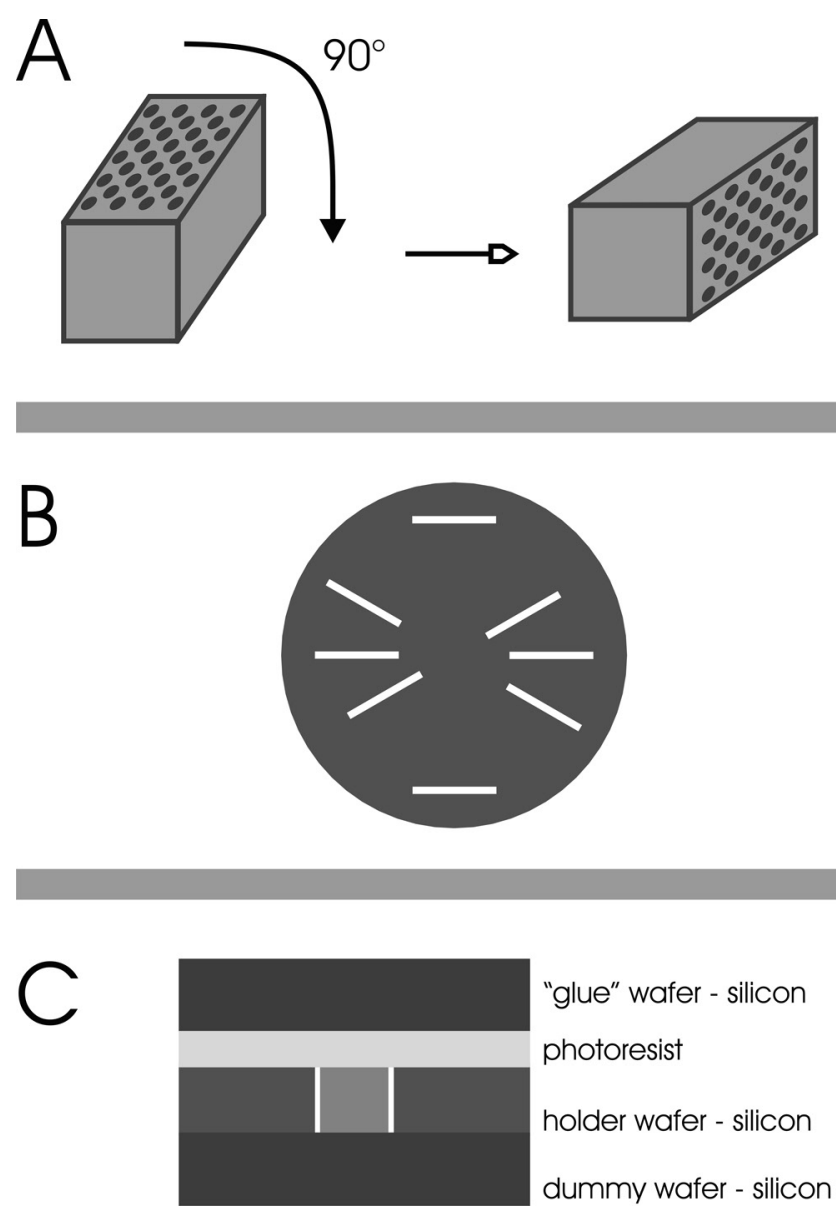

Figure 5.1: A) Artist impression of a 2D-crystal sample in which a first set of nanopores has been etched. The sample is tilted by $90^{\circ}$ and placed in a slot in the holder wafer to etch the second set of pores. The surface to be etched is polished prior to mounting the sample in the holder wafer. B) Schematic topview of the holder wafer. Slots in the holder wafer are shown in white. These hold the samples to be etched, ensuring good alignment. C) Schematic of the total wafer stack after glueing the samples in the holder wafer. The grey square in the middle is the sample. The sample- and holder wafer surfaces are aligned to each other by the dummy wafer. The are fixed in the holder wafer by the "glue" wafer and a layer of hardened photoresist. Next, the dummy wafer is removed and the surface of the $2 D$-crystal sample is exposed for further processing. 


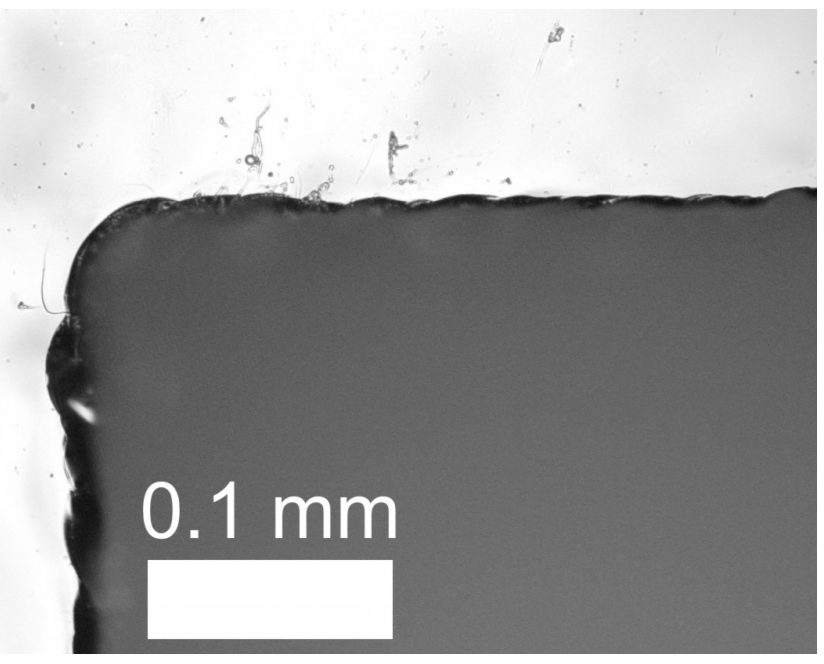

Figure 5.2: Optical micrograph of the corner of a large square slot cut out of a silicon wafer using a laser cutting machine. Although the walls of the hole are irregular, they are perpendicular to the wafer surface, which allows a tight fit of $2 D$-crystal samples. On the surface of the wafer, small sputtered silicon particles are visible as small dark spots.

milling, and test the alignment.

\subsection{Fabrication of the holder wafer}

For the holder wafer we used standard p-type $<100>$ oriented double side polished silicon wafers with a thickness of $525 \mu \mathrm{m}$ and a diameter of $100 \mathrm{~mm}$. Slots were cut in the wafers using a Rofin-Sinar 150 P2/CW YAG laser cutting machine, see Table 5.1. These slots hold the 2D-crystal samples in which a second set of pores will be etched. The slots are oriented radially to enable spin coating of photoresist. After cutting the slots, the wafers were first cleaned in an ultrasonic water bath for at least 5 min to remove loose sputtered silicon particles that were yielded by the laser cutting process. Thereafter, the holder wafers were immersed in a $25 \%$ (wt) $\mathrm{KOH}$ solution at $74^{\circ} \mathrm{C}$ for 30 to 60 minutes to etch remaining silicon particles. The wafers were rinsed with demineralized water afterwards.

Figure 5.2 shows a corner of a large square slot cut out of a silicon wafer using a laser cutting machine ${ }^{1}$. Importantly, the walls are perpendicular to

\footnotetext{
${ }^{1}$ Micrographs that are presented in this chapter are imaged by a Nikon Eclipse ME600L
} 
Table 5.1: Overview of settings used during laser cutting of the slots in the holder wafers. The slots were cut with a Rofin-Sinar 150 P2/CW YAG laser cutting machine.

\begin{tabular}{|l|c|}
\hline Setting & Value \\
\hline Current to the laser & $100 \mathrm{~A}$ \\
\hline Laser pulse length & $0.3 \mathrm{~ms}$ \\
\hline Laser frequency & $400 \mathrm{~Hz}$ \\
\hline Spotsize & $0.04 \mathrm{~mm}$ \\
\hline Diaphragm & $1.5 \mathrm{~mm}$ \\
\hline Cutting speed & $200 \mathrm{~mm} / \mathrm{min}$ \\
\hline Flow of of $\mathrm{O}_{2}$ delivered to the cutting area & $5 \mathrm{bar}$ \\
\hline
\end{tabular}
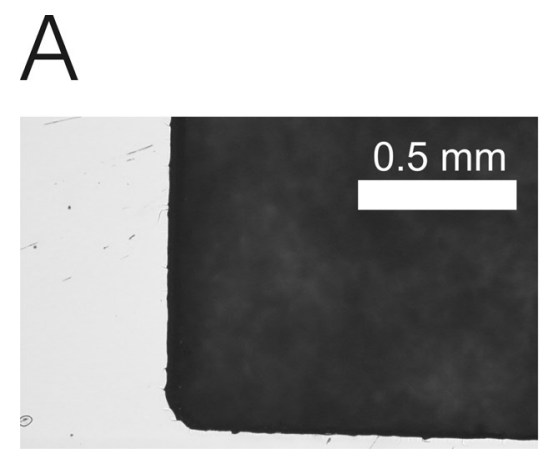

B

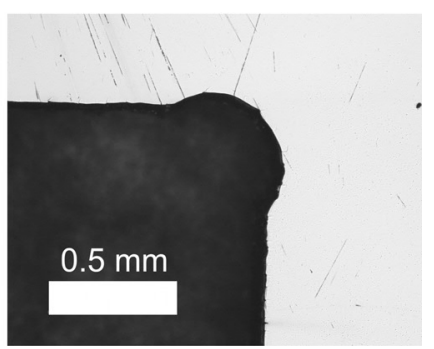

Figure 5.3: A) Optical micrograph of a slot cut in a silicon wafer. The programmed $90^{\circ}$ corner was rounded on the inside, see bottom left of the slot. Tightly placing a sample inside the slot is impossible because there is still silicon remaining where the sample should go. B) Optical micrograph of an improved slot cut in a silicon wafer. In this case the laser cutter was programmed to round the corner of the square on the outside. In this fashion, enough room is created for a sample to fit into the slot tightly with good alignment accuracy.

the wafer surface. This allows a tight fit of samples. The walls of the square slot appear irregular in the sense that the hole walls are not straight. The hole wall appears to be rather similar to a very blunt saw edge, but fortunately this does not influence the perpendicular orientation of the sidewall.

When the laser cutter is programmed to fabricate a $90^{\circ}$ corner, it does

optical microscope, equipped with a DXM-1200F digital camera or by a LEO 1550 high-resolution scanning electron microscope. 
not produce a sharp corner due to the circular shape of the laser beam, see Figure 5.3(A). By programming the laser cutter to round the corner of the square on the outside, there is enough room created for a sample to go into the slot, see Figure 5.3(B). In this fashion, we are able to place samples inside the slots and maintain a tight fit and thus good alignment accuracy.

In conclusion, laser cutting proved to be an efficient way to prepare the holder wafers. This technology is readily available and provides good results. So-far, we have obtained $>10$ holder wafers, showing that the process is reproducible.

\subsection{Preparation of the cross-section surface of an etched sample}

2D-crystal samples small enough to fit in the holder wafer are retrieved from larger pieces of wafer in which the first set of pores were etched. Typically, these smaller pieces of sample are obtained by cleaving or dicing, which unfortunately results in significant damage to the cross-section, see Figure 5.4. Of these two options, dicing the samples typically results in the most significant damage. Figure 5.4(A) shows that many loose silicon pieces are produced, indicative of the amount of damage done. In addition, the Figure shows that many layers of etched pores are visible because a large crater is cut out by the dicing saw while ideally one would hope to see only one layer of pores. In Figure 5.4(B) is shown that when an etched sample is cleaved, this results in a rough-textured cross-section surface. Multiple layers of the etched pores can be seen, because the cleave is not exactly perpendicular to the wafer surface. Furthermore a piece of silicon seems to have broken of the upper right part of the structure. After etching of pores and fracturing the wafer, the exposed pores are to be patterned to provide a mask for etching of the second set of pores, see chapter 2. Unfortunately, surfaces as shown in Figures 5.4(A) and (B) are not flat enough to continue processing using resists and masks: alignment of the tilted samples in a holder wafer is significantly hampered by the rough texture of the cross-section surfaces and it is not possible to spin coat resist on such rough surfaces. To improve the flatness of the cross-section surface of the 2D-crystal samples, "tripod polishing" or "wedge polishing" was investigated.

Before polishing, any chromium mask material present on the 2D-crystal samples was removed by immersion in standard $\mathrm{MESA}^{+}$cleanroom chromium etchant. Initial polishing experiments on samples with nanopores, showed that the porous structure was too brittle to withstand the procedure. In 

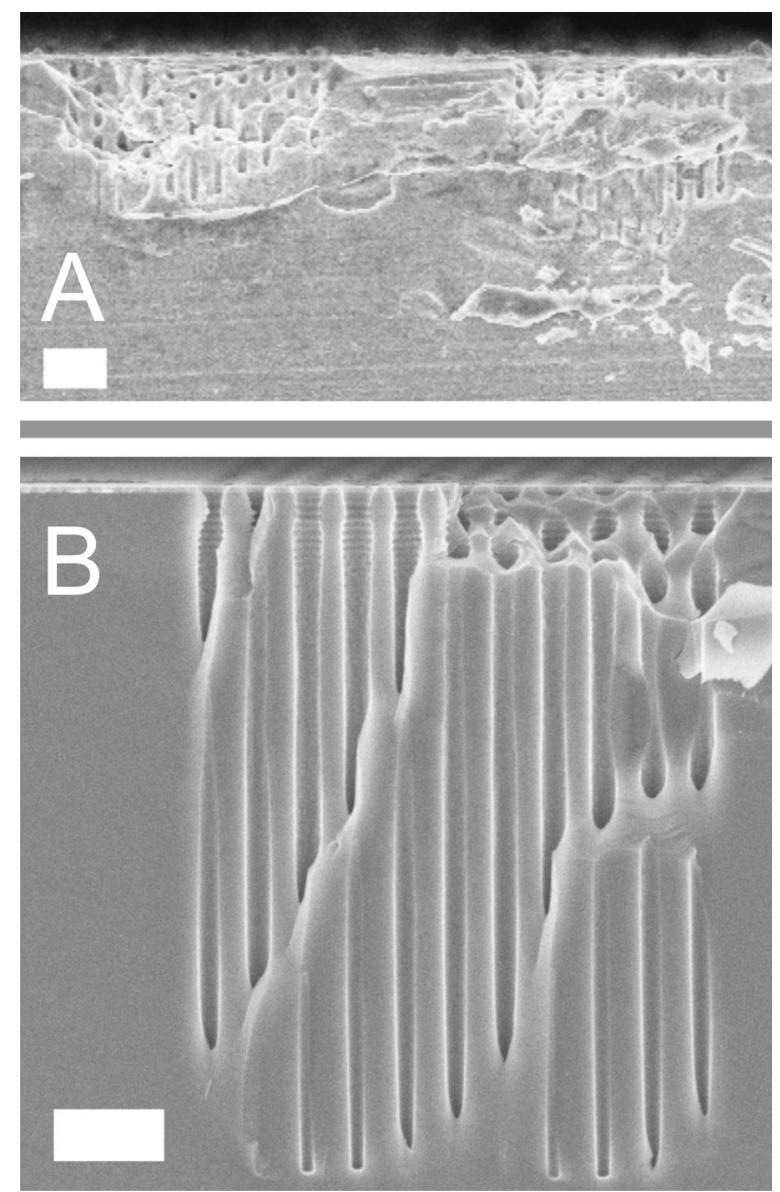

Figure 5.4: A) Scanning electron micrograph of a cross-section of an etched sample diced by a dicing saw. The scale bar equals $2 \mu \mathrm{m}$. Many loose pieces of silicon can be seen, as well as large craters that have been cut out by the saw. Because of these craters, many layers of etched pores are visible. The damage to this cross-section surface is significant. B) Scanning electron micrograph of a cross-section of a cleaved sample. The scale bar equals $1 \mu \mathrm{m}$. The sample is not flat, and multiple layers of etched pores are visible. The cleave is not perpendicular to the wafer surface. A piece of silicon has broken off the upper right section of the structure. Both surfaces are not suitable for etching of the second set of pores. 
Chapter 5. Fabrication and testing of the holder wafer
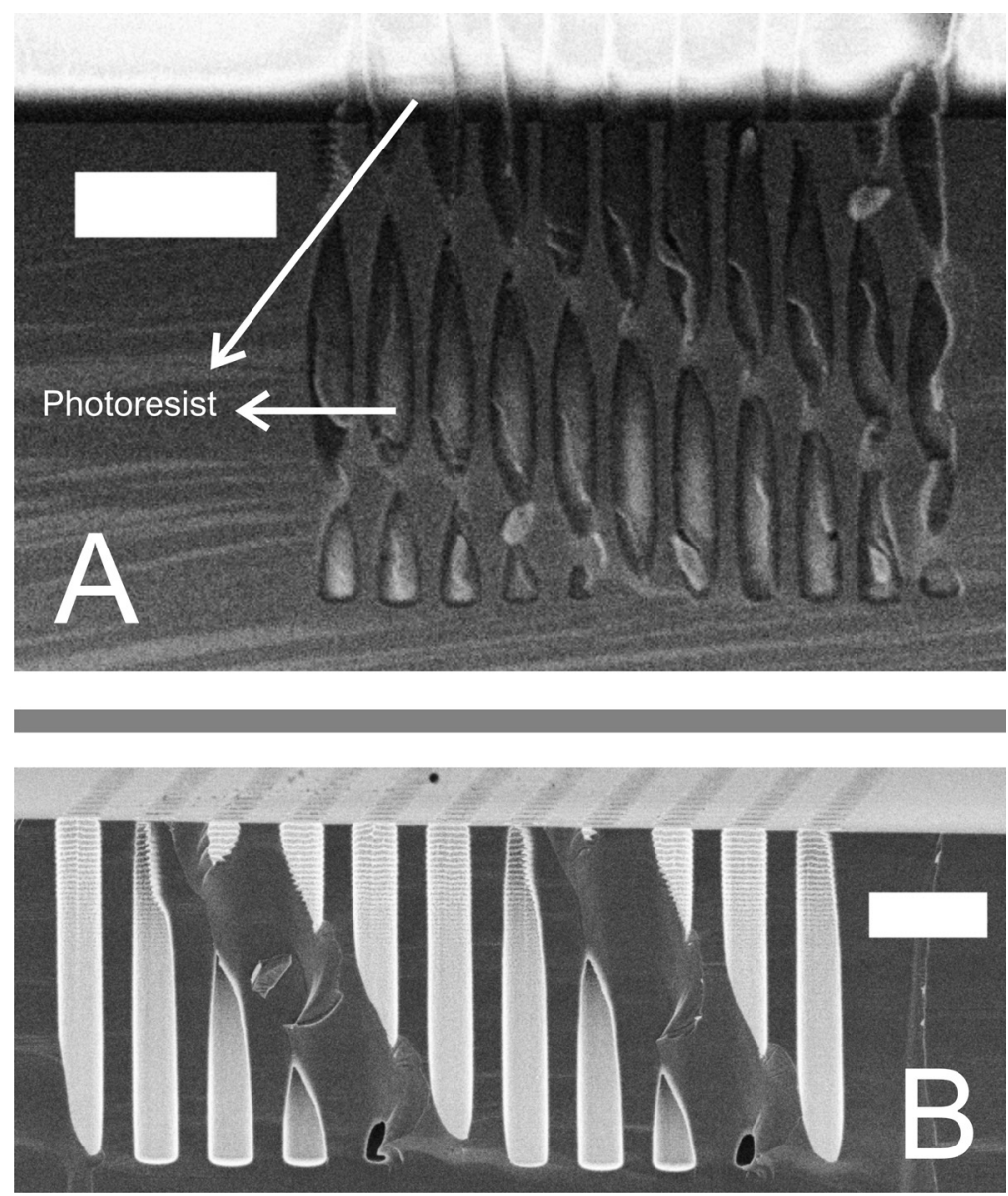

Figure 5.5: A) Scanning electron micrograph of a cross-section of etched pores in silicon filled with photoresist. The photoresist is visible on top of the sample and also inside the pores. The entire volume of the pores appears to be filled with photoresist. B) Scanning electron micrograph of a cross-section of pores in silicon after removal of the photoresist by calcination. The scalloping on the sidewalls of the etched pores is visible, suggesting that there is no photoresist left on the sidewalls on the pores. Also the surface of the etched wafer seems to be clean. For unknown reasons the sidewalls of the pores seem to be charging up. The scale bars equal $2 \mu \mathrm{m}$. 
order to improve the stability of the structure, a thick layer of photoresist $\left(\mathrm{MESA}^{+}\right.$, Olin 907/12) was deposited on the surface. Due to capillary forces, the photoresist also filled the etched pores, see Figure 5.5(A). The Figure also shows that the pores are almost completely filled with photoresist. The resist was hardened by heating to $120{ }^{\circ} \mathrm{C}$ for 10 seconds in order to render the material tough and resistant to mechanical strain and stress. An added beneficial effect of the filling is that it protects the inside of the pores from small pieces of polishing debris, which are very hard to remove. In case of the experiments shown in Figure 5.6, other photoresist $\left(\mathrm{MESA}^{+}\right.$, Olin $908 / 35)$ was used, which was hardened at $95{ }^{\circ} \mathrm{C}$ overnight. In this case the photoresist layer on top of the sample is even smoother and better defined.

The sample was mounted in a tripod polisher (South Bay Technology) [194] and carefully polished ${ }^{2}$ using, consecutively, $30 \mu \mathrm{m}, 15 \mu \mathrm{m}, 9 \mu \mathrm{m}, 6 \mu \mathrm{m}$, and $3 \mu \mathrm{m}$ diamond lapping films. The polish direction was from photoresist to silicon. Subsequently $1 \mu \mathrm{m}, 0.5 \mu \mathrm{m}$, and $0.1 \mu \mathrm{m}$ diamond lapping films were used during polishing in the reverse direction with the silicon wafer as front. Initially the lapping film was rotating at $30 \mathrm{RPM}$, but as the lapping film was changed to smaller particles sizes, this speed was reduced to 10 RPM. When polishing with the final lapping films of 0.5 and $0.1 \mu \mathrm{m}$, the film was not rotating, but the tripod polisher was carefully and without pressure moved over the surface of the film. In all cases the direction of movement was parallel to the etched pores and never perpendicular. With an optical microscope the polishing result was intermittently observed and when the result was found to be satisfactory, polishing was ceased. After polishing, the $2 \mathrm{D}$-crystal sample was rinsed.

To remove photoresist from the samples, the sample was heated to a temperature of $650{ }^{\circ} \mathrm{C}$ following the procedure outline in Table 5.2. At this temperature, the photoresist is calcinated, i.e., it is gasified and burned. After calcination the 2D-crystal sample was rinsed. Figure 5.5(B) shows that all photoresist was removed from the etched pores and the surface of the wafer. The scalloping on the sidewalls of the etched pores, caused by the Bosch reactive ion etch process, see chapter 4 , is still visible, confirming that there is no photoresist left on the sidewalls of the pores. It is not clear why the sidewalls of the pores are apparently charging up, as indicated by the bright white outlining of the pores.

Figure 5.6 shows a polished cross-section surface of an etched sample. The photoresist was removed by calcination after which the sample was rinsed. The cross-section surface is flat, without any craters damaging the etched

${ }^{2}$ Polishing experiments by E.G. Keim, central materials analysis laboratory, MESA ${ }^{+}$ Institute for Nanotechnology, Enschede, The Netherlands. 
Table 5.2: Overview of the calcination procedure used to remove photoresist from the samples.

\begin{tabular}{|c|r|}
\hline Step & Description \\
\hline 1 & From room temperature to $80{ }^{\circ} \mathrm{C}$ at $20{ }^{\circ} \mathrm{C} / \mathrm{h}$ \\
\hline 2 & Hold $80{ }^{\circ} \mathrm{C}$ for 60 minutes \\
\hline 3 & Heat from $80{ }^{\circ} \mathrm{C}$ to $650{ }^{\circ} \mathrm{C}$ at $120{ }^{\circ} \mathrm{C} / \mathrm{h}$ \\
\hline 4 & Hold $650{ }^{\circ} \mathrm{C}$ for 360 minutes \\
\hline 5 & Cool down to $25{ }^{\circ} \mathrm{C}$ in 6 hours \\
\hline 6 & End program \\
\hline
\end{tabular}

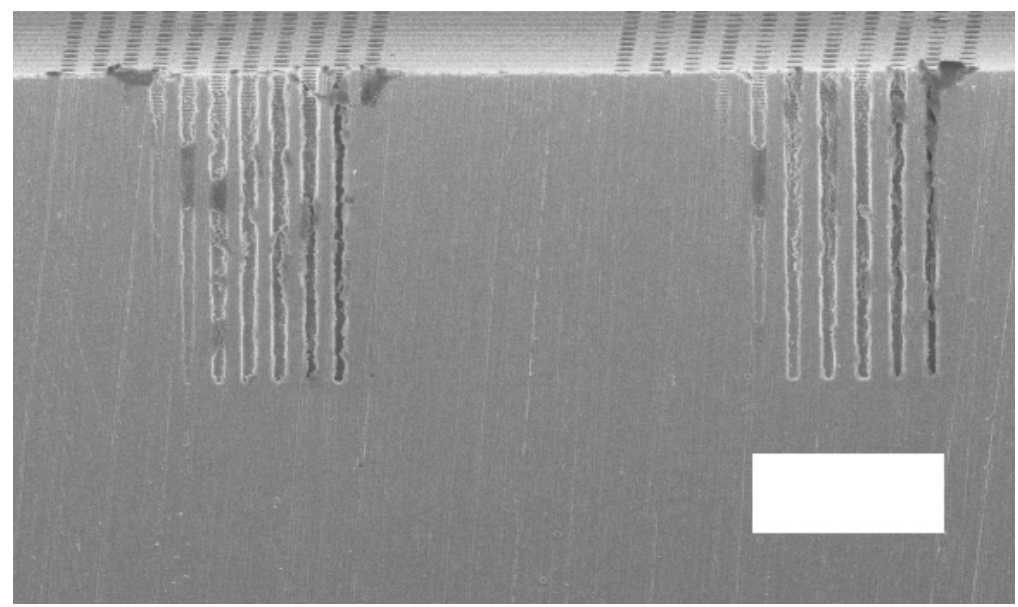

Figure 5.6: Scanning electron micrograph of a cross-section of pores in a polished sample. The scale bar equals $5 \mu \mathrm{m}$. The cross-section was polished using a tripod polisher. Photoresist was removed by calcination. The cross-section surface is flat, without craters. The polished surface is well-aligned parallel to the axis of the pores. The sidewalls of the pores are intact. Debris is visible in the pores, but the size of the pieces of debris is sufficiently small and the remainder of the cross-section surface is flat, allowing the deposition of mask material and the spin coating of resist. 
structures. On some positions minor damage is present, but these areas are few. The observed minor damage does not influence the ability to deposit mask material and resist on the cross-section surface. Furthermore, by carefully checking the structures of interest during polishing, it is possible to ensure that the polishing result is optimal. Figure 5.6 shows that the polished surface is aligned parallel to the axis of the pores and that the sidewalls of the pores are completely intact. There is some debris visible in the pores, which was determined by Energy Dispersive X-ray analysis to contain aluminium-, phosphor-, and possibly silicon- and calcium atoms. The debris is suspected to originate from the lapping film and we are currently investigating how to prevent this debris from collecting on the sample during polishing. However, currently the size and shape of the debris is sufficiently small and flat to allow the deposition of mask material and consecutive spin coating of resist.

\subsection{Alignment of samples in the holder wafer}

In order to test whether a diced sample of $26 \times 0.5 \times 0.5 \mathrm{~mm}^{3}$ can be properly aligned and fixed, several dummy sample pieces were mounted in a holder wafer. These dummy samples do not contain any etched nanopores, but were diced out of an unpatterned $100 \mathrm{~mm}$ silicon wafer glued to UV foil, using a Disco DAD-321 dicing saw equipped with a NBC-Z-2050 blade. After sawing and removal of the foil, the samples were cleaned by immersion in acetone and subsequent heating in an oven $\left(500{ }^{\circ} \mathrm{C}\right.$ for $\left.10 \mathrm{~min}\right)$.

The holder wafer as prepared in section 5.2 was placed on top of a dummy wafer. The samples were placed in the slots of the holder wafer. The surface of the sample was placed in contact with the dummy wafer. In this fashion the dummy wafer accurately aligns the surface of placed samples with the surface of the holder wafer. Two or three layers of photoresist MESA $^{+}$ cleanroom, Olin 908/35) were spin coated at $3000 \mathrm{rpm}$ for $45 \mathrm{~s}$ immediately after each other on a separate double side polished $100 \mathrm{~mm}$ silicon wafer. This yields a so-called "glue" wafer which is then placed on top of the holder wafer. Both wafers were pressed together by a $504 \mathrm{~g}$ weight. The photoresist was hardened overnight in an oven at $50{ }^{\circ} \mathrm{C}$ to ensure a solid connection between samples, holder wafer, and "glue" wafer. After flipping the entire construction over, the dummy wafer was removed. The surfaces of the holder wafer and of the samples therein were cleaned by exposure to oxygen plasma from a Tepla 300E dry etcher for 5 minutes, see Table 5.3.

Figure 5.7(A) is a photograph of an area of the top surface of a holder wafer 
Chapter 5. Fabrication and testing of the holder wafer
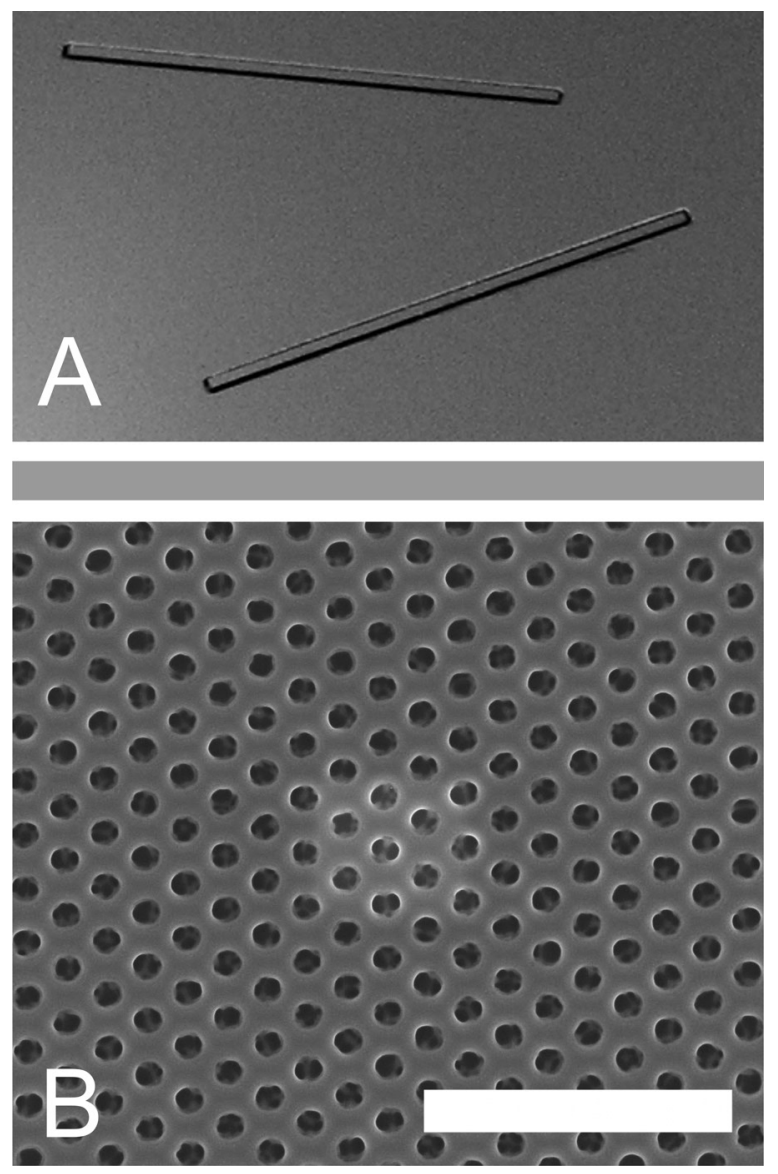

Figure 5.7: A) A photograph of the top surface of a holder wafer with 2 dummy samples glued in the slots. The alignment error of the dummy samples with respect to the holder wafer surface was better than $0.1^{\circ}$. B) Scanning electron micrograph of a pattern in a chromium layer that was deposited on a dummy sample in a holder wafer. The scale bar equals $3 \mu \mathrm{m}$. The pattern is a centered rectangular lattice with intended lattice parameters $a=680 \mathrm{~nm}$ and $c=481 \mathrm{~nm}$. The diameter of the holes was intended to be $D_{\text {mask }}=230 \mathrm{~nm}$. The pattern was milled with a focused ion beam. The quality of the milled holes is excellent, showing that it is possible to prepare a mask on a sample mounted in a holder wafer. 
Table 5.3: Overview of settings used during cleaning of the wafer surface using oxygen plasma from a Tepla $300 \mathrm{E}$ dry etcher.

\begin{tabular}{|l|c|}
\hline Setting & Value \\
\hline Plasma power & $300 \mathrm{~W}$ \\
\hline $\mathrm{O}_{2}$ flow (mass flow controller) & $50 \%$ \\
\hline Pressure & 1.25 mbar (typically) \\
\hline
\end{tabular}

with 2 dummy samples placed in the slots ${ }^{3}$. A "glue wafer" attached to the bottom of the holder wafer and the dummy samples by photoresist ensured a solid attachment of the components, see Figure 5.1(C). The surface of several dummy samples were measured to be parallel to holder wafer surfaces within an accuracy $\gamma<0.1^{\circ}$, which is better than the limit defined in chapter 2 .

\subsection{Mask fabrication on samples mounted in a holder wafer}

In this section we show, as a proof of principle of mask preparation on a holder wafer, how a pattern in chromium was obtained. Following reference [195], we used focused ion beam milling to prepare a metal mask for a photonic crystal structure. An advantage of using a focused ion beam setup is that it allows fast milling of any pattern on the surface. A disadvantage is that only a small area can be processed. Using electron gun evaporation on a Balzers BAK 600A, a $50 \mathrm{~nm}$ thick chromium layer was successfully deposited on a construction of "glue" wafer, holder wafer, and dummy samples mounted in the slots. Subsequently, the chromium layer was patterned using focused ion beam milling, which requires conduction to remove charges away from the milled area. Unfortunately there is only limited conductivity between the sample in the slot and the holder wafer. Therefore a droplet of silver conductive adhesive paste (Alfa Aesar, product number 42469) was applied to improve the electrical connection between these two components. In addition, since the photoresist between the "glue" wafer and the holder wafer is insulating, a separate droplet of silver conductive adhesive paste was applied to ensure conductance between these two wafers. A FEI "xT Nova Nanolab 600" workstation was used to mill the pattern in the chromium layer using a stream file. The gallium ion beam of the workstation was accelerated at $30 \mathrm{kV}$ and the beam current was set at $93 \mathrm{pA}$. To image the

\footnotetext{
${ }^{3}$ Figure 5.7(A) was photographed with a Canon PowerShot A460 AIAF.
} 
patterned sample, the scanning electron imaging functionality of the focused ion beam workstation was used. The high voltage was set at $5.00 \mathrm{kV}$ at a current of $98 \mathrm{pA}$. The working distance was $5 \mathrm{~mm}$ and the Everhart-Thornley secondary electron detector (ETD) was used to record the image.

The pattern chosen for this experiment is a centered rectangular lattice with intended lattice parameters $a=680 \mathrm{~nm}$ and $c=481 \mathrm{~nm}$, see chapter 2. Applying these lattice parameters should yield a photonic crystal with a broad photonic band gap. The diameter of the holes was intended to be $D_{\text {mask }}=230 \mathrm{~nm}$. Figure $5.7(\mathrm{~B})$ shows that milling of the pattern was successful. The Figure shows that the chromium layer has been completely penetrated and that the holes in the mask are

a) nicely circular,

b) homogeneous in size, and

c) uniformly distributed over the surface. The high quality of the milled holes proves that it is possible to prepare masks on a sample mounted in a holder wafer. This method can be used to quickly pattern a sample that already has a first set of pores etched. In the future, the current sample will be used to investigate whether the holder wafer construction allows pores to be etched through the chromium mask using reactive ion etching.

\subsection{Conclusions}

The successful fabrication and testing was described of the holder wafers. The experimental methods was outlined in detail with which the holder wafer was fabricated. We showed how slots could be cut in the holder wafer in which samples can be placed with good alignment accuracy. To be able to deposit mask material and resist, good alignment of the sample surface with respect to the wafer surface is essential. Furthermore, the cross-section surface of the samples needs to be smooth enough to be able to do lithography on them. To polish samples that contain nanopores, the etched structures have to be stabilized using hardened photoresist. We showed that a) this stabilization method works and b) that we are able to remove the photoresist from the structure afterwards. We showed that by "wedge polishing" a flat surface was obtained. As a test, a dummy sample was placed into slots of a holder wafer. The parallel alignment of the dummy sample surface with respect to the surface of the holder wafer was excellent $\left(\gamma<0.1^{\circ}\right)$. The samples were solidly attached to the holder wafer by means of the "glue" wafer, ensuring good mechanical stability. Chromium was deposited and a pattern was successfully milled in the chromium layer on the surface of the dummy sample with a focused ion beam. To summarize, we showed that the 
5.6. Conclusions

holder wafer procedure is suitable for further processing of samples. 



\section{Focused ion beam milling of nanocavities in artificial opals}

\subsection{Introduction}

\subsubsection{Optical cavities in photonic crystals}

Several exciting opportunities arise if the shape and morphology of individual colloidal particles can be controlled. Firstly, a monolayer of colloids can be used as a lithographic opal mask [196]. Fabricating an aperture in one sphere in the monolayer adds a finely controlled feature to the mask. If size, shape and position of the aperture in the mask can be controlled with nanometer precision, detailed structural flexibility beyond the structure of the colloids is obtained. Secondly, if opals are made chemically selective by covering the opal with self-assembled monolayers [80, 81], a cavity inside one sphere will add size-selectivity, since only molecules that fit in the cavity will enter and bind to the walls of the cavity. Additional local chemical specificity can be introduced at the modified nanostructure by local deposition methods (by e.g. ion beams). Thirdly, if an opal is used as a photonic crystal, a single modified colloidal particle in the opal is expected to act as an optical cavity [15]. Obtaining an optical cavity is the goal of our research in this area.

An optical cavity in a photonic crystal can be manufactured by either adding or removing high-refractive index material, which yields a donor or an acceptor, respectively [15, 73]. Figure 6.1 shows an illustrative example of a material cavity (acceptor) consisting of a vacancy at the surface of a $3 \mathrm{D}$ opal photonic crystal.

The essential parameters of optical cavities are the quality factor $Q$ that describes how long light is contained in the cavity, and the mode volume $V_{\text {mode }}$ that describes how strongly the light is confined. Ideally, $Q$ is elevated and $V_{\text {mode }}$ is much smaller than a wavelength cubed [17]. Since theory 


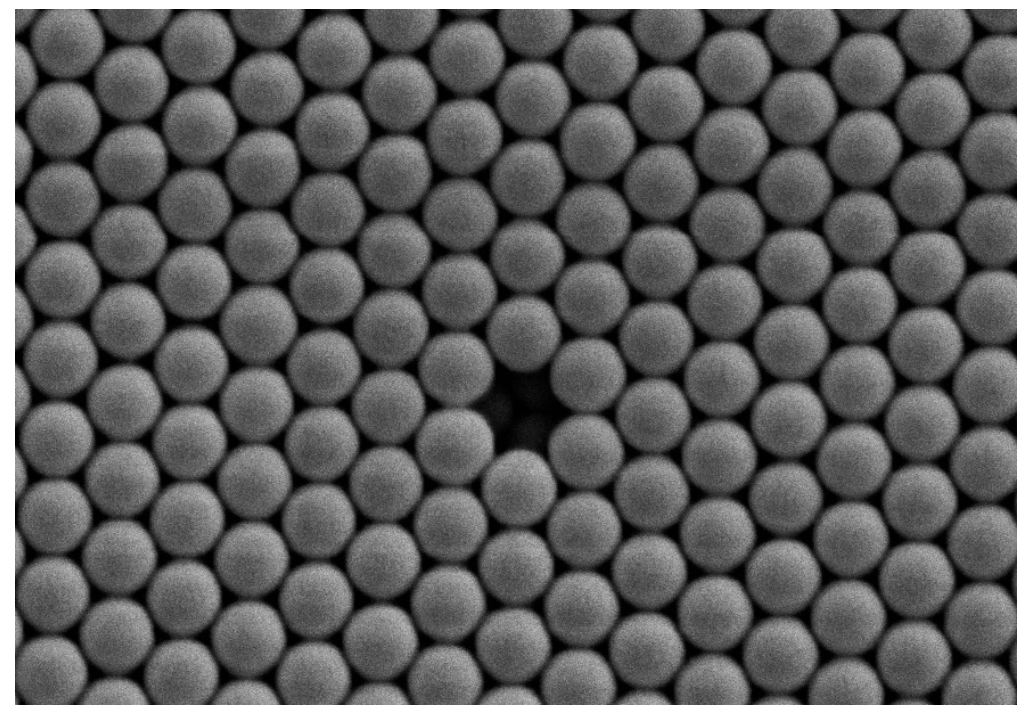

Figure 6.1: Scanning electron micrograph of the surface of a polystyrene artificial opal. A surface cavity consisting of a vacancy is apparent that is observed by chance in a self-organizing opal. The spheres have a radius of $439 \mathrm{~nm}$.

predicts that $Q$ increases with the size of the crystal [73], it is important to incorporate an optical cavity in an extended 3D crystal, such as an (inverse) opal. Since theory predicts as well that a small $V_{\text {mode }}$ is achieved by a point defect with a small physical volume [73] it is crucial to have nanometer process control over the fabrication of a point defect. Obviously, after fabrication it is also vital to be able to locate a single nanocavity or an array of nanocavities in a 3D crystal. Therefore, cavities that appear by chance such as the one in Figure 6.1, do not fulfill this criterion. Recently, there have been several interesting reports on the fabrication of optical cavities in photonic crystals $[85,86,197,198]$. To the best of our knowledge, however, no method has yet demonstrated the fabrication of a single defect or an array of defects at well-defined positions in thick 3D photonic crystals combined with controllable defect-volumes.

\subsubsection{Embedding a cavity in 3-dimensional artificial opals}

There are basically two possible routes to obtain individual modified particles in opals. The first route is to modify individual particles and move them to their desired position on the opal. Moving and placing such particles with nanometer precision is quite challenging and requires the use of, 


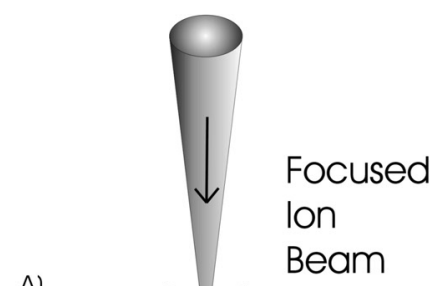

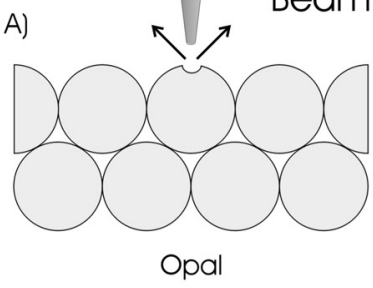

D)

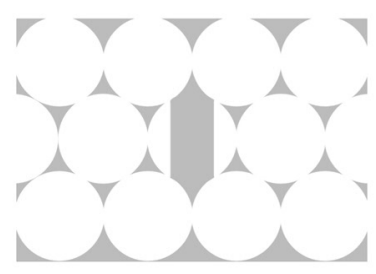

Inverse opal with a cavity inside
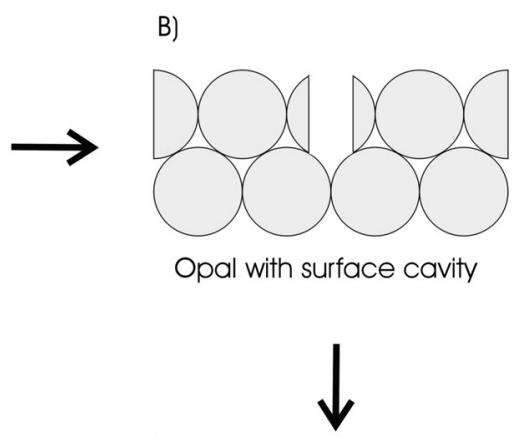

C)

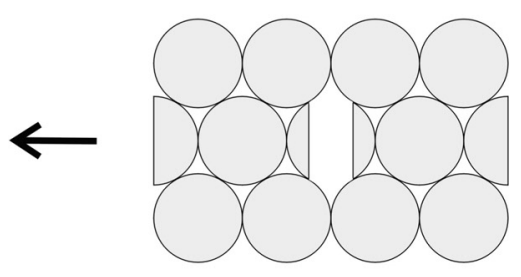

Cover the cavity

Figure 6.2: : Schematic representation of the fabrication of a nanocavity in an inverse opal. A) Material is milled away with nanometer precision with a focused ion beam. (B) A cavity is achieved in the surface plane of the opal. (C) New layers of colloidal spheres are deposited on top of the crystal to bury the cavity in the bulk. (D). The opal and the embedded cavity are inverted to yield an inverse opal photonic bandgap crystal with a point defect. In this scheme, the cavity is formed by additional high index material that fills the cavity formed by the focused ion beam milling process, thereby creating a donor.

e.g., robots or atomic force microscopes $[66,199]$. The second route is to modify a single colloid a posteriori in an already grown artificial opal. Here we describe a versatile method to modify the structure of targeted individual silicon dioxide colloidal particles on the surface of opals using focused ion beam milling (FIB).

Our process to obtain nanocavities for light, outlined in Figure 6.2, combines well-established photonic crystal preparation methods with focused 
ion-beam etching techniques [200]. In the first step (A), a cavity is milled in one of the colloids on the surface of the opal. This results in an opal with a material cavity (B), whose volume is controlled by the focused ion beam parameters. The surface defect is buried in bulk crystal by growing additional crystal layers on top of the surface layer (C), whereby the surface layer acts as a template for subsequently deposited colloidal particles. At this stage the point defect is an acceptor in the opal photonic crystal. To obtain a cavity in a photonic band gap crystal, the opal with defect is turned into an inverse opal (D) by infiltrating all void space with a high refractive index material and subsequently removing the original opal material. To make inverse opals, many ways have been reported [30, 39, 40, 201]. In this chapter we concentrate on steps (A) and (B). In addition, the interaction of light with nano-cavities in the surface of artificial opals, fabricated by focused ion beam milling, is studied by optical microscopy.

\subsection{Experimental methods}

To grow artificial opals we used silicon dioxide or polystyrene colloidal spheres. The silicon dioxide colloids were synthesized with the Stöber method $[46,202]$ or from micro emulsions [22, 203]. They had radii $R=113 \mathrm{~nm}$ (micro emulsion), $231 \mathrm{~nm}$, or $343 \mathrm{~nm}$ (both Stöber), and size polydispersity $\Delta$ $=1 \%, 4 \%$, and $6 \%$ respectively. The polystyrene spheres were purchased from Duke Scientific. They had radii $R=111 \mathrm{~nm}$ or $241 \mathrm{~nm}$, and size polydispersity $\Delta=2 \%$ and $1 \%$ respectively. The photonic crystal opals were grown in $300 \mu \mathrm{m}$ thick glass capillaries under the influence of gravity, yielding relatively thick opals [204], or by the vertical controlled drying method of Jiang et al., yielding thinner opals [27].

Prior to milling, carbon was deposited on the surface of the opal with a Polaron SEM coating system operating at a current of 16 A, or with a BioRad E6700 Turbo Coater operating at a current of 50 A. Carbon layers have typical thicknesses of about $10 \mathrm{~nm}$ and assist in removing charges from the milled area. The carbon layers were removed after the milling experiments using oxygen plasma from a Nanotech PlasmaPrep 100 with typical parameters: 1 minute duration, $60 \mathrm{~W}$ forward power, 2 mbar pressure, $50 \mathrm{sccm} \mathrm{O} \mathrm{O}_{2}$ flow, and substrate temperature $150{ }^{\circ} \mathrm{C}$. In recent experiments the carbon layers were removed using oxygen plasma from a Tepla $300 \mathrm{E}$ with typical parameters: 15 minutes duration, $300 \mathrm{~W}$ plasma power, 1.25 mbar pressure, and $50 \% \mathrm{O}_{2}$ flow.

Defects and arrays of defects were milled using a FEI "FIB 200" workstation, using a gallium ion beam accelerated to $30 \mathrm{kV}$ and a beam current of 1 
$\mathrm{pA}$. The focus of the ion beam has a diameter of about $10 \mathrm{~nm}$. The colloidal spheres were milled with the ion beam by writing square patterns of $50 \times 50$ $\mathrm{nm}^{2}$ or $80 \times 80 \mathrm{~nm}^{2}$, positioned on the center of the sphere. Total milling times were varied from $5 \mathrm{~s}$ up to a maximum of $30 \mathrm{~s}$. In addition the cavities were milled intermittently. This means that after about every $5 \mathrm{~s}$, milling was stopped for a short period (typically a few seconds). Together with the applied carbon layers, the intermittent procedure promotes redistribution of charges away from the processed area, preventing charge build-up of the milled area which typically results in beam drift and substantial damage to the opal. Before each new milling step, the square pattern used for milling was repositioned on its original position on the milled colloidal sphere. In this manner an array of cavities was milled successfully. In a number of experiments milling was gas-enhanced by iodine vapor [205]. This adds a chemical etching component to the process and is normally used to improve aspect ratios of milled features.

Arrays of single defects were also milled using a newer FEI "xT Nova Nanolab 600" workstation, using a gallium ion beam accelerated to $30 \mathrm{kV}$ and a beam current of $9.7 \mathrm{pA}$. The focus of the ion beam had a diameter of about $20 \mathrm{~nm}$. The defects were milled with the ion beam by writing circular patterns with a diameter of $120 \mathrm{~nm}$, positioned on the center of the sphere. Arrays were milled either in one sweep or milled intermittently with approximately $10 \mathrm{~s}$ between each step. All single defects which were part of the array were milled in parallel. To image the samples in-situ, the imaging functionality of the FEI workstation was used. All other pictures where imaged separately with a LEO 1550 high-resolution scanning electron microscope, operating at a low acceleration voltage ( 1 to $1.5 \mathrm{kV})$, a working distance of $3 \mathrm{~mm}$ and using the inlens detector to record the images.

Optical microscopy experiments were performed using a Nikon Eclipse ME600L optical microscope, equipped with 5x, 10x, 20x, 50x, and 100x CFI LU PLan BD objectives and a CFI 10x eyepiece. The objectives allow for bright-field and dark-field illumination of the samples. The optical micrographs shown in this chapter were imaged using the CFI LU PLan BD 100x objective, which has a numerical aperture of $N . A .=0.90$ and a working distance of $1 \mathrm{~mm}$. The high numerical aperture corresponds to a cone of light through the objective with an angle of $128^{\circ}$, which means that many wave vectors are probed. A DXM-1200F high-resolution digital camera is equipped to this microscope to record the images. 


\subsection{Milling results}

\subsubsection{Milled defects and arrays of defects}

Figure 6.3(A) shows a high-resolution scanning electron microscopy image of three silicon dioxide spheres on an opal, milled with a square pattern of 50 x $50 \mathrm{~nm}^{2}$ using the FEI "FIB200" workstation. The spheres have a radius $R=231 \mathrm{~nm}$. The left sphere was milled for $5 \mathrm{~s}$, the middle sphere for 10 $\mathrm{s}$ and the right sphere for $15 \mathrm{~s}$. All spheres were milled intermittently with steps of $5 \mathrm{~s}$ each. The milled defects are nearly circular in shape, confirming that beam- and stage drift during milling were small. It seems that milling a square pattern with a higher current of $4 \mathrm{pA}$ yields a square shaped defect, suggesting that at higher milling currents the structural definition of the pattern is improved. However, in these experiments our crystals were easily destroyed since particles were expelled from the surface, resulting in large craters. Therefore milling at this current could not be studied further. The milled cavities are very near to the centers of the colloidal spheres, where we intended to make them. The Figure clearly demonstrates that nanocavities were milled successfully.

Figure 6.3(B) shows an array of defects milled with the FEI "xT Nova Nanolab 600" workstation on an opal grown by the vertical controlled drying method on a glass substrate [27]. The opal consists of silicon dioxide spheres with a radius $R=113 \mathrm{~nm}$. The defects were milled in parallel using circular patterns $(R=60 \mathrm{~nm})$ for a total of $1.2 \mathrm{~s}$. Milling occurred intermittently with a total of 4 sweeps of $300 \mathrm{~ms}$ each. The defects appear to be almost perfectly cylindrical in shape. On the surface, these defects have a diameter of approximately $130 \mathrm{~nm}$. The defect on the bottom left of the array was slightly displaced and overlaps an interstitial. A complete perforation can be observed for four of the defects, since the spheres in the layer below are visible.

Figure 6.3(C) shows an array of defects milled on the same opal as in Figure $6.3(\mathrm{~B})$. We displaced the pattern and the defects were milled between two spheres instead of on the centre of a sphere. The defects on this sample were milled in parallel in one milling step of 6.3 seconds using circular patterns $(R=60 \mathrm{~nm})$. The defects appear to be conical and have a larger base diameter compared to the cylindrical ones shown in Figure 6.3(B). The larger defect size is explained by the longer milling time. Figure $6.3(\mathrm{C})$ shows that it is possible to mill defects in-between two touching spheres.

To analyze the structure of the milled features, the sizes of the milled cavities were measured. The cavities are circular on the surface and a white outline of the defect is visible on all scanning electron micrographs. This 


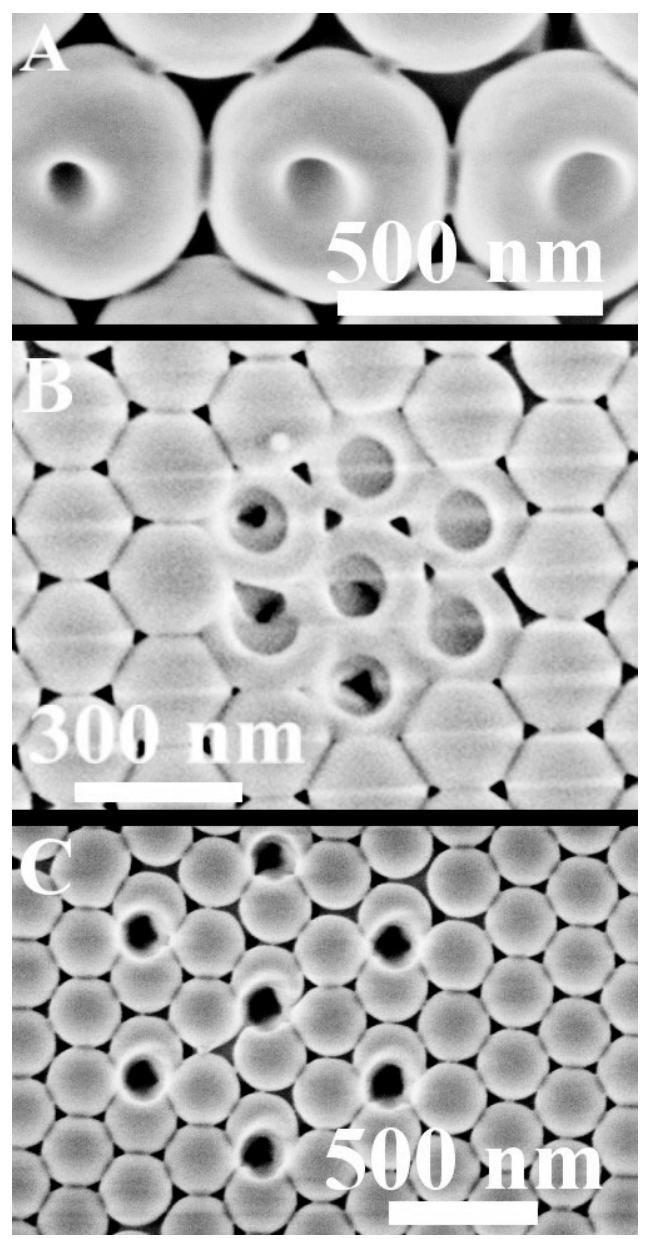

Figure 6.3: (A) Scanning electron micrograph of three colloidal spheres on the surface of an artificial opal of silicon dioxide colloidal spheres. The spheres are milled intermittently with a $50 \times 50 \mathrm{~nm}^{2}$ square pattern. The left sphere was milled for $5 \mathrm{~s}$, the middle sphere for $10 \mathrm{~s}(2 \times 5 \mathrm{~s})$ and the right sphere for $15 \mathrm{~s}(3 \times 5 \mathrm{~s})$. (B) Scanning electron micrograph of an array of seven milled material cavities on the surface of an artificial opal of silicon dioxide colloidal spheres. The defects on this sample were milled in parallel for a total of $1.2 \mathrm{~s}$ in 4 sweeps of $300 \mathrm{~ms}$ each, using a circular pattern with a radius $R$ of $60 \mathrm{~nm}$. (C) Scanning electron micrograph of a different array of seven milled material cavities on the surface of an artificial opal of silicon dioxide colloidal spheres. The defects on this sample were milled in parallel for a total of $6.3 \mathrm{~s}$, using a circular pattern with a radius of $60 \mathrm{~nm}$. The pattern was displaced with respect to the centers of the spheres and the defects were milled between two spheres. 
outline originates mainly from charging effects caused by the electron beam on the curved edge of the defect and delineates where the edge approximately starts. Three different values for the sizes of the cavities were estimated: the diameter of the inner edge $D_{\text {inner }}$, of the outer edge $D_{\text {outer }}$, and of the middle of the white outline of the defect $D_{\text {middle }}$. Diameters of all estimates were measured in four directions: the horizontal, the vertical and both diagonals. These values were averaged and one half of the variation between these values was taken as the statistical error on $D_{\text {inner }}, D_{\text {outer }}$, and $D_{\text {middle }}$. The mean values for $D_{\text {middle }}$ and the extremes $D_{\text {inner }}$ and $D_{\text {outer }}$ represent the measured defect size, with an upper bounded error bar. In this way the size of the cavity is effectively represented by its white outline. Measured diameters are compared to the set diameter $\left(D_{\text {set }}\right)$, which is determined by the square pattern used for milling. In this case $D_{\text {set }}=\sqrt{2} \cdot a$, where $a$ represents the length of the side of the square. For example, a square of $a=50 \mathrm{~nm}$ is used to mill a cavity with $D_{\text {set }}=71 \mathrm{~nm}$.

Figure 6.4 shows the measured cavity size versus the total milling time for a number of different cavities: the mean values for $D_{\text {middle }}$ have been plotted and the vertical bars represent the extremes given by $D_{\text {inner }}$ and $D_{\text {outer }}$. The graphs represent milling experiments with different patterns. Figure 6.4(A) shows results for $D_{\text {set }}=71 \mathrm{~nm}$. The data consists of three separate milling experiments on two types of opals. The circles represent experiments on a thick artificial opal $\left(R_{\text {spheres }}=231 \mathrm{~nm}\right)$ and the squares represent an experiment on a thin artificial opal $\left(R_{\text {spheres }}=113 \mathrm{~nm}\right)$. The three experiments with $D_{\text {set }}=71 \mathrm{~nm}$ show good mutual agreement, indicating that:

a) The milling experiments are reproducible.

b) The results are independent of whether the opal is made from small or large colloidal spheres.

c) The results are independent of whether a thick opal grown by sedimentation, or a thin opal grown by the vertical controlled drying method was milled.

d) The milling results are equal, irrespective of whether the gas-enhanced etching procedure is used or not.

The measurements at $t=25 \mathrm{~s}$ differ from each other, for currently unknown reasons. The open circle at $t=20 \mathrm{~s}$ is a suspect data point since the cavity is ellipsoidal due to a faulty milling attempt as a result of strong beam drift. 
A peculiar observation is that for opals from large colloidal spheres the gasenhanced etching procedure yielded unsatisfactory results, whilst for opals from smaller spheres, which are more easily destroyed under standard milling conditions, this procedure seems a key to successfully obtaining material cavities.

Figure 6.4(B) shows the measured data for the $D_{\text {set }}=113 \mathrm{~nm}$ milling experiment on a thick opal. The data shown consists of two separate milling experiments, differentiated by open and closed circles. We observe that for milling times longer than $12 \mathrm{~s}$ the diameter of the defects start to increase compared to $D_{\text {set }}$.

From Figure 6.4(A) and (B) we conclude that with increasing total milling time, the resulting cavities become larger for both selected square patterns and for both types of opals. The structures milled with $D_{\text {set }}=113 \mathrm{~nm}$ start to deviate from $D_{\text {set }}$ for milling times of more than $12 \mathrm{~s}$, while the cavities milled with $D_{\text {set }}=71 \mathrm{~nm}$ already deviate from $D_{\text {set }}$ at $t<5 \mathrm{~s}$. This observation indicates that milling of larger patterns proceeds with more control over the size of the cavity. This may be naively expected because the drift of the ion beam is constant, which means it has a lower relative contribution to the acquired cavity diameter when milling large structures compared to the milling of small structures.

Figure 6.4(C) shows data acquired from an experiment on a newer FEI "xT Nova Nanolab 600" workstation. During these experiments a milling current of $9.7 \mathrm{pA}$ was used. The Figure shows milling results for $D_{\text {set }}=225 \mathrm{~nm}$ on a thin opal for milling times up to $10 \mathrm{~s}$. Similar to the results from Figure 6.4(A) and 6.4(B), the measured cavity diameter increases slightly with milling time at a rate of less than $10 \mathrm{~nm}$ per milled second. The fact that in this setup the diameter increases almost linearly with longer etching times allows for even better control of the defect geometry. Although ten times higher currents were used for milling, the measured diameters do not show larger increases compared to the experiments shown in Figure 6.4(A) and 6.4(B). This result shows that beam drift is still being reduced by the carbon layer on top of the samples.

To investigate how deep colloidal nanoparticles can be milled with a focused ion beam, we have performed additional milling experiments on single spheres on a substrate. Figure 6.5 shows a polystyrene sphere on a silicon substrate after milling $\left(R_{\text {sphere }}=241 \mathrm{~nm}\right)$. It is seen that not only the particle itself is milled, but also the substrate below, see arrow. Therefore we conclude that our focused ion beam milling procedure is not only a surface effect, but that deep material nanocavities are realized, with size aspect ratios of more than 4 . The colloidal particles are effectively turned into 
Chapter 6. Focused ion beam milling of nanocavities in artificial opals

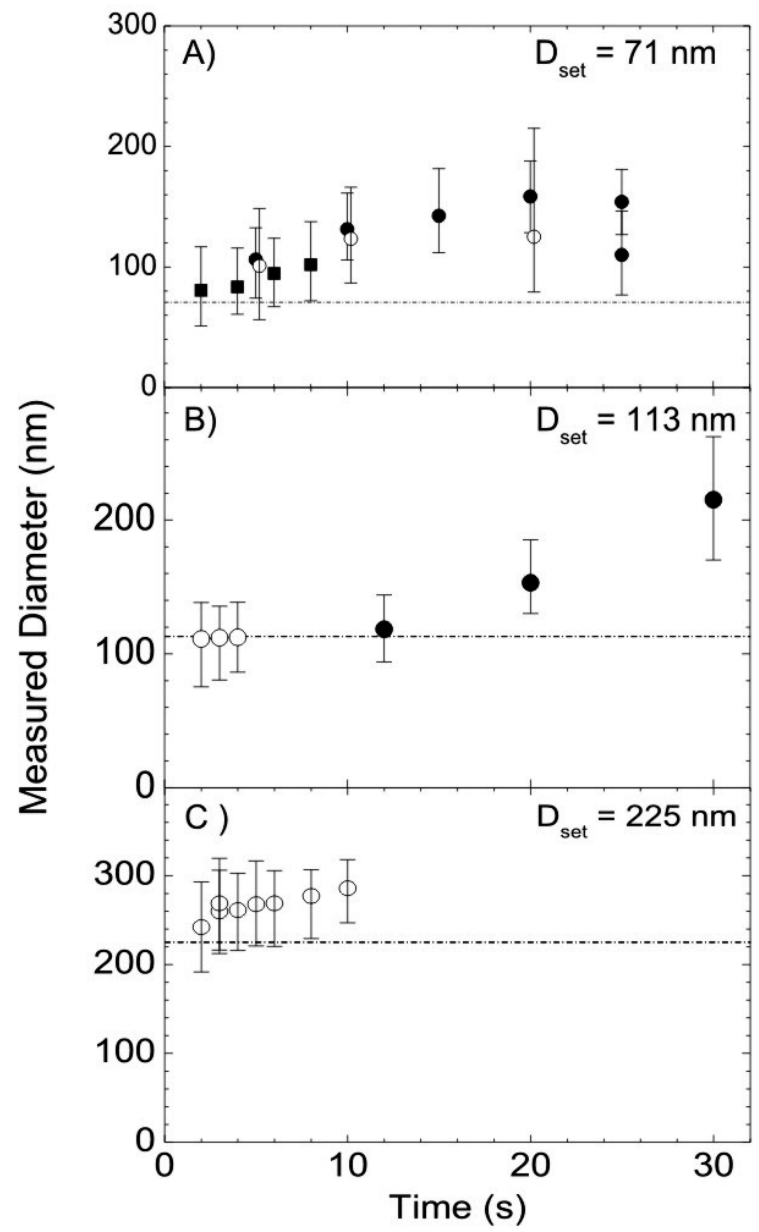

Figure 6.4: Measured cavity diameter as a function of total milling time. The mean values for $D_{\text {middle }}$ have been plotted and the vertical bars represent the extremes $D_{\text {inner }}$, and $D_{\text {outer }}$. The open symbols depict data taken with the FIB in imaging mode and the closed symbols are data from the high resolution scanning electron microscope. The dashed horizontal lines represent $D_{\text {set }}$. (A) Results for $D_{\text {set }}=71 \mathrm{~nm}$. The circles represent experiments on a thick opal $\left(R_{\text {spheres }}=231 \mathrm{~nm}\right)$. The squares represent an experiment on a thin artificial opal $\left(R_{\text {spheres }}=113 \mathrm{~nm}\right)$. For clarity the open circles are slightly offset from $t=5,10$ and $20 \mathrm{~s}$. (B) Data for the $D_{\text {set }}=113 \mathrm{~nm}$ milling experiment on a thick opal $\left(R_{\text {spheres }}=231 \mathrm{~nm}\right)$. (C) Data for the $D_{\text {set }}=225 \mathrm{~nm}$ milling experiment on a thin opal $\left(R_{\text {spheres }}=343 \mathrm{~nm}\right)$ with the FEI "xT Nova Nanolab 600" workstation. 


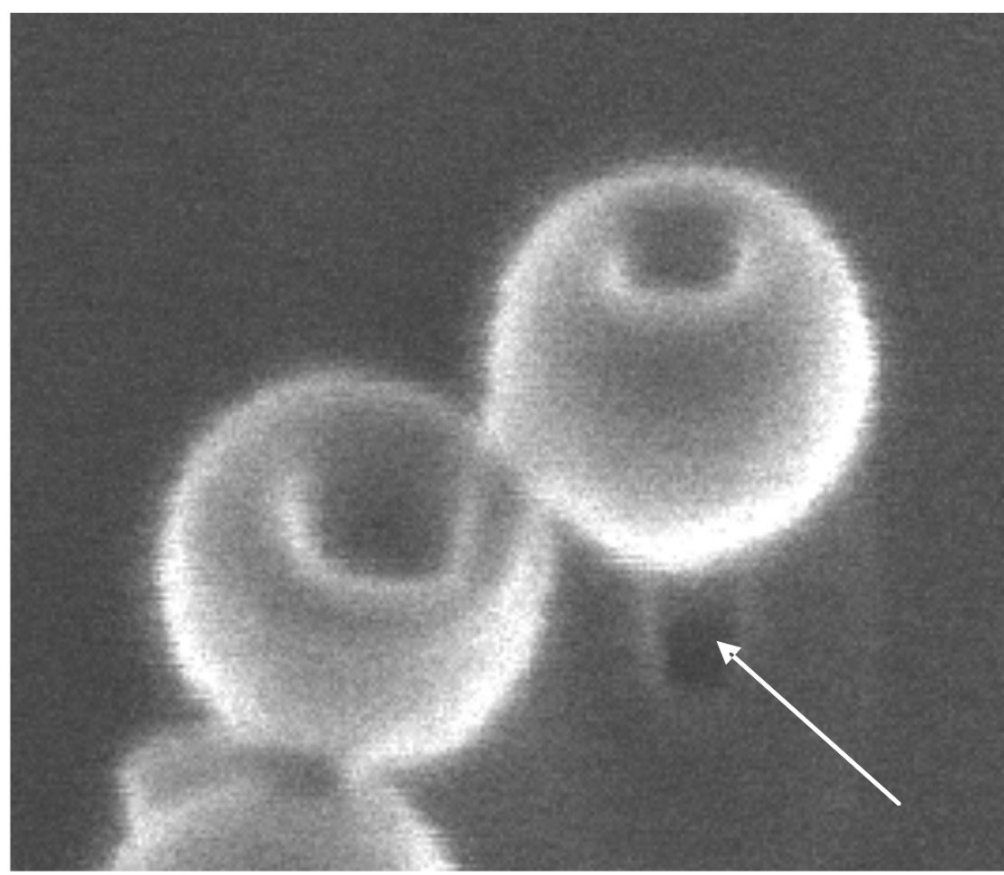

Figure 6.5: Micrograph of a polystyrene sphere $\left(R_{\text {spheres }}=241 \mathrm{~nm}\right)$ on a silicon substrate. The white arrow indicates where milling has occurred in the substrate, showing that the sphere was completely perforated and effectively turned into a nano-bead.

"nano-beads". This result is confirmed by the array shown in Figure 6.3(B).

\subsubsection{Controlling the cavity profile}

Figure 6.6 shows isolated colloidal polystyrene spheres on a silicon substrate milled with two different beam currents and two pattern sizes. Figure 6.6(A) shows the selected spheres prior to milling, and Figure 6.6(B) displays the polystyrene colloidal spheres after milling. Spheres 1 and 2 were milled at $1 \mathrm{pA}$ and spheres 3 and 4 at $4 \mathrm{pA}$. Two square pattern sizes were selected: $100 \times 100 \mathrm{~nm}^{2}$ for spheres 1 to 3 and $250 \times 250 \mathrm{~nm}^{2}$ for sphere 4 .

Figure 6.7 shows the results of the measured relative diameters of the cavities in the milled spheres at the milling currents of $1 \mathrm{pA}$ and $4 \mathrm{pA}$. From the Figure two observations can be derived: a) When polystyrene colloidal spheres are milled at these currents we find that $D_{\text {meas }}$ over $D_{\text {set }}$ is approximately 1 . This means that the cavity sizes are almost equal to the size that can be expected from the square pattern used for milling. b) 


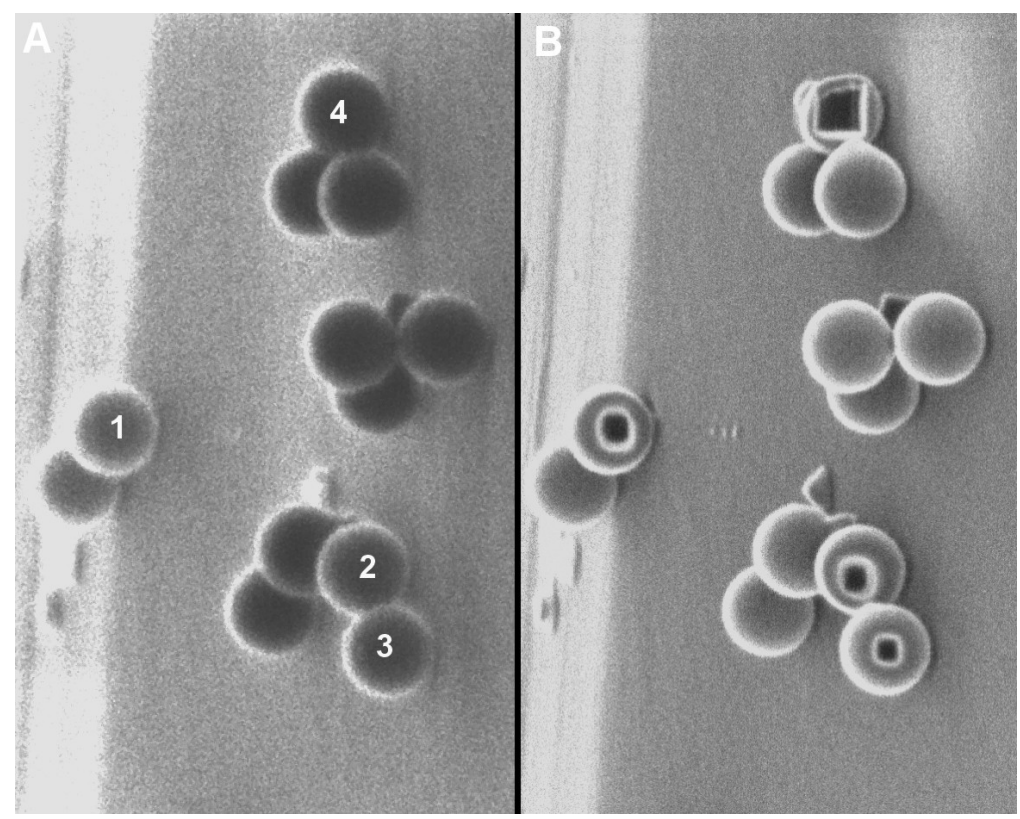

Figure 6.6: Scanning electron micrograph of polystyrene colloidal spheres prior (A) and after milling $(B)$. The spheres were milled with two different currents and two different pattern sizes. The radius of the colloidal spheres is $241 \mathrm{~nm}$.

The difference between $D_{\text {inner }}, D_{\text {outer }}$, and $D_{\text {middle }}$ (vertical bar) is smaller for cavities milled at $4 \mathrm{pA}$, compared to the cavities milled at $1 \mathrm{pA}$. This shows that at $4 \mathrm{pA}$ the width of the white outline is significantly smaller compared to the width at $1 \mathrm{pA}$. This represents a cavity with a sharper edge on the surface and lower tapering, which is explained further by Figure 6.8; it shows a cartoon of a cross-section of two types of cavities in a sphere. The dashed line shows a cavity with a highly tapered profile, corresponding to a cavity milled at a low current. The slope of the edge of the cavity is low, resulting in a broader white outlining of the cavity as depicted by the dashed bar (left side of Figure 6.8). The dotted line represents a cavity with steep sidewalls (low tapering), corresponding to a cavity milled at a high current. The edge of these cavities is very narrow and sharply curved, resulting in a narrow white outline on the scanning electron micrographs (right side of Figure 6.8). We conclude that when processing isolated colloids with a milling current of $4 \mathrm{pA}$ we have better control over the profile of the cavity compared to milling at $1 \mathrm{pA}$. Unfortunately milling with currents exceeding $1 \mathrm{pA}$ was not possible with this apparatus on artificial opal structures due 


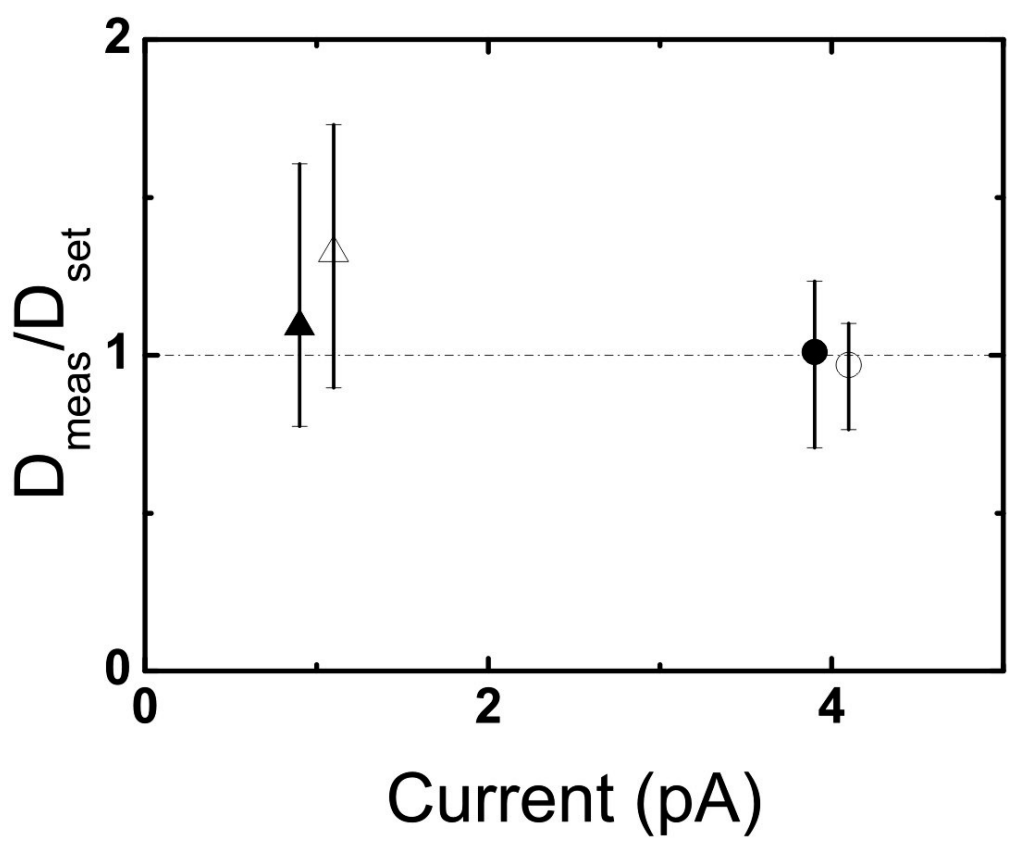

Figure 6.7: Measured cavity size $\left(D_{\text {meas }}\right)$, relative to the set size $\left(D_{\text {set }}\right)$ versus milling current. The filled triangles shows the data for colloidal sphere 1 of Figure 6.6, the open triangles show the data for sphere 2, the filled circles are data for sphere 3, and the open circles for sphere 4. The vertical bar through each point depicts the width of the complete white outlining of the cavity on the scanning electron micrograph. The values are slightly offset from 1 and $4 \mathrm{pA}$ for clarity.

to breakdown of the crystals. We will discuss this further in section 6.3.3.

In Figure 6.6 we observed that the pattern of $250 \times 250 \mathrm{~nm}^{2}$ is milled as a square, while the patterns of $100 \times 100 \mathrm{~nm}^{2}$ are milled into, what appear to be, circular cavities. This indicates that control over the shape of the cavity increases when the milled pattern gets larger.

\subsubsection{Milling with too high currents}

Milling of cavities on the surface of the artificial opals was also attempted at, what turned out to be, too high milling currents. Figure 6.9 shows the result of such an attempt: a crater is formed during the milling process. We do not observe any damaged particles. Therefore we suspect that whole spheres are ejected from the crystal. This is confirmed by the following observation: on the right side of the Figure spheres are shown from which only the top is displayed. Imaging of the Figure occurs from the top down and from 


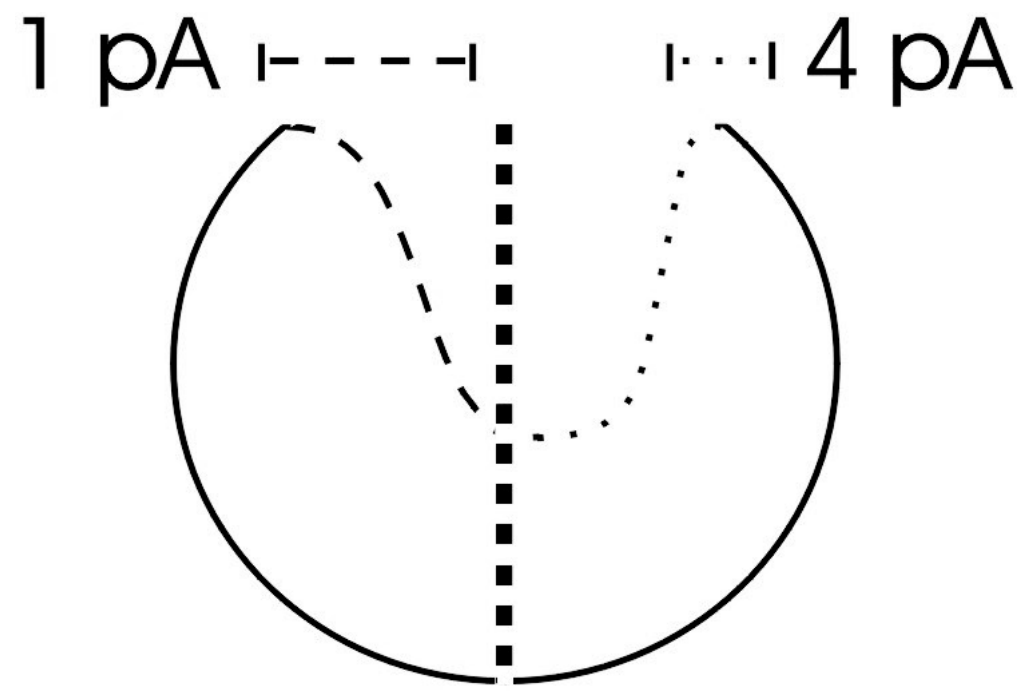

Figure 6.8: Schematic cross-section of cavities in a sphere at low and high beam currents. The dashed line (left-side) shows a cavity with a highly tapered profile. The dotted line (right-side) represents a cavity with little tapering: the sidewalls are more vertical to each other and the edge on the surface of the cavity is very narrow and sharply curved. We propose that the profile difference is represented in the width of the white outline in the scanning electron micrographs.

left to right. Apparently, during imaging, individual colloidal spheres are ejected from the crystal and these spheres are no longer present on the crystal during the remainder of the imaging step. It is a coincidence that in this particular case only spheres on the right side of the imaged area were ejected; spheres removed from other areas on the image have been observed in other experiments. From the observations above we conclude that during imaging and milling at $4 \mathrm{pA}$ the charge build-up on this sample is so significant that the repulsive Coulomb forces between spheres on the surface and the crystal are sufficiently large to overcome binding forces. The colloidal spheres are ejected from the crystal, resulting in a large crater. We observed the same phenomenon when trying to mill material cavities in thin polystyrene artificial opals. Opals made from small spheres are less stable during milling when compared to opals made from larger spheres. These smaller colloidal spheres have a smaller interparticle distance and experience stronger repulsive Coulomb forces. They are expelled more easily from the crystal. Possibly, in the case of opals made from smaller spheres, the vapor entered in the milling chamber during the iodine enhanced etching method 


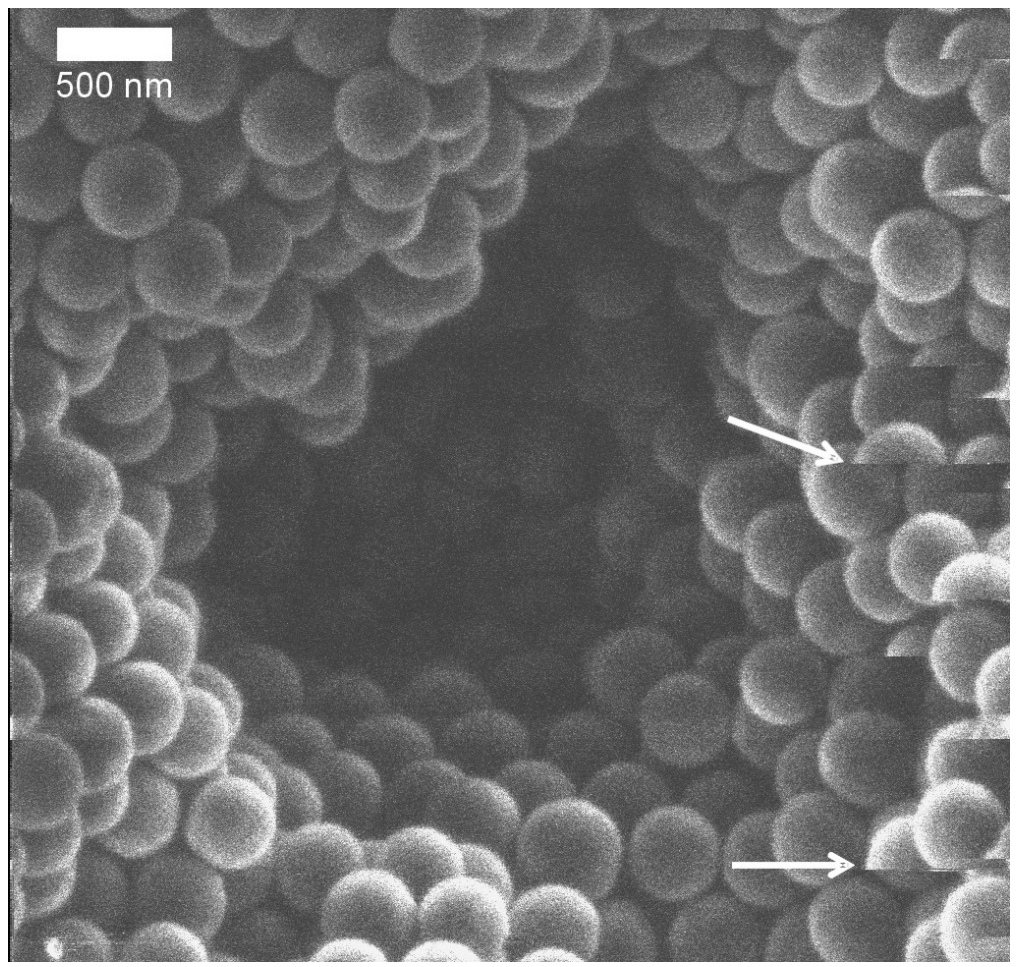

Figure 6.9: Scanning electron micrograph of an attempt to mill a square on the surface of a thick silicon dioxide artificial opal with a too high current. The scale bar represents $500 \mathrm{~nm}$. The arrows point to spheres which were only imaged in part, because they were ejected from the crystal during imaging.

assists in removing charges from the milled surface, thus preventing damage to the crystal. It remains a question why in the case of the opals made from larger spheres, the gas-enhanced method has an adverse effect on the obtained results.

\subsection{Discussion}

An important result is that the size of the milled defects increases with the total milling time, see Figure 6.4. This result is probably due to the small amount of beam drift caused by the continuous charging of the sample during milling, which expands the boundaries of the milled area in time. The increase of defect size could also be due to the variations while repositioning the writing pattern on the milled cavity during every intermittent milling 
step, which is inherent to the applied method.

We observed that milling of larger patterns proceeds with more control over the size of the cavity. This improved control is attributed to a) a lower apparatus dependent contribution of beam drift and b) a lower average gallium ion density in the case for milling experiments with larger writing patterns. A lower ion density results in less charge on the milled sphere and therefore reduces the contribution of charge induced beam-drift in these cases.

In Figure 6.3(B) we obtained cylindrical defects with a diameter close to the intended diameter. During this experiment we milled intermittently, which means that the charging of the surface due to the bombardments with ions was strongly reduced. This resulted in less charge induced beamdrift. The reduced beam drift may well be responsible for obtaining this result, which is much better when compared to the result from Figure 6.3(C). There the intermittent procedure had not been applied and the defects are conical in shape. Milling intermittently appears to be very important for obtaining a successful milling result; an observation which was confirmed by our experiments on both FIB workstations used.

In reference [206] it is stated that high-resolution applications of focused ion beam milling are in a "relatively less developed state". This is confirmed by our experience that milling of the cavities turned out to be unexpectedly challenging when compared to established focused ion beam milling methods [200]. The challenges are due to the combination of the very small size of the feature to be milled, the non-conductive opal substrate, and the substrate being built of loose elements, i.e., the colloids, which repel each other when charged too much by the ions, thereby destroying the artificial opal.

\subsection{Scattering of light by nano-cavities}

Material cavities on the surface of an opal scatter light, somewhat similar to a small aperture in an opaque screen ("Babinet's principle") [207]. In order to investigate the interaction of light with such material cavities, we have performed optical microscopy experiments on missing spheres on the surface of a polystyrene opal. In addition we observed scattering of light at a nano-cavity at the surface of a silicon dioxide artificial opal that was fabricated with a focused ion beam workstation ${ }^{1}$.

Figure 6.10(A) shows an optical micrograph of a surface cavity formed by a missing sphere, under dark-field white-light illumination. Figure 6.10(B)

\footnotetext{
${ }^{1}$ This work was done in collaboration with B.H. Husken.
} 


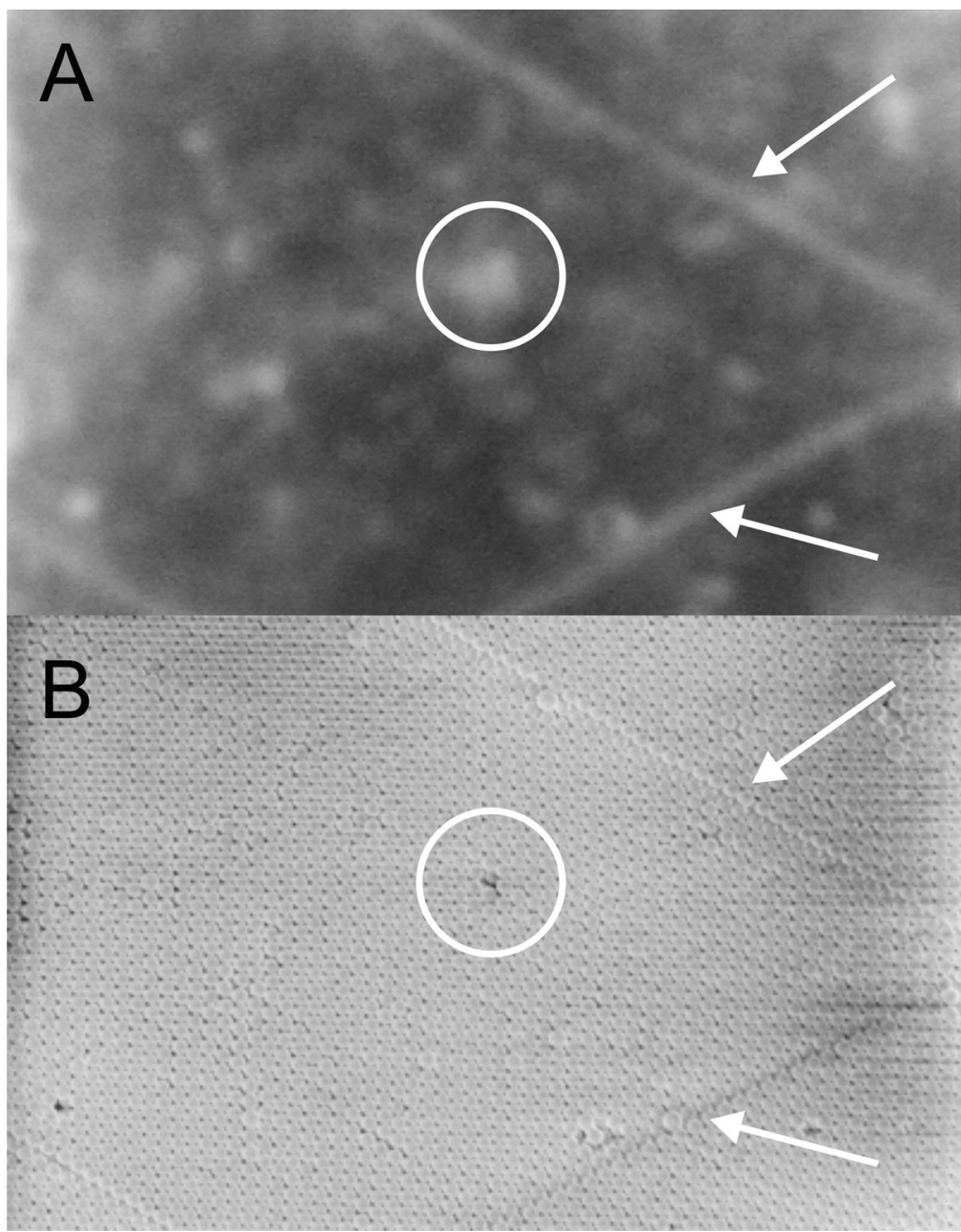

Figure 6.10: A) Dark-field optical micrograph of the surface of a polystyrene artificial opal with a missing sphere after conversion to 8-bit grayscale and increasing the contrast. B) Scanning electron micrograph of the exact same position. The radius of the spheres is $111 \mathrm{~nm}$. Encircled is a white spot of light scattering from the missing sphere. The spot appears exactly appears at the position of the missing sphere, as can be confirmed by the steps in the artificial opal which are indicated by the arrows. 
shows a scanning electron micrograph of the same region. It is an exciting result how well the detailed features in the optical and in the scanning electron micrographs can be correlated: clearly visible in the optical micrograph is the white spot (encircled in white) as a result of light that scatters from the cavity that is also seen by scanning electron microscopy. Optical bright-field images also revealed a bright spot at the position of the cavity, indicating that light that is incident from completely different directions than in Figure 6.10(A) also scatters towards the detector. These optical results confirm that such small cavities interact with light over a broad range of wave vectors, as expected. Both optical and scanning electron micrographs also reveal a clear step on the surface that also scatters light. These steps are used to confirm that the bright white spot in the optical micrograph is indeed originating from the same position as the missing sphere.

A nano-cavity for a single mode of light must have a smaller size than the size of a single (missing) sphere and the size of the nano-cavity needs to be optimized. To this end we investigated the fabrication of nano-cavities in this chapter. The microscopy results of one such cavity is shown in Figure 6.11(A), which shows an optical micrograph from an array of intentional defects, under dark-field white-light illumination. Figure 6.11(B) shows a scanning electron micrograph of the same region. Here also the detailed features in the optical and in the scanning electron micrographs can be accurately correlated: in the optical micrograph, the brighter spot is clearly visible in the white circle as a result of light that scatters from the array of defects that is also seen by scanning electron microscopy, see Figure 6.11(B) and inset. To the best of our knowledge, this is the first time that such small individual structures are identified in three-dimensional photonic crystal structures that also clearly interact with light. This result indicates that light that is incident from within the horizontal plane of Figure 6.11(A) scatters towards the detector by the fabricated nanostructure. The domain boundary which has the same shape in both Figures (A) and (B) confirms that the brighter spot on the optical photograph appears on the position of the array of defects. Optical bright-field images also revealed a feature at the position of the array of defects, showing that the defect interacts with light incident on the sample under different angles when compared to the dark-field experiments. While these defects obviously form very leaky optical cavities we observe that such small defects interact with light over a broad range of wave vectors as is ultimately of interest for optical cavities. Detailed and spectroscopic information can be obtained by, for instance, advanced near-field scanning optical microscopic techniques [208]. 


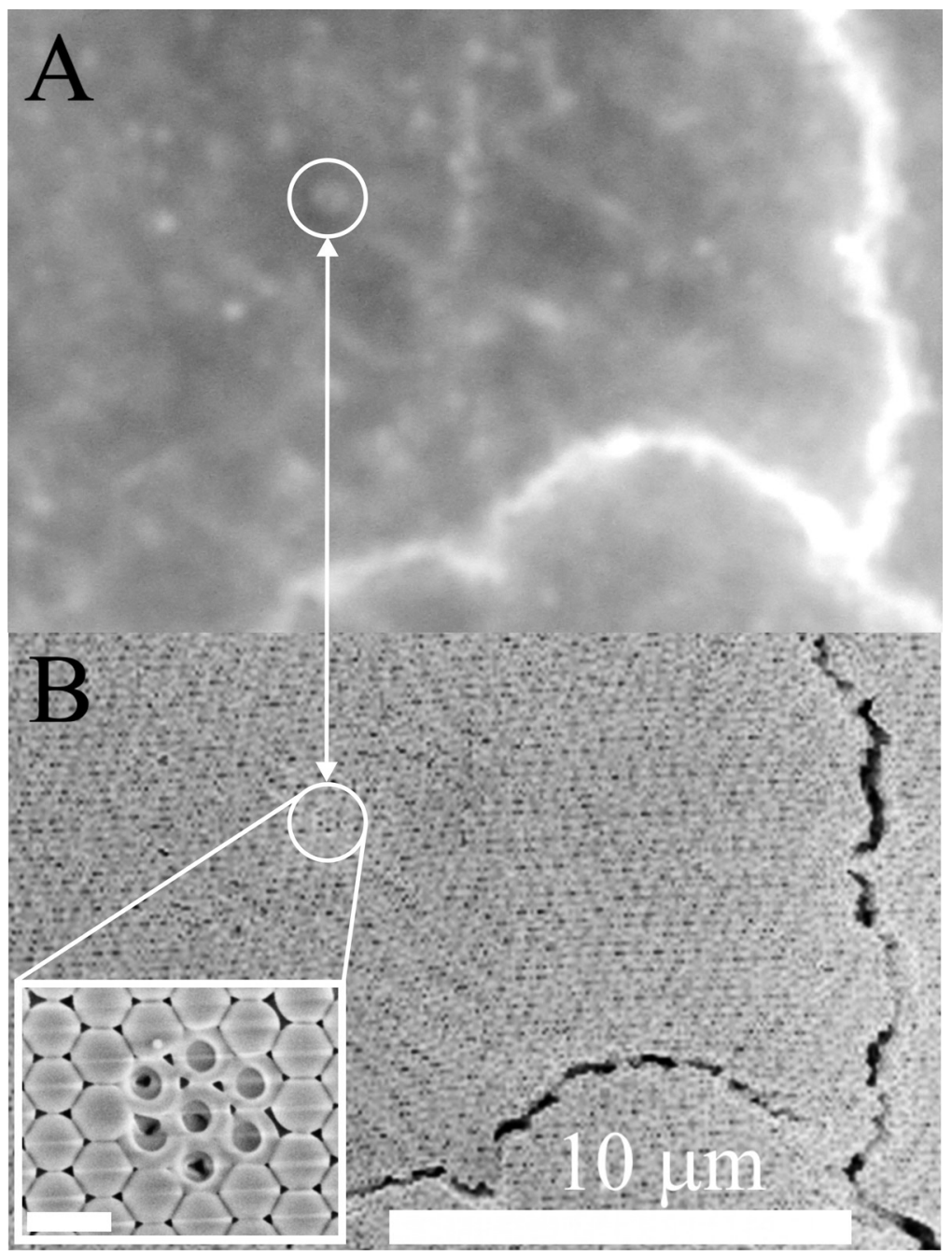

Figure 6.11: A) Dark-field optical micrograph of a silicon dioxide artificial opal with an array of defects after conversion to 8-bit grayscale and increasing the contrast. (B) Scanning electron micrograph of the exact same position. The radius of the spheres is $113 \mathrm{~nm}$. In both (A) and (B), the circle indicates the array of defects. The inset shows a detailed scanning electron micrograph of the feature (the scale bar equals $300 \mathrm{~nm}$ ). The array scatters light strongly and appears as a bright spot on the optical micrograph. The domain boundary, visible in both facets, confirms that the bright spot appears on the position of the array of defects. 


\subsection{Conclusions}

We have presented a method to realize both single material cavities, or point defects, and arrays of material cavities in individual colloids on the surface of silicon dioxide artificial opals by a focused ion beam milling technique. The relation between the milled defect size in individual colloidal particles and the milling parameters has been established. We show that the size of the defects can be controlled; in particular, defects much smaller than the size of a single sphere have been fabricated. Our results thus differ from previous studies where large area modifications have been written in polymeric opal films by electron beam [87] and by laser beams [88]. We conclude that focused ion beam milling allows for much smaller or more detailed cavities than these two techniques. Therefore, the cavities we fabricated can be used as size-selective chemical binders. We confirmed that milling not only occurs on the surface of the spheres, but into and through them as well. Therefore milled colloids can be used as building blocks of a lithographic mask.

Optical microscopy on our fabricated nano-cavities shows that these interact with light over a broad range of wave vectors. This results shows that our fabrication method to obtain milled defects in artificial opals is very interesting for potential optical cavities. To achieve an optical cavity inside a three dimensional photonic crystal we intend to cover a milled point defect, fabricated using the method described in this paper, with additional opal layers and subsequently invert the structure with either silicon or titanium dioxide (see C) and D) in Figure 6.2). 


\section{Reflectivity measured on fabricated structures}

\subsection{Introduction}

In many cases optical reflectivity experiments are among the first measurements performed to study the optical properties and the quality of newly developed photonic crystals, see for example references [63, 198, 209-211]. In particular, such measurements are a very suitable method to probe the band structure of these crystals [209]. Therefore, reflectivity spectra and band structure calculations are compared in order to judge the quality of fabricated photonic crystals, e.g., references [44, 58, 69, 70]. An additional advantage of optical reflectivity measurements is that the experimental setup does not require many components and is usually straightforward to assemble.

In this chapter we study the reflectivity of two-dimensional and threedimensional photonic crystals developed in this thesis. The measured reflectivity spectra are compared in detail to calculated band structures. Although all gaps in the band structure of a photonic crystal typically appear as peaks in the reflectivity spectrum, not all peaks are related to a gap as we will see in this chapter. We also describe the fabrication of three-dimensional crystals using focused ion beam milling.

\subsection{Optical setup}

Optical reflectivity experiments were performed with the setup shown in Figure 7.1. A beam of white light was generated by a broadband Fianium SC450-2 Supercontinuum white light source [212], which has a spectral range of $450-2500 \mathrm{~nm}$ and a $19 \mathrm{MHz}$ repetition rate with a 5 ps (master source) pulse width. A catoptric telescope, formed by two curved mirrors, increased 


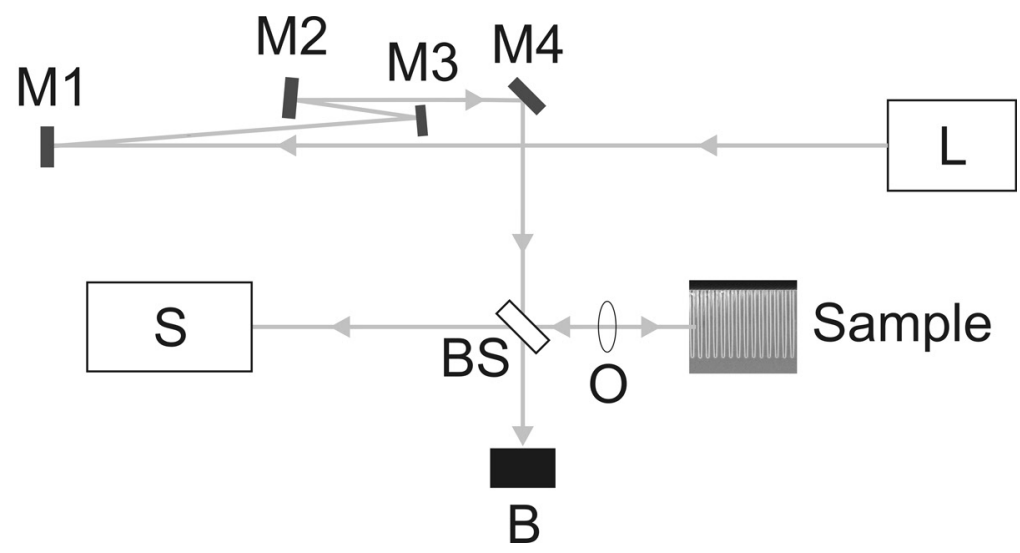

Figure 7.1: Schematic of the optical reflectivity setup. A broadband Fianium Supercontinuum white light laser $(L)$ was used as a source. M1 to M4 are mirrors. The beam diameter was increased by a catoptric telescope (curved mirrors M2 and M3) to completely fill the objective $(O)$. Via a pellicle beamsplitter (BS), the light was focused on the sample with an Ealing $74 \mathrm{x}$ reflecting objective (N.A. $=0.65$ ). The sample was mounted on a $x, y, z$ stage, equipped with an actuator to control the position of the sample with respect to the focus. Reflected light passed through the pellicle beamsplitter to the Biorad FT60 spectrometer $(S)$, which was equipped with an InGaAs long wavelength detector. Symbol (B) indicates a beamblock.

the beam diameter such that the aperture of the reflecting objective was completely filled. An aperture was placed in the incoming beam in order to select the central part of the beam which has the most uniform spectral distribution. The crystals were illuminated with light focused by a $74 \mathrm{x}$ Ealing reflecting objective with a numerical aperture of N.A. $=0.65$ [213]. This objective was chosen as it does not have chromatic abberation, which is ideal for broadband illumination. The intensity of the incident light was controlled by several neutral density filters. The samples were mounted on a x, y, z stage. The focus diameter was measured to be $6 \mu \mathrm{m}$ by scanning along a sharp edge. Reflected light passed through the pellicle beamsplitter to the Biorad FT60 Fourier transform spectrometer, which was equipped with an InGaAs long wavelength detector. For alignment we used the interferogram centerburst reading, which is the intensity integrated over all frequencies. An additional aperture was placed between the beamsplitter and the spectrometer to make sure that the amount of stray light reaching the detector was minimal. Reflectivity was calibrated by taking the ratio of the measured spectra to a reference spectrum, which was measured on a gold mirror using the same experimental conditions as the crystals. 
7.3. Optical reflectivity of two-dimensional photonic crystals

Table 7.1: Structural dimensions of both two-dimensional photonic crystals that were used for optical reflectivity measurements. The last row lists in which facet of Figure 4.16 the crystal is shown.

\begin{tabular}{|l|c|c|}
\hline & Crystal 1 & Crystal 2 \\
\hline Lattice parameter $a(\mathrm{~nm})$ & $691 \pm 15.0$ & $691 \pm 15.0$ \\
\hline Lattice parameter $c(\mathrm{~nm})$ & $486 \pm 10.6$ & $486 \pm 10.6$ \\
\hline Pore radius $R(\mathrm{~nm})$ & $153 \pm 12.3$ & $121 \pm 8.3$ \\
\hline Pore depth $h(\mu \mathrm{m})$ & $5.9 \pm 0.15$ & $5.8 \pm 0.13$ \\
\hline Tapering $\left(^{\circ}\right)$ & $0.30 \pm 0.10$ & $0.60 \pm 0.12$ \\
\hline Facet in Figure 4.16 & $\mathrm{B}$ & $\mathrm{C}$ \\
\hline
\end{tabular}

\subsection{Optical reflectivity of two-dimensional photonic crystals}

Optical reflectivity measurements were performed on two-dimensional photonic crystals fabricated using the Bosch reactive ion etching process, see chapter 4 . The two crystals crystal 1 and crystal 2 that were used are shown in Figure 4.16. The structural dimensions of these crystals are given in Table 7.1.

All reflectivity measurements are started by scanning the cross-section of the crystals in order to find areas that exhibit high reflectivity. Figure 7.2(A) shows the result of such a scan. The plot displays the interferogram intensity as a function of horizontal y-position on the crystal, i.e., from left to right ${ }^{1}$. The inset in (A) is an optical micrograph of the cross-section of crystal 1 after cleaving. By scanning the y-direction on the sample, areas that have high reflectivity are identified by peaks in the interferogram intensity. Areas with low reflectivity show up as troughs. By overlaying the optical micrograph (inset) with the interferogram, we have managed to make a correlation between low interferogram intensities and areas on the crystal that have undesired cleaving artifacts. These artifacts appear as a result of the cleaving of the sample to expose the cross-section of the crystals. In the inset in (A) the undesired cleaving artifacts are indicated by the black arrows and the white ellipsoid. Indeed, for all artifacts, troughs are visible in the intensity. In between these artifacts, the cross-section is homogeneous and the intensity is high. These regions have a high reflectivity and are regarded

${ }^{1}$ The interferogram intensity is not corrected for any background, nor is it normalized to the reference. 


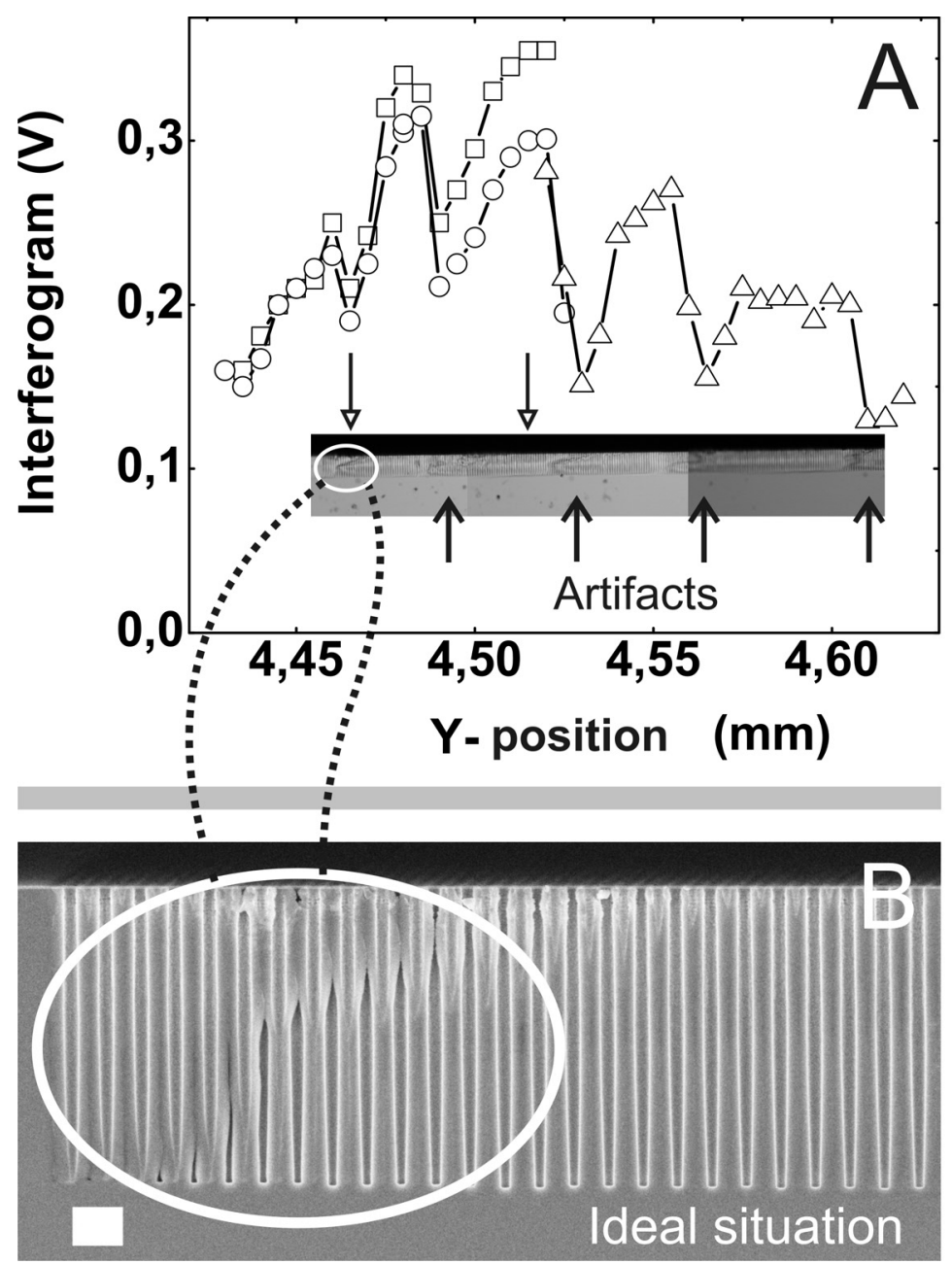

Figure 7.2: A) Reflected intensity versus y-horizontal position on crystal 1. The intensity is the interferogram value measured with the spectrometer. Three separate scans are shown. The inset is an optical micrograph on the same scale of the probed photonic crystal after cleaving. The black arrows and the white ellipsoid indicate undesired artifacts that appear due to cleaving. In between these artifacts are positions that show high reflectivity and hence are ideal for reflectivity. The open arrows indicate two positions of which reflectivity spectra are shown in Figure 7.3. B) Scanning electron micrograph of the left artifact shown in A). The artifact that scatters light is accented by the white ellipsoid. On the right side, the crystal surface is ideal for reflectivity. The scale bar equals $1 \mu \mathrm{m}$. 
7.3. Optical reflectivity of two-dimensional photonic crystals

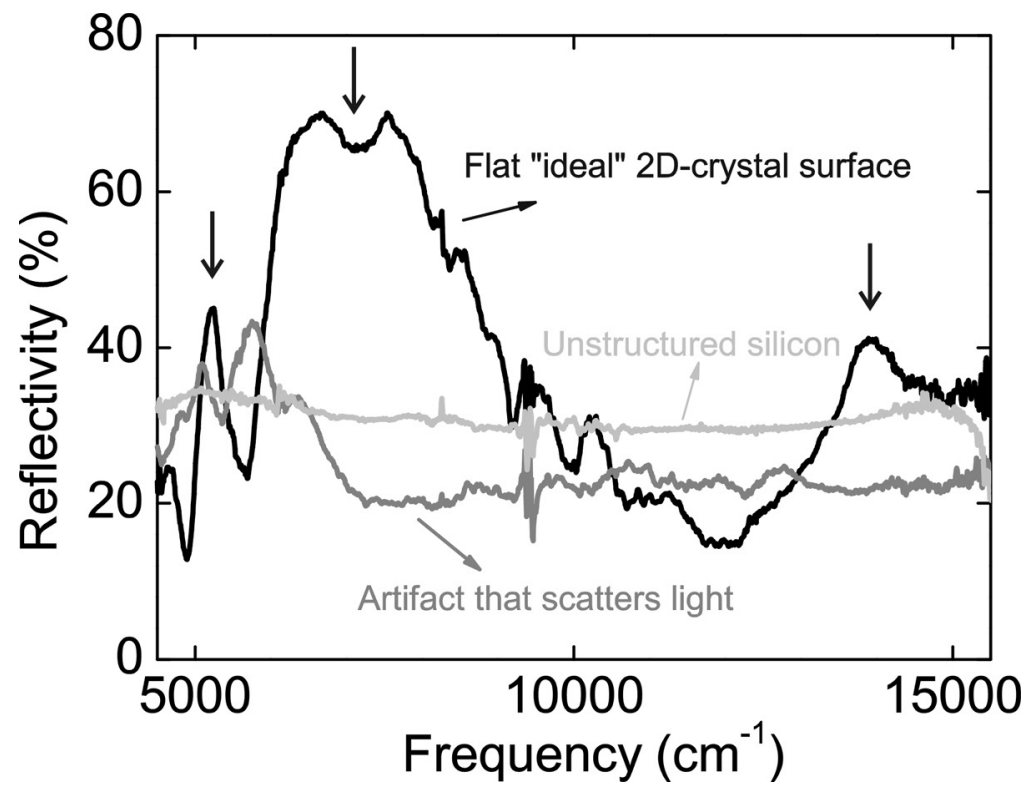

Figure 7.3: Reflectivity versus frequency measured in the $\Gamma$ - M' direction on crystal 1. Three spectra are shown: 1) Reflectivity spectrum on a flat, good quality, surfacearea (black curve). The reflectivity is high at more than $70 \%$. Three main peaks are visible around $\omega=5230,7160$, and $13960 \mathrm{~cm}^{-1}$ (vertical black arrows). The peak at $7160 \mathrm{~cm}^{-1}$ has a width of $40 \%$, representing the high photonic strength of the crystal. 2) Reflectivity of bulk silicon (light-grey). As expected the reflectivity is around 30\%. 3) Spectrum measured on an area with an undesired cleaving artifact that scatters light (grey). The reflectivity is far less than 30\%, which means that much light is scattered away from the detector. It is unclear why the set of peaks around $\omega=5740 \mathrm{~cm}^{-1}$ appears.

as ideal for optical reflectivity measurements.

Figure 7.2(B) shows a scanning electron micrograph of the left artifact shown in (A). The artifact consists of a terrace step, hence two layers of pores are visible. This is in contrast to the areas next to the artifact, where the cleaving is smooth and only one pore layer is visible. The terrace step scatters the incident light. As a result, less light is reflected towards the detector and a lower interferogram intensity is measured. This result clearly shows the importance of a smooth cross-section surface if one wants to do optical measurements on photonic crystals. Therefore, the surface polishing technique that was investigated in chapter 5 , section 5.3 is not only important for fabrication purposes, but is also relevant to prepare surfaces for optical measurements. 
Table 7.2: Frequencies $\omega$ of the three main reflectivity peaks of crystal 1 and crystal 2. Measured frequencies are determined with an accuracy of $\pm 10 \mathrm{~cm}^{-1}$. For comparison, the calculated $1^{\text {st }}$ order Bragg diffraction frequencies are given. The wavelengths $\lambda_{\text {Bragg }}$ of the Bragg peaks scale according to the ratio of the effective refractive indices $n_{\text {eff }}$, which are also given. For these calculations the error margins on the pore radii and the lattice parameters have not been taken into account. Note that peak 2 of crystal 1 appears as a result of $1^{\text {st }}$ order Bragg diffraction and a stop gap in the band structure.

\begin{tabular}{|l|c|c|c|}
\hline & Crystal 1 & Crystal 2 & Description \\
\hline Freq. Peak 1 $\left(\mathrm{cm}^{-1}\right)$ & 5230 & 5100 & Calculated stop gap \\
\hline Freq. Peak $2\left(\mathrm{~cm}^{-1}\right)$ & 7540 & 6730 & $1^{\text {st }}$ order Bragg \\
\hline Freq. Peak 3 $\left(\mathrm{cm}^{-1}\right)$ & 13960 & 12900 & $2^{\text {nd }}$ order Bragg \\
\hline Calculated Freq. $\left(\mathrm{cm}^{-1}\right)$ & 7604 & 6795 & $1^{\text {st }}$ order Bragg \\
\hline Calculated $n_{\text {eff }}$ & 2.71 & 3.03 & \\
\hline
\end{tabular}

Reflectivity versus frequency $\omega$ was measured for both crystal 1 and crystal 2. Figure 7.3 shows the results of measurements on the positions on crystal 1 indicated by the open arrows in Figure 7.2(A). Figure 7.5 shows the measured reflectivity on a highly reflecting area of crystal 2. Both reflectivity spectra show three characteristic peaks, indicated by the black arrows in both Figures. The frequencies of these peaks are compiled in Table 7.2.

The reflectivity spectrum that was measured on crystal 1 shows a surprisingly rich structure with three main peaks at $\omega=5230,7160$, and $13960 \mathrm{~cm}^{-1}$, see Figure 7.3. The measured reflectivity is high at more than $70 \%$, much higher than the $30 \%$ reflectivity of bulk silicon. This demonstrates that while the refractive index of the crystal is lower than of bulk silicon, the layers of pores are so well ordered that the Bragg interference yields a high reflectivity. The deviation of the reflectivity from ideal $100 \%$ is likely caused by the relatively large size of the focus compared to the pore depth of the structure. The reflectivity measured on an undesired cleaving artifact is almost as low as $20 \%$, which means that much light is scattered away from the detector instead of reflected. It is unclear why the set of three peaks around $\omega=5740 \mathrm{~cm}^{-1}$ appears. The crystal peak at $\omega=7160 \mathrm{~cm}^{-1}$ has a broad width of $40 \%$, representing the strong photonic interaction of the crystal with light.

A detailed assignment of the reflectivity features of crystal 1 is attempted in Figure 7.4. In the Figure the reflectivity spectrum is compared to a 


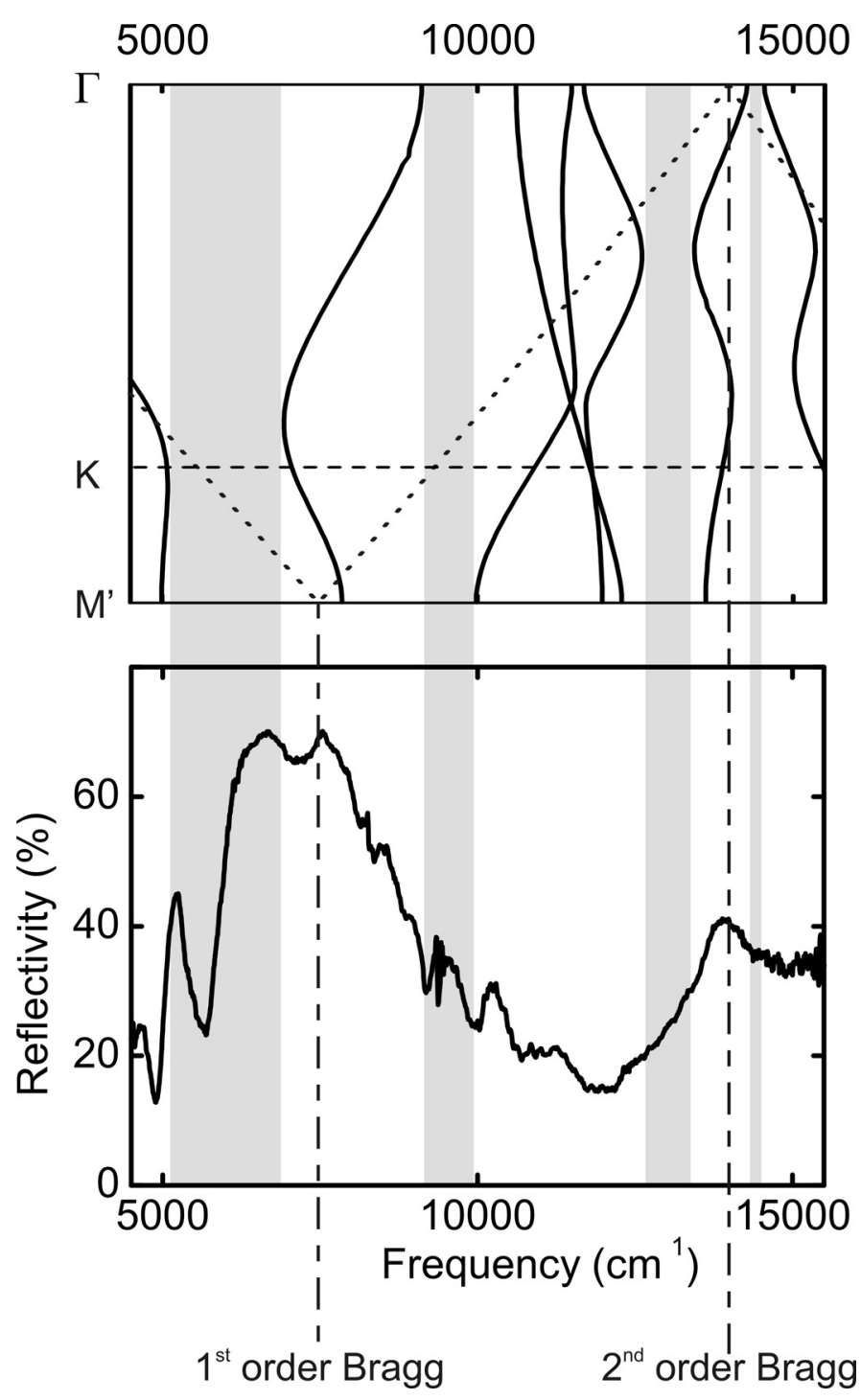

Figure 7.4: Reflectivity spectrum of crystal 1 compared to its calculated band structure in the $\Gamma-M$ ' direction in the crystal. The band structure was calculated for polarization perpendicular to the pore direction. The grey areas indicate stop gaps. The diagonal dotted lines are the light lines. The vertical dashed lines indicate the $1^{\text {st }}$ and $2^{\text {nd }}$ order Bragg conditions. Main peak positions are reported in Table 7.2. Peak 1 and the left side of peak 2 are explained by the first stop gap. The right side of peak 2 coincides with the $1^{\text {st }}$ order Bragg condition. The second stop gap appears at $\omega=9550 \mathrm{~cm}^{-1}$. The third and the fourth stop gaps appear in the range of the $2^{\text {nd }}$ order Bragg condition, which appears as peak 3. 
calculated band structure for the relevant $\Gamma-\mathrm{M}$ ' direction in the crystal ${ }^{2}$. The band structure features four stop gaps, indicated by the grey accented areas. In addition, the $1^{\text {st }}$ and $2^{\text {nd }}$ order Bragg reflections of the probed lattice planes of the crystal are indicated $[19,20]$, see the vertical dashed lines.

If we turn our attention to the high reflectivity peak in the spectrum at frequency $\omega=7160 \mathrm{~cm}^{-1}$ and compare this to the band structure, we see that it appears due to two different phenomena:

1) The low frequency side of the peak $\left(6680 \mathrm{~cm}^{-1}\right)$ originates from a stop gap in the band structure at the K-point.

2) The high frequency side of the peak $\left(7540 \mathrm{~cm}^{-1}\right)$ results from light that is $1^{\text {st }}$ order Bragg reflected. As a result there is a folded band between $\Gamma$ and $\mathrm{K}$ at frequencies between 7000 and $9000 \mathrm{~cm}^{-1}$. Since this band is far away from the light line, we surmise that it causes a high reflectivity.

Apparently, the peak at $\omega=7160 \mathrm{~cm}^{-1}$ is not a single peak, but the combination of a peak at $\omega=6680 \mathrm{~cm}^{-1}$ and a peak at $\omega=7540 \mathrm{~cm}^{-1}$. The small dip around $\omega=7130 \mathrm{~cm}^{-1}$ serves as a tell-tale signature of the fact that it is a double peak. Other conclusions based on the comparison between calculated band structure and measured reflectivity are the following:

a) The peak at $\omega=5230 \mathrm{~cm}^{-1}$ is (also) explained by the most left stop gap in the band structure calculation. It is unclear why the trough at $\omega=5180 \mathrm{~cm}^{-1}$ appears.

b) The second stop gap corresponds to a minor peak at $\omega=9550 \mathrm{~cm}^{-1}$.

c) The $2^{\text {nd }}$ order Bragg reflection at $\omega=13960 \mathrm{~cm}^{-1}$ corresponds to a high reflectivity peak of around $40 \%$. This peak does not have a corresponding gap in the band structure.

d) The third and fourth stop gaps do not show up as distinct peaks in the spectrum, which is most probably because the $2^{\text {nd }}$ order Bragg reflection has a much stronger effect.

A possible reason for the differences between the band structures and the experiments for frequencies $\omega>10000 \mathrm{~cm}^{-1}$ may be that at these frequencies silicon becomes absorbing. Hence the band structures should be interpreted with care.

\footnotetext{
${ }^{2}$ Calculated by A.P. Mosk using the MIT photonic bands package [127]. Corrected for silicon dispersion.
} 
7.3. Optical reflectivity of two-dimensional photonic crystals

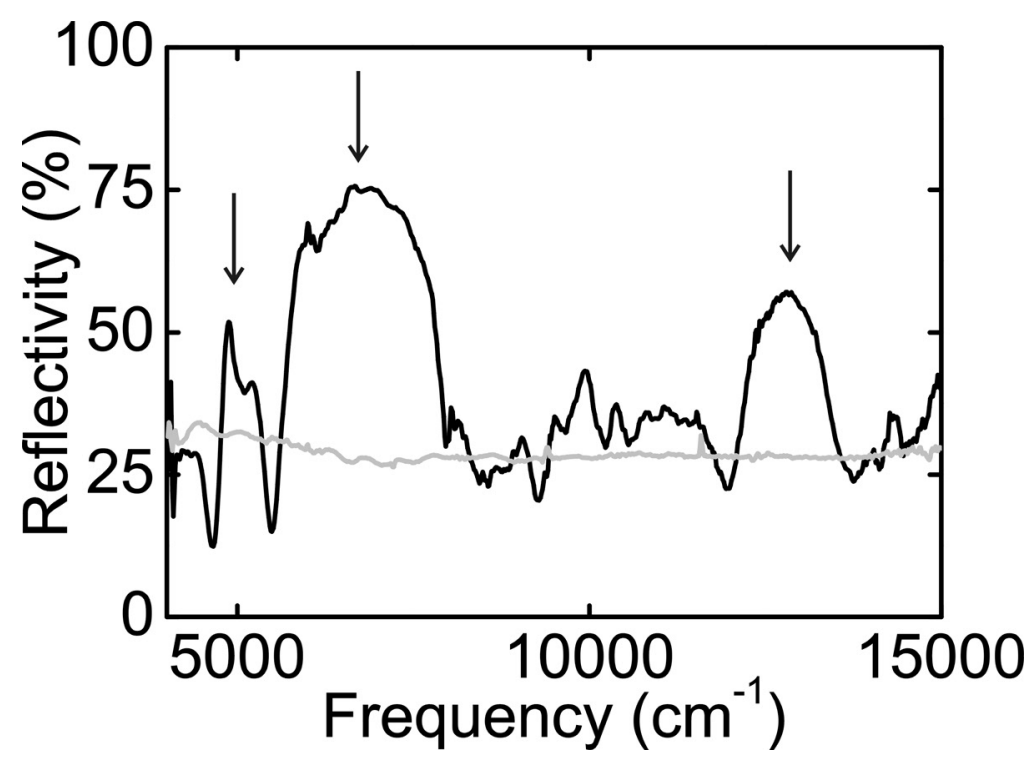

Figure 7.5: Reflectivity versus frequency measured in the $\Gamma$ - M' direction on crystal 2. The three characteristic peaks are indicated by the black arrows. The reflectivity of the $1^{\text {st }}$ order Bragg condition is high at more than $75 \%$ and the $2^{\text {nd }}$ order Bragg reflectivity is also high at more than $50 \%$, which indicate that the crystal quality is excellent. In light-grey the reflectivity of bulk silicon is shown. As expected the reflectivity is around $30 \%$.

Figure 7.5 shows measured normalized reflectivity versus frequency for crystal $2^{3}$. The spectrum is similar to the one reported in Figure 7.4, again with three main peaks. These peaks are centered around $\omega=5100,6730$, and $12900 \mathrm{~cm}^{-1}$. Reflectivity is as high as $75 \%$ for the dominant peak. The relative width of this peak is around $30 \%$, which shows that the photonic strength of this sample is also high. Similarly to Figure 7.4 the peak around $5100 \mathrm{~cm}^{-1}$ is explained by a stop gap in the band structure, the peak at $\omega=6730 \mathrm{~cm}^{-1}$ corresponds to $1^{\text {st }}$ order Bragg diffraction, and the peak at $\omega=12900 \mathrm{~cm}^{-1}$ to $2^{\text {nd }}$ order Bragg diffraction. The reflectivity of the $2^{\text {nd }}$ order Bragg diffraction is very high at $>50 \%$ (certainly in view of silicon absorption), which is an additional and compelling indication that crystal 2 has a high quality.

The frequencies of the Bragg reflections measured on crystal 2 are lower in frequency compared to crystal 1. This is explained as follows: due to the smaller radius $R$ of the pores of crystal 2 , the volume fraction of air $\phi_{\text {air }}$

\footnotetext{
${ }^{3}$ Reflectivity measured by A. Hartsuiker.
} 
in the crystal is lower. Consequently the effective refractive index $n_{e f f}$ is higher, which is described by:

$$
n_{e f f}=\sqrt{\left(\frac{\phi_{a i r}}{100} \cdot \varepsilon_{a i r}\right)+\left(\left(1-\frac{\phi_{a i r}}{100}\right) \cdot \varepsilon_{S i}\right)} .
$$

With increasing effective refractive index $n_{e f f}$, Bragg diffraction occurs at lower frequencies, as can be seen from Bragg's law modified for photonic crystals $[19,20]$ :

$$
\lambda_{\text {Bragg }}(\mathrm{nm})=\frac{10^{7}}{\omega_{\text {Bragg }}\left(\mathrm{cm}^{-1}\right)}=2 \cdot n_{\text {eff }} \cdot d(\mathrm{~nm}),
$$

with $d$ the probed lattice spacing. In our case $d=0.5 \cdot c$, with $c$ the lattice parameter. To summarize, Table 7.2 lists for both crystals the effective refractive indices $n_{\text {eff }}$ and the calculated $1^{\text {st }}$ order Bragg diffraction frequencies. The calculated and measured frequencies of the $1^{\text {st }}$ order Bragg diffraction peaks match within an accuracy of $<1 \%$. We also conclude that the frequencies of peaks 1 do not scale with the ratio of $n_{e f f}$, which was expected because these peaks do not occur due to Bragg diffraction.

These results show that our two-dimensional structures are high quality photonic crystals and that the structures are well-suited for optical experiments. It is apparent that our modified Bosch reactive ion etch process is compatible with optical experiments and nanophotonic applications. Furthermore we have shown that most of the features in the measured reflectivity spectrum can be explained by stop gaps in the band structure and by Bragg diffraction.

\subsection{Focused ion beam milling of prototype three dimensional photonic crystals}

In chapter 2 , section 2.3 , and in the introduction of chapter 5 , focused ion beam milling was introduced as a powerful method to fabricate prototype photonic crystals. In this section we briefly describe how focused ion beam milling was used to obtain three-dimensional crystals. For details, see Appendix A.

\subsubsection{Monoclinic photonic structures}

Three-dimensional photonic crystals were fabricated with two-dimensional samples as a starting point. The first pore set was obtained by reactive ion 
7.4. Focused ion beam milling of prototype three dimensional photonic crystals

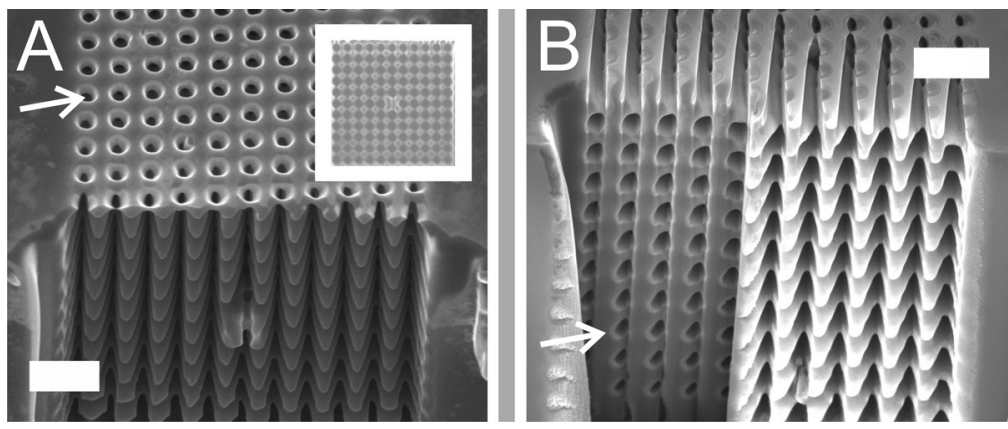

Figure 7.6: Scanning electron micrographs of two three-dimensional monoclinic $P$ photonic crystals, fabricated by i) reactive ion etching and ii) focused ion beam milling. The crystals are observed in a tilted fashion, with at the top the surface of the silicon wafer and below the cleaved cross-section. The scale bars equal $1 \mu \mathrm{m}$. A) The second set of pores was milled exactly through the first. The second pore set can be observed as dark windows penetrating the pore walls of the etched pores, see arrow. The crystal extends over many layers. One pore is not milled, to create a line-defect in the three-dimensional crystal. The inset shows a view parallel to the milled pores. The line defect is visible in the middle of the milled area. B) A crystal without line-defect. Part of the milled crystal was polished away to observe the inside. When going from the top-surface to deeper inside the crystal, the diameter of the pores is reduced due to redeposition of silicon. Redeposited silicon is visible as a lighter shade of grey on the edges of the milled pores, see arrow.

etching and subsequent focused ion beam milling yielded the second set of pores. The first pore set is shown in Figure 4.5(D). This array of pores has a centered rectangular lattice pattern. By milling additional sets of pores with a square pattern and a matching lattice parameters exactly through the first set of pores, three-dimensional monoclinic $P$ crystals are formed $[117]^{4}$, see Figure 7.6. Figure 7.6(A) shows that the second set of pores was indeed milled through the first set of pores, as intended. The milled pores are deep and pass through at least 11 layers of pores of the first set. Each crossing can be identified by a dark "window". The position of the windows shows that the pores were continuously milled exactly through the existing set of pores. In the center of the front-face of the crystal, one pore was intentionally not milled. This creates a line-defect in the three-dimensional crystal that may have interesting optical properties such as photonic band gap waveguiding. A detailed study of these optical properties is beyond the

${ }^{4}$ When taking into account deviations introduced by the fabrication processes, the new crystal is strictly speaking a triclinic system. 
scope of this thesis.

Figure 7.6(B) shows a crystal that was fabricated without line-defect. After milling, a few layers of the crystal were polished away to provide a view on the inside of the structure. When going along the milled pores from the top-surface to the inside of the structure, we observe that the diameter of the pores is reduced. This is due to redeposition of silicon, which is enhanced by the limited diffusion of the milled silicon to escape the structure at these deeper positions. A significant part of the silicon remains inside the crystal and redeposits on the walls of the pores. Redeposition appears to be absent in the top 5 or 6 layers of milled pores. Therefore the upper volume of this prototype crystal can be considered to be uniform.

The fabricated crystals are similar to crystals with a simple cubic lattice, which are reported to have calculated band gaps of more then $8.5 \%$ in the case of a volume fraction of air cylinders of $81 \%\left(\varepsilon_{S i}=13\right)$ [48]. The fabricated crystals are therefore expected to act as photonic crystals with moderate gaps. However, ultimately we performed these experiments as a proof-of-principle for the fabrication of inverse woodpile photonic crystals, see below.

\subsubsection{Inverse woodpile photonic crystal}

Using part of the two-dimensional crystal shown in Figure 7.2, a three dimensional inverse woodpile photonic crystal was obtained. Figure 7.7 shows the front-view (x-y plane) of the structure. The $\mathrm{x}-\mathrm{y}$ plane of this crystal consists of $9.5 \times 9.5$ unit cells. The surface radii of the milled pores appear to be uniform in size. Unfortunately the lattice parameters $a$ and $c$ and the pore radii $R$ of both pore sets are slightly different. Furthermore, the second pore set is displaced with respect to its ideal position by $143 \mathrm{~nm}$ on the left side and $113 \mathrm{~nm}$ on the right side of the crystal.

Taking into account the different radii of the two pore sets and the displacement of the second pore set, band structure calculations were performed for the middle of the $\mathrm{x}-\mathrm{y}$ plane of the $\mathrm{crystal}^{5}$. Figure 7.8 shows the resulting band structure. In all directions of the crystal one or more broad stop gaps are found. Due to the large stop gaps in the band structure, this crystal is a promising candidate for optical experiments as we show in section 7.5 where we probe the $\Gamma-\mathrm{Z}$ direction of this crystal. Unfortunately, due to

\footnotetext{
${ }^{5}$ Calculations were performed for a total of 17 (x, y) positions to represent the crystal. These calculations were evenly distributed over the $\mathrm{x}-\mathrm{y}$ plane of the crystal and considered the measured diameter of the etched pores at that $(\mathrm{x}, \mathrm{y})$ position, as well as the measured displacement. The result for the middle of the crystal was found to provide an adequate representation.
} 


\subsection{Reflectivity of a three-dimensional inverse woodpile photonic crystal}

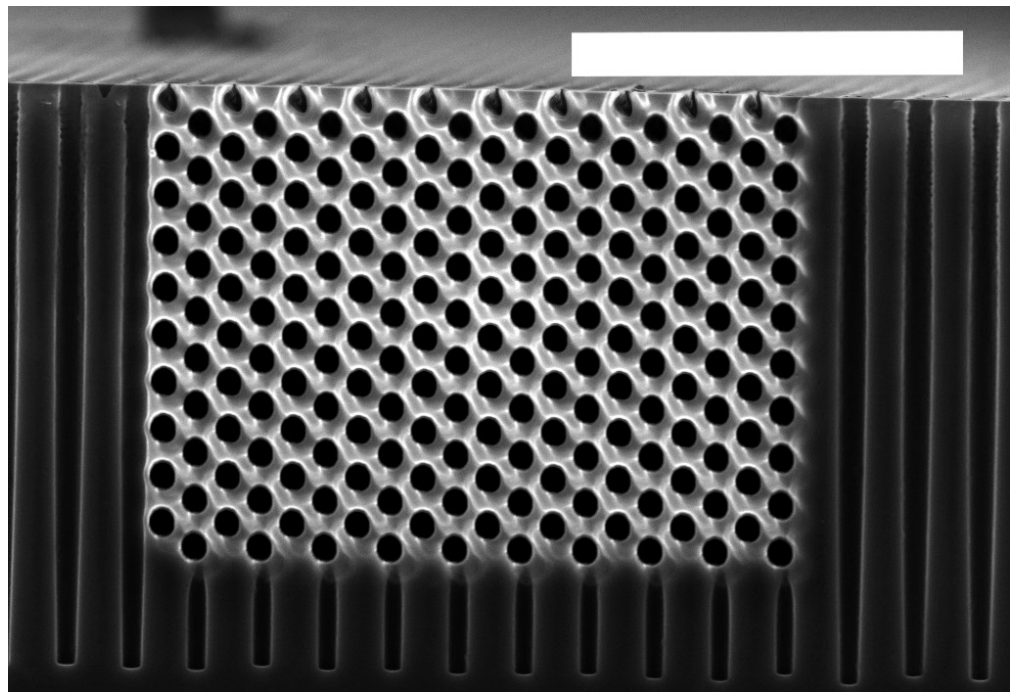

Figure 7.7: Scanning electron micrograph of the front-view of an inverse woodpile photonic crystal that was successfully fabricated by i) reactive ion etching (pores in-plane of the Figure) and ii) focused ion beam milling (pores perpendicular to the plane of the Figure). In this case some deviations from the perfect crystal geometry are present. Firstly, the lattice parameters $a$ and $c$ of both pore sets are slightly different, as well as the diameters of the two pore sets. Secondly, the mean diameter of the milled pores is estimated at $D_{\text {pore_FIB }}=270 \mathrm{~nm}$, whereas the measured mean diameter of the etched pores $D_{\text {pore } R I E}=306 \pm 24.5 \mathrm{~nm}$. Thirdly, the second pore set is misplaced from $113 \mathrm{~nm}$ up to $143 \mathrm{~nm}$ with respect to the first pore set. Finally, the radius of the etched pores varies slightly along the depth of the pores. The scalebar equals $4 \mu \mathrm{m}$.

the deviations from the ideal geometry, a band gap is not expected for this structure, as is illustrated by the grey horizontal bar which is interrupted. Nevertheless we see that this is a strongly interacting photonic crystal.

\subsection{Reflectivity of a three-dimensional inverse woodpile photonic crystal}

The optical reflectivity versus frequency was measured on the photonic crystal shown in Figure $7.7^{6}$. Reflectivity was measured with the optical axis parallel to the milled set of pores, i.e., parallel to the z-axis of the crystal. The measured reflectivity is as high as $68 \%$ for the dominant peak

\footnotetext{
${ }^{6}$ Reflectivity measured by A. Hartsuiker.
} 


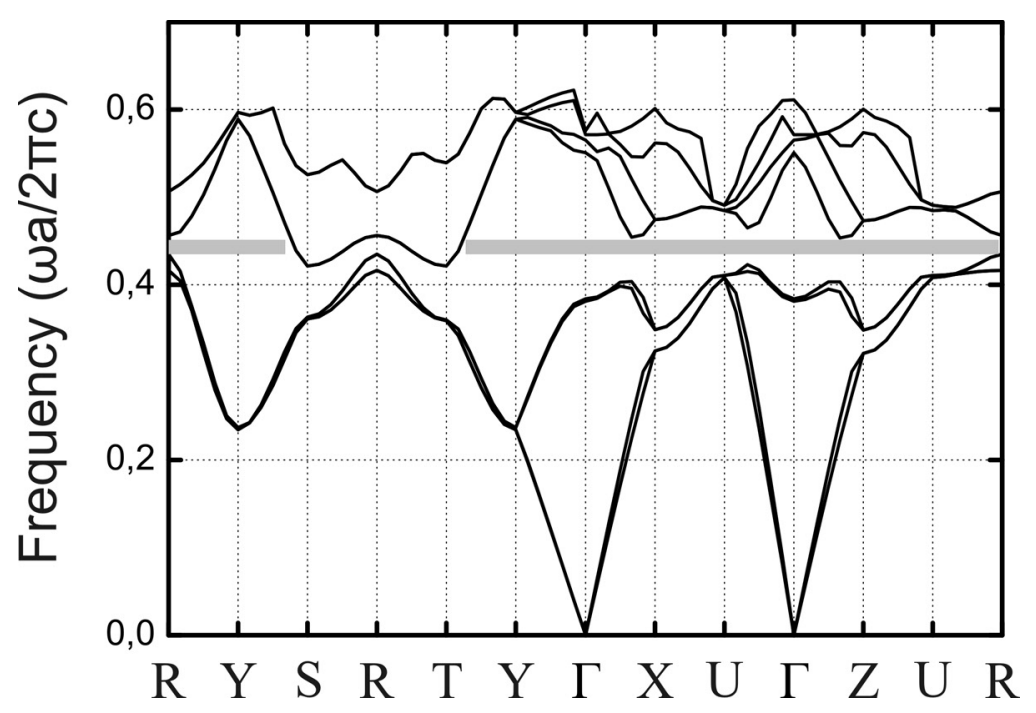

Figure 7.8: Calculated band structure for the crystal shown in Figure 7.7. This calculation takes into account the different radii of the two pore sets and the displacement of the second pore set with respect to the first. In all directions stop gaps are found. In particular in the $\Gamma-Z$ direction two large stop gaps are found. In contrast to the band structure of a perfect crystal, here the $\Gamma-X$ and $\Gamma-Z$ directions are no longer identical due to the lower symmetry of the crystal. Furthermore, the deviations from a perfect crystal cause the photonic band gap, typically visible between the $4^{\text {th }}$ and $5^{\text {th }}$ bands (grey horizontal bar), to be barely closed.

at frequency $\omega=5790 \mathrm{~cm}^{-1}$. The reflectivity spectrum contains four main peaks at $\omega=4950,5790,8560$, and $9250 \mathrm{~cm}^{-1}$. The peak at $\omega=5790 \mathrm{~cm}^{-1}$ has a distinct shoulder on its right side. The frequencies of the measured peaks are compiled in Table 7.3.

In Figure 7.9 the reflectivity spectrum is compared to a calculated band structure for the measured $\Gamma-\mathrm{Z}$ direction in the middle of the crystal. This band structure is taken from Figure 7.8. The band structure features two stop gaps, indicated by the grey accented areas. These two stop gaps appear in the reflectivity spectrum as peak 1 at $\omega=4950 \mathrm{~cm}^{-1}$ and as the shoulder of peak 2 at $\omega=5790 \mathrm{~cm}^{-1}$. Unfortunately, the band structure does not explain the occurrence of peak 2 itself and the origin of this peak remains unclear. Since our band structure calculation is currently limited to $\omega=8500 \mathrm{~cm}^{-1}$, peaks 3 and 4 cannot be compared to the band structure. However, peak 3 and peak 4 have frequencies in the approximate region where we expect the $1^{\text {st }}$ order Bragg reflection for this crystal. Further analysis shows that peak 3 


\section{Frequency ( $\omega \mathrm{a} / 2 \pi \mathrm{c})$}

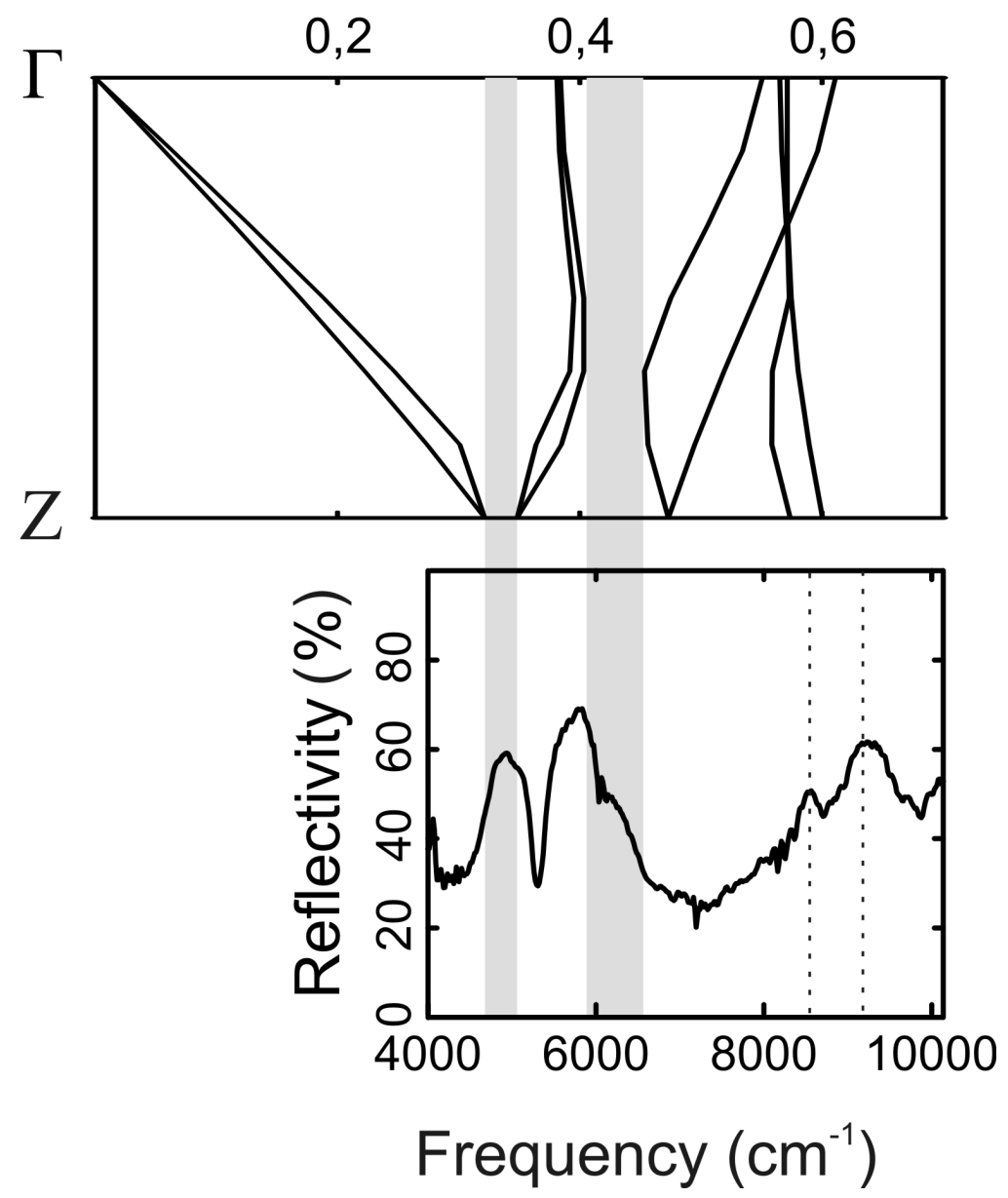

Figure 7.9: Reflectivity spectrum of the three-dimensional photonic crystal in Figure 7.7, compared to its calculated band structure in the $\Gamma-Z$ direction. Reflectivity is as high as $68 \%$. The grey areas indicate stop gaps. The vertical dashed lines indicate the calculated $1^{\text {st }}$ order Bragg frequencies for the positions on the crystal where the extreme volume fractions of silicon $\phi_{S i}$ are found (top left and lower right). The four main peak positions are reported in Table 7.3. Peak 1 and the shoulder of peak 2 correspond to the first and second stop gap. The left side of peak 2 remains unexplained. Peaks 3 and 4, and the area in-between these peaks, appear due to Bragg reflections. 
Table 7.3: Summarized frequencies $\omega$ of the four main reflectivity peaks of the three-dimensional photonic crystal. Measured frequencies are determined with an accuracy of $\pm 10 \mathrm{~cm}^{-1}$. The description column lists why the peak occurs.

\begin{tabular}{|c|c|c|}
\hline & $\omega\left(\mathrm{cm}^{-1}\right)$ & Description \\
\hline Peak 1 & 4950 & Stop gap in band structure \\
\hline Peak 2 & 5790 & unexplained, shoulder matches stop gap \\
\hline Peak 3 & 8560 & $1^{\text {st }}$ order Bragg, top left of crystal \\
\hline Peak 4 & 9250 & $1^{\text {st }}$ order Bragg, below middle right of crystal \\
\hline
\end{tabular}

at $\omega=8560 \mathrm{~cm}^{-1}$ corresponds to the Bragg diffraction expected for the top left of the crystal, where the volume fraction of silicon $\phi_{S i}$ is highest ${ }^{7}$. Peak 4 at $\omega=9250 \mathrm{~cm}^{-1}$ corresponds to the Bragg reflection expected just below the middle of the right side of the crystal, where the volume fraction of silicon $\phi_{S i}$ is lowest. On other positions on the crystal, Bragg reflectivity is expected to occur in-between these two extremes. Intuitively one would expect the Bragg reflection from the middle of the crystal to stand out. However, in this case it seems that the reflectivity of the two extreme frequencies appear as peaks. Further analysis is needed to obtain more insight in this surprising result. Nevertheless, we are confident that peaks 3 and 4 appear as the result of Bragg diffraction on the crystal.

We have shown that our prototype three-dimensional inverse woodpiles are high quality photonic crystals. As an example of the robustness of inverse woodpile crystals to geometrical deviations, high reflectivity is observed, even though there is a unintended misalignment of the second set of pores with respect to the first. Almost all peaks in the reflectivity spectrum are explained, either by stop gaps in the band structure or by Bragg diffraction. In addition, this result confirms focused ion beam milling as a powerful tool to fabricate prototype three-dimensional crystals.

\subsection{Conclusions}

Optical reflectivity experiments on our two-dimensional crystals show that these structures are strongly photonic. The fabricated inverse woodpile was shown to be strongly photonic as well, even though some deviations from the perfect geometry exist. This result supports our conclusion in chapter 2 that inverse woodpile photonic crystals are robust to fabrication induced

\footnotetext{
${ }^{7}$ Volume fraction determined on 17 , evenly distributed, positions on the crystal
} 
deviations. Mainly all features in the reflectivity spectra are explained by calculated stop gaps and Bragg diffraction.

In this chapter we have successfully demonstrated the fabrication of threedimensional inverse woodpile crystals using focused ion beam milling, including a structure with an intentional line defect. Band structure calculations for the fabricated inverse woodpile crystal indicates that for all directions stop gaps exist. Due to deviations from the perfect geometry, however, a photonic band gap is not present. Our results indicate that redeposition of silicon occurs, which reduces the uniform volume of the crystals. Nonetheless, the fabricated structures are clearly suitable for optical experiments.

The success of our optical experiments strongly supports focused ion beam milling as a powerful tool to prototype photonic crystals. It also serves as an additional incentive to fabricate these structures with the optimal fabrication techniques reported in this thesis, and hopefully obtain a crystal with a large photonic band gap. 
Table 7.4: Overview of settings used during focused ion beam milling of the three dimensional structures. The gallium ion beam of the workstation was accelerated to $30 \mathrm{kV}$.

\begin{tabular}{|l|c|c|}
\hline Setting & Monoclinic structures & Inverse woodpiles \\
\hline Beam current & $93 \mathrm{pA}$ & $9.7 \mathrm{pA}$ \\
\hline Mill duration & $1050 \mathrm{~s}$ & $5000 \mathrm{~s}$ \\
\hline
\end{tabular}

\subsection{Appendix A}

The first sets of pores were etched in the silicon wafer using the reactive ion etching process as described in chapter 4 . Subsequently, a FEI "xT Nova Nanolab 600" focused ion beam workstation was used to mill the pores in the 2D-crystal samples using a stream file. Where necessary the cross-section surface of the first set of pores was polished prior to milling the second set of pores. The gallium ion beam of the workstation was accelerated to $30 \mathrm{kV}$ and beam currents and milling times were set as shown in Table 7.4.

To image patterned samples, the scanning electron imaging functionality of the focused ion beam workstation was used. The high voltage was set at $5.00 \mathrm{kV}$ at a current of $98 \mathrm{pA}$. The working distance was typically set at $5 \mathrm{~mm}$ and the "through-the-lens" detector (TLD) was used to record the image. In other cases the structures were imaged by a LEO 1550 highresolution scanning electron microscope, with the high voltage set at $4 \mathrm{kV}$ and the working distance set around $2 \mathrm{~mm}$. 


\section{Summary and outlook}

The fabrication of three-dimensional inverse woodpile photonic crystals is highly desirable because of their predicted large photonic band gap, their conceptual ease of fabrication, and their robustness to withstand deviations from the ideal geometry that are intrinsic to nanofabrication. In this thesis, several fabrication methods were investigated to obtain three-dimensional inverse woodpile photonic crystals. Preferably, the entire process is compatible with methods used in the complementary metal oxide semiconductor (CMOS) industry to allow the incorporation of the photonic crystals in silicon integrated circuits. The effects of fabrication induced deviations on the photonic band gap of the structures were quantified. Furthermore, we have reported a new method to obtain material cavities that will act as optical cavities in opal photonic crystals.

Although the photonic band gap in inverse woodpile crystals is generally robust to disorder, it is sensitive to tapering of the pores. Nonetheless, within the limits of the fabrication methods, the fabricated crystals are expected to remain strongly photonic. In addition, a different crystal geometry was found that yields a slightly larger band gap. By changing the lithographic processes in our fabrication scheme, obtaining this crystal geometry is within reach.

An inverse woodpile photonic crystal can be obtained by etching two identical sets of pores aligned perpendicular to each other. The fabrication methods that were studied here are based on two approaches:

- We defined a preferred fabrication route to obtain photonic crystals with large volumes. This route comprises of photo electrochemical etching, Bosch-type reactive ion etching, and fabricating a holder wafer to facilitate accurate sample alignment.

- Rapid prototyping of photonic crystals using focused ion beam milling. 
The setup that was built for photo electrochemical etching was introduced and the best etching results to date were shown and analyzed in detail. In addition, the patterning processes was described which defined the etching positions. The etching results reported here define the direction to take in future work to obtain suitable sets of deep high quality pores for inverse woodpile photonic crystals:

- The etching setup was found to be suitable for etching, and some improvements are proposed to obtain our desired sets of pores.

- The experimental conditions with which these arrays of pores can be etched were determined and can be used during further investigations.

Periodic arrays of high aspect ratio nanopores were etched by Bosch-type reactive ion etching, as well as suitable arrays of pores for inverse woodpile photonic crystals. The pores were defined in a chromium mask by means of advanced deep UV scan and step technology. The parameters of the reactive ion etching process were carefully studied and the process was optimized for the formation of deep nanopores. Excellent quality pores with recordhigh aspect ratios up to 26 were realized. The etching rate was shown to be aspect ratio dependent, determined by the angular distribution of the etching ions. We have managed to derive an expression for the maximum obtainable aspect ratio and deduced that reducing the sidewall erosion is the preferred method to obtain high aspect ratio nanopores. Since our modified reactive ion etching process performs much better than expected, we see it as a novel route etch both sets of pores to realize inverse woodpile photonic crystals.

In the preferred fabrication route, a wafer holder is needed to accurately align the two sets of pores in an inverse woodpile crystal. In this thesis, the successful fabrication and testing of a suitable holder wafer is reported. Moreover, we report how samples were placed in such an holder wafer. We found that the alignment of the samples with the surface of the holder wafer is excellent and that the samples were solidly fixed to the holder wafer. Chromium was deposited on a wafer holder containing samples and a mask for etching was demonstrated using focused ion beam milling. A pre-requisite for good placement of samples in a holder wafer is that the cross-section surface of our 2D-crystal samples is smooth. We have demonstrated that samples containing nanopores can be polished using hardened photoresist for stabilization. In short: the developed holder wafer procedure is suitable for further processing of samples and can therefore be applied in the defined fabrication routes. 
We reported the fabrication of material nanocavities, and arrays thereof, in the surface of $f c c$-crystals of colloidal spheres by using a focused ion beam milling technique. Milling not only occurs on the surface of the spheres, but also through them, yielding what can be referred to as nano-beads. The radius and the position of the nanocavity can be controlled with nanometer precision and we determined the relation between defect size and milling time. Optical microscopy experiments show that the milled nano-sized point defects interact with light of many wave vectors, making our newly developed fabrication method very interesting to obtain optical cavities in (inverse) artificial opals. To do so, we plan to cover the milled point defects by additional opal layers and subsequently invert the obtained crystals with high-index materials such as silicon or titanium dioxide. The developed fabrication method is broadly applicable to focused ion beam milling on non-conducting substrates, which expands the possibilities of focused ion beam milling in general.

Prototype three-dimensional inverse woodpile crystals, were successfully fabricated using focused ion beam milling. Inherent to focused ion beam milling, the structures contain small amounts of redeposited silicon, which reduces the uniform volume of the crystals. The fabricated inverse woodpile deviates from the perfect geometry due to misalignment which barely prevents a photonic band gap from opening up. Nevertheless, band structure calculations indicate that broad stop gaps exist for all directions. Optical reflectivity experiments have confirmed that the fabricated inverse woodpile is indeed strongly photonic, which exemplifies the robustness of these crystals to withstand fabrication induced deviations. Finally, the success of these experiments confirm that focused ion beam milling is very useful to obtain prototype photonic crystals and to modify existing photonic structures.

Optical reflectivity experiments on two-dimensional structures obtained by reactive ion etching show that these are high quality photonic crystals. Most features in the reflectivity spectra of the studied two-dimensional crystals and of the three-dimensional inverse woodpile are quantitatively explained by calculated stop gaps and Bragg diffraction. It appears that complex reflectivity peaks arise, that are related to equally complex features in the band structures. Future work should include additional reflectivity measurements, i.e., along other crystal directions and by using light with well-defined polarization.

To summarize, the work contained in this thesis is a major step forward in our efforts to obtain inverse woodpile photonic crystals. The essential tools to do so, the fabrication methods, have been investigated. The reported optical experiments and the good quality of the fabricated prototype 
structure strongly motivate our confidence that soon the first true inverse woodpile photonic crystals with a large photonic band gap will be realized. Our results are an important guide for the last and final steps to be taken to reach that exciting goal. In addition, the first steps were reported of a process that results in high quality factor optical cavities in inverse artificial opals. 


\section{References}

[1] E. Karkoschka, The observer's sky atlas (Springer-Verlag, Berlin, 1990). - p.13.

[2] J. M. Enoch, History of mirrors dating back 8000 years, Optometry and vision science $\mathbf{8 3}, 775$ (2006). - p.13.

[3] G. Sines and Y. A. Sakellarakis, Lenses in antiquity, Am. J. Archaeology 91, 191 (1987). - p.13.

[4] H. N. Southworth, in Introduction to modern microscopy, edited by N. Mott and G. R. Noakes (Wykeham Publications (London) Ltd., London, 1975). - p.14.

[5] J. Muirden, Beginner's guide to astronomical telescope making (Pelham Books Ltd., London, 1975). - p.14.

[6] A. E. Siegman, in Lasers, edited by A. Kelly (University Science Books, Mill Valley, California, 1986). - p.14.

[7] G. R. Fowles, Introduction to modern optics, 2nd ed. (Dover Publications, Inc., New York, 1989). — p.14.

[8] T. H. Maiman, Stimulated optical radiation in Ruby, Nature 187, 493 (1960). - p.14.

[9] J. R. Vacca, Optical networking best practices handbook (WileyInterscience, Hoboken, New Jersey, 2007). - p.14.

[10] N. J. van der Veen, E. Rozniecka, L. A. Woldering, M. Chudy, J. Huskens, F. C. J. M. van Veggel, and D. N. Reinhoudt, Highly selective optical-sensing membranes, containing calix[4]arene chro- 
moionophores, for $\mathrm{Pb}^{2+}$ ions, Chem. Eur. J. 7, 4878 (2001). — p.14.

[11] B. C. Wilson and M. S. Patterson, The physics, biophysics and technology of photodynamic therapy, Phys. Med. Biol. 53, R61 (2008). - p.14.

[12] J. Usada, H. Kato, T. Okunaka, K. Furukawa, H. Tsutsui, K. Yamada, Y. Suga, H. Honda, Y. Nagatsuka, T. Ohira, M. Tsuboi, and T. Hirano, Photodynamic therapy (PDT) for lung cancers, J. Thorac. Oncol. 1, 489 (2006). - p.14.

[13] M. P. van Albada and A. Lagendijk, Observation of weak localization of light in a random medium, Phys. Rev. Lett. 55, 2692 (1985). — p.14.

[14] P. Wolf and G. Maret, Weak localization and coherent backscattering of photons in disordered media, Phys. Rev. Lett. 55, 2696 (1985). p.14.

[15] E. Yablonovitch, Inhibited spontaneous emission in solid-state physics and electronics, Phys. Rev. Lett. 58, 2059 (1987). — p.15, 19, 24, 26, 57, 89, and 145 .

[16] S. John, Strong localization of photons in certain disordered dielectric superlattices, Phys. Rev. Lett. 58, 2486 (1987). — p.15 and 19.

[17] K. J. Vahala, Optical microcavities, Nature 424, 839 (2003). — p.15, 25, 26, and 145 .

[18] J. D. Joannopoulos, R. D. Meade, and J. N. Winn, Photonic crystals molding the flow of light (Princeton University Press, Princeton, New Jersey, 1995). - p.15 and 16.

[19] W. L. Bragg, Diffraction of short electromagnetic waves by a crystal, Proc. Camb. Phil. Soc. 17, 43 (1913). — p.15, 172, and 174.

[20] W. L. Vos, R. Sprik, A. van Blaaderen, A. Imhof, A. Lagendijk, and G. H. Wegdam, Strong effects of photonic band structures on the diffraction of colloidal crystals, Phys. Rev. B 53, 16231 (1996). p.16, 172 , and 174 .

[21] A. F. Koenderink, Emission and transport of light in photonic crystals, Ph.D. thesis, University of Amsterdam, 2003. - p.17, 19, and 20.

[22] M. Megens, Structure and fluorescence of photonic colloidal crystals, Ph.D. thesis, University of Amsterdam, 1999. — p.18 and 148.

[23] H. M. van Driel and W. L. Vos, Multiple Bragg wave coupling in photonic band-gap crystals, Phys. Rev. B 62, 9872 (2000). - p.18.

[24] H. S. Sözüer, J. W. Haus, and R. Inguva, Photonic bands: convergence problems with the plane-wave method, Phys. Rev. B. 45, 13962 (1992). - p.18, 19, 20, and 21.

[25] J. V. Sanders, Colour of precious opal, Nature 204, 1151 (1964). p.19. 
[26] W. L. Vos, M. Megens, C. M. van Kats, and P. Bosëcke, X-ray diffraction of photonic colloidal single crystals, Langmuir 13, 6004 (1997). — p.19.

[27] P. Jiang, J. F. Bertone, K. Hwang, and V. Colvin, Single-crystal colloidal multilayers of controlled thickness, Chem. Mater. 11, 2132 (1999). - p.19, 148, and 150.

[28] D. J. Norris, E. G. Arlinghaus, L. Meng, R. Heiny, and L. E. Scriven, Opaline photonic crystals: how does self-assembly work?, Adv. Mater. 16, 1393 (2004). - p.19.

[29] Y. N. Xia, B. Gates, Y. D. Yin, and Y. Lu, Monodispersed colloidal spheres: old materials with new applications, Adv. Mater. 12, 693 (2000). - p.19.

[30] C. López, Materials aspects of photonic crystals, Adv. Mater. 15, 1679 (2003). - p.19 and 148.

[31] Y. Zhang, J. Wang, Y. Zhao, J. Zhai, L. Jiang, Y. Song, and D. Zhu, Photonic crystal concentrator for efficient output of dye-sensitized solar cells, J. Mater. Chem. 18, 2650 (2008). — p.19.

[32] S. V. Gaponenko, V. N. Bogomolov, E. P. Petrov, A. M. Kapitonov, D. A. Yarotsky, I. I. Kalosha, A. A. Eychmueller, A. L. Rogach, J. McGilp, U. Woggon, and F. Gindele, Spontaneous emission of dye molecules, semiconductor nanocrystals, and rare-earth ions in opalbased photonic crystals, J. Lightwave Technology 17, 2128 (1999). p.19.

[33] M. Megens, J. E. G. J. Wijnhoven, A. Lagendijk, and W. L. Vos, Light sources inside photonic crystals, J. Opt. Soc. Am. B 16, 1403 (1999). — p.19.

[34] P. Markowicz, C. Friend, Y. Shen, J. Swiatkiewicz, P. N. Prasad, O. Toader, S. John, and R. W. Boyd, Enhancement of two-photon emission in photonic crystals, Opt. Lett. 27, 351 (2002). - p.19.

[35] J. F. Galisteo-Lopez, F. López-Tejeira, S. Rubio, C. López, and J. Sánchez-Dehesa, Experimental evidence of polarization dependence in the optical response of opal-based photonic crystals, Appl. Phys. Lett. 82, 4068 (2003). - p.19.

[36] K. M. Ho, C. T. Chan, and C. M. Soukoulis, Existence of a photonic gap in periodic dielectric structures, Phys. Rev. Lett. 65, 3152 (1990).

- p.19 and 21.

[37] M. Campbell, D. N. Sharp, M. T. Harrison, R. G. Denning, and A. J. Turberfield, Fabrication of photonic crystals for the visible spectrum by holographic lithography, Nature 404, 53 (2000). — p.19.

[38] B. T. Holland, C. F. Blanford, and A. Stein, Synthesis of macrop- 
orous minerals with highly ordered three-dimensional arrays of spherical voids, Science 281, 538 (1998). — p.19.

[39] J. E. G. J. Wijnhoven and W. L. Vos, Preparation of photonic crystals made of air spheres in titania, Science 281, 802 (1998). — p.19 and 148 .

[40] A. A. Zakhidov, R. H. Baughman, Z. Iqbal, C. X. Cui, I. Khayrullin, S. O. Dantas, I. Marti, and V. G. Ralchenko, Carbon structures with three-dimensional periodicity at optical wavelengths, Science 282, 897 (1998). - p.19 and 148.

[41] P. V. Braun and P. Wiltzius, Electrochemically grown photonic crystals, Nature 402, 603 (1999). — p.19.

[42] K. M. Kulinowski, P. Jiang, H. Vaswani, and V. L. Colvin, Porous metals from colloidal templates, Adv. Mater. 12, 833 (2000). — p.19.

[43] A. Blanco, E. Chomski, S. Grabtchak, M. Ibisate, S. John, S. W. Leonard, C. Lopéz, F. Meseguer, H. Míguez, J. P. Mondia, G. A. Ozin, O. Toader, and H. M. van Driel, Large-scale synthesis of a silicon photonic crystal with a complete three-dimensional bandgap near 1.5 micrometres, Nature 405, 437 (2000). - p.20.

[44] Y. A. Vlasov, X. Z. Bo, J. C. Sturm, and D. J. Norris, On-chip natural assembly of silicon photonic band gap crystals, Nature 414, 289 (2001). - p.20, 27, and 165.

[45] E. Palacios-Lidón, A. Blanco, M. Ibisate, F. Meseguer, C. López, and J. Sánchez-Dehesa, Optical study of the full photonic band gap in silicon inverse opals, Appl. Phys. Lett. 81, 4925 (2002). — p.20.

[46] L. Bechger, Synthesis and Fluoresence of opal and air-sphere photonic crystals, Ph.D. thesis, University of Twente, 2003. - p.20 and 148.

[47] Z. Li and Z. Zhang, Fragility of photonic band gaps in inverse-opal photonic crystals, Phys. Rev. B 62, 1516 (2000). — p.20.

[48] H. S. Sözüer and J. W. Haus, Photonic bands: simple-cubic lattice, J. Opt. Soc. Am. B 10, 296 (1993). - p.20 and 176.

[49] R. Biswas, M. M. Sigalas, K. M. Ho, and S. Y. Lin, Three-dimensional photonic band gaps in modified simple cubic lattices, Phys. Rev. B 65, 205121 (2002). - p.20.

[50] M. Maldovan and E. L. Thomas, Photonic crystals: six connected dielectric networks with simple cubic symmetry, J. Opt. Soc. Am. B 22, 466 (2005). - p.20.

[51] S. Matthias, F. Müller, C. Jamois, R. B. Wehrspohn, and U. Gösele, Large-area three-dimensional structuring by electrochemical etching and lithography, Adv. Mater. 16, 2166 (2004). — p.20, 57, and 90.

[52] S. Y. Lin, J. G. Fleming, R. Lin, M. M. Sigalas, R. Biswas, and K. M. 
Ho, Complete three-dimensional photonic bandgap in a simple cubic structure, J. Opt. Soc. Am. B 18, 32 (2001). - p.20.

[53] S. Matthias, R. Hillebrand, F. Müller, and U. Gösele, Macroporous silicon: homogeneity investigations and fabrication tolerances of a simple cubic three-dimensional photonic crystal, J. Appl. Phys. 99, 113102 (2006). - p.20.

[54] M. Maldovan and E. L. Thomas, Diamond-structured photonic crystals, Nature Mat. 3, 593 (2004). - p.21.

[55] E. Yablonovitch, T. J. Gmitter, and K. M. Leung, Photonic band structure: the face-centered-cubic case employing nonspherical atoms, Phys. Rev. Lett. 67, 2295 (1991). — p.21.

[56] C. C. Cheng and A. Scherer, Fabrication of photonic band-gap crystals, J. Vac. Sci. Technol. B 13, 2696 (1995). — p.21.

[57] C. C. Cheng, V. Arbet-Engels, A. Scherer, and E. Yablonovitch, Nanofabricated three dimensional photonic crystals operating at optical wavelengths, Physica Scripta T68, 17 (1996). — p.21.

[58] A. Chelnokov, K. Wang, S. Rowson, P. Garoche, and J. M. Lourtioz, Near-infrared Yablonovite-like photonic crystals by focused-ion-beam etching of macroporous silicon, Appl. Phys. Lett. 77, 2943 (2000). p.21 and 165.

[59] K. Wang, A. Chelkonov, S. Rowson, and J. M. Lourtioz, Extremely high-aspect-ratio patterns in macroporous substrate by focused-ionbeam etching: the realization of three-dimensional lattices, Appl. Phys. A 76, 1013 (2003). - p.21.

[60] K. M. Ho, C. T. Chan, C. M. Soukoulis, R. Biswas, and M. Sigalas, Photonic band gaps in three dimensions: new layer-by-layer periodic structures, Solid State Comm. 89, 413 (1994). - p.21, 22, 24, 29, $30,37,38$, and 40 .

[61] S. Y. Lin, J. G. Fleming, D. L. Hetherington, B. K. Smith, R. Biswas, K. M. Ho, M. M. Sigalas, W. Zubzycki, S. R. Kurtz, and J. Bur, A three-dimensional photonic crystal operating at infrared wavelengths, Nature 394, 251 (1998). - p.21 and 91.

[62] J. G. Fleming and S. Y. Lin, Three-dimensional photonic crystal with a stop band from 1.35 to $1.95 \mu \mathrm{m}$, Optics Lett. 24, 49 (1999). — p.21.

[63] S. Noda, K. Tomoda, N. Yamamoto, and A. Chutinan, Full threedimensional photonic bandgap crystals at near-infrared wavelengths, Science 289, 604 (2000). - p.21 and 165.

[64] A. Chutinan and S. Noda, Spiral three-dimensional photonic band-gap structure, Phys. Rev. B 57, R2006 (1998). - p.21.

[65] O. Toader and S. John, Proposed square spiral microfabrication archi- 
tecture for large three-dimensional photonic band gap crystals, Science 292, 1133 (2001). - p.21.

[66] F. García-Santamaría, H. T. Miyazaki, A. Urquía, M. Ibisate, M. Belmonte, N. Shinya, F. Meseguer, and C. López, Nanorobotic manipulation of microspheres for on-chip diamond architectures, Adv. Mater. 14, 1144 (2002). - p.22 and 147.

[67] S. Takahashi, M. Okano, M. Imada, and S. Noda, Three-dimensional photonic crystals based on double-angled etching and wafer-fusion techniques, Appl. Phys. Lett. 89, 1231061 (2006). — p.22, 34, 91, and 129.

[68] R. Hillebrand, S. Senz, W. Hergert, and U. Gösele, Macroporous silicon-based three-dimensional photonic crystal with a large complete band gap, J. Appl. Phys. 94, 2758 (2003). — p.22, 24, 29, 30, 37, 38, 40, and 49.

[69] J. Schilling, J. White, A. Scherer, G. Stupian, R. Hillebrand, and U. Gösele, Three-dimensional macroporous silicon photonic crystal with large photonic band gap, Appl. Phys. Lett. 86, 011101 (2005). p.22, 129, and 165 .

[70] M. Hermatschweiler, A. Ledermann, G. A. Ozin, M. Wegener, and G. von Freymann, Fabrication of silicon inverse woodpile photonic crystals, Adv. Funct. Mater. 17, 2273 (2007). — p.22, 24, 52, and 165.

[71] H. B. Sun, S. Matsuo, and H. Misawa, Three-dimensional photonic crystal structures achieved with two-photon-absorption photopolymerization of resin, Appl. Phys. Lett. 74, 786 (1999). — p.22.

[72] E. Yablonovitch, T. J. Gmitter, R. D. Meade, A. Rappe, K. D. Brommer, and J. D. Joannopoulos, Donor and acceptor modes in photonic band structure, Phys. Rev. Lett. 67, 3380 (1991). - p.24 and 25.

[73] P. R. Villeneuve, S. H. Fan, and J. D. Joannopoulos, Microcavities in photonic crystals: mode symmetry, tunability, and coupling efficiency, Phys. Rev. B. 54, 7837 (1996). - p.24, 145, and 146.

[74] J. Vučković, M. Lončar, H. Mabuchi, and A. Scherer, Design of photonic crystal microcavities for cavity QED, Phys. Rev. E 65, 016608 (2001). - p.24.

[75] W. L. Vos, private communication, 2003. - p.24.

[76] P. V. Braun, S. A. Rinne, and F. Garćia-Santamaría, Introducing defects in 3D photonic crystals: state of the art, Adv. Mater. 18, 2665 (2006). - p.25.

[77] Q. Yan, L. Wang, and X. S. Zhao, Artificial defect engineering in threedimensional colloidal photonic crystals, Adv. Funct. Mater. 17, 3695 (2007). - p.25.

[78] E. Krioukov, D. J. W. Klunder, A. Driessen, J. Greve, and C. Otto, 
Sensor based on an integrated optical microcavity, Optics Lett. 27, 512 (2002). - p.25.

[79] G. Rempe, One atom in an optical cavity: spatial resolution beyond the standard diffraction limit, Appl. Phys. B 60, 233 (1995). — p.25.

[80] C. D. Bain and G. M. Whitesides, Molecular-level control over surface order in self-assembled monolayer films of thiols on gold, Science 240, 62 (1988). - p.25 and 145.

[81] G. B. Sigal, C. Bamdad, A. Barberis, J. Strominger, and G. M. Whitesides, A self-assembled monolayer for the binding and study of histidine tagged proteins by surface plasmon resonance, Anal. Chem. 68, 490 (1996). - p.25 and 145.

[82] A. Yariv, Y. Xu, R. K. Lee, and A. Scherer, Coupled-resonator optical waveguide: a proposal and analysis, Optics Lett. 24, 711 (1999). p.25.

[83] V. Yannopapas, A. Modinos, and N. Stefanou, Waveguides of defect chains in photonic crystals, Phys. Rev. B 65, 235201 (2002). — p.25.

[84] V. Lousse and S. Fan, Waveguides in inverted opal photonic crystals, Opt. Express 14, 866 (2006). — p.25.

[85] S. Y. Lin, J. G. Fleming, M. M. Sigalas, R. Biswas, and K. M. Ho, Photonic band-gap microcavities in three dimensions, Phys. Rev. B. 59, R15579 (1999). - p.25 and 146.

[86] S. P. Ogawa, M. Imada, S. Yoshimoto, M. Okano, and S. Noda, Control of light emission by 3D photonic crystals, Science 305, 227 (2004). p.25 and 146.

[87] P. Ferrand, J. Seekamp, M. Egen, R. Zentel, S. G. Romanov, and C. M. S. Torres, Direct electron-beam lithography on opal films for deterministic defect fabrication in three-dimensional photonic crystals, Microelectron. Eng. 73-74, 362 (2004). — p.25 and 164.

[88] W. M. Lee, S. A. Pruzinsky, and P. V. Braun, Multi-photon polymerization of waveguide structures within three-dimensional photonic crystals, Adv. Mater. 14, 271 (2002). - p.25 and 164.

[89] S. A. Rinne, F. García-Santamaría, and P. V. Braun, Embedded cavities and waveguides in three-dimensional silicon photonic crystals, Nature Photonics 2, 52 (2008). - p.25.

[90] L. Tang and T. Yoshie, Ultra-high Q three-dimensional photonic crystal nano-resonators, Opt. Express 15, 17254 (2007). — p.25.

[91] F. De Martini and G. R. Jacobovitz, Anomalous spontaneousstimulated-decay phase transition and zero-threshold laser action in a microscopic cavity, Phys. Rev. Lett. 60, 1711 (1988). — p.26.

[92] Y. Yamamoto and R. E. Slusher, Optical processes in microcavities, 
Physics Today 46, 66 (1993). - p.26.

[93] G. van Soest and A. Lagendijk, $\beta$ factor in a random laser, Phys. Rev. E 65, 047601 (2002). - p.26.

[94] H. Matsubara, S. Yoshimoto, H. Saito, Y. Jianglin, Y. Tanaka, and S. Noda, GaN photonic-crystal surface-emitting laster at blue-violet wavelengths, Science 319, 445 (2008). - p.26.

[95] O. Painter, A. Husain, A. Scherer, P. T. Lee, I. Kim, J. D. O'Brien, and P. D. Dapkus, Lithographic tuning of a two-dimensional photonic crystal laser array, IEEE Photonics Technology Letters 12, 1126 (2000). - p.26.

[96] J. D. Joannopoulos, P. R. Villeneuve, and S. Fan, Photonic crystals: putting a new twist on light, Nature 386, 143 (1997). - p.26 and 27.

[97] S. Fan, P. R. Villeneuve, J. D. Joannopoulos, and E. F. Schubert, High extraction efficiency of spontaneous emission from slabs of photonic crystals, Phys. Rev. Lett. 78, 3294 (1997). - p.26.

[98] M. Boroditsky, T. F. Krauss, R. Coccioli, R. Vrijen, R. Bhat, and E. Yablonovitch, Light extraction from optically pumped light-emitting diode by thin-slab photonic crystals, Appl. Phys. Lett. 75, 1036 (1999). - p.26.

[99] J. J. Wierer, M. R. Krames, J. E. Epler, N. F. Gardner, M. G. Craford, J. R. Wendt, J. A. Simmons, and M. M. Sigalas, In GaN/GaN quantum-well heterostructure light-emitting diodes employing photonic crystal structures, Appl. Phys. Lett. 84, 3885 (2004). — p.26.

[100] W. N. Ng, C. H. Leung, P. T. Lai, and H. W. Choi, Photonic crystal light-emitting diodes fabricated by microsphere lithography, Nanotechnology 19, 255302 (2008). — p.26.

[101] J. C. Knight, J. Broeng, T. A. Birks, and P. S. J. Russell, Photonic band gap guidance in optical fibers, Science 282, 1476 (1998). — p.27.

[102] P. Halevi, A. A. Krokhin, and J. Arriaga, Photonic crystals as optical components, Appl. Phys. Lett. 75, 2725 (1999). — p.27.

[103] S. Y. Lin, E. Chow, V. Hietala, P. R. Villeneuve, and J. D. Joannopoulos, Experimental demonstration of guiding and bending of electromagnetic waves in a photonic crystal, Science 282, 274 (1998). — p.27.

[104] M. Lončar, T. Doll, J. Vučković, and A. Scherer, Design and fabrication of silicon photonic crystal optical waveguides, J. Lightwave Technology 18, 1402 (2000). - p.27.

[105] Photonic crystals - advances in design, fabrication, and characterization, edited by K. Busch, S. Lölkes, R. B. Wehrspohn, and H. Föll (Wiley-VCH, Weinheim, 2004). — p.27.

[106] S. Smolka, M. Barth, and O. Benson, Selectively coated photonic crystal 
fiber for highly sensitive fluorescence detection, Appl. Phys. Lett. 90, 111101 (2007). - p.27.

[107] M. R. Lee and P. M. Fauchet, Nanoscale microcavity sensor for single particle detection, Optics Lett. 32, 3284 (2007). - p.27.

[108] P. Lodahl, A. F. van Driel, I. S. Nikolaev, A. Irman, K. Overgaag, D. Vanmaekelbergh, and W. L. Vos, Controlling the dynamics of spontaneous emission from quantum dots by photonic crystals, Nature 430, 654 (2004). - p.27.

[109] M. Aloshyna, S. Sivakumar, M. Venkataramanan, A. G. Brolo, and F. C. J. M. van Veggel, Significant suppression of spontaneous emission in $\mathrm{SiO}_{2}$ photonic crystals made with $\mathrm{Tb}^{3+}$ doped $\mathrm{LaF}_{3}$ nanoparticles, J. Phys. Chem. C 111, 4047 (2007). — p.27.

[110] S. Noda, M. Fujita, and T. Asano, Spontaneous-emission control by photonic crystals and nanocavities, Nature Photonics 1, 449 (2007). - p.27.

[111] C. Blum, A. P. Mosk, I. S. Nikolaev, V. Subramaniam, and W. L. Vos, Color control of natural fluorescent proteins by photonic crystals, Small 4, 492 (2008). - p.27.

[112] M. Grätzel, Photoelectrochemical cells, Nature 414, 338 (2001). p.27.

[113] P. M. Johnson, A. F. Koenderink, and W. L. Vos, Ultrafast switching of photonic density of states in photonic crystals, Phys. Rev. B 66, 081102 (2002). - p.27.

[114] S. W. Leonard, H. M. van Driel, J. Schilling, and R. B. Wehrspohn, Ultrafast band-edge tuning of a two-dimensional silicon photonic crystal via free-carrier injection, Phys. Rev. B. 66, 161102 (2002). — p.27.

[115] T. G. Euser, Ultrafast optical switching of photonic crystals, Ph.D. thesis, University of Twente, 2007. - p.27.

[116] A. Arsenault, S. Fournier-Bidoz, B. Hatton, H. Míguez, N. Tétrault, E. Vekris, S. Wong, S. Ming Yang, V. Kitaev, and G. A. Ozin, Towards the synthetic all-optical computer: science fiction of reality?, J. Mater. Chem. 14, 781 (2004). - p.27.

[117] C. Kittel, Introduction to Solid State Physics (Wiley, London, 1967), pp. 14-17. - p.30, 68, 94, and 175.

[118] A. N. Broers, A. C. F. Hoole, and J. M. Ryan, Electron beam lithography - Resolution limits, Microelectronic Eng. 32, 131 (1996). — p.31 and 90.

[119] L. Prodan, T. G. Euser, H. A. G. M. van Wolferen, C. Bostan, R. M. de Ridder, R. Beigang, K. J. Boller, and L. Kuipers, Large-area twodimensional silicon photonic crystals for infrared light fabricated with 
laser interference lithography, Nanotechnology 15, 639 (2004). — p.31 and 90 .

[120] V. Lehmann and H. Föll, Formation mechanism and properties of electrochemically etched trenches in n-type silicon, J. Electrochem. Soc. 137, 653 (1990). - p.31, 34, 59, 61, 83, and 84 .

[121] J. E. A. M. van den Meerakker, R. J. G. Elfrink, F. Roozeboom, and J. F. C. M. Verhoeven, Etching of deep macropores in 6in. Si wafers, J. Electrochem. Soc. 147, 2757 (2000). - p.31, 34, 57, 83, and 84.

[122] A. Birner, R. B. Wehrspohn, U. M. Gösele, and K. Busch, Silicon-based photonic crystals, Adv. Mater. 13, 377 (2001). - p.31 and 34.

[123] K. E. Bean, Anisotropic etching of silicon, IEEE Transactions of Electron Devices 25, 1185 (1978). - p.34 and 73.

[124] R. Kassing and I. W. Rangelow, Etching processes for high aspect ratio micro systems technology (HARMST), Microsyst. Technol. 3, 20 (1996). - p.34 and 93.

[125] F. Marty, L. Rousseau, B. Saadany, B. Mercier, O. Français, Y. Mita, and T. Bourouina, Advanced etching of silicon based on deep reactive ion etching for silicon high aspect ratio microstructures and threedimensional micro- and nanostructures, Microelectronics Journal 36, 673 (2005). - p.34 and 90.

[126] F. Laermer and A. Schilp, Method of anisotropically etching silicon, U.S. Patent 5501893 (1996). - p.35, 90, and 93.

[127] S. G. Johnson and J. D. Joannopoulos, Block-iterative frequencydomain methods for Maxwell's equations in a planewave basis, Opt. Express 8, 173 (2001). - p.37 and 172.

[128] J. Schilling and A. Scherer, 3D photonic crystals based on macroporous silicon: towards a large complete photonic bandgap, Photonics and Nanostructures 3, 90 (2005). - p.41 and 45.

[129] A. Chutinan and S. Noda, Effects of structural fluctuations on the photonic bandgap during fabrication of a photonic crystal, J. Opt. Soc. Am. B 16, 240 (1999). — p.45.

[130] R. J. Spry and D. J. Kosan, Theoretical analysis of the crystalline colloidal array filter, Appl. Spectroscopy 40, 782 (1986). — p.47.

[131] R. Hillebrand and W. Hergert, Scaling properties of a tetragonal photonic crystal design having a large complete bandgap, Photonics and Nanostructures 2, 33 (2004). - p.53.

[132] V. Lehmann, W. Hönlein, H. Reisinger, A. Spitzer, H. Wendt, and J. Willer, A novel capacitor technology based on porous silicon, Thin Solid Films 276, 138 (1996). — p.57.

[133] L. Pancheri, C. J. Oton, Z. Gaburro, G. Soncini, and L. Pavesi, Very 
sensitive porous silicon $\mathrm{NO}_{2}$ sensor, Sensors and Actuators B 89, 237 (2003). - p.57.

[134] R. W. Tjerkstra, J. G. E. Gardeniers, J. J. Kelly, and A. van den Berg, Multi-walled microchannels: Free-standing porous silicon membranes for use in $\mu T A S$, J. Microelecromech. Sys. 9, 495 (2000). — p.57.

[135] V. Lehmann, Electrochemistry of silicon (Wiley-VCH Verlag GmbH, Weinheim, 2002). - p.57, 58, 86, and 88.

[136] H. Föll, M. Christophersen, J. Carstensen, and G. Hasse, Formation and application of porous silicon, Mater. Sci. Eng. R 39, 93 (2002). — p.57,60, 83, and 87.

[137] U. Grüning, V. Lehmann, S. Ottow, and K. Busch, Macroporous silicon with a complete two-dimensional photonic band gap centered at $5 \mu \mathrm{m}$, Appl. Phys. Lett. 68, 747 (1996). — p.57.

[138] S. Rowson, A. Chelnokov, and J. M. Lourtioz, Macroporous silicon photonic crystals at $1.55 \mu \mathrm{m}$, Electronics Letters 35, 753 (1999). p.57.

[139] J. Schilling, R. B. Wehrspohn, A. Birner, F. Müller, R. Hillebrand, U. Gösele, S. W. Leonard, J. P. Mondia, F. Genereux, H. M. van Driel, P. Kramer, V. Sandoghar, and K. Busch, A model system for twodimensional and three-dimensional photonic crystals: macroporous silicon, J. Opt. A: Pure Appl. Opt. 3, S121 (2001). — p.57.

[140] V. Lehmann, The physics of macropore formation in low doped n-type silicon, J. Electrochem. Soc. 140, 2836 (1993). — p.59, 60, and 84.

[141] Photonic band gap materials, edited by C. M. Soukoulis (Kluwer Academic Publishers, Dordrecht, 1996). - p.59.

[142] J. Schilling, F. Müller, S. Matthias, R. B. Wehrspohn, and U. Gösele, Three-dimensional photonic crystals based on macroporous silicon with modulated pore diameter, Appl. Phys. Lett. 78, 1180 (2001). — p.61.

[143] M. Garín, T. Trifonov, A. Rodríguez, and R. Alcubilla, Infrared thermal emission in macroporous silicon three-dimensional photonic crystals, Appl. Phys. Lett. 91, 181901 (2007). — p.61.

[144] Properties of silicon (Institution of electrical engineers, London, 1988). — p.61.

[145] V. Lehmann and U. Grüning, The limits of macropore array fabrication, Thin Solid Films 297, 13 (1997). - p.62 and 83.

[146] J. E. A. M. van den Meerakker and M. R. L. Mellier, Kinetic and diffusional aspects of the dissolution of Si in HF solutions, J. Electrochem. Soc. 148, G166 (2001). - p.62.

[147] R. W. Tjerkstra, Isotropic etching of silicon in fluoride containing solutions as a tool for micromachining, Ph.D. thesis, University of 
Twente, 1999. - p.62.

[148] K. R. Williams and R. S. Muller, Etch rates for micromachining processing, J. Microelectromech. Syst. 5, 256 (1996). — p.66.

[149] M. H. A. Rifai, M. Christophersen, S. Ottow, J. Carstensen, and H. Föll, Dependence of macropore formation in $n$-Si on potential, temperature, and doping, J. Electrochem. Soc. 147, 627 (2000). — p.83.

[150] S. David, A. Chelnokov, and J. M. Lourtioz, Lateral confinement in macroporous silicon photonic crystal waveguides, J. Opt. A: Pure Appl. Opt. 4, 468 (2002). - p.84.

[151] R. B. Wehrspohn and J. Schilling, A model system for photonic crystals: macroporous silicon, Phys. Stat. Sol. A 197, 673 (2003). — p.84.

[152] Y. J. Lee and P. V. Braun, Tunable inverse opal hydrogel pH sensors, Adv. Mater. 15, 563 (2003). — p.89.

[153] E. Martines, K. Seunarine, H. Morgan, N. Gadegaard, C. D. W. Wilkinson, and M. O. Riehle, Superhydrophobicity and superhydrophilicity of regular nanopatterns, Nanoletters 5, 2097 (2005). p.89.

[154] F. Roozeboom, A. L. A. M. Kemmeren, J. F. C. Verhoeven, F. C. van den Heuvel, J. Klootwijk, H. Kretschman, T. Frič, E. C. E. van Grunsven, S. Bardy, C. Bunel, D. Chevrie, F. LeCornec, S. Ledain, F. Murray, and P. Philippe, Passive and heterogeneous integration towards a Si-based System-in-Package concept, Thin Solid Films 504, 391 (2006). - p.89 and 90.

[155] W. Bogaerts, P. Bienstman, and R. Baets, Scattering at sidewall roughness in photonic crystal slabs, Opt. Lett. 28, 689 (2003). — p.89.

[156] D. Gerace and L. C. Andreani, Effects of disorder on propagation losses and cavity Q-factors in photonic crystal slabs, Photonics and Nanostructures 3, 120 (2005). - p.89.

[157] R. Ferrini, R. Houdré, H. Benisty, M. Qiu, and J. Moosburger, Radiation losses in planar photonic crystals: two-dimensional representation of hole depth and shape by an imaginary dielectric constant, J. Opt. Soc. Am. B 20, 469 (2003). - p.89.

[158] A. Chelnokov, S. David, K. Wang, F. Marty, and J. M. Lourtioz, Fabrication of 2-D and 3-D silicon photonic crystals by deep etching, IEEE Journal of Selected Topics in Quantum Electronics 8, 919 (2002). - p.89, 90, and 91 .

[159] N. Tétreault, G. von Freymann, M. Deubel, M. Hermatschweiler, F. Pérez-Willard, S. John, M. Wegener, and G. A. Ozin, New route to three-dimensional photonic bandgap materials: silicon double inversion of polymer templates, Adv. Mater. 18, 457 (2006). — p.90. 
[160] M. P. Rao, M. F. Aimi, and N. C. MacDonald, Single-mask, threedimensional microfabrication of high-aspect-ratio structures in bulk silicon using reactive ion etching lag and sacrificial oxidation, Appl. Phys. Lett. 85, 6281 (2004). - p.90, 102, and 113.

[161] C. Chang, Y. F. Wang, Y. Kanamori, J. J. Shi, Y. Kawai, C. K. Lee, K. C. Wu, and M. Esashi, Etching submicrometer trenches by using the Bosch process and its applications to the fabrication of antireflection structures, J. Micromech. Microeng. 15, 580 (2005). — p.90 and 99.

[162] M. Zhou, X. Chen, Y. Zeng, J. Xu, and W. Lu, Fabrication of twodimensional infrared photonic crystals by deep reactive ion etching on Si wafers and their optical properties, Solid State Communications 132, 503 (2004). - p.90.

[163] R. A. Gottscho, C. W. Jurgensen, and D. J. Vitkavage, Microscopic uniformity in plasma etching, J. Vac. Sci. Technol. B 10, 2133 (1992). - p.90, 102, 113, 117, and 118.

[164] W. Bogaerts, R. Baets, P. Dumon, V. Wiaux, S. Beckx, D. Taillaert, B. Luyssaert, J. van Campenhout, P. Bienstman, and D. van Thourhout, Nanophotonic waveguides in silicon-on-insulator fabricated with CMOS technology, J. Lightwave Technology 23, 401 (2005). - p.90 and 91.

[165] I. Märki, M. Salt, H. P. Herzig, R. Stanley, L. E. Melhaoui, P. Lyan, and J. Fedeli, Characterization of buried photonic crystal waveguides and microcavities fabricated by deep ultraviolet lithography, J. Appl. Phys. 98, 013103 (2005). - p.90.

[166] L. Fu, J. M. Miao, X. X. Li, and R. M. Lin, Study of deep silicon etching for micro-gyroscope fabrication, Appl. Surf. Sci. 177, 78 (2001). p.91.

[167] G. Villanueva, J. A. Plaza, A. Sánchez-Amorez, J. Bausells, E. Martínes, J. Samitier, and A. Errachid, Deep reactive ion etching and focused ion beam combination for nanotip fabrication, Materials Science and Engineering C 26, 164 (2006). - p.91 and 99.

[168] C. Lee, D. B. Graves, and M. A. Lieberman, Role of etch products in polysilicon etching in a high-density chlorine discharge, Plasma Chemistry and Plasma Processing 16, 99 (1996). — p.91.

[169] K. R. Ryan and I. C. Plumb, A model for the etching of silicon in $\mathrm{SF}_{6} / \mathrm{O}_{2}$ plasmas, Plasma Chemistry and Plasma Processing 10, 207 (1990). - p.92.

[170] J. T. C. Lee, N. Layadi, K. V. Guinn, H. L. Maynard, F. P. Klemens, D. E. Ibbotson, I. Tepermeister, P. O. Egan, and R. A. Richardson, Comparison of advanced plasma sources for etching applications, J. 
Vac. Sci. Technol. B 14, 2510 (1996). - p.92.

[171] C. Cardinaud, M. C. Peignon, and P. Y. Tessier, Plasma etching: principles, mechanisms, application to micro- and nano-technologies, Appl. Surf. Sci. 164, 72 (2000). - p.93.

[172] S. Tachi, K. Tsujimoto, and S. Okudaira, Low-temperature reactive ion etching and microwave plasma etching of silicon, Appl. Phys. Lett. 52, 616 (1988). - p.93.

[173] J. W. Bartha, J. Greschner, M. Puech, and P. Maquin, Low temperature etching of $\mathrm{Si}$ in high density plasma using $\mathrm{SF}_{6} / \mathrm{O}_{2}$, Microelec. Eng. 27, 453 (1995). - p.93.

[174] K. R. Williams, K. Gupta, and M. Wasilik, Etch rates for micromachining processing - part II, J. of Microelectromech. Syst. 12, 761 (2003). - p.94.

[175] J. Kiihamäki, H. Kattelus, J. Karttunen, and S. Franssila, Depth and profile control in plasma etched MEMS structures, Sensors and Actuators 82, 234 (2000). - p.99 and 113.

[176] J. Yeom, Y. Wu, J. C. Selby, and M. A. Shannon, Maximum achievable aspect ratio in deep reactive ion etching of silicon due to aspect ratio dependent transport and the microloading effect, J. Vac. Sci. Technol. B 23, 2319 (2005). - p.102 and 116.

[177] M. J. de Boer, J. G. E. Gardeniers, H. V. Jansen, E. Smulders, M. J. Gilde, G. Roelofs, J. N. Sasserath, and M. Elwenspoek, Guidelines for etching silicon MEMS structures using fluorine high-density plasmas at cryogenic temperatures, J. Microelectromechanical Systems 11, 385 (2002). - p.104 and 108.

[178] C. W. Jurgensen, Sheath collision processes controlling the energy and directionality of surface bombardment in $\mathrm{O}_{2}$ reactive ion etching, J. Appl. Phys. 64, 590 (1988). — p.104.

[179] C. J. D. Craigie, T. Sheenan, V. N. Johnson, S. L. Burkett, A. J. Moll, and W. B. Knowlton, Polymer thickness effects on Bosch etch profiles, J. Vac. Sci. Technol. B 20, 2229 (2002). — p.104.

[180] C. B. Labelle, V. M. Donnelly, G. R. Bogart, R. L. Opila, and A. Kornblit, Investigation of fluorocarbon plasma deposition from $c-C_{4} F_{8}$ for use as passivation during deep silicon etching, J. Vac. Sci. Technol. A 22, 2500 (2004). - p.104.

[181] M. A. Blauw, T. Zijlstra, and E. van der Drift, Balancing the etching and passivation in time-multiplexed deep dry etching of silicon, J. Vac. Sci. Technol. B 19, 2930 (2001). — p.106 and 111.

[182] M. Boufnichel, S. Aachboun, F. Grangeon, P. Lefaucheux, and P. Ranson, Profile control of high aspect ratio trenches of silicon. I. Effect of 
process parameters on local bowing, J. Vac. Sci. Technol. B 20, 1508 (2002). - p.108.

[183] Y. M. Im, Y. B. Hahn, and S. J. Pearton, Level set approach to simulation of feature profile evolution in a high-density plasma-etching system, J. Vac. Sci. Technol. B 19, 701 (2001). — p.108.

[184] Z. K. A. Chou and K. Najafi, Fabrication of out-of-plane curved surfaces in Si by utilizing RIE lag, in Proceedings of the 15th IEEE International Conference on MEMS, Las Vegas, NV, January 20-24 145 (2002). - p.113.

[185] M. Boufnichel, P. Lefaucheux, S. Aachboun, R. Dussart, and P. Ranson, Origin, control and elimination of undercut in silicon deep plasma etching in the cryogenic process, Microelectronic Eng. 77, 327 (2005). - p.113.

[186] I. W. Rangelow, Critical tasks in high aspect ratio silicon dry etching for microelectromechanical systems, J. Vac. Sci. Technol. A 21, 1550 (2003). - p.116.

[187] H. V. Jansen, M. de Boer, and M. Elwenspoek, The black silicon method VI: high aspect ratio trench etching for MEMS applications, Proc. IEEE, The Ninth Ann. Int. Workshop Micro Elec. Mech. Syst. 250 (1996). - p.116.

[188] H. V. Jansen, M. de Boer, R. Wiegerink, N. Tas, E. Smulders, C. Neagu, and M. Elwenspoek, RIE lag in high aspect ratio trench etching of silicon, Microelectronic Eng. 35, 45 (1997). - p.117, 118, and 121.

[189] R. J. Davis, Image potentials and the dry etching of submicron trenches with low-energy ions, Appl. Phys. Lett. 59, 1717 (1991). — p.118.

[190] M. Elwenspoek and H. V. Jansen, Silicon micromachining (Camridge University Press, Cambridge, UK, 1998). — p.118 and 121.

[191] For information on reduced $\chi^{2}$ minimization the Reader is referred to any textbook on statistics in physics. Also the help function in Origin 7 gives an excellent summary. - p.120.

[192] K. Wang, A. Chelnokov, S. Rowson, P. Garoche, and J. M. Lourtioz, Focused-ion-beam etching in macroporous silicon to realize threedimensional photonic crystals, J. Phys. D: Appl. Phys. 33, L119 (2000). - p.129.

[193] R. W. Tjerkstra, F. B. Segerink, J. J. Kelly, and W. L. Vos, Fabrication of three-dimensional nanostructures by focused ion beam milling, J. Vac. Sci. Technol. B 26, 973 (2008). — p.129.

[194] http://www.southbaytech.com. — p.137.

[195] M. J. Cryan, M. Hill, D. Cortaberria Sanz, P. S. Ivanov, P. J. Heard, L. Tian, S. Yu, and J. M. Rorison, Focused ion beam-based fabrica- 
tion of nanostructured photonic devices, IEEE J. Sel. Topics Quantum Electron. 11, 1266 (2005). - p.141.

[196] F. Burmeister, C. Schäfle, T. Matthes, M. Böhmisch, J. Boneberg, and P. Leiderer, Colloid monolayers as versatile lithographic masks, Langmuir 13, 2983 (1997). — p.145.

[197] R. D. Pradhan, I. I. Tarhan, and G. H. Watson, Impurity modes in the optical stop bands of doped colloidal crystals, Phys. Rev. B. 54, 13721 (1996). - p.146.

[198] M. H. Qi, E. Lidorikis, P. T. Rakich, S. G. Johnson, J. D. Joannopoulos, E. P. Ippen, and H. I. Smith, A three-dimensional optical photonic crystal with designed point defects, Nature 429, 538 (2004). — p.146 and 165 .

[199] D. M. Schaefer, R. Reifenberger, A. Patil, and R. P. Andres, Fabrication of 2-dimensional arrays of nanometer-size clusters with the atomic-force microscope, Appl. Phys. Lett. 66, 1012 (1995). — p.147.

[200] S. Reyntjens and R. Puers, A review of focused ion beam applications in microsystem technology, J. Micromech. Microeng. 11, 287 (2001). - p.148 and 160 .

[201] D. J. Norris and Y. A. Vlasov, Chemical approaches to threedimensional semiconductor photonic crystals, Adv. Mater. 13, 371 (2001). - p.148.

[202] W. Stöber, A. Fink, and E. Bohn, Controlled growth of monodisperse silica spheres in the micron size range, J. Colloid Interface Sci. 26, 62 (1968). - p.148.

[203] F. J. Arriagada and K. Osseo-Assare, Synthesis of nanosize silica in aerosol ot reverse microemulsions, J. Colloid Interface Sci. 170, 8 (1995). - p.148.

[204] J. E. G. J. Wijnhoven, L. Bechger, and W. L. Vos, Fabrication and characterization of large macroporous photonic crystals in titania, Chem. Mater. 13, 4486 (2001). - p.148.

[205] R. J. Young, J. R. A. Cleaver, and H. Ahmed, Characteristics of gas-assisted focused ion-beam etching, J. Vac. Sci. Technol. B 11, 234 (1993). - p.149.

[206] M. Geissler and Y. N. Xia, Patterning: Principles and some new developments, Adv. Mater. 16, 1249 (2004). - p.160.

[207] C. F. Bohren and D. R. Huffman, Absorption and scattering of light by small particles (Wiley-Interscience, New York, 1983), pp. 107-111. - p.160.

[208] E. Flück, Local interaction of light with periodic photonic structures, Ph.D. thesis, University of Twente, 2003. - p.162. 
[209] M. S. Thijssen, R. Sprik, J. E. G. J. Wijnhoven, M. Megens, T. Narayanan, A. Lagendijk, and W. L. Vos, Inhibited light propagation and broadband reflection in photonic air-sphere crystals, Phys. Rev. Lett. 83, 2730 (1999). - p.165.

[210] E. Palacios-Lidón, J. F. Galisteo-López, B. H. Juárez, and C. López, Engineered planar defects embedded in opals, Adv. Mater. 16, 341 (2004). - p.165.

[211] R. V. Nair and R. Vijaya, Three-dimensionally ordered photonic crystal heterostructures with a double photonic stop band, J. Appl. Phys. 102, 056102 (2007). — p.165.

[212] http://www.fianium.com/. — p.165.

[213] http://64.143.63.33/pdf/Microscope_Components.pdf. — p.166. 



\section{Nederlandse samenvatting}

\section{Fotonische kristallen en trilholtes}

Door de mensheid zijn vele verschillende objecten en apparaten bedacht die te maken hebben met licht. Gedreven door de wens om de propagatie en het maken van licht te controleren zijn bijvoorbeeld spiegels, gloeilampen, lenzen en lasers gemaakt. In 1987 zijn structuren beschreven, fotonische kristallen, die het ultieme gereedschap zijn om licht op te sluiten in "kleine doosjes". Hiermee kunnen bijvoorbeeld hele kleine en energie-efficiënte lasers gemaakt worden.

Fotonische kristallen zijn periodieke structuren waarin twee verschillende materialen elkaar op een geordende manier afwisselen. Deze beide materialen hebben verschillende brekingsindices voor licht ${ }^{1}$. De periodiciteit van de twee materialen, meestal een halfgeleider ${ }^{2}$ en lucht, heeft een lengteschaal in de orde van de golflengte van licht. Door de bijzondere manier waarop de fotonische kristallen zijn opgebouwd treedt een verschijnsel op dat Bragg reflectie heet. Bragg reflectie berust op het verstrooien van licht aan de roostervlakken in het fotonische kristal. Voor bepaalde golflengten resulteert dit in bepaalde richtingen in constructieve interferentie van licht. Licht met deze

\footnotetext{
${ }^{1}$ De brekingsindex van een materiaal is een maat voor de verhouding van de snelheid van licht in dat materiaal tot de snelheid van licht in vacuüm.

${ }^{2}$ Een halfgeleider is een materiaal met een geleidbaarheid voor elektronen dat ligt tussen de geleidbaarheid van isolerende materialen en geleidende metalen. Deze materialen, met name silicium, zijn de basis voor de chip-industrie.
} 
golflengten, of liever gezegd, frequenties, kan in het fotonisch kristal niet in deze richtingen voortbewegen en wordt door het kristal gereflecteerd. Dit is schematisch weergegeven in Figuur 1(A). Het frequentiebereik waarvoor propagatie van licht is verboden noemen we stopgap. In reflectiviteitsmetingen resulteert de stopgap in een piek. Bij hoge gemeten reflecties en brede pieken is er sprake van een sterke interactie van het fotonische kristal met licht, en dat is wat men wil bereiken met deze strukturen.

Naarmate het verschil in brekingsindex van de beide materialen waarvan het fotonische kristal is gemaakt toeneemt, wordt ook de interactie van het kristal met licht sterker. Hierdoor worden de stopgaps breder. Uiteindelijk kan de situatie ontstaan dat propagatie van licht in geen enkele richting meer mogelijk is. Het frequentiebereik waarvoor dit geldt noemen we de fotonische bandkloof. Het onstaan van een bandkloof is een bijzonder verschijnsel, want het is totaal anders dan alledaagse verschijnselen die we kennen van bijvoorbeeld de kleurselectie in een glas aarbeienranja door absorptie (opname) van al het licht dat niet rood is. De fotonische bandkloof is de basis voor verschillende mogelijke toepassingen van fotonische kristallen.

Licht met frequenties in de fotonische bandkloof is verboden in het kristal. Hierdoor is spontane emissie door lichtbronnen in het kristal niet toegestaan: het licht kan niet door de bron worden uitgezonden. Het normale gedrag van de lichtbronnen is dus verstoord. Dit verschijnsel is interessant omdat hiermee bijvoorbeeld zonnecellen en LED's efficiënter gemaakt kunnen worden.

Een bijzondere situatie ontstaat wanneer in de kern van een fotonisch kristal een kleine holte geïntroduceerd wordt. In Figuur 1(B) is een dergelijke holte schematisch weergeven in het midden van een fotonisch kristal. Door deze holte is er lokaal geen fotonische bandkloof aanwezig. Hierdoor kan een lichtbron in de nabijheid van deze holte wel een foton ${ }^{3}$ uitzenden, maar omdat de holte volledig is omringd door het fotonische kristal, kan het foton niet uit de holte ontsnappen en is hierin dus opgesloten. Een dergelijke holte is een unieke driedimensionale kooi voor licht. We noemen deze holte een trilholte. Deze kunnen gebruikt worden om nauwkeurige sensoren voor kleine deeltjes of (de al genoemde) zeer efficiënte lasers te maken.

\section{Verschillende soorten fotonische kristallen}

Een voorbeeld van een fotonische kristal is te zien in Figuur 2. Dit is een zogenaamd kunstmatig opaal en bestaat uit gestapelde polystyreen colloïdale deeltjes die omringd zijn door lucht. De zwarte balken duiden drie van de

\footnotetext{
${ }^{3}$ Fotonen zijn de elementaire energiedeeltjes waaruit licht bestaat.
} 


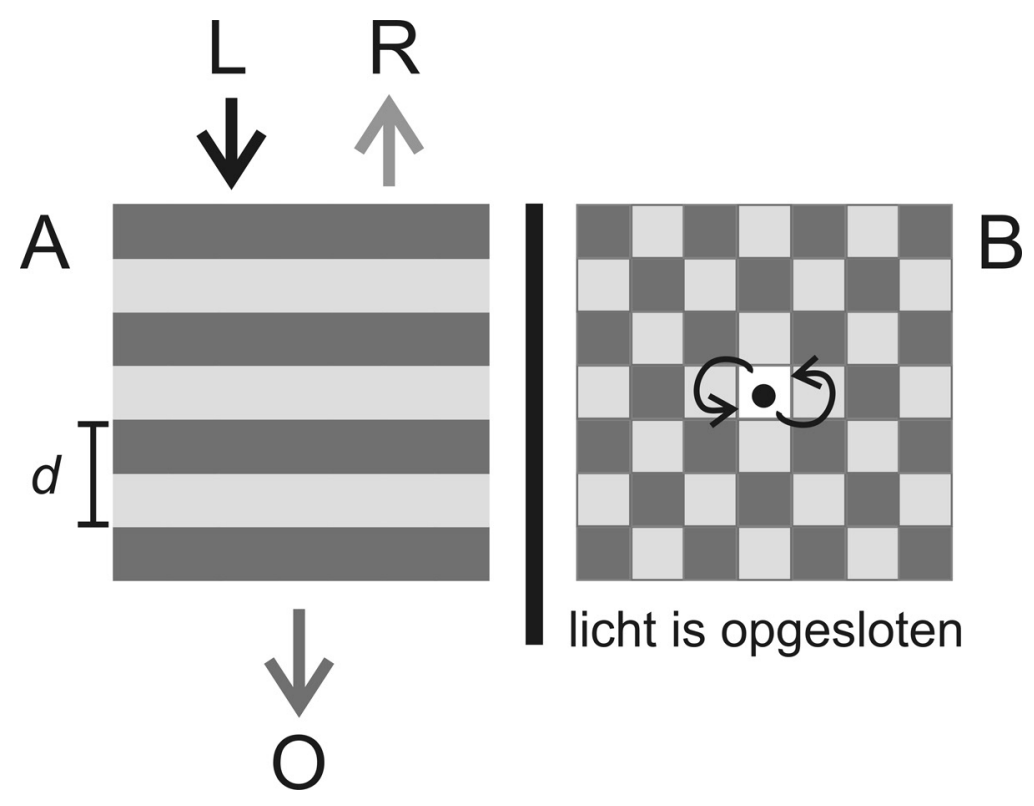

Figure 1: A) Schematische weergave van één set van roostervlakken in een fotonisch kristal opgebouwd uit twee materialen (licht en donker). Aan deze roostervlakken vindt Bragg-reflectie plaats waardoor een bepaalde kleur van het invallende licht $(L)$ gereflecteerd wordt $(R)$. De golflengte van het gereflecteerde licht is gelijk aan twee maal de afstand $d$. De overige kleuren $(O)$ gaan door het fotonische kristal. B) Schematische weergave van een trilholte in een fotonisch kristal. De lichtbron (zwarte punt) kan zijn foton uitzenden, maar omdat het omringd is door een kristal met een bandkloof kan het licht niet uit de trilholte ontsnappen: het licht zit in een driedimensionale kooi.

roostervlakrichtingen aan waar reflectie van licht kan optreden. In deze Figuur zien we voornamelijk de bovenste laag van het kristal, maar doordat er in het midden van dit kristal één bol ontbreekt is de daaronder liggende laag goed te zien. Vaak bestaat een dergelijk fotonisch kristal uit meerdere honderden lagen en is dus echt driedimensionaal met een groot volume.

Deze kristallen zijn te maken door een suspensie van polystyreen bollen te laten bezinken. De getoonde bolstapeling ontstaat dan vanzelf en is zeer geordend. Deze manier om fotonische kristallen te maken is zeer populair vanwege zijn relatief eenvoudige fabricage, maar helaas kan er in dit soort kristallen geen fotonische bandkloof ontstaan. Een interessanter kristal ontstaat door de structuur te "inverteren". Dit wil zeggen dat waar in Figuur 2 lucht zit, een materiaal met een hoge brekingsindex komt en andersom. De structuren die dan ontstaan noemen we luchtbolkristallen of inverse opalen. 


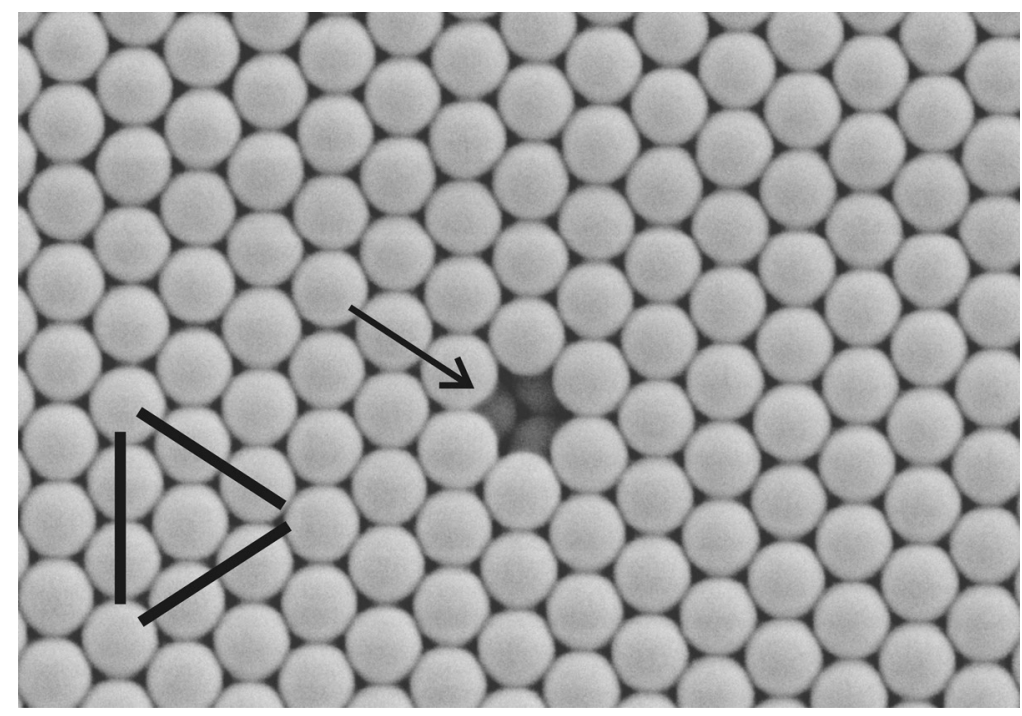

Figure 2: Voorbeeld van een kunstmatig opaal gemaakt van collö̈dale deeltjes. Deze zijn gestapeld in een geordend fcc rooster. De zwarte balken geven roostervlakrichtingen aan waar Bragg reflectie kan plaatsvinden. Op de positie van de ontbrekende bol (pijl) is te zien dat een dergelijk kristal uit meerdere lagen bestaat. Deze verschillende lagen zijn ook roostervlakken waaraan Bragg reflectie kan plaatsvinden. Vaak bestaan dergelijke kristallen uit meerdere honderden lagen.

Er is (theoretisch) voorspeld dat in luchtbolkristallen een fotonische bandkloof kan bestaan. In de praktijk blijkt echter dat dit vaak niet het geval is omdat deze bandkloof gevoelig is voor wanorde. Hierdoor wordt de bandkloof smaller of verdwijnt zelfs geheel als er kleine fouten in het kristal zitten, zoals bijvoorbeeld verschillen in bolgrootte, fouten in de positie van de luchtbollen, of fouten in de stapeling. Omdat deze fouten onvermijdelijk zijn, is het noodzakelijk andere soorten fotonische kristallen te fabriceren om de fotonische bandkloof te kunnen bestuderen.

Andere fotonische kristallen waarvoor een bandkloof is voorspeld zijn de zogenaamde houtstapel kristallen. Deze bestaan uit op elkaar gestapelde lagen van staafjes. Deze structuur lijkt op een stapel hout en ontleent daaraan zijn naam. De kristallen zijn echter niet gemaakt van hout, maar van halfgeleiders met een hoge brekingsindex zoals silicium. De voorspelde interactie van deze structuren met licht is sterk. Omdat deze kristallen laag voor laag worden opgebouwd ontstaat er wanorde in de structuur en is de volume van het te maken kristal beperkt. Hierdoor is het moeilijk de voorspelde sterke interactie met licht daadwerkelijk te benaderen. 


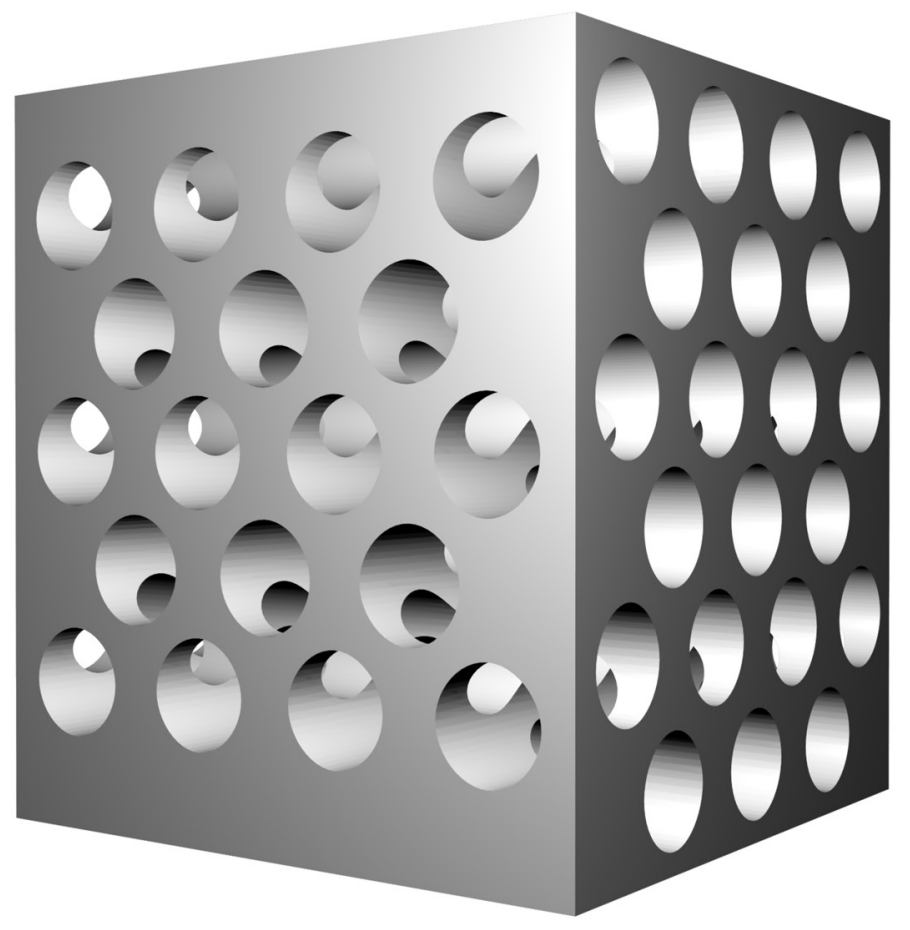

Figure 3: Illustratie van een geïnverteerde houtstapel fotonisch kristal. Dit kristal bestaat uit poriën van lucht in silicium. Goed is te zien hoe de twee verschillende patronen van poriën uitgelijnd zijn ten opzichte van elkaar. Ook is te zien hoe de verschillende poriën elkaar overlappen. Deze structuur is kubisch. De radius van de porien is typisch rond de $300 \mathrm{~nm}$ en hun onderlinge afstand is ongeveer $400 \mathrm{~nm}$.

\section{Geïnverteerde “houtstapel” kristallen}

Naast de bovengenoemde luchtbolkristallen en houtstapel kristallen bestaan er vele variaties van fotonische kristallen waarvoor een fotonische bandkloof is voorspeld. Bijzonder interessant is de fabricage van driedimensionale geïnverteerde houtstapel kristallen, want de voorspelde bandkloof is breder in een geïnverteerde houtstapel vergeleken met een "gewone" houtstapel. Uit berekeningen blijkt dat deze bandkloof robuust is tegen wanorde in de structuur, zoals afwijkende poriediameters en fouten in de positie van de poriën.

Een geïnverteerde houtstapel bestaat uit staafjes lucht "gestapeld" in 


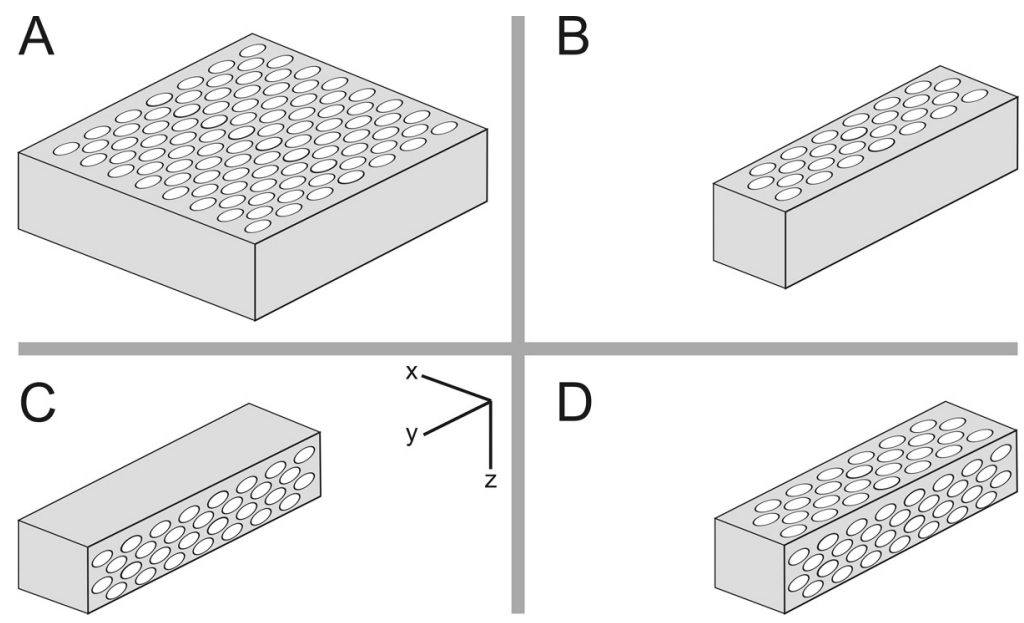

Figure 4: Fabricageschema voor het maken van een geïnverteerde houtstapel fotonisch kristal. A) Gestart wordt met het etsen van een eerste patroon van poriën van lucht in silicium. B) Een stuk wordt uit het gemaakte monster gezaagd. De gekozen afmetingen zijn dusdanig dat dit stuk in de houderwafer (zie hoofdstuk 5) past. C) Het uitgezaagde stuk wordt gekanteld en accuraat geplaatst in de houderwafer (niet zichtbaar). D) Na nauwkeurig uitgelijnd te zijn wordt het tweede patroon van poriën van lucht geëtst. Hierdoor ontstaat het gewenste geïnverteerde houtstapel kristal.

een hoog brekingsindex materiaal, zie Figuur 3. Twee identieke patronen van deze staafjes, poriën genaamd, staan loodrecht op elkaar en zijn zeer nauwkeurig op elkaar uitgelijnd. Het fabricageconcept bestaat uit slechts twee etsstappen ${ }^{4}$ waarmee zeer veel kristallagen tegelijkertijd gemaakt worden. Hiermee kan dus eenvoudiger een kwalitatief hoogstaand kristal met een groot volume gefabriceerd worden, hetgeen onze motivatie is voor het onderzoeken van dit fabricageconcept.

\section{Dit proefschrift}

In onze groepen aan de Universiteit Twente en het FOM instituut AMOLF worden fotonische kristallen gemaakt om hun optische eigenschappen te kunnen bestuderen. Daarnaast wordt het gedrag van lichtbonnen in het kristal onderzocht en worden de kristallen ultrasnel "geschakeld" waardoor de frequentie van de bandkloof eventjes verandert; een effect dat door veel groepen

\footnotetext{
${ }^{4}$ Door middel van een etsstap worden de poriën van lucht gevormd in de silicium wafers.
} 
wordt onderzocht. Voor dit werk heeft het de voorkeur dat het onderzochte kristal een zo breed mogelijke fotonische bandkloof heeft. In dit proefschrift zijn verschillende fabricagemethoden onderzocht waarmee we geïnverteerde houtstapel kristallen willen maken die hieraan voldoen. Een belangrijk aspect van de onderzochte methoden is dat ze compatibel moeten zijn met hedendaagse technieken die gebruikt worden in de halfgeleider industrie. Als dit het geval is, kunnen de gemaakte fotonische kristallen eenvoudig geïntegreerd worden in bestaande silicium chips. Verder zijn er berekeningen gedaan om het effect van wanorde in de kristallen op de breedte van de bandkloof te bestuderen. Ook wordt een nieuwe methode beschreven waarmee mogelijk trilholtes gemaakt kunnen worden in kunstmatige opalen. Hoofdstuk 1 van het proefschrift is hierboven al samengevat. Daarnaast bevat het proefschrift de volgende hoofdstukken:

\section{Hoofdstuk 2: fabricageschema en berekeningen}

Een geïnverteerde houtstapel kristal kan worden verkregen door twee identieke patronen van poriën van lucht in het silicium te etsen, zie Figuur 4. Deze patronen staan loodrecht op elkaar en zijn zeer nauwkeurig op elkaar uitgelijnd. De bestudeerde fabricagemethodes zijn gebaseerd op twee principes:

- De voorkeursroute is om het eerste patroon van poriën foto-elektrochemisch te etsen en daarna de tweede richting door middel van een Bosch reactief ionen etsproces. Hiervoor is een houderwafer nodig om het monster accuraat uit te kunnen lijnen.

- Snel maken van prototype fotonische kristallen met behulp van een gefocusseerde ionenbundel.

Ondanks dat de bandkloof in geïnverteerde houtstapel kristallen normaal gesproken robuust is tegen wanorde hebben we laten zien dat de bandkloof snel kleiner wordt als de poriën van lucht conisch zijn in plaats van cilindrisch. Onze fabricagemethoden resulteren in lichtelijk conische poriën, maar uit de berekeningen blijkt dat binnen de limieten van onze fabricagemethode de verwachte bandkloof nog steeds breed is. Ook is er een verbetering van de layout van het geïnverteerde bandkloof kristal bestudeerd. Hieruit blijkt dat als de layout lichtelijk gewijzigd wordt, de maximaal te verkrijgen bandkloof toeneemt. Door de gebruikte lithografische processen iets te wijzigen is het mogelijk het kristal volgens deze verbeterde layout te maken. 


\section{Hoofdstuk 3: foto-elektrochemisch etsen van poriën}

Een opstelling is gemaakt voor het foto-elektrochemisch etsen. Deze opstelling is uitvoerig beschreven en getest. De tot nu toe beste etsresultaten zijn gepresenteerd en geanalyseerd. Daarnaast is het proces beschreven waarmee een patroon is gemaakt om de te etsen posities te definiëren. De etsresultaten geven de richting aan voor toekomstige experimenten om diepe poriën van lucht in silicium te krijgen:

- De opstelling is geschikt voor etsexperimenten. Enkele verbeteringen zijn beschreven om de gewenste poriën te maken.

- De experimentele parameters waarmee de poriën geëtst kunnen worden zijn vastgesteld. Deze kunnen gebruikt worden als startpunt voor toekomstige experimenten.

\section{Hoofdstuk 4: reactief ion etsen van poriën}

Patronen van diepe, hoge aspect ratio poriën van lucht zijn gemaakt door middel van Bosch reactief ionen etsen. De porieposities zijn gedefinieerd door een masker van chroom, gepatroneerd met behulp van diep-UV lithografie. De etsparameters zijn bestudeerd en het proces is geoptimaliseerd om de diepe poriën te kunnen etsen. Een bestaande theorie voor brede geulen is verbeterd. Met de verbeterde theorie hebben we laten zien dat de etssnelheid van de poriën is gerelateerd aan hun aspect ratio. Bepalend voor deze relatie is de ionenspreidingshoek in het plasma.

Een vergelijking voor de maximaal haalbare aspect ratio is opgesteld en het blijkt dat het minimaliseren van de ongewenste zijwaartse etscomponent de sleutel is om diepere poriën te maken. Poriën zijn succesvol gemaakt met wereldrecord aspect ratios van meer dan 26. Omdat dit proces veel beter presteert dan verwacht, zien we het als een nieuwe route om beide patronen van poriën te etsen in het geïnverteerde houtstapel kristal.

\section{Hoofdstuk 5: maken en testen van een houderwafer}

Een houderwafer is noodzakelijk in de voorkeurs fabricageroute. Deze houderwafer zorgt ervoor dat de beide patronen van poriën nauwkeurig op elkaar uitgelijnd kunnen worden. Normaal gesproken worden lithografische processen in de silicium chip-industrie enkel in het platte vlak gebruikt. Deze houderwafer is uniek in de zin dat het driedimensionale lithografie mogelijk maakt. Het maken en testen van een geschikte houderwafer is beschreven. Verder is beschreven hoe testmonsters goed uitgelijnd zijn geplaatst ten 
opzichte van het oppervlak in de houderwafer. De testmonsters bleken voldoende goed vastgezet te zijn in de houderwafer. Vervolgens is hierop chroom gedeponeerd en gepatroneerd als etsmasker met behulp van een gefocusseerde ionenbundel. Een voorwaarde voor het nauwkeurig plaatsen van een monster in de houderwafer is dat de doorsnede van het monster vlak is. In dit proefschrift is beschreven hoe een monster met daarin poriën van lucht vlak is gemaakt door te polijsten. Tijdens dit polijsten is de structuur verstevigd met uitgehard fotoresist. Uiteindelijk is gebleken dat de gehele onderzochte methode om monsters in een houderwafer te plaatsen geschikt is om te gebruiken tijdens de fabricage van geïnverteerde houtstapel kristallen.

\section{Hoofdstuk 6: fabricage van potentiële trilholtes in een opaal}

De fabricage van potentiële trilholtes en matrices hiervan in opalen van colloïdale deeltjes is beschreven. Deze holtes zijn gemaakt met behulp van een gefocusseerde ionenbundel. De resultaten demonstreren dat de colloïdale deeltjes volledig doorboord kunnen worden met de gefocusseerde ionenbundel. De straal en de positie van de holte kan met nanometer precisie bepaald worden en de relatie tussen de straal van de holte en de procestijd is vastgesteld.

Met behulp van een optische microscoop is aangetoond dat de holtes interactie vertonen met licht in verschillende richtingen. Deze methode is dus zeer geschikt om trilholtes in geïnverteerde (kunstmatige) opalen te maken. Om dit te bewerkstelligen zullen de gefabriceerde holtes in de toekomst bedekt worden met extra opaallagen en daarna geïnverteerd met silicium of titaniumdioxide. De ontwikkelde methodiek is algemeen inzetbaar als een gefocusseerde ionenbundel gebruikt wordt om niet-geleidende substraten te bewerken.

\section{Hoofdstuk 7: optische reflectiviteitsmetingen}

Optische reflectiviteitsmetingen aan tweedimensionale structuren die gemaakt zijn met Bosch reactief ion etsen laten zien dat dit fotonische kristallen zijn van hoge kwaliteit en een sterke interactie met licht vertonen. Het blijkt dat ingewikkelde pieken ontstaan door de even ingewikkelde bandenstructuren. De meeste pieken in de reflectiveitsspectra kunnen kwantitatief verklaard worden met behulp van berekende bandenstructuren en Braggreflectie.

Met behulp van een gefocusseerde ionenbundel zijn succesvol prototype geïnverteerde houtstapels gemaakt. Berekeningen laten zien dat er in dit kristal in alle richtingen sterke interactie met licht plaatsvindt. Optische 
reflectiviteitsmetingen in één richting laten zien dat deze verwachte sterke interactie inderdaad aanwezig is. Tijdens de bewerkingstechniek met een gefocusseerde ionenbundel slaat het verwijderde silicium opnieuw neer in de structuur. Hierdoor zijn de poriën conisch en enigszins onregelmatig van vorm. De gemaakte structuur is licht wanordelijk. Berekeningen laten zien dat hierdoor een bandkloof net niet aanwezig is.

Het succes van de optische experimenten bevestigt het belang van prototype kristallen, gemaakt met een gefocusseerde ionenbundel. Deze resultaten benadrukken de robuustheid van geïnverteerde houtstapel kristallen tegen wanorde en zijn een extra motivatie voor het maken van geïnverteerde houtstapel kristallen met behulp van de voorskeursfabricageroute.

\section{Hoofdstuk 8: conclusies en perspectief}

In dit proefschrift is de fabricageroute onderzocht waarmee optimale geïnverteerde houtstapel kristallen gemaakt gaan worden. De essentiële fabricagemethoden zijn bestudeerd en de juiste procesparameters geïndentificeerd. Optische reflectiviteitsmetingen laten zien dat de gemaakte structuren, waaronder een prototype geïnverteerd houtstapel kristal, een hoge kwaliteit hebben. Deze resultaten laten zien dat we binnenkort de eerste echte geïnverteerde houtstapel kristallen met een brede bandkloof kunnen verwachten. De resultaten in dit proefschrift beschrijven de laatste stappen die te zetten zijn om dit spannende doel te behalen. Ook zijn potentiële trilholtes van hoge kwaliteit gemaakt in kunstmatige opalen. De gemaakte holtes zullen in de toekomst gebruikt worden om trilholtes in geïnverteerde kunstmatige opalen te verkrijgen. 


\section{Dankwoord}

Het laatste onderdeel van dit proefschrift is het dankwoord. Het mag duidelijk zijn dat de uitdrukking "last but not least" hier van toepassing is. Veel mensen hebben op één of andere wijze bijgedragen aan dit proefschrift. Tijdens koffiepauzes hebben we gekscherend gezegd dat "Iedereen hartstikke bedankt!" zou volstaan om hen te bedanken. Ondanks dat ik iedereen inderdaad hartstikke bedank wil ik hieraan toch graag een persoonlijker tintje geven.

Allereerst wil ik mijn promotor Willem Vos bedanken. Willem, je hebt me enorm veel geleerd over wetenschappelijk onderzoek. Je grenzeloze enthousiasme en je vertrouwen in het kunnen van de mensen in je groep(en) is een constante inspirerende factor. Hiervoor wil ik je bedanken, maar ook vooral voor de kans die je me gegeven hebt om aan dit proefschrift te werken en voor alle hulp die ik daarbij van je ontvangen heb. Het spijt me dat de werkwoorden in mijn Engelse zinnen vaak koppig op de verkeerde plek blijven staan, maar ik weet zeker dat ik dat nog wel eens onder de knie krijg.

Mijn assistent-promotor is Willem Tjerkstra. Willem, je hebt verwoed pogingen gedaan om de voortgang van dit project onder controle te krijgen en te houden. Welnu, het boek is af, dus het is gelukt. Naast de gesprekken over voortgang en de soms ingewikkelde planning, hebben we vele discussies gehad over wetenschap en over technologie: natetsen, droogetsen, houderwafers, enzovoorts. Het is erg fijn om een deskundig klankbord zoals jou te hebben. We waren het niet altijd direct eens, maar werden het (meestal) wel. Voor 
al je begeleiding wil ik je hartelijk danken.

Graag wil ik Ad Lagendijk hier noemen. Ad, ik herinner me nog goed één van de vragen die je me stelde toen ik bij COPS solliciteerde. Deze vraag was erg verrassend en onverwacht, maar vooral to-the-point. Het bleek al snel dat je vragen en opmerkingen meestal van die aard zijn. Je hebt een uitzonderlijk vermogen om mensen op scherp te zetten en ze via andere wegen over vraagstukken na te laten denken. Voor je constructieve bijdragen wil ik je danken en natuurlijk ook voor het feit dat je lid van mijn promotiecommissie bent.

Allard, soms lijkt het wel alsof je alles weet. Ik kan me niet herinneren dat iemand je een vraag stelde en je geen zinnig antwoord had. Het hoeft dan ook niemand te verbazen dat ik vaak bij je heb aangeklopt met vragen over verschillende onderwerpen. Het resultaat van je adviezen is terug te vinden op diverse plaatsen in dit proefschrift. Hiervoor ben ik je erg dankbaar.

De groep Complex Photonic Systems waar ik aan dit boek heb gewerkt bestaat uit een unieke verzameling van bijzondere mensen uit diverse wetenschappelijke disciplines. Hetzelfde geldt voor de "zustergroepen" aan het AMOLF, de Photonic BandGaps groep en de Photon Scattering groep. Het contact met deze mensen in vergaderingen, discussies, koffiepauzes, enzovoorts, maakte het werken aan dit proefschrift ontzettend veel leuker. Hiervoor wil ik hen bedanken.

Van COPS: Bas (wat had ik gemoeten zonder jouw computer-EHBO?), Raymond (ik vond het erg jammer dat ik niet bij je bruiloft kon zijn), Hannie (ik heb bewondering voor de voortvarendheid waarmee je de fabricage van spiegels en kristallen hebt opgepakt), Cock (ook jij bent een bron van kennis die ik veel heb aangesproken. Bedankt voor je altijd aanwezige interesse), Karen Munnink (het spijt me dat je vaak lang moest wachten op administratieve dingen die je me had gevraagd), Elbert (misschien dat ik ooit Metal Gear Solid nog wel eens aanschaf. Aan jouw reclame daarvoor zal het in ieder geval niet liggen), Danang en Rajesh.

Philip "Master" Harding, ook jij hebt me veel geholpen. Denk aan PovRay, ${ }_{\mathrm{AT}} \mathrm{T}$, Linux, de Engelse taal, enzovoorts. Op de valreep heb je mijn Nederlandse Samenvatting gelezen en van advies voorzien. Bedankt voor al deze dingen (overigens zijn je plaatjes niet zo onleesbaar als dat ik je altijd heb voorgehouden). Karen van der Molen wil ik bedanken voor de prettige tijd als kamergenote. Ik vond het enorm gezellig en vind het jammer dat je hier niet meer bent. Veel succes in de consultancy. Ivo, je bent een echte alleskunner. Ik wil je bedanken voor alle keren dat ik je heb aangeschoten met vragen over bijvoorbeeld wetenschap en $\mathrm{AT}_{\mathrm{E} X}$.

Van de photon scattering groep: Sanli, Bernard (ik vond het erg leuk je bij 
de cursus loopbaanplanning tegen te komen), Ramy, Paolo (Paula), Timmo (doet de handschoenenkast het al?), Patrick, Frans, Otto en Bergin.

Van de photonic bandgap groep: Georgios, Merel, Iwert en Edwin (voor mij blijf je toch altijd 'webmaster'). Van deze groep wil ik in het bijzonder Bart en Alex noemen: onze samenwerking heeft tot resultaten geleid die we terug vinden in dit proefschrift. Deze samenwerking heb ik leerzaam en erg leuk gevonden. Bart, ik ben blij dat je mijn paranimf wilt zijn.

Verschillende studenten hebben hun afstudeerprojecten bij COPS, PS of PBG gedaan of zijn daar mee bezig: Wouter, Karin, Frerik (je wint met gemak de prijs voor "best-dressed" student van COPS), Niels, Rob (bedankt voor je hulp bij het maken van dunne opalen), Simon, Adriaan, Oscar, Arie en Peter Zijlstra. Bedankt voor jullie positieve bijdrage aan de sfeer in de groep en aan de verschillende projecten.

Velen hebben de groep(en) ondertussen alweer verlaten. Ook hen wil ik bedanken: Tom (jouw template heeft me geïntroduceerd in de wereld van $\mathrm{IAT}_{\mathrm{E}} \mathrm{X}$ ), Ivan, Martijn, Lydia (van wie ik het maken van fotonische kristallen heb geleerd. We missen je als de sociale motor van COPS), Peter Lodahl (niet vergeten: "Dr. Pepper solves all your problems"), Boris, Tijmen (ik kan niet anders zeggen: jij bent de "Master-Switcher". Ik heb het altijd gewaardeerd dat het bij één permanent geschakeld kristal is gebleven), Dmitry, Femius, Steven, Pedro, Valentin, Charles, Soesila, Vitaly en Rudolf.

Ruud Balkenende, John Kelly, Fred Roozeboom, Irwan Setija en Rutger Voets bedank ik voor inspirerende discussies en hun bijdrage aan het "geïnverteerde houtstapel" project en Meint de Boer en Henri Jansen voor hun hulp en advies bij het reactief ion etsen. Henri en Irwan, ik vind het erg leuk dat onze samenwerking een artikel heeft opgeleverd dat zelfs in het NRC aandacht heeft gekregen.

Veel van het werk in dit proefschrift zou onmogelijk zijn geweest zonder een goed werkende cleanroom. Ik wil dan ook de volgende MESA+ cleanroom stafleden hartelijk bedanken: Gerard, Samantha, Eddy, Rene, Huib, Marion, Hans, Peter en Ite-Jan. Bert Otter en Vishwas Gadgil ben ik dankbaar voor hun hulp met de gefocusseerde ionenbundel en van het CMA laboratorium Rico Keim en Mark Smithers voor hun hulp bij de monsterbewerking en karakterisatie. Mark en Vishwas wil ik ook nog bedanken dat ze het hebben aangedurfd hun apparatuur (FIB en SEM) aan mij toe te vertrouwen en voor de uitleg die ze me hebben gegeven om deze apparatuur te kunnen bedienen.

COPS mag zich gelukkig prijzen met de gezellige buren van TCO. Daarnaast hebben Klaas, Rob, André, Theo, Jan en George ons regelmatig geholpen met onze vacuümspullen of door een gadget te maken. Hiervoor wil ik jullie bedanken. Rindert van TCO E\&A bedank ik voor het schrijven 
van het Labview programma.

Ook wil ik de mensen van de Universiteitsbibliotheek, Marijke Broekhuis en Wim Oosterling in het bijzonder, bedanken voor alle hulp bij het zoeken naar boeken en artikelen. Het secretariaat en receptie van het AMOLF wil ik bedanken voor het doorgeven van de vele dikke faxen die ik aan Willem Vos stuurde en van hem ontving op de dagen dat hij in Amsterdam was.

De aanwezigheid van familie en vrienden wordt te vaak als vanzelfsprekend aangenomen. Helaas ben ik daar ook regelmatig schuldig aan. Ik wil hier dan ook benadrukken dat ik jullie steun enorm waardeer, bedankt! $\mathrm{Pa}$, ontzettend bedankt voor meer dan 30 jaar ouderlijke hulp en interesse. Laurens, ik stel het erg op prijs dat je mijn paranimf wil zijn tijdens de promotie. Wat kan er mis gaan met mijn grote broer naast me?

Hiermee kom ik aan het eind van dit dankwoord. Mocht ik iemand vergeten zijn, dan bied ik daarvoor mijn welgemeende excuses aan. De laatste personen die ik bedank zijn de belangrijkste in mijn leven: lieve Eline, Cas en Engelien, ik ben ontzettend blij dat jullie elke dag bij me zijn. De laatste paar maanden heb ik veel tijd gestoken in dit proefschrift. Niets doet me meer plezier dan dit de komende tijd goed te gaan maken.

Léon A. Woldering.

Augustus 2008 





